\title{
Die Wahrnehmung des Bildungsangebots durch arme Haushalte in Entwicklungsländern
}

\author{
Das Fallbeispiel Lima, Peru
}

\author{
DISSERTATION \\ zur Erlangung des wirtschaftswissenschaftlichen Doktorgrades \\ der Wirtschaftswissenschaftlichen Fakultät \\ der Universität Göttingen
}

vorgelegt von

Anke Scholz

aus Großburgwedel

Göttingen, 2003 



\section{INHALTSVERZEICHNIS}

0. Einleitung

\section{TEIL: THEORETISCHE GRUNDLAGEN}

1. Entstehung und Wirkungsbereich der Bildungsökonomie........ 15

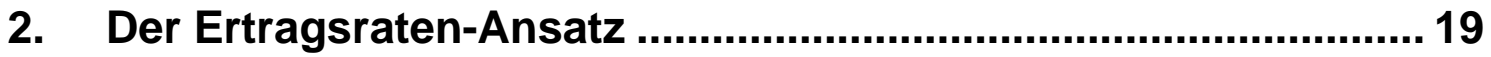

$2.1 \quad$ Darstellung des Ansatzes......................................................... 19

2.1.1 Bildung als Investition oder Konsum .............................. 19

2.1.2 Kosten und Nutzen von Bildungsinvestitionen ................. 21

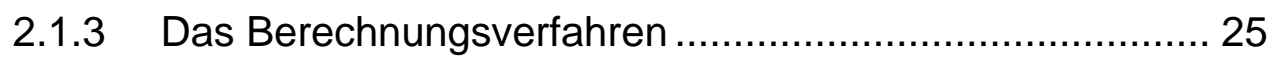

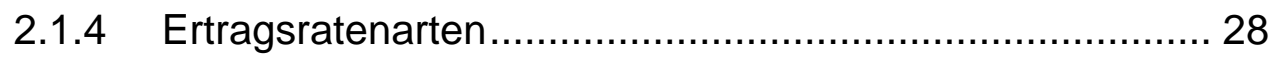

2.2 Kritik am Konzept der Ertragsraten ......................................... 31

2.3 Einige empirische Ergebnisse von Ertragsratenberechnungen ..... 34

2.4 Fazit und Anwendbarkeit des Ertragsratenansatzes .................... 39

3. Die optimale Bildungsentscheidung …....................................... 41

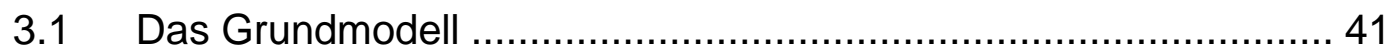

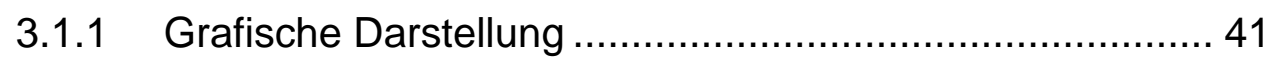

3.1.2 Die Lage der Kurven..................................................... 43

3.2 Individuelle Kurvenverläufe ..................................................... 45

3.3 Fazit zur Anwendung der grafischen Darstellung ....................... 47

4. Die Grenzen der ökonomischen Modelle ................................... 49

4.1 Der Erwartungsbildungsprozess................................................. 49

4.2 Der Prozess der Bildungsentscheidung .................................... 52

4.3 Auswirkungen auf die Modellanwendung ................................... 58 


\section{TEIL: AUSWERTUNG EMPIRISCHER STUDIEN ZU DEN DETERMINANTEN VON BILDUNGSINVESTITIONEN}

5. Schätzungen der Bildungsnachfrage? ....................................59

6. Kosten- und Nutzenfaktoren als Bildungsdeterminanten ..........65

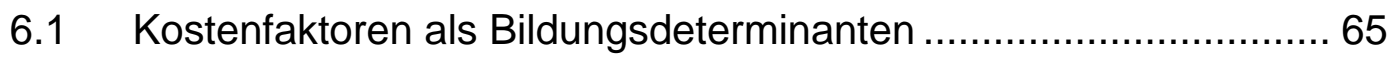

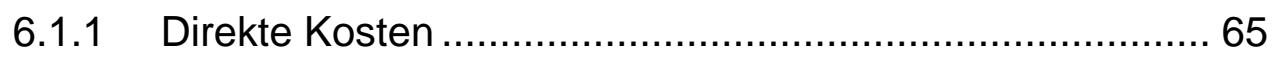

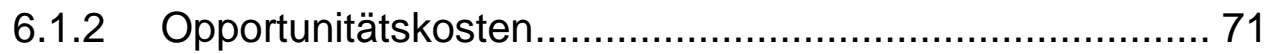

6.1.2.1 Entgangene Einkommen ......................................... 72

6.1.2.2 Fehlende Mithilfe im Haushalt ................................... 75

6.1.2.3 Zusammenfassung der Opportunitätskosten............. 83

6.2 Nutzenfaktoren als Bildungsdeterminante .................................. 85

6.2.1 Erwartete Nutzen der Ausbildung .................................. 85

6.2.2 Erwartete Bildungserträge - Allgemeine Erkenntnisse......... 87

6.3 Kapitalmarktrestriktionen als Bildungsdeterminante ..................... 91

6.4 Zusammenfassung der Nutzen- und Kostenfaktoren .................... 94

7. Familiäre und individuelle Merkmale als Bildungsdeterminanten

7.1 Haushaltscharakteristika als Bildungsdeterminanten ................... 97

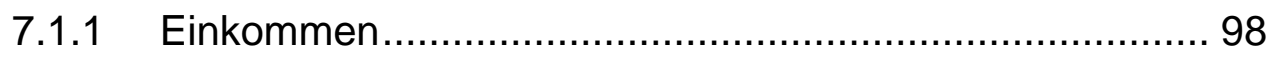

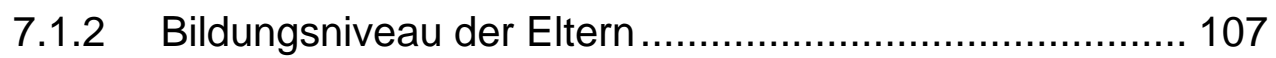

7.1.3 Geschwisteranzahl .................................................... 114

7.2 Charakteristika der Kinder als Bildungsdeterminanten ............... 119

7.2.1 Alter der Kinder...................................................... 119

7.2.2 Geschlecht der Kinder ................................................ 120

7.3 Zusammenfassung der familiären und individuellen Merkmale ... 123

8. Zusammenfassung der Erkenntnisse empirischer Studien.... 125 


\section{TEIL: AUSWERTUNG DER HAUSHALTSBEFRAGUNG}

9. Grundlagen zur eigenen Haushaltsbefragung ….................... 127

9.1 Das peruanische Bildungssystem ...................................... 127

9.2 Die Bevölkerungsstruktur Limas und die Stichprobenauswahl .... 131

10. Beschreibung der sozioökonomischen Schichten .................. 133

10.1 Sozioökonomische Charakterisierung der Schichten ................. 133

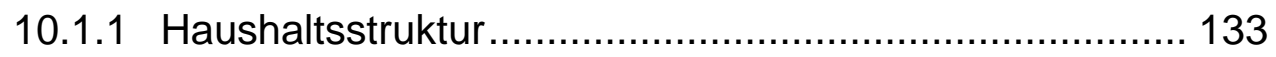

10.1.2 Herkunft der Familie .................................................... 136

10.1.3 Ausbildungssituation der Familien ............................... 139

10.1.4 Ökonomische Situation des Haushaltes ........................ 141

10.1.5 Zusammenfassung der Charakterisierung..................... 144

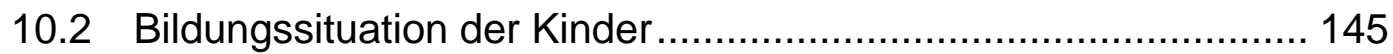

10.2.1 Der typische Bildungsweg ........................................ 145

10.2.2 Abbrecherverhalten während der Basisausbildung......... 148

10.2.3 Leistungen in der Basisausbildung ............................. 150

10.2.4 Nebentätigkeiten der Schüler..................................... 154

10.2.5 Zusammenfassung der Bildungssituation ...................... 156

11. Bildungswunsch und Einkommenserwartungen der Eltern ... 157

11.1 Gewünschtes Bildungsniveau für die Kinder ............................ 157

11.2 Einkommenserwartungen am Arbeitsmarkt.............................. 159

11.3 Das Rentabilitätseinkommen für die eigenen Kinder ................... 161

11.4 Erwartungen über die Rentabilität der Ausbildung ..................... 164

11.4.1 Gemeinsamkeiten in den Rentabilitätserwartungen........ 164

11.4.1.1 Gesamtrentabilität der Bildungsstufen ...................... 164

11.4.1.2 Grenzbetrachtung der Rentabilitätserwartungen ..... 167

11.4.1.3 Zusammenfassung und Schlussfolgerungen........... 169

11.4.2 Unterschiede in den Ertragserwartungen ..................... 171

11.4.2.1 Einkommenserwartungen am Arbeitsmarkt............. 171

11.4.2.2 Einkommenserwartungen für die eigenen Kinder.... 173

11.4.2.3 Ansprüche an die Rentabilitätseinkommen ............. 175

11.4.2.4 Gesamtrentabilität der Bildungsstufen..................... 177

11.4.2.5 Grenzbetrachtung der Rentabilitätserwartungen ..... 178

11.4.2.6 Zusammenfassung und Schlussfolgerungen........... 179

11.5 Zusammenfassung der Wünsche und Erwartungen.................... 181 
12. Sonstige Einstellungen gegenüber der Ausbildung 183

12.1 Einstellung zu einer Beschäftigung der Kinder .......................... 183

12.2 Bewertung der Abwesenheit der Kinder .................................. 185

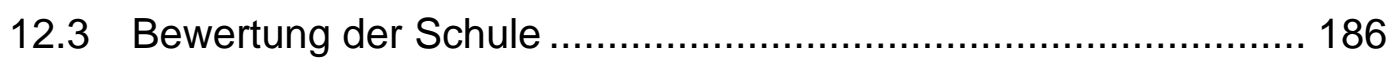

12.4 Zusammenfassung der sonstigen Einstellungen ....................... 189

\section{TEIL: ZUSAMMENFASSUNG UND SCHLUSSFOLGERUNGEN}

13. Zusammenfassung und grafische Darstellung des privaten Investitionskalküls

14. Auswirkungen von schichtenspezifischen Unterschieden in den Ertragserwartungen für höhere Bildung ................................. 197

14.1 Unter der Annahme vergleichbarer Schulen............................. 198

14.1.1 Typische Finanzierungskostenkurven........................... 199

14.1.2 Typische Ertragsratenverläufe .................................... 202

14.1.3 Optimale Bildungsniveaus ............................................ 204

14.2 Unter Einbeziehung von privaten Schulen................................ 206

14.2.1 Auswirkungen auf die Ertragsratenkurven ..................... 206

14.2.2 Auswirkungen auf die Finanzierungskostenkurven......... 208

14.2.3 Auswirkungen auf die optimalen Bildungsniveaus .......... 209

14.3 Zusammenfassung der Ergebnisse und Schlussfolgerungen...... 213

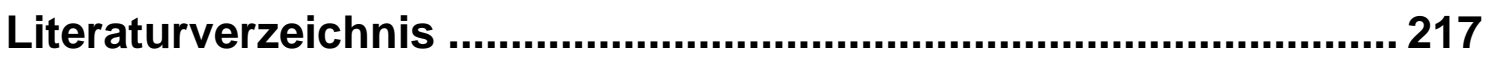

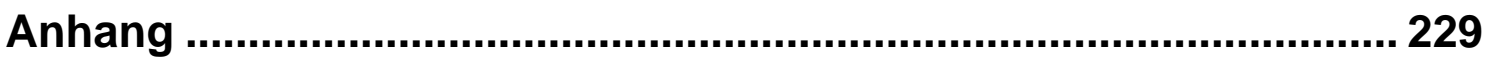




\section{Verzeichnis der Abbildungen}

Abbildung 2.1: Bildung als Investitions- und Konsumgut 20

Abbildung 2.2: Interne Effekte von Bildungsinvestitionen ............................ 22

Abbildung 2.3: Externe Effekte von Bildungsinvestitionen .............................. 23

\section{Verzeichnis der Grafiken}

Grafik 3.1: Die optimale individuelle Investitionsentscheidung.................... 42

Grafik 3.2: Variierende Ertragsraten und Finanzbedingungen .................... 45

Grafik 13.1: Kapitalkosten und Ertragsraten für die Basisausbildung ........... 193

Grafik 13.2: Verlaufsänderung der Ertragsraten für die höhere Bildung ....... 195

Grafik 13.3: Kurvenverläufe für die höhere Bildung ................................... 196

Grafik 14.1: Individuelle Finanzierungskostenkurve.................................. 199

Grafik 14.2: Finanzierungskostenkurve für arme und reiche Haushalte ....... 202

Grafik 14.3: Typische Ertragsratenkurven für arme und reiche Haushalte ... 204

Grafik 14.4: Optimale Bildungsniveaus für arme und reiche Haushalte....... 205

Grafik 14.5: Ertragsraten bei alternativen Schultypen................................ 207

Grafik 14.6: Finanzierungskosten bei alternativen Schultypen .................... 208

Grafik 14.7: Mögliche Verläufe der Finanzierungskostenkurve..................... 210

\section{Verzeichnis der Tabellen}

Tabelle 2.1: Ertragsraten von Bildungsinvestitionen, reg. Durchschnitt ........ 34

Tabelle 2.2: Soziale Ertragsraten in Kenia, 1980 ...................................... 37

Tabelle 6.1: Schulgebühren als Bildungsdeterminante …............................. 66

Tabelle 6.2: Entfernung als Bildungsdeterminante ……............................ 68

Tabelle 6.3: Entgangene Einkommen als Bildungsdeterminante ................. 73

Tabelle 6.4: Tätigkeit des Haushaltsvorstandes als Bildungsdeterminante .. 76

Tabelle 6.5: Alter und Geschlecht des Kindes als Bildungsdeterminante .... 79

Tabelle 6.6: Familienstruktur als Bildungsdeterminante............................. 80

Tabelle 6.7: Besitztümer als Bildungsdeterminante .................................... 82

Tabelle 7.1: Familieneinkommen als Bildungsdeterminante ........................ 99

Tabelle 7.2: Konsumausgaben als Bildungsdeterminante ......................... 102

Tabelle 7.3: Besitz von Wertgegenständen als Bildungsdeterminante ....... 106

Tabelle 7.4: Bildungsniveau der Eltern als Bildungsdeterminante .............. 108 
Tabelle 7.5: Geschwisteranzahl als Bildungsdeterminante 115

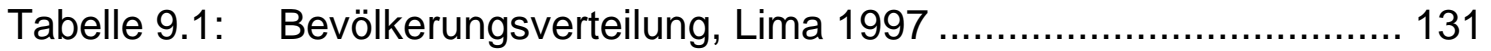

Tabelle 9.2: Haushalte mit Kindern im schulfähigen Alter, Lima 1997. 132

Tabelle 10.1: Familienstruktur der Stichprobe 134

Tabelle 10.2: Charakteristika der Haushaltsvorstände ............................... 135

Tabelle 10.3: Wer trifft die Bildungsentscheidung................................. 135

Tabelle 10.4: Muttersprache des Hauhaltsvorstandes........................... 136

Tabelle 10.5: Migrationsverhalten der Eltern ..................................... 137

Tabelle 10.6: Anwesenheit in Lima, Jahre ........................................... 137

Tabelle 10.7: Motive für die Migration nach Lima ................................. 138

Tabelle 10.8: Schulbildung der Eltern ............................................ 139

Tabelle 10.9: Abbruch der weiterführenden Schule nach max. 3 Jahren...... 139

Tabelle 10.10: Abgeschlossene Schuljahre der Eltern ............................ 140

Tabelle 10.11: Besuch einer höheren Schule ...................................... 140

Tabelle 10.12: Leseverhalten der Eltern ........................................... 141

Tabelle 10.13: Beschäftigungssituation der Eltern ............................... 142

Tabelle 10.14: Durchschnittliches monatliches Einkommen ........................ 142

Tabelle 10.15: Wohnverhältnisse der Familien ........................................ 143

Tabelle 10.16: Geräteausstattung der Wohnungen ............................... 143

Tabelle 10.17: Sozioökonomische Charakteristika der Haushalte ................. 144

Tabelle 10.18: Besuch eines Kindergartens ......................................... 145

Tabelle 10.19: Art der besuchten Schule ............................................ 146

Tabelle 10.20: Transportmittel zur Schule ........................................... 146

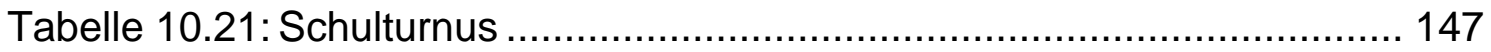

Tabelle 10.22: Unterstützung des Schulbesuchs durch die Eltern................ 148

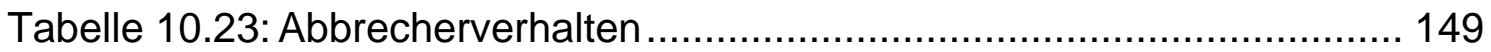

Tabelle 10.24: Gründe für das Verlassen der Schule ............................... 149

Tabelle 10.25: Besuch der Bildungsstufen nach dem Alter, Primarschule...... 150

Tabelle 10.26: Besuch der Bildungsstufen nach dem Alter, Sekundaria ........ 151

Tabelle 10.27: Bildungsstufen nach ausgewählten Altersgruppen................. 151

Tabelle 10.28: Wiederholerverhalten der Schüler..................................... 152

Tabelle 10.29: Wiederholungen nach Bildungsstufen ............................ 152

Tabelle 10.30: Wiederholungen in der Primaria.................................... 153

Tabelle 10.31: Beurteilungen der Schüler durch die eigenen Eltern .............. 153

Tabelle 10.32: Ausübung einer bezahlten Beschäftigung .......................... 154 


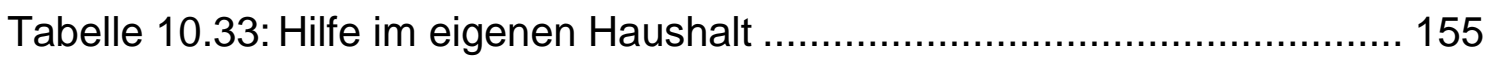

Tabelle 10.34: Charakteristika der Basisausbildung der Kinder..................... 156

Tabelle 11.1: Gewünschtes Bildungsniveau für die eigenen Kinder ............. 157

Tabelle 11.2: Erwartete Einkommensdifferenzen, Soles ............................ 159

Tabelle 11.3: Erwartete Einkommensdifferenzen, \% ................................. 159

Tabelle 11.4: Einkommens- und Rentabilitätserwartungen, eigene Kinder... 164

Tabelle 11.5: Erwartete Einkommen als \% der Rentabilitätseinkommen ...... 165

Tabelle 11.6: Rentabilitätserwartungen der Ausbildung, eigene Kinder........ 165

Tabelle 11.7: Reicht die Basisausbildung aus?? ........................................ 166

Tabelle 11.8: Einkommensänderungen durch den Besuch der Sekundaria . 167

Tabelle 11.9: Anstiegsraten mit dem Bildungsniveau ................................. 168

Tabelle 11.10: Erwartete Einkommen nach Bildungsniveaus ........................ 172

Tabelle 11.11: Erwartete Einkommen nach Schichten ................................ 174

Tabelle 11.12: Diskriminierung der eigenen Kinder am Arbeitsmarkt ............. 174

Tabelle 11.13: Erwartete Rentabilitätseinkommen nach Schichten ................ 176

Tabelle 12.1: Bezahlte Arbeit anstelle des Schulbesuchs............................. 183

Tabelle 12.2: Bezahlte Arbeit neben dem Schulbesuchs............................ 184

Tabelle 12.3: Empfindungen bei Abwesenheit der Kinder ............................ 185

Tabelle 12.4: Wertschätzung der Lehrer in der Gesellschaft ....................... 186

Tabelle 12.5: Auswirkungen der Ausbildung auf die Kinder......................... 187

Tabelle 12.6: Aufgaben der Schule...................................................... 188

Tabelle A 1: Dominierende Merkmale der Schichten .................................. 229

Tabelle A 2: Bevölkerungsverteilung: Lima Stadt.................................... 230

Tabelle A 3: Bevölkerungsverteilung: Lima Stadt, \%............................... 231

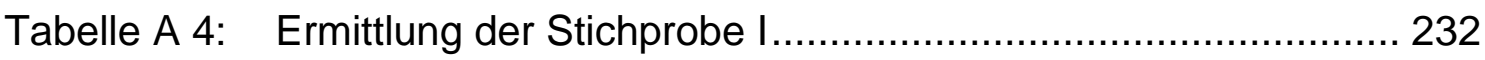

Tabelle A 5: Ermittlung der Stichprobe II............................................. 233

Tabelle A 6: Lima: Verteilung der Bevölkerung auf Haushalte, 1994 .......... 234

Tabelle A 7: Lima: Haushalte, Kindern im schulpflichtigen Alter, 1997 ....... 234

Tabelle A 8: Lima: Expansionsfaktoren, 1997 ....................................... 234

Tabelle A 9: Varianzanalyse, Ertragserwartungen allgemein ..................... 235

Tabelle A 10: Varianzanalyse, Ertragserwartungen, eigene Kinder .............. 236 



\section{Einleitung}

Schon auf der Weltbildungskonferenz in Jomtien 1990 wurde das Ziel „Bildung für Alle" deklariert. Bis zum Jahre 2000 sollte es keine Analphabeten mehr geben. Auf der Weltbildungskonferenz 2000 in Dakar wurde das Resumee gezogen: Ziel nicht erreicht. Angesichts der ernüchternden Bilanz erneuerte das Dakar-Forum das Ziel der Grundbildung für Alle nun mit neuer Jahreszahl. Ein internationaler Aktionsplan verpflichtet die Regierungen, bis 2015 die Zahl der Analphabeten weltweit zu halbieren. ${ }^{1}$ Die Staats- und Regierungschefs der G8 beauftragten auf ihrem Gipfel in Genua 2001 eine Educational Task Force mit der Bearbeitung eines Berichts zu der Frage, wie Education for All gefördert werden könne. Parallel dazu entstand der Aktionsplan der Weltbank zur Förderung umfassender Grundbildung in Entwicklungsländern. ${ }^{2}$ Beide Ansätze ergänzen sich zur nachhaltigen Umsetzung der auch in der Millenniums-Erklärung der Vereinten Nationen aufgenommen Ziele - umfassende Grundbildung und gleiche Bildungschancen für Jungen und Mädchen - die zu Kernaufgaben der internationalen Armutsbekämpfung wurden. Hintergrund dieser Entwicklung ist die unstrittige Erkenntnis, dass ohne Grundbildung Entwicklungsprogramme in allen Sektoren an ihre Grenzen stoßen.

Bildungsexperten sind sich einig, dass eine weiterführende Kompetenzentwicklung mindestens fünf bis sechs Jahre Grundbildung voraussetzt. In vielen Entwicklungsländern kann das öffentliche Schulsystem dies nicht gewährleisten, geschweige denn eine angemessenen Weiterbildung. Private Schulträger haben daher zunehmend an Bedeutung gewonnen, was oftmals notwendig und dankenswert, aber sozial nicht unbedenklich ist. Je knapper und teurer der Zugang zu Bildung ist, desto eher werden sozial benachteiligte Gruppen ausgeschlossen. Der Ausschluss ganzer Bevölkerungsgruppen bereits bei der Grundbildung ist in vielen Ländern eine Realität, die angesichts der globalen Entwicklungsziele bis 2015 nicht hingenommen werden kann. ${ }^{3}$

In Peru gilt das quantitative Ziel, ausreichend Primarschulplätze für die Bevölkerung im grundschulfähigen Alter bereitzustellen, als erreicht. ${ }^{4}$ Nun spielt u. a. die Mobilisierung der Nachfrage und die Schaffung komplementärer Bedingungen in den Haushalten eine große Rolle, um Bildungserfolge in allen Bevölke-

Vgl. Baaden (2002), S. 246-248.

Vgl. Wieczorek-Zeul, (2002), S. 236-239.

Vgl. Hofmann/Lock (2002), S. 244-245.

Im März 1997 wurde der Ausbau der Infrastruktur der Schulen für abgeschlossen erklärt. Die physischen Voraussetzungen im Erziehungswesen seien jetzt vorhanden, so der offizielle Tenor. Vgl. dazu KAS Kurzinfo (1997). 
rungsschichten zu gewährleisten. In der vorliegenden Arbeit sollen daher die Verhaltensweisen verschiedener Bevölkerungsgruppen bezüglich ihres Bildungsverhaltens untersucht werden. Dazu wird eine Stichprobe der limenser Bevölkerung herangezogen. Ziel der Untersuchung ist es, nach Gemeinsamkeiten und Unterschieden in der Wahrnehmung der Schulausbildung zwischen Haushalten aus unterschiedlichen sozioökonomischen Bevölkerungsschichten zu forschen.

Die Arbeit verfolgt einen eklektischen Ansatz, um die Erkenntnisse aus unterschiedlichen Quellen zusammen zu bringen. Auf diese Weise soll die mittlerweile unüberschaubare Menge an Veröffentlichungen strukturiert werden. Der Aufbau der Arbeit wird im folgenden etwas genauer dargestellt.

Zunächst wird im ersten Teil der theoretische Rahmen der Bildungsökonomie vorgestellt. Trotz der Kritik an diesem Ansatz und seiner Grenzen bietet er einige Elemente, die zur systematischen Analyse des Entscheidungsprozesses unerlässlich sind. Um die notwendigen Elemente herauszugreifen, wird zunächst ihr Platz innerhalb der Bildungsökonomie erläutert. Damit sie als Instrumente zur Analyse der Ergebnisse einer Haushaltsbefragung benutzt werden können, werden in den ersten vier Kapiteln ihre Stärken und Schwächen dargestellt.

Der zweite Teil zeigt dann, wie bisher mit der Fragestellung nach den Determinanten der Bildungsnachfrage in der empirischen Forschung umgegangen wurde. Die ausführliche Auswertung, Darstellung und Zusammenfassung der Forschungsergebnisse für Entwicklungsländer war notwendig, um aus der umfassenden Literatur zu diesem Thema den Forschungsstand zu ermitteln. Dieser Teil gibt einerseits wichtige Hinweise auf den Entscheidungsprozess, zeigt andererseits aber auch große Lücken in der Erklärung der Bildungsnachfrage auf.

Um einige dieser Lücken zu schließen, wurde eine Haushaltsbefragung in Lima durchgeführt. Ihre Ergebnisse werden im dritten Teil ausführlich dargestellt. Da es in dieser Untersuchung vor allem darum geht, das Bildungsverhalten von unterschiedlichen Bevölkerungsschichten zu vergleichen, werden die Schichten zunächst nach sozioökonomischen Kriterien und der Ausbildung der Kinder charakterisiert. Den Kernpunkt dieses Teils bildet die Auswertung der Befragungsergebnisse zu subjektiven Meinungen und Erwartungen der Eltern bezüglich des Bildungssystems. Vor allem die Erwartungen zu den durch die Bildung induzierten Einkommensanstiegen sind besonders relevant für die Erklärung des Bildungsverhaltens. Damit können zum einen die aus der Theorie bekannten Verhaltensannahmen hinterfragt werden, und zum anderen können Gründe für unterschiedliche Bildungsverhalten bzw. Bildungserfolge aufgedeckt werden. 
Die zum Teil unerwarteten Ergebnisse lassen interessante Schlüsse für die Bildungstheorie und -politik zu.

Den Abschluss der Arbeit bildet dann der vierte Teil, in dem die Ergebnisse der vorherigen drei Teile zusammengebracht werden. Es ergeben sich interessante Schlussfolgerungen für die Bildungsnachfrage unterschiedlicher Bevölkerungsschichten und damit auch für die Bildungspolitik. 



\section{Teil: Theoretische Grundlagen}

\section{Entstehung und Wirkungsbereich der Bildungsökonomie}

Die Bildungsökonomie behandelt die Wechselwirkungen zwischen ökonomischen Prozessen und der Bildung von menschlichen Ressourcen. Unter menschlichen Ressourcen wird dabei die Bevölkerung und deren Ausbildungsstand verstanden.

Obwohl schon Ökonomen wie ADAM SMITH (1776) die Bevölkerung einer Volkswirtschaft zu deren „Kapital“ rechnete, entstand der Begriff „Humankapital“ erst viel später. Im Dezember 1960 hielt THEOdORE W. SCHULTZ einen Vortrag beim jährlichen Treffen der American Economic Association. Die Arbeit wurde im März des folgenden Jahres unter dem Titel 'Investment in Human Capital' im American Economic Review veröffentlicht. ${ }^{5}$ Schultz legte damit den Grundstein für die heutige Bildungsökonomie als einem eigenständigen Forschungsgebiet im Rahmen der Wirtschaftswissenschaften. ${ }^{6}$ Ökonomen wie ScHULTz (1961), BECKER (1975), und MINCER (1974) entwickelten den heute in der Bildungsökonomie vorherrschenden Humankapitalansatz ${ }^{7}$ und beeinflussten die Denkweise von Ökonomen und Sozialwissenschaftlern im Hinblick auf das Bildungswesen. Mittlerweile wurde die Bildungsökonomie in viele Teildisziplinen der Ökonomie integriert.

Auf makroökonomischer Ebene wurde untersucht, welchen Einfluss die Ausbildung auf das Sozialprodukt haben kann. Seit Beginn der 60er Jahre wurde der Faktor Humankapital in die Wachstumsmodelle bzw. makroökonomische Produktionsfunktionen eingebaut. Pionierarbeit bezüglich des GrowthAccounting Ansatzes leistete Denison (1967). ${ }^{8}$

Erst seit Ende der 80er Jahre entwickelte sich die Neue Wachstumstheorie, die die Faktoren Humankapital sowie Forschung und Entwicklung endogenisierte. Auf diesem Gebiet leisteten Romer (1986) und Lucas (1988). Pionierarbeit. ${ }^{9}$ Weiterhin wurde die Mittelverwendung und ihre Auswirkungen auf Effizienz, Ef-

Vgl. Schultz (1961), S. 1-17.

Vgl. Biondi Shaw (1997), S. 15.

Vgl. Woodhall (1987), S. 21-24.

$8 \quad$ Zitiert nach Hicks (1987), S. S. 101-107. Eine kurze Darstellung des Ansatzes findet sich bei Psacharopoulos (1973), S. 111-123. Psacharopoulos (1984) berechnete den Beitrag der Bildung zum Wirtschaftswachstum für 29 Länder, vgl. die Tabelle auf S. 337.

9 Ein Überblick über die Ansätze gibt Arnold (1995), S. 409-445. Vgl. auch Barro (1991) und Mankiw/Romer/Weil (1992), zietiert nach Glewwe (2002), S. 436. Vgl. auch Gould/Ruffin (1995), S. 425445. Timmermann (1994), disaggregierte die Wachstumsdeterminante Bildung in die Bereiche „elementare Bildung“, „höhere Bildung“, „inländische F\&E“ und „Technologietransfer aus dem Ausland“ und schätzte die Wirkungen für eine Gruppe ärmerer und eine Gruppe reicherer Länder. 
fektivität und Verteilung untersucht. Die Erkenntnis, dass ein höheres Ausbildungsniveau das Wirtschaftswachstum und die Entwicklung eines Landes fördern kann ${ }^{10}$, hat die Bildungsökonomie in die Entwicklungsökonomie getragen. Ein weiterer Zweig der Makroökonomie entwickelte soziale Ertragsraten und Finanzierungsmodelle, wobei vor allem das Ziel verfolgt wurde, Anhaltspunkte für eine effiziente Verwendung öffentlicher Ausgaben zu gewinnen. Außerdem versuchte man, die zukünftige Bedarfsstruktur des Arbeitsmarktes zu ermitteln und die Ausbildung daran anzupassen.

Im Bereich der Mikroökonomie wurden vor allem die individuellen Bildungsentscheidungen untersucht. Dieser Frage haben sich sowohl Ökonomen als auch Soziologen angenommen. ${ }^{11}$ Vor allem die Ökonomen unterstellen den Individuen dabei die rationale Abwägung von Nutzen und Kosten der Ausbildung. Seit der Veröffentlichung des "Coleman Report" (1966), der soziokulturellen Faktoren eine wichtigere Rolle bei der Erklärung von Lernergebnissen zuwies als den Schulvariablen ${ }^{12}$, wurden bis 1987 in mehr als 144 Untersuchungen (vor allem für die USA) anhand von Produktionsfunktionen der Zusammenhang zwischen Inputfaktoren und dem Schulerfolg untersucht. ${ }^{13}$ Als sich auch in Entwicklungsländern die Bildungssituation gemessen an traditionellen Indikatoren, wie bspw. die Einschulungsquoten, verbessert hatte, wurde eine große Lücke zwischen eingesetztem Kapital und Bildungsoutput (Leistungsniveau der Schüler) festgestellt. ${ }^{14}$ Seitdem befassen sich viele Studien mit dem Zusammenhang zwischen Schulcharakteristika und den Bildungserfolgen der Schüler, um die interne Effizienz der Bildungszentren zu steigern. ${ }^{15}$

Im Bereich der Entwicklungsökonomie wird die Ausbildung der Bevölkerung mittlerweile als Schlüsselfaktor für den Entwicklungsprozess betrachtet. ${ }^{16}$ Die Erhöhung der Arbeitsproduktivität wird als wichtigste entwicklungsökonomische Folge einer Verbesserung des Bildungsstandes betrachtet. Aber auch die Auswirkungen der Ausbildung auf die Geburten- und Sterberaten, das Migrationsverhalten und den Gesundheitszustand werden hier untersucht. ${ }^{17}$ Durch eine

10 Vgl. dazu z.B. Blaug (1991), Psacharopoulos (1987), Becker (1983), Tilak (1993).

11 Vgl. dazu die Darstellungen in Kapitel 5.

12 Vgl. Psacharopoulos/Woodhall (1985), S. 215.

13 Eine Zusammenfassung der ernüchternden Ergebnisse bezüglich der Schulvariablen liefert Hanushek (1987).

14 Zu Lateinamerika vgl. Schiefelbein (1992), S. 15-26 und Wolff/Schiefelbein/Valenzuela (1994), S. 102.

15 Eine Zusammenfassung von fast 100 quantitativen Studien über die Effektivität von Schulressourcen in Entwicklungsländern bis 1991 gibt Hanushek (1995), S. 230. Vgl auch Lockheed/ Verspoor (1991), Harbison/Hanushek (1992), Haveman/Wolfe (1995) und Glewwe (2002), S. 437.

16 Vgl. DSE (2002). Die Zeitschrift enthält mehrere Artikel, die die neu entdeckte Relevanz der Ausbildung belegen und die Geschichte der Bildungspolitik in der internationalen und der bilateralen Zusammenarbeit aufzeigen.

17 Vgl. z.B. Glewwe (2002), S. 472-479. 
verbesserte Ausbildung soll einer breiteren Schicht der Bevölkerung die Partizipation an ökonomischen und gesellschaftlichen Prozessen ermöglicht werden, um damit das Produktionspotential besser ausnutzen und die Einkommensverteilung verbessern zu können. ${ }^{18}$ Außerdem wird die Fähigkeit, auf den technischen Wandel und die voranschreitende Globalisierung zu reagieren, positiv beeinflusst. Die Erhöhung des Humankapitals armer Bevölkerungsgruppen kann daher als nahezu konfliktfreie Maßnahme betrachtet werden, da sie zugleich allokative und distributive Ziele verfolgen kann. ${ }^{19}$

Für alle diese Bereich wurden Modelle entwickelt und zahlreiche theoretische und empirische Studien unternommen. Eine Darstellung der umfangreichen Literatur zur Bildungsökonomie führt hier zu weit. Die angegebenen Literaturhinweise stellen nur eine begrenzte Auswahl dar, die sich als Ausgangspunkt für weitere Recherche eignet.

Für die vorliegende Arbeit sind genauere Kenntnisse über die Einflussfaktoren der Bildungsnachfrage von großer Bedeutung. In der ökonomischen Theorie wird die Bildungsnachfrage aus einem individuellen Optimierungskalkül abgeleitet, in dem die Ertragsraten eine zentrale Rolle spielen. Daher wird im folgenden die Logik der Ertragsratenermittlung gezeigt, von der angenommen wird, dass sie die Individuen - zumindest in einer groben, intuitiven Form - vornehmen. Mit Hilfe der Ertragsraten kann das Optimierungskalkül dann grafisch dargestellt werden. Den Ausgangspunkt der Arbeit bietet daher die Darstellung des Ertragsratenansatzes und die grafische Darstellung des Optimierungskalküls. Außerdem wird auf die grundsätzliche Problematik der ökonomischen Verhaltenstheorie (rational choice) hingewiesen.

18 Tilak hat den Zusammenhang zwischen Armut und Bildungsinvestitionen auf makroökonomischem Niveau empirisch untersucht und ermittelte eine signifikant negative Korrelation zwischen dem Bildungsstand und dem Anteil der Menschen unterhalb der Armutsgrenze. Vgl. Tilak (1994), S. 66-67. 



\section{Der Ertragsraten-Ansatz}

Das Konzept der Ertragsraten bestimmt bis heute die Diskussion um die Bildungsinvestitionen. Dieses Konzept behandelt Ausbildung als eine Form von Investition in den Menschen, die das Lebenseinkommen der ausgebildeten Individuen und den Nutzen der Gesellschaft als ganzes erhöht. Nutzen-KostenAnalysen wurden herangezogen, um den Wert von Bildungsinvestitionen zu ermitteln. ${ }^{20}$ Aus den Nutzen und den Kosten der Ausbildung wird dabei eine Ertragsrate ermittelt, die den Wert der Bildungsinvestition bestimmt.

\subsection{Darstellung des Ansatzes}

Zunächst soll aufgezeigt werden, was die Ausbildung bewirken kann und welche Schwierigkeiten die Bewertung und Zuordnung der Effekte aufwirft. Anschließend werden das Berechnungsverfahren vorgestellt und die verschiedenen Arten von Ertragsraten diskutiert.

\subsubsection{Bildung als Investition oder Konsum}

Die Bedeutung der Bildung für die Entwicklung der Fähigkeiten eines Menschen führt dazu, Bildungsaufwendungen als Investition zu betrachten. Investitionen sind Aufwendungen in der Gegenwart, die in der Absicht getätigt werden, die Erträge in der Zukunft zu erhöhen. Ein wesentliches Element dabei ist die Gewinnerwartung des Investors.

Bezüglich der Bildung erhebt sich allerdings die Frage, ob alle Ausgaben als Investition zu betrachten sind, oder ob nicht zumindest ein Teil der Aufwendungen Konsumcharakter aufweisen. Grundsätzlich lässt sich sagen, dass Bildungsaufwendungen sehr oft dualen Charakter besitzen. Dies soll anhand der folgenden Grafik dargestellt werden.

20 Pionierarbeit bezüglich der Bewertung von Humankapitalinvestitionen leisteten: Bowman (1962), Merrett (1966), Schultz (1961) und Becker (1983). 
SCHULTZ gliedert den Charakter von Bildungsaufwendungen folgendermaßen²1:

Abbildung 2.1: Bildung als Investitions- und Konsumgut

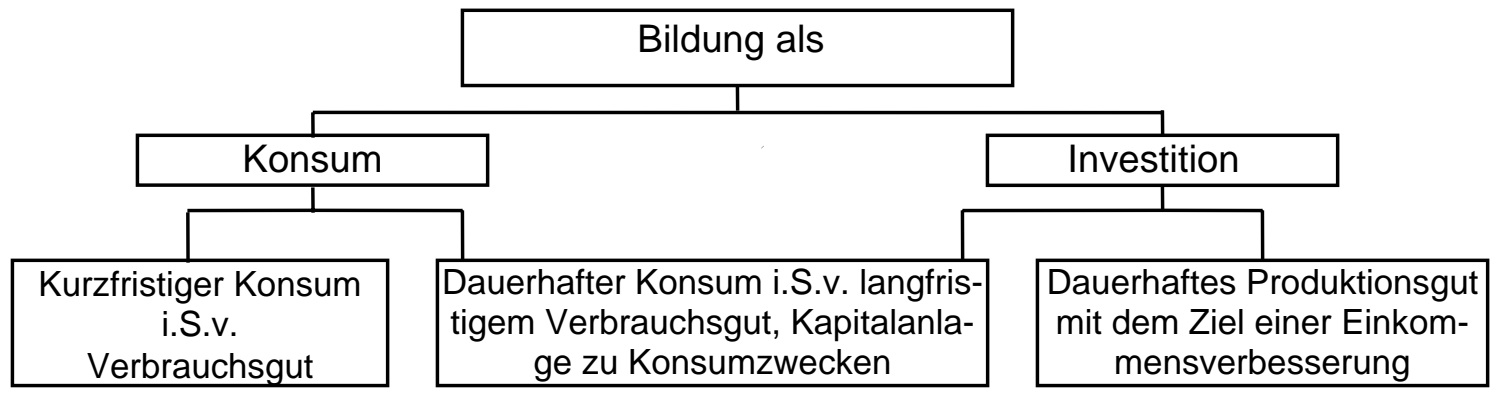

Quelle: Schulz (1963): The Economic Value of Education, S.8.

Die Konsumkomponente beinhaltet zunächst den kurzfristigen Konsum (also z.B. der Genuss des „Studentenlebens“) aber auch den zukünftigen Konsum (wie zum Beispiel die verbesserte Kapazität, ein Buch zu genießen). Fällt der Nutzen in der Zukunft an, hat die Bildung auch die Eigenschaft einer Investition. Als Investition im engeren Sinne gelten die Bildungsausgaben aber nur, wenn sie eine Steigerung der zukünftigen Einkommen bewirken. Die Bildungsausgaben sind dann mit Investitionen in Produktionsgüter vergleichbar.

Wie die Grafik verdeutlicht, ist eine eindeutige Trennung der beiden Komponenten nicht möglich. Um die möglichen Effekte der Bildung zu ermitteln, müssen die Kosten und Erträge der Ausbildung bekannt sein. Der folgende Abschnitt gibt daher einen Einblick in die Vielfalt der möglichen Kosten und Nutzen von Bildungsinvestitionen und zeigt die bereits erwähnten Bewertungsprobleme auf.

21 Vgl. Schultz (1963), 1963, S. 8. 


\subsubsection{Kosten und Nutzen von Bildungsinvestitionen}

Die Kosten von Ausbildungsinvestitionen sind relativ einfach zu ermitteln. Auf der Kostenseite der privaten Kalkulation gehen neben den direkten Kosten, die die Familien direkt aus ihren Einkommen zu finanzieren haben, auch noch die Opportunitätskosten des Schulbesuchs ein. Die direkten Kosten setzen sich aus Schulgebühren, Ausgaben für Schuluniformen und Unterrichtsmaterialien und Fahrtkosten zusammen. Die Opportunitätskosten sind die Einkommen, die den Schülern entgehen, weil sie zugunsten des Schulbesuchs auf Verdienstmöglichkeiten verzichten. Diese Opportunitätskosten werden üblicherweise aus den Einkommen berechnet, die die Absolventen des nächsten, niedrigeren Abschlusses am Arbeitsmarkt erhalten. Sie sind aus Alters-Einkommens-Profilen zu ermitteln. Wird als Alternative zum Schulbesuch eine Beschäftigung am Arbeitsmarkt für unrealistisch erachtet, können dennoch erhebliche Opportunitätskosten entstehen, weil die Kinder nicht mehr als Arbeitskraft im eigenen Unternehmen oder im Haushalt zur Verfügung stehen. Die hieraus entstehenden Kosten werden üblicherweise aus den Ausgaben für Haushaltsangestellte oder dem Verdienstausfall von anderen Familienmitgliedern, die statt dessen zu Hause bleiben müssen, berechnet.

Vorstellbar sind aber auch nicht-monetäre negative Effekte der Schulbildung, die bei der Bildungsentscheidung eine Rolle spielen könnten. Eltern könnten z.B. Angst um ihre Kinder haben, wenn sie in den Schulen eine gewalttätige Atmosphäre vermuten. Auch die Konfrontation der Kinder mit politischen Meinungsäußerungen oder einer unerwünschten sexuellen Freizügigkeit unter den Schülern kann die Bildungsentscheidung negativ beeinflussen. ${ }^{22}$ Diese Effekte werden bei der Ermittlung von Ertragsraten aber üblicherweise vernachlässigt.

Für die gesamtgesellschaftliche Kalkulation kommen zu den privaten Kosten noch die staatlichen Ausgaben für den Bildungssektor hinzu.

Die Erträge von Ausbildungsinvestitionen sind in ihrer Art weiter gefächert und oft schwerer zu bewerten. Eine Darstellung der möglichen Erträge soll nach der Systematisierung von WEIßHUHN erfolgen. ${ }^{23} \mathrm{Er}$ unterscheidet zunächst zwischen internen und externen Effekten von Bildungsinvestitionen. Die internen Effekte begründen den privaten Wert einer Investition, weil sie dem Investor selbst entstehen. Die externen Effekte können einen begrenzten Personenkreis und auch

22 Dieser Effekt kann sich aber auch positiv auswirken, wenn die Schule zum Beispiel als Möglichkeit betrachtet wird, die Kinder von der gefährlicheren Straße zu bekommen. Vgl. Birdsall (1985), S. 71.

23 Vgl. Weißhuhn (1977), S. 120-138. Eine ausführliche Beschreibung der Konsumeffekte und sonstiger Effekte von Bildung und Hinweise auf empirische Überprüfungen liefert McMahon (1987a). 
die Gesellschaft als ganzes betreffen. Je nach Entscheidungseinheit wirken sich die externen Effekte auch auf die private oder nur auf die gesamtgesellschaftliche und damit politische Entscheidung aus. Für die Bestimmung des sozialen Wertes der Ausbildung müssen natürlich die gesamten Effekte berücksichtigt werden.

Als interne Effekte, die sich auf das investierende Individuum auswirken, wird die folgende Einteilung vorgeschlagen:

Abbildung 2.2: Interne Effekte von Bildungsinvestitionen

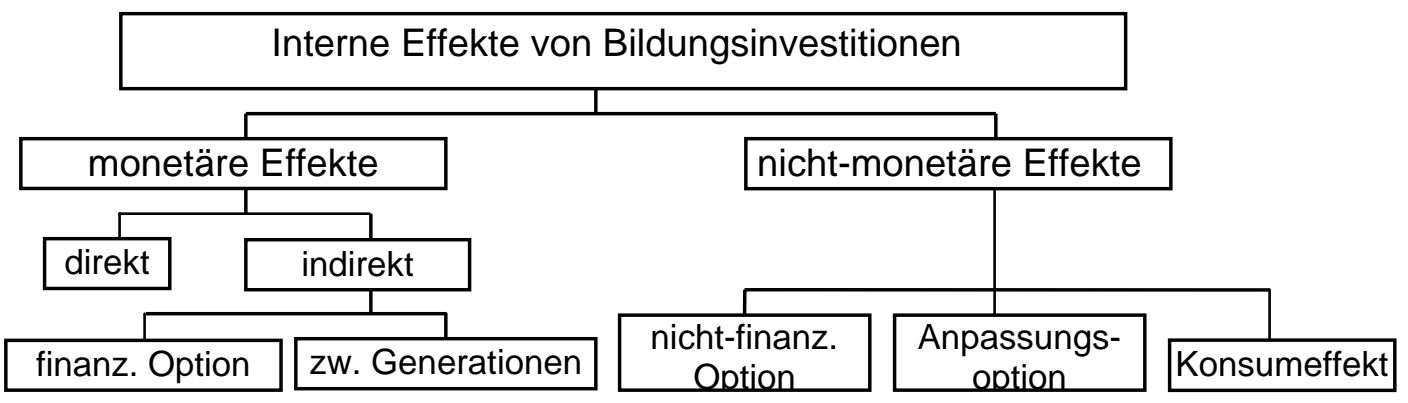

Quelle: Weißhuhn (1977), S. 120-127, eigene Darstellung.

Zunächst werden die Effekte für den privaten Investor in monetäre und nichtmonetäre Effekte eingeteilt. Der direkte monetäre Effekt ist der Einkommenszuwachs, den die zusätzliche Ausbildung induziert. ${ }^{24}$ Theoretisch müsste dieser aus den Einkommensdifferenzen von Personen mit unterschiedlichem ermittelt werden. Ökonomen ziehen dazu Alters-Einkommens-Profile heran. ${ }^{25}$

Neben diesen direkten Einkommenseffekten können noch indirekte monetäre Effekte auftreten. Sie bestehen aus der reinen Möglichkeit, sich weitere Einkommen zu erschließen oder wirken sich erst bei folgenden Generationen auf die Einkommen aus. Sie sind nur schwer quantifizierbar und kaum eindeutig den Bildungsinvestitionen zuzurechnen. Solch einen finanziellen Optionsnutzen induziert die Ausbildung z.B., wenn sie die Voraussetzung für weiterführende Bildung darstellt oder weitere berufliche Aufstiegschancen ermöglicht. Damit hat sich der Investor die Möglichkeiten für weitere Einkommenszuwächse erschlossen. Von einem intergenerativen Effekt wird insofern gesprochen, als sich die zusätzliche Bildung des Investors später auch auf dessen Kinder auswirkt, indem er sie beim Lernen unterstützt, den Berufseinstieg erleichtert und sie somit höhere Einkommen erreichen können.

24 Aus der Sicht des Individuums wird hier nur das Nettoeinkommen berücksichtigt.

25 Die Probleme die dabei entstehen, werden in Abschnitt 2.1.4 über die verschiedenen Arten von Ertragsarten behandelt. 
Neben diesen Effekten, die Einkommenszuwächse begründen (können), kann die Ausbildung auch Nutzen nichtmonetärer Art generieren. Sie sind noch schwieriger zu bewerten als die monetären Effekte. Hier handelt es sich zunächst um die erweiterten beruflichen Möglichkeiten einer zusätzlichen Ausbildung. So kann z.B. ein Beruf gewählt werden, der mehr Freiheit, Selbständigkeit oder Prestige verspricht und damit die Zufriedenheit des Individuums erhöht. Ein weiterer nicht-monetärer Effekt ist die verbesserte Möglichkeit, sich an sich verändernde Strukturen oder Technologien anzupassen und damit das Risiko auf Arbeitslosigkeit zu reduzieren. Letztendlich ermöglicht die Ausbildung auch die verbesserte Nutzung der Freizeit, des kulturellen Angebotes und eine effizientere Haushaltsführung und hat damit auch einen Konsumeffekt. ${ }^{26}$

Diese Wirkungen betreffen den Nutzen des Individuums selbst. Sofern sie sich indirekt auswirken oder nicht-monetärer Art sind, treten bei der bildungsökonomischen Analyse Bewertungs- und Zuordnungsprobleme auf. Dennoch werden diese Effekte bei der individuellen Bildungsentscheidung eine Rolle spielen. Ihren exakten Wert beim privaten Kalkül anzusetzen, ist aber kaum möglich.

Ein weiteres Phänomen der Ausbildung sind die externen Nutzen. Unter externen Effekten einer Investition versteht man Wirkungen auf unbeteiligte Dritte oder die Gesellschaft als Ganzes, die nicht das investierende Individuum betreffen und deshalb nicht in das private Investitionskalkül eingehen.

\section{Abbildung 2.3: Externe Effekte von Bildungsinvestitionen}

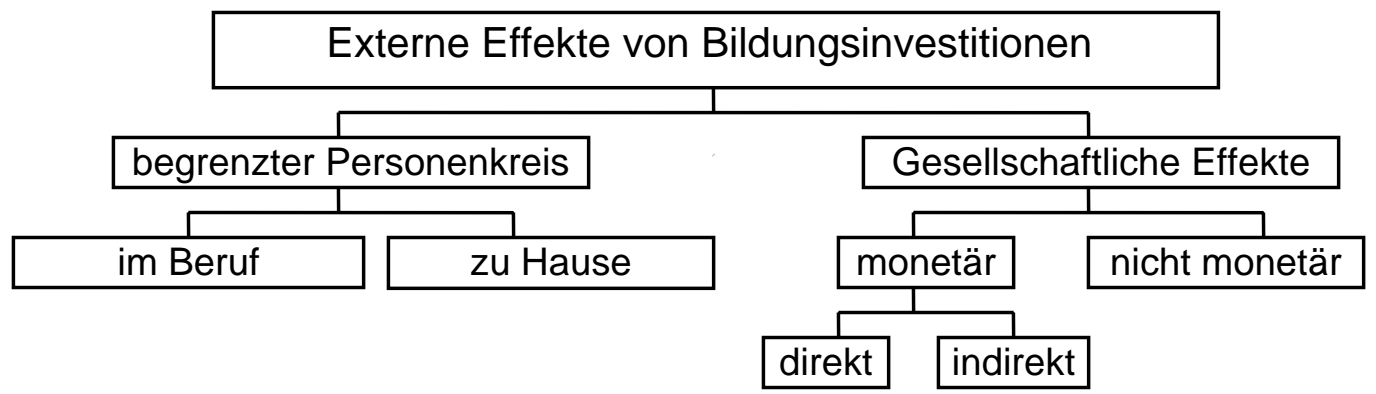

Quelle: Weißhuhn (1977), S. 128-138, eigene Darstellung.

Externe Effekte der Ausbildung, deren Wirkung auf einen Personenkreis beschränkt bleibt, treten nach WEIßHUHN ${ }^{27}$ einmal im Beruf auf und darüber hinaus noch in der eigenen Familie. Im beruflichen Umfeld wird z.B. erwartet, dass die Produktivität auch bei den Mitarbeitern steigt, die mit der ausgebildeten Person direkt zusammenarbeiten. Die Beteiligten an Gruppen- oder Teamarbeiten werden von der Leistungsfähigkeit des ausgebildeten Kollegen motiviert und profi-

26 Vgl. auch Psacharopoulos (1984), S. 347.

27 Vgl. Weißhuhn (1977), S. 128-129. 
tieren selbst von Lerneffekten. Im Haushalt kann vor allem die Aufsichtsfunktion der Schule positive Auswirkungen haben. Kann z.B. eine Mutter durch den Schulbesuch des Kindes wieder in ihren Beruf einsteigen, ist der somit induzierte Einkommenseffekt ebenfalls eine Auswirkung der Schulbildung.

Die Auswirkungen einer zusätzlichen Ausbildung auf die Gesellschaft als Ganzes zeigen sich durch die Beeinflussung der staatlichen Aufgaben und der sozialen Wohlfahrt. ${ }^{28}$ Als direkte monetäre gesellschaftliche Effekte sind die zusätzlichen Steuern zu nennen, die die Ausgebildeten wegen ihrer höheren Einkommen zahlen müssen. ${ }^{29}$ Sie lassen sich aus dem Steuertarif relativ leicht ermitteln.

Indirekte monetäre Effekte entstehen aus vermiedenen Kosten. Zum Beispiel wird angenommen, dass die Schule einen Erziehungsanspruch erfüllt, der die Kriminalität sinken lässt. Die so gesparten Ausgaben für die öffentliche Sicherheit wären somit ein Ertrag der Ausbildung. Wird die Unterstützung von Arbeitslosen aus staatlichen Kassen finanziert, ergibt sich auch hier eine gesamtgesellschaftliche Wirkung der Ausbildung. Dazu wird angenommen, dass besser ausgebildete Arbeitskräfte flexibler auf Veränderungen am Arbeitsmarkt reagieren können und deshalb seltener arbeitslos werden. 30 Ähnliche Auswirkungen werden auf die Gesundheitssituation der Bevölkerung und damit auf die Kosten eines öffentlichen Gesundheitssystems erwartet. Obwohl es sich hierbei um monetäre Effekte handelt, sind sie nur schwer quantifizierbar, da ihr Ausmaß kaum vorausgesagt werden kann. Selbst wenn Einsparungen in den genannten Bereichen zu beobachten sind, ist nur schwer zu ermitteln, in welchem Maß sie der verbesserten Ausbildung zuzuordnen sind.

Noch schwieriger lassen sich die nicht-monetären Effekte der Ausbildung auf die Gesellschaft quantifizieren. Zum Beispiel wird mit höherer Bildung eine höhere Wertschätzung und damit Schonung der Umwelt erwartet. ${ }^{31}$ Die verbesserte Umweltqualität erhöht den eigenen Nutzen und den Nutzen zukünftiger Generationen. Dieser Effekt muss der Ausbildung zugeordnet werden. Ebenso wurde der Ausbildung eine positive Wirkung auf die Einkommensverteilung nachgewiesen. ${ }^{32}$ Eine besser ausgebildete Bevölkerung trägt zum besseren

28 Vgl. Weißhuhn (1977), S. 130-138.

29 Die Einkommensteuern sind streng genommen Teil des Einkommenseffektes der Ausbildung. Die Steuereinnahmen selbst sind nur eine Umverteilung eines Teils der induzierten Einkommens. Da sie nicht in das Kalkül des Individuums eingehen, sind sie hier den externen Wirkungen zugeordnet. Ihre Bedeutung für die Unterscheidung von Ertragsraten erfolgt weiter unten.

Einen Nachweis dazu für Spanien erbrachten Mauro/Spilimbergo (1999), S. 1-17.

31 Vgl. McMahon (1987b). Hier werden die externen Effekte der Ausbildung beschrieben und Hinweise zu empirische Überprüfungen gegeben.

32 Eine Zusammenfassung der frühen Literatur dazu liefert Psacharopoulos (1984), S. 347. 
Funktionieren der Institutionen eines demokratischen Staates bei und fördert eine effizientere Gestaltung des öffentlichen Lebens. Die Informationsverbreitung durch gut ausgebildete Individuen kann als gesellschaftliche Qualifikation angesehen werden. Anhand dieser Beispiele wird deutlich, wie schwierig eine Bewertung und Zuordnung der Bildungseffekte sein kann.

\subsubsection{Das Berechnungsverfahren}

Untersuchungen über die Effizienz von Bildungsinvestitionen können über zwei Wege erfolgen: die Methode anhand von Alters-Einkommens-Profilen und die Methode anhand von Einkommensfunktionen. ${ }^{33}$ Die Verwendung von AltersEinkommens-Profilen stellt die vorzuziehende Methode für die Berechnung von Ertragsraten dar, wenn aktuelle Daten zur Einkommensgeschichte der Individuen verwendet werden. Allerdings ist diese Methode sehr datenintensiv. Ausreichendes Datenmaterial für vollständige Einkommensprofile ist in den meisten Ländern nicht vorhanden. Deshalb sind einige Autoren dazu übergegangen, die Erträge einer Ausbildung anhand von Einkommensfunktionen zu schätzen. Dabei wird meistens ${ }^{34}$ von der Einkommensfunktion nach MINCER (1974) ausgegangen. ${ }^{35}$ Üblicherweise wird angenommen, dass das logarithmierte Einkommen eines Individuums $\left(w_{i}\right)$ von dessen Schulbildung ${ }^{36}\left(S_{i}\right)$ und Erfahrungen $\left(E_{i}\right)$ abhängt. Andere das Einkommen beeinflussende Faktoren werden durch $u_{i}$ repräsentiert. 37

$$
\ln \left(w_{i}\right)=f\left(S_{i}, E_{i}, u_{i}\right)=a_{0}+a_{1} S_{i}+a_{2} E_{i}+a_{3} E_{i}^{2}+u_{i}
$$

Erweiterungen lassen auch die Berücksichtigung von weiteren Faktoren und die Berechnung von Koeffizienten für einzelne Bildungsstufen zu. Die Koeffizienten werden häufig als private Ertragsraten bezeichnet, obwohl es sich eigentlich um den marginalen Lohneffekt der Ausbildung handelt. Sie müssen zu den entsprechenden Kosten der Ausbildung in Beziehung gesetzt werden, um als Ertragsrate interpretiert werden zu können. ${ }^{38}$

Da es sich bei den Ertragsratenberechnungen anhand von Einkommensfunktionen nur um einen zweitbesten Ansatz handelt, wenn die Datenlage schlecht ist

Vgl. zu den beiden Methoden die Ausführungen bei Psacharopoulos (1994), S. 1325-1326.

Vgl. Psacharopoulos (1994), S. 1325. Vgl. auch Björklund/Kjellström (1999), S. 2, Wright (1999), S. 4 , Glewwe (1996), S. 269 und Siphambe (1999), S. 292.

35 Vgl. Mincer (1974), S. 83-96.

36 Meistens wird die Schulbildung anhand der abgeschlossenen Schuljahr gemessen.

37 Vgl. Glewwe (1996), S. 269. Vgl. auch Wright (1999), S. 292.

38 Jeder Bildungskoeffizient muss zu dem Koeffizienten der vorherigen Bildungsstufe in Beziehung gesetzt und durch die Anzahl der zusätzlichen Jahre geteilt werden. Vgl. Psacharopoulos (1994), S. 1326. Für eine kritische Diskussion des Mincer-Ansatzes am Beispiel Schwedens vgl. Björklund/Kjellström (1999).
} 
oder der Aufwand der Datenbeschaffung gescheut wird, soll im folgenden nur die Methode anhand von Alter-Einkommens-Profilen detailliert dargestellt werden. Sie eignet sich auch besser, um das Prinzip der Ertragsratenberechnung darzustellen. Bei dieser Art der Berechnung, die eine Bewertung der Effizienz von Bildungsinvestitionen erlaubt, werden die Kosten und die Erträge der Ausbildung gegenübergestellt.

Wie die obigen Ausführungen gezeigt haben, lassen sich die Aufwendungen für Ausbildung nicht eindeutig in investive und konsumtive Komponenten zerlegen. Außerdem sind die Nutzen oft weder eindeutig zuzuordnen noch exakt monetär zu bewerten. Aufgrund dieser Schwierigkeiten werden zur Ermittlung von Ertragsraten von Bildungsinvestitionen nur die direkten monetären Erträge berücksichtigt ${ }^{39}$ und den gesamten Kosten Investitionscharakter zugeschrieben.

Den gesamten Bildungsaufwendungen steht also nur ein Teil der Erträge gegenüber. Dieses Vorgehen hat den Vorteil einer eindeutigen Zurechenbarkeit und vermindert die Gefahr einer willkürlichen Beeinflussung der Ergebnisse. Bei der Interpretation der Ertragsraten sollte allerdings berücksichtigt werden, dass sie einen Teil der Nutzen nicht berücksichtigen. ${ }^{40}$

Zur Bewertung von Bildungsinvestitionen wurden die Verfahren aus der Investitionsrechnung herangezogen. Obwohl in der Investitionsrechnung die Aussagefähigkeit der internen Zinsfußmethode sehr unterschiedlich bewertet wird ${ }^{41}$, hat sie sich in der Bildungsökonomie durchgesetzt. Sie beschreibt den Zusammenhang zwischen Kosten und Erträgen eines Investitionsprojektes. Der interne Zinsfuß entspricht dem Diskontfaktor, der den Gegenwartswert des Nettonutzenstroms gleich Null setzt. Dieser Zins wird in der Bildungsökonomie als Ertragsrate bezeichnet. Die so errechneten Ertragsrate ist also der Zins, bei dem der Gegenwartswert der pekuniären Kosten dem Gegenwartswert der pekuniären Nutzen entspricht. 42

Die Berechnung soll symbolisch für eine Bildungsinvestition dargestellt werden. ${ }^{43}$ Angenommen, die Investition besteht darin, durch vier weitere Schuljahre den nächsthöheren Abschluss nach der Sekundarstufe zu erreichen. Die Kosten während dieser zusätzlichen Schuljahre bestehen aus direkten Ausgaben $\left(C_{h}\right)$ und verlorenen Einkommen $\left(W_{s}\right)$. Die Nutzen bestehen hauptsächlich aus

\footnotetext{
39 Vgl. Psacharopoulos (1973), S. 20.

40 Vgl. Psacharopoulos (1973), S. 32.

41 Vgl. Busse von Colbe/Lassmann (1990), S. 105-127.

42 Vgl. Moonwon Kang (1993). Der Autor zeigt, dass der Konsumnutzen der Bildung in entwickelten Ländern höher ist als in Entwicklungsländern und daher ein Ländervergleich der pekuniären Ertragsraten nicht zulässig ist.

43 Vgl. Psacharopoulos (1973), S. 20-22.
} 
der Differenz der Einkommen von Absolventen der höheren Ausbildungsstufe $\left(W_{h}\right)$ und denen der Absolventen der Sekundarstufe $\left(W_{s}\right)$. Wenn die Ausbildung vier Jahre dauert und der Absolvent 43 Arbeitsjahre vor sich hat, kann die Ertragsrate der Investition in 'höhere Bildung' ermittelt werden, indem die folgende Gleichung nach $r$ aufgelöst wird.

$$
\sum_{t=-3}^{0}\left(C_{h}+W_{s}\right)_{t}(1+r)^{-t}=\sum_{t=1}^{43}\left(W_{h}-W_{s}\right)_{t}(1+r)^{-t}
$$

Alle Kosten werden auf den Zeitpunkt 0 aufdiskontiert, und alle Erträge werden auf eben diesen Zeitpunkt abdiskontiert. Dieses Vorgehen hat den Vorteil, den Nettonutzen in einen Kosten- (linke Seite) und einen Nutzenstrom (rechte Seite) zerlegen zu können. Eine Analyse der Einflussfaktoren wird damit vereinfacht.

Die Ertragsrate ist demnach der Zins, bei dem die aufgezinsten Kosten genau den abgezinsten Nutzen entsprechen. Auf diese Weise kann eine Reihe von Ertragsraten für verschiedene Bildungsniveaus ermittelt werden. Üblicherweise werden die Ertragsraten für die drei Stufen Primar-, Sekundar- und höhere Bildung ermittelt. Sie können aber auch für verschiedene Schultypen innerhalb einer Stufe ermittelt werden.

Je nach Erkenntnisinteresse werden unterschiedliche Bestandteile in die Berechnung einbezogen. Die so entstehenden Arten von Ertragsraten werden im folgenden dargestellt. 


\subsubsection{Ertragsratenarten}

Die obige Gleichung ist in allgemeiner Form gegeben. Je nachdem welche Variablen in der Gleichung berücksichtigt werden, werden verschiedene Ertragsraten ermittelt. 44

Zunächst ist die Unterscheidung in private und soziale Ertragsraten zu nennen. Zur Ermittlung privater Ertragsraten werden nur die Effekte der Ausbildung berücksichtigt, die das investierende Individuum betreffen. Sie bestimmen also den Wert der Bildung für das Individuum und können daher als Einflussfaktor der privaten Nachfrage betrachtet werden. Da die Individuen natürlich keine derart exakte Abwägung von Kosten und Erträgen vornehmen und dies auch aufgrund von Informationsmangel gar nicht könnten, sind die privaten Ertragsraten eher ein Instrument der Ökonomen zur Formalisierung des angenommenen subjektiven Kalküls.

Soziale Ertragsraten ermitteln den Wert der Bildung für die Gesellschaft und sind bei der öffentlichen Bildungsplanung relevant. Sie können herangezogen werden, um die Frage zu klären, wie die öffentlichen Ressourcen zwischen Bildung und anderen Investitionsformen oder zwischen den verschiedenen Bildungsformen alloziiert werden sollen.

Auf der Kostenseite unterscheiden sich die beiden Raten durch die Bereitstellungskosten, die von der öffentlichen Hand getragen werden. Der Schüler zahlt in der Regel nur einen geringen Teil der Kosten seiner Ausbildung. In die soziale Rate gehen alle anfallenden Kosten der Bildung ein.

Auf der Nutzenseite unterscheiden sie sich durch die Steuereinnahmen, die durch höhere Einkommen induziert werden. In die private Ertragsrate gehen nur die Nettoeinkommen ein. Da in der Regel die zusätzlichen Steuern niedriger sind als die Kostendifferenz, müssten die privaten Ertragsraten über den sozialen liegen.

Soziale Ertragsraten sind für die individuelle Bildungsentscheidung natürlich zunächst irrelevant. Allerdings spielen sie eine große Rolle, wenn die private Bildungsnachfrage staatlich beeinflusst werden soll. Sind zum Beispiel die sozialen Raten - aufgrund von Informationsmängeln oder der Berücksichtigung der externen Effekte - höher als die privaten, ist Anlass zu staatlichem Handeln gegeben, da die private Nachfrage in diesem Fall hinter dem gesellschaftlich gewünschten Niveau zurückbleiben würde. Ein Vergleich der beiden Ertragsraten

44 Zu den folgenden Ausführungen vgl. Psacharopoulos (1973), S. 20-24. 
wird auch zur Diskussion einer stärkeren privaten Beteiligung an der Bildungsfinanzierung herangezogen. ${ }^{45}$

Eine weitere Unterscheidung wird in der Literatur zwischen marginalen und durchschnittlichen Ertragsraten vorgenommen. Eine marginale Ertragsrate im engeren Sinne müsste die Effekte eines zusätzlichen Schuljahres auf der Kosten- und Ertragsseite berücksichtigen. In der Realität werden Bildungsentscheidungen aber nicht für jedes einzelne Jahr sondern jeweils für die gesamte nächste Bildungsstufe getroffen. Traditionell werden daher in der Bildungsökonomie marginale Ertragsraten für ein bestimmtes Bildungsniveau ermittelt. Dabei werden die Einkommensdifferenzen zwischen Absolventen einer Bildungsstufe und den Absolventen der nächst niedrigeren Stufe herangezogen. Noch in einem anderen Sinn können die so ermittelten Ertragsraten als "marginal“ verstanden werden: Sie gelten immer nur für einzelne oder eine kleine Gruppe von Individuen. Wird ein bestimmtes Bildungsniveau im großen Rahmen ausgeweitet und drängen die Absolventen mit diesem Abschluss geballt auf den Arbeitsmarkt, werden die Löhne und damit wiederum die Ertragsraten beeinflusst. In der Literatur werden marginale Ertragsraten also in dem Sinne verstanden, dass sie für eine zusätzliche Bildungsstufe und eine begrenzte Anzahl von Individuen gelten.

Über diese stufenweise Betrachtung hinaus ist es möglich, die AltersEinkommens-Profile von Schulabsolventen mit denen von Menschen zu vergleichen, die gar keine Schule besucht haben. Die so ermittelten Ertragsraten nennt man Durchschnittsraten. Sie stellen den Wert der Ausbildung gegenüber der Alternative 'keine Ausbildung' dar. Sie werden eher zu Informationszwecken als zur Unterstützung von Investitionsentscheidungen ermittelt und haben in der bildungsökonomischen Literatur kaum Relevanz.

Bei der Interpretation von Ertragsraten ist weiterhin zu unterscheiden, ob innen eine ex post- oder eine ex ante- Analyse zugrunde liegt. Diese Angaben wurden in der Literatur selten explizit gemacht, was zu Verwirrung führen kann. Beide Formen von Ertragsraten sind für unterschiedliche Aussagen geeignet. Eine Unterscheidung ist allerdings nur in der Theorie eindeutig möglich, da in der Praxis kaum die dafür notwendigen Daten vorliegen.

Im Idealfall sollte für die Ermittlung einer ex post-Ertragsrate eine gegebene Kohorte über die Zeit verfolgt werden. Die so ermittelten Zeitreihen über das Einkommen nach dem Bildungsniveau sollten die Grundlage für die Ertragsratenberechnung bilden. Diese Art der Ertragsraten gibt Aufschluss über die Effi- 
zienz der in der Vergangenheit getätigten Bildungsinvestition. Sie bietet aber kaum Informationen über den individuellen Entscheidungsprozeß.

Die ideale ex ante-Ertragsrate sollte auf der Basis von zukünftigen Einkommensprofilen der heutigen Schüler errechnet werden. Es handelt sich dabei um erwartete (zukünftige) Erträge der heute getätigten Bildungsinvestition. Eine so errechnete private Ertragsrate wäre die relevante Größe für die optimale private Bildungsentscheidung.

Gäbe es verlässliche Daten zu den Ertragserwartungen in der Bevölkerung, könnten diese Ertragsraten zur Analyse von Investitionsentscheidungen herangezogen werden. So könnte zum Beispiel untersucht werden, wie sich die Individuen bezüglich einer Veränderung der erwartetet Ertragsrate verhalten.

In der Praxis sind selbst die notwendigen Zeitreihen-Daten für ex post-Analysen nur selten verfügbar. Die zukünftige Einkommensentwicklungen kann weder von Ökonomen und noch viel weniger von den Individuen genau vorausgesagt werden. Deshalb werden Ertragsraten üblicherweise auf der Basis von aktuellen Querschnittsdaten berechnet. Aus aktuellen Erhebungen werden Kreuztabellen nach Geschlecht, Alter, Einkommen und Bildungsstand (wenn möglich, werden weitere Kriterien herangezogen) gebildet. Aus diesen Tabellen lassen sich dann die so genannten Alters-Einkommens-Profile für verschieden Bildungsabschlüsse ermitteln, die die Grundlage für Ertragsberechnungen bilden.

Die Einkommensunterschiede bei verschiedenen Bildungsniveaus zu einem Zeitpunkt werden dann als Maßstab für die Einkommensentwicklung eines Schulabsolventen interpretiert. Je nach Erklärungsbedarf werden die Daten dann vor- oder zurückextrapoliert, um ex ante- bzw. ex post-Maße für die Bildungsprofitabilität zu erhalten. 


\subsection{Kritik am Konzept der Ertragsraten}

Das Ertragsratenkonzept wird in der Literatur kontrovers diskutiert. Als populärster Einwand ist die sogenannte "Screening"-Hypothese zu nennen. Diese Kritik richtet sich generell an die Ansätze, die Ausbildung als Investition betrachten. Sie sagt aus, dass Produktivitäts- und Einkommensunterschiede auf unterschiedlichen (angeborenen) Fähigkeiten basieren und kein direkter Effekt der Schulbildung sind. ${ }^{46}$ Die erworbenen Schulabschlüsse dienen nach dieser Theorie nur zur Identifizierung der fähigeren Arbeitskräfte. ${ }^{47}$ Allerdings konnte auch im Rahmen dieser Kritik kein kostengünstigerer Auswahlmechanismus vorgestellt werden.

Angesichts einiger misslungener Testversuche ${ }^{48}$ gilt die Screening-Hypothese heute eher als Warnung vor einer Überbewertung der ermittelten sozialen Ertragsraten ${ }^{49}$ und weniger als eine empirisch fundierte Widerlegung des Humankapitalansatzes. 50

Ein zweiter Kritikansatz richtet sich direkt an das Ertragsratenkonzept. Hier wird bemängelt, dass nur die direkten und monetär messbaren Erträge der Ausbildung in das Kalkül eingehen. Gerade für Entwicklungsländer seien die weiteren Effekte von großer Bedeutung. Hier werden z.B. die verbesserte Einhaltung von Hygienevorschriften und die damit verbundene besserer Gesundheitsvorsorge, die verminderte Geburtenrate oder eine effizientere Haushaltsplanung genannt. Andererseits ist die Ertragsrate der entwickelten Länder unterbewertet, wenn der Ausbildung auch ein konsumtiver Nutzen zuerkannt wird und sie als superiores Gut verstanden wird. ${ }^{51}$ Für Entwicklungsländer ergibt sich ein weiterer Kritikpunkt. Wenn die Ertragsraten nur aufgrund von Einkommensdifferenzen ermittelt werden, haben sie für einen großen Teil der Bevölkerung keine Aussagekraft. In Entwicklungsländern bestreiten viele Menschen ihren Lebensunterhalt im Nicht-Lohnsektor und durch Landwirtschaft. Einige Untersuchungen im ländlichen Bereich ermittelten den Zusammenhang zwischen Ausbildung und

46 Vgl. Stiglitz (1975), S. 283-300 und Winkler (1987), S. 287-291.

47 Zur Rolle der Zeugnisse als Auswahlkriterium vgl. Van der Meer/Wielers (1996), S. 29-46.

48 Hungerford/Solon konnten zwar einen Sheepskin-Effekt anhand ihrer Daten nachweisen, betonen aber, dass damit die Screening Hypothese keineswegs bestätigt ist. Vgl. Hungerford/Solon (1987), S. 175-177.

49 Denison (1964) hat ermittelt, dass nur zwei Drittel der Einkommen auf die Ausbildung zurückgehen. Die restlichen Einkommen sind Resultat von angeborenen Fähigkeiten, familiärem Hintergrund etc. Er schlägt vor, die Einkommen mit einem $\alpha$-Koeffizient ( $\alpha$ für ability) in Höhe von 0,66 zu multiplizieren.

50 Vgl. Psacharpoulos (1996), S. 342-343. Weiss betrachtet die „Sorting Models“, die sowohl Screening als auch Signaling berücksichtigen eher als eine sinnvolle Ergänzung des Humankapital-Ansatzes als eine Widerlegung desselben. Die verbreitete Ablehnung der Sorting Models basiert seiner Meinung nach auf der Unkenntnis dieses Sachverhalts. Vgl. Weiss (1995), S. 134, 150-151. 
agrarischer Effizienz bzw. Produktivität. 52 Diese Untersuchungen, die Produktivitätssteigerungen mittels des landwirtschaftlichen Outputs bestimmen, ermittelten einen positiven Effekt der Schulbildung eines Farmers auf seine Produktivität und den Einsatz neuer Technologien. ${ }^{53}$ Allerdings gibt es auch Untersuchungen mit gegenteiligen Ergebnissen. ${ }^{54}$

Ähnliche Kritik wird wegen der Behandlung der externen Effekte vorgebracht. Bei Nutzen-Kosten-Analysen sind sie der Hauptfaktor für die Diskrepanz zwischen privaten und sozialen Berechnungen. Das Auftreten externer Effekte kann die staatliche Subventionierung von privaten Investitionen rechtfertigen. In die sozialen Ertragsraten von Humankapitalinvestitionen geht zwar der öffentliche Anteil an den Kosten in die Berechnung ein, aber die externen Erträge werden nur zum Teil berücksichtigt.

Um diese Art von Effekten in die Berechnung einzubeziehen, müssen innen monetäre Werte beigemessen werden. Wie die obigen Ausführungen zeigten, ist dies aber nicht eindeutig möglich. Die Bewertung bietet subjektiven Spielraum und macht die Ergebnisse beliebig beeinflussbar. Eine bessere Alternative zur Berücksichtigung dieser Effekte ist ihre Aufführung neben der herkömmlichen Ertragsraten-Berechnung. So können sie bei der Interpretation der Ertragsraten und zur Unterstützung von Investitionsentscheidungen offen herangezogen werden.

Ein dritter Ansatzpunkt für Kritik besagt, dass selbst die direkten monetären Effekte von Ausbildung nicht eindeutig erfasst werden können. Querschnittsdaten können nur eine vage Annäherung an die relevanten Einkommensdaten sein. Zahlreiche nicht näher bestimmbare Veränderungen (Wirtschaftswachstum, Strukturwandel, Steuerreformen usw.) lassen eine genaue Vorausschau auf die zukünftige Einkommensentwicklung nach Bildungsniveaus nicht zu. Eine auf Ertragsraten basierende Bildungspolitik kann sich unter den veränderten Bedingungen als falsch erweisen.

Die notwendigen Anpassungen und Projektionen lassen selbst bei den direkten monetären Werten noch Bewertungsspielräume zu. Dadurch wird die Vergleichbarkeit von Ertragsraten vermindert. Außerdem wird ein Vergleich von Ertragsraten verschiedener Ländern dadurch erschwert, dass ihnen unter-

52 Vgl. Lockheed/Jamison/Lau (1980), S. 111-152.

53 Eine Auflistung einiger Studien und deren Ergebnisse findet sich bei Psacharopoulos (1994), S.1328.

54 Vgl. Moock (1981), S. 723-739. Dieses unerwartete Ergebnis auf die Maisproduktion in Kenia wurde allerdings nur für Farmer ermittelt, die die Schule vor dem Primarschulabschluss verlassen haben. Für Absolventen der Primaria ermittelte auch Moock positive Effekte. Vgl. dazu Lipton (1985), S. 167168 und Moock (1985), S. 169-172. 
schiedliche Bildungssysteme und damit verschiedene Bildungsstufen zugrunde liegen.

Trotz dieser Kritik wurden für zahlreiche Länder Ertragsraten für verschiedene Bildungsstufen ermittelt. Einige Ergebnisse der Berechnungen sollen im folgenden vorgestellt werden. 


\subsection{Einige empirische Ergebnisse von Ertragsratenberechnungen}

Seit 1973 beherrscht vor allem ein Ökonom die Literatur der Ertragsratenmessung von Humankapitalinvestitionen, nämlich GEORGE PSACHAROPOULOS, der sich sowohl methodisch als auch empirisch mit dieser Thematik auseinandersetzte. ${ }^{55}$ Obwohl die Gültigkeit seiner Ertragsratenmuster seit einiger Zeit in Frage gestellt wird, werden sie nach wie vor stetig in der bildungsökonomischen Literatur zitiert. Um die Kritik an diesem Vorgehen nachvollziehen zu können und einen kurzen Überblick über die Ertragsratenentwicklung zu bekommen, werden seine aktuellsten Berechnungen für Ertragsraten in der folgenden Tabelle dargestellt. Die letzte Aktualisierung seiner Ertragsraten wurde 1994 vorgenommen, sie liegen mittlerweile für mehr als 100 Länder vor. ${ }^{56}$

Tabelle 2.1: Ertragsraten von Bildungsinvestitionen, regionaler Durchschnitt

\begin{tabular}{l|ccc|ccc}
\multirow{2}{*}{ Region } & \multicolumn{3}{|c|}{ Soziale Ertragsrate } & \multicolumn{3}{c}{ Private Ertragsrate } \\
& Primary & Sec. & Higher & Primary & Sec. & Higher \\
\hline Afrika südlich der Sahara & 24,3 & 18,2 & 11,2 & 41,1 & 26,6 & 27,8 \\
Asien & 19,9 & 13,3 & 11,7 & 39,0 & 18,9 & 19,9 \\
Europa/mittlerer Osten/Nordafrika & 15,5 & 11,2 & 10,6 & 17,4 & 15,9 & 21,7 \\
Latein Amerika/Karibik & 17,9 & 12,8 & 12,3 & 26,2 & 16,8 & 19,7 \\
OECD & 14,4 & 10,2 & 8,7 & 21,7 & 12,4 & 12,3 \\
Welt & 18,4 & 13,1 & 10,9 & 29,1 & 18,1 & 20,3 \\
\hline
\end{tabular}

Die Daten für die nicht-OECD-Länder beziehen sich hauptsächlich auf die späten 70er Jahre. Quelle:Psacharopoulos (1994), S. 1328.

Die Tabelle zeigt, dass sich das Muster der Ertragsraten auch durch die Einbeziehung der neuen Daten nicht gewandelt hat: Erstens sind die Ertragsraten in der Primarausbildung am höchsten, gefolgt von der Sekundar- und dann der Universitätsausbildung.

Zweitens setzt sich diese abnehmende Ertragsrate der zusätzlichen Bildung auch im Ländervergleich fort. Je entwickelter ein Land ist, desto niedriger sind die Ertragsraten auf allen Ausbildungsstufen. ${ }^{57}$

Diese beiden Aussagen zusammen implizieren, dass die Rangfolge der Ertragsraten für die unterschiedlichen Bildungsstufen stabil bleibt, während das Niveau

55 Psacharopoulos (1973), Psacharopoulos (1985), S. 583-597, Psacharopoulos (1994), S. 1325-1343.

56 Die hier zitierten Ertragsraten beziehen sich auf die Alter-Einkommens-Profil Methode. Psacharopoulos stellt in seiner Veröffentlichung von 1994 auch die Ertragsraten nach der Methode nach Mincer vor, die in den meisten Fällen nicht zu abweichenden Ergebnissen führt. Vgl. Psacharopoulos (1994), S. $1342-1343$.

57 Moonwon Kang gibt hierzu zu bedenken, dass der Konsumnutzen der Bildung in entwickelten Ländern höher ist als in Entwicklungsländern und daher die pekuniären Ertragsraten in höher entwickelten Ländern stärker unterbewertet ist. Vgl. Moonwon Kang (1993), S, 32-36. 
der Ertragsraten mit zunehmendem Entwicklungsstand sinkt. Insbesondere die Aussage über die stabil bleibende Rangfolge gilt heute für einige Länder als widerlegt.

Drittens sind die privaten Ertragsraten auf allen Ausbildungsstufen höher als die sozialen, was auf die staatliche Subventionierung der Ausbildung in den meisten Ländern zurückzuführen ist. Die Diskrepanz zwischen der privaten und der sozialen Ertragsrate ist bei der Universitätsausbildung am stärksten ausgeprägt, wodurch die Frage nach der verteilungspolitischen Problematik einer (hohen) Subventionierung der Hochschulausbildung aufgeworfen wird. 58

Viertens sinken die Ertragsraten im Länderdurchschnitt mit der Zeit. Im Verlauf von 15 Jahren sind alle Ertragsraten um 2 bis 8 Prozentpunkte gesunken. Interessant ist hier, dass die private Ertragsrate der höheren Ausbildung von diesem Muster abweicht und sogar um 1,7 Prozentpunkte gestiegen ist. Diese Entwicklung zeigt nicht nur, dass die relative Position der höheren Ausbildung im privaten Kalkül gestiegen ist, sondern auch, dass sie bei der Verteilung der Subventionen ihre Position noch verbessern konnte. 59

Obwohl die Männer meistens höhere Löhne beziehen als die Frauen, sind die Ertragsraten für Frauen häufig höher als für Männer. Diese fünfte Erkenntnis kann dadurch erklärt werden, dass die Opportunitätskosten der Ausbildung für Frauen häufig niedriger sind als für Männer, und dass die Frauen erst durch die Ausbildung in der Lage sind, am Arbeitsmarkt zu partizipieren.

Diese Ertragsratenmuster wurden während der letzten 20 Jahre regelmäßig publiziert und fanden Eingang in die bildungsökonomische Diskussion. In neuerer Zeit wurden sie allerdings für einzelne Länder empirische widerlegt, was die Frage nach ihrer Relevanz in der Bildungspolitik aufwarf.

BENNELL kritisiert zum Beispiel die Aggregation von den Ertragsraten verschiedener Länder zu allgemeingültigen Mustern. Weil sich die Methoden der einzelnen Autoren, die Datenqualität, die wirtschaftlichen und geografischen Umstände der Länder stark voneinander unterscheiden, hält er die Bildung von Länderdurchschnitten für nicht zulässig. Außerdem zeigt er, dass die Unterschiede in den Ertragsraten zwischen einigen Ländern Afrikas südlich der Sahara so groß sind, dass die Durchschnittsbildung jeden Aussagewert verliert. Ein Vergleich der Daten auf Länderniveau zeigt, dass das von PSACHAROPOULOS ermittelte Ertragsratenmuster für einige Länder nicht gilt. Er kritisiert, dass diese Muster

58 Wenn die Ausbildung aus allgemeinen Steuermitteln finanziert wird, stellt sich die Frage, wer tatsächlich zahlt und wer letztendlich von den Bildungsausgaben profitiert. Werden die Hochschulen vor allem von Kindern reicherer Familien besucht, ist die besonders hohe Subventionierung dort verteilungspolitisch höchst brisant. Vgl. Psacharopoulos (1996), S. 341. 
dennoch die Aktivitäten der Weltbank in dieser Region beeinflusst haben. In seinen Schlussfolgerungen fordert er deshalb, dass die einfache Aggregation von Länderdaten aufgegeben wird. 60 Obwohl er zeigt, dass die sozialen Ertragsraten in den letzten 20 Jahren in fast allen Ländern deutlich gesunken sind, weist er ausdrücklich darauf hin, dass dies nicht heißen kann, dass öffentliche Investitionen in Ausbildung an Priorität verlieren sollte. Vielmehr müsse unbedingt die Qualität der Ausbildung in der Region verbessert werden. „...given the well-established (direct and indirect) productivity enhancing effects of education, in particular at the primary and lower secondary levels, potential social ROREs remain as high as ever."61

Auch SIPHAMBE ermittelte für Bostwana abweichende Muster für die privaten Ertragsraten. ${ }^{62}$ Die Ertragsrate ist am höchsten für die höhere Sekundarbildung (185\%) gefolgt von der Ertragsrate der unteren Sekundarbildung (84\%) und am niedrigsten für die Primarbildung (7\%). Verglichen mit den Ertragsraten einer Studie der USAID von 1984 sind seine Ergebnisse (insbesondere die der Primaria und der unteren Sekundaria) sehr niedrig. Diese Abnahme der Ertragsraten über die Zeit geht konform mit dem von PSACHAROPOULOS ermittelten Muster. Allerdings zeigt sich auch hier, dass insbesondere die niedrigeren Ausbildungsstufen im privaten Kalkül an Relevanz verloren haben.

KNIGHT/SABOT/HOVEY liefern eine Erklärung für diesen Verfall der Ertragsraten. Viele Entwicklungsländer haben in den letzten Jahren ihre Einschulungsquoten drastisch erhöhen können, während die Beschäftigung im Lohnsektor wesentlich niedrigere Wachstumsraten verzeichnet hat. Vor zwei oder drei Dekaden war das Angebot an Arbeitskräften mit abgeschlossener Primarausbildung noch niedrig in Relation zu den vorhanden Arbeitsplätzen. Ein Primarschulabschluss war die Eintrittskarte für einen gut bezahlten Job.63 Je knapper die ausgebildeten Arbeitskräfte sind, desto größer sind die individuellen Erträge der Ausbildung.64 Mit der extremen Ausweitung des Ausbildungssystems wurde die Knappheit beseitigt. Die gleichen Arbeitsplätze konnten mit Sekundarschulabsolventen besetzt werden, was zu einem Verlust der Relevanz der Primarausbildung für den Arbeitsmarkt führte.

63 Vgl. Knight/Sabot/Hovey (1992), S. 194. Hier wird deutlich, dass die in der Vergangenheit ermittelten hohen Ertragsraten nur für eine marginale Anzahl von Individuen gelten konnten. Mit der umfassenden Ausweitung der Ausbildung verloren sie ihre Aussagekraft. Vgl. dazu den Abschnitt 2.1.4 Ertragsratenarten. 
Die Autoren kritisieren an den herkömmlichen Ertragsraten, dass sie auf AltersEinkommens-Profilen basieren, die die durchschnittlichen Einkommenserfahrungen von Absolventen einer Bildungsstufe abbilden, unabhängig von dem Zeitpunkt, zu dem sie den Arbeitsmarkt betreten haben. Sie ermitteln anhand kenianischer Daten von 1980 Ertragsarten für zwei verschiedene Kohorten. Die „neue“ Kohorte beinhaltet dabei nur Lohnempfänger, die ab 1975 den Arbeitsmarkt betreten haben. Die erfahrene Kohorte beinhaltet alle anderen Lohnempfänger, die also seit 6 oder mehr Jahren am Arbeitsmarkt sind.

Anhand dieser Daten zeigen sie, dass die Ertragsrate der Primarbildung stark von der gewählten Kohorte abhängt, während die Rate für die Sekundarbildung relativ stabil bleibt.

Tabelle 2.2: Soziale Ertragsraten in Kenia, 1980

\begin{tabular}{l|ccc|} 
& Alle Kohorten & erfahrene Kohorte & neue Kohorte \\
\hline Primaria & 17 & 19 & 12 \\
Sekundaria & 13 & 14 & 13 \\
\hline
\end{tabular}

Quelle: Knight/Sabot/Hovey (1992), S. 202.

Während die Ertragsraten für die gesamte Stichprobe und die erfahrene Kohorte dem von PSACHAROPOULOS ermittelten Muster entsprechen, dreht sich die Rangfolge für die jüngere Kohorte um. Die Autoren zeigten damit eindrucksvoll, dass sich die Hierarchie der Ertragsraten mit der Entwicklung eines Landes ändern kann. Diese Erkenntnis sollte bei der Ermittlung von Ertragsraten berücksichtigt werden. ${ }^{65}$

GONZÁLEZ/MILES untersuchen die Reallohnentwicklung für Arbeiter in Uruguay und zeigen, dass die Erträge der universitären Ausbildung von 1986 bis 1997 signifikant gestiegen sind, während die Erträge der Primarbildung im gleichen Zeitraum sanken. 66

GLEWWE ermittelte für Ghana, dass ein zusätzliches Schuljahr keinen Einfluss auf die Löhne im privaten Sektor hat. Er gibt als eine mögliche Erklärung die schlechte und ungleichmäßige Qualität der ghanaischen Ausbildung an. Die Anzahl der Schuljahre ist damit kein guter Indikator für Humankapital. Werden sie dennoch zur Ermittlung von Ertragsraten herangezogen, sind die Ergebnisse verzerrt. Einige Erweiterungen des Modells zeigen, dass die in der Schule erlernten Kenntnisse und Fähigkeiten die Lohndifferenzen im privaten Sektor stärker beeinflussen als die Anzahl der absolvierten Schuljahre. Er schließt daraus, dass die herkömmlichen Ertragsraten für Entscheidungen in der Bildungspolitik kaum Relevanz haben. ${ }^{67}$

\footnotetext{
65 Vgl. Knight/Sabot/Hovey (1992), S. 192-205.

66 Vgl. González/Miles (2001), S. 145.

67 Vgl. Glewwe (1996), S. 267-290.
} 
Schaut man sich die Ertragsraten für Peru an, die in die Berechnungen von PSACHAROPOULOS eingegangen sind, wird die Forderung nach einer Betrachtung der einzelnen Länderdaten unterstützt. Leider liegen nur Daten für die privaten Ertragsraten von 1990 vor. Aber auch hier wird das Muster widerlegt, weil die höhere Bildung die höchsten Ertragsraten aufweist (40\%) gefolgt von der Primaria (13,2\%) und der Sekundaria (6,6\%). ${ }^{68}$ Auch in Peru scheint die Basisausbildung in Relation zur höheren Bildung an Relevanz verloren zu haben.

Auch die Autoren des IDB Report 1998-1999 haben sich der Entwicklung der Ertragsraten angenommen und neue Durchschnittsraten für Lateinamerika ermittelt. Während in den 80er Jahren die Primarbildung noch eine durchschnittliche Rate von 26\% aufweisen konnte, wurde für die 90er Jahre nur noch eine Rate von $10 \%$ ermittelt, dicht gefolgt von der Sekundaria (11\%). Die höhere Bildung weist mittlerweile mit 18\% die höchste Rate auf. 69 Im IDB Report wird daraus die folgende Schlussfolgerung gezogen: „However, the structure of returns on education in Latin America is conducive to the stratification of education, as it provides little incentive to advance in basic education unless there is the scope to have access to a university education. The small proportion of the population that reaches secondary education in Latin America is consistent with this interpretation."70 Die niedrigen Erträge für die Basisausbildung werden auf den Einfluss der Globalisierung zurückgeführt. Zum einen drängen mehr schlecht ausgebildete Arbeitskräfte aus anderen Entwicklungsländern auf den Markt, zum anderen hat der technologische Wandel die Nachfrage auf höher ausgebildete Kräfte verschoben. Die Autoren weisen aber ausdrücklich darauf hin, dass es sich bei den ermittelten Ertragsraten um private Raten handelt. Obwohl die Basisausbildung für den individuellen Investor geringe Erträge bietet, ist sie gesellschaftlich viel höher zu bewerten. Sie bietet Basis für weitere Nutzen, wie zum Beispiel die Verbesserung des Gesundheitsstandes, die Reduzierung der Geburtenrate und die Erhöhung des Lebensstandards. ${ }^{71}$ Zur gesellschaftlichen Bewertung der Nachfrage müssten also soziale Ertragsraten ermittelt werden.

69 Vgl. IDB (1998), S. 51. Hier werden auch Ertragsraten für die 90 er Jahre für Peru ermittelt, die allerdings von denen bei Psacharopoulos abweichen und für alle drei Bildungsstufen um die 15\% liegen.

70 IDB (1998), S. 52.

71 Vgl. IDB (1998), S. 53 und die Ausführungen zu den Effekten der Ausbildung in Abschnitt 2.1.2. 


\subsection{Fazit und Anwendbarkeit des Ertragsratenansatzes}

Die empirischen Arbeiten zeigen, dass in der Vergangenheit die Betonung der Elementarbildung in Entwicklungsländern eine gewisse Berechtigung hatte. Die hohen Ertragsraten signalisierten sogar bei Betrachtung ausschließlich der direkten monetären Erträge den hohen Wert einer Investition in diese Ausbildung. Allerdings zeigen die Daten auch, dass mit dem Erreichen einer umfassenden Grundausbildung und den veränderten Umweltbedingungen die Basisausbildung im privaten Kalkül ihren Rang verlieren kann. Der soziale Wert der Basisausbildung wird allerdings nicht angezweifelt, da diese Ausbildung den Grundstein für jede weitere Ausbildung darstellt und damit Voraussetzung für weitere Nutzensteigerungen bietet. Ertragsraten sollten allerdings immer nur für einzelne Länder berechnet und vor dem Hintergrund ihrer ökonomischen, sozialen und geografischen Lage interpretiert werden.

In dieser Arbeit soll das Bildungsverhalten der Individuen genauer untersucht werden. Dazu wird angenommen, dass einer Bildungsentscheidung auch immer eine (unter Umständen auch unterbewusste) Abwägung der Kosten und Nutzen vorausgeht. Obwohl die Bildungsentscheidungen der Bevölkerung natürlich nicht auf derartig ausgefeilten Berechnungen basieren ${ }^{72}$, wie sie in diesem Kapitel dargestellt wurden, bietet der Ertragsratenansatz ein sinnvolles Instrumentarium zur Formalisierung des angenommenen subjektiven Kalküls.

Bei einer derartigen Verwendung des Ansatzes greifen auch die genannten Kritikpunkte nicht. Erstens soll der Ansatz nicht zu einer Bewertung von Ausbildung führen, sondern er soll zur Erklärung des Entscheidungsprozesses beitragen. Deshalb mindert die Kritik, die sich hauptsächlich gegen die exakte Bewertung von Bildungsinvestitionen richtet, den Wert des Ansatzes für die Untersuchung nicht. Vielmehr sind gerade die subjektiven Wertschätzungen und die individuellen Erwartungen Gegenstand der Untersuchung. Sie werden als solche dargestellt und keiner Bewertung unterzogen.

Zweitens ist es für die individuelle Entscheidung irrelevant, ob die erwarteten Erträge tatsächlich auf Produktivitätsverbesserungen oder nur auf der Signalwirkung der Ausbildung beruhen.

Drittens sind es bei der Elementarbildung vor allem die Eltern, die die Bildungsentscheidung treffen. Mit dem Haushalt als Entscheidungseinheit werden die

72 Zum einen haben die potentiellen Investoren nicht die gleichen Informationen zur Verfügung wie die Bildungsökonomen. Zum anderen wird das Individuum die Ausbildung nicht nur als Investition in höhere Einkommen betrachten. Viel mehr als präzise Berechnungen, werden individuelle Erfahrungen und Erwartungen den Wert der Ausbildung für das Individuum bestimmen. Vgl. Blaug (1991), S. 1. 
Effekte, die die Familie betreffen internalisiert. Externe Effekte spielen bei der privaten Bildungsentscheidung keine Rolle und werfen damit auch keine Zuordnungs- oder Bewertungsprobleme auf.

Der Ertragsratenansatz stellt damit eine adäquate Grundlage für die weiteren Überlegungen dar. 


\section{Die optimale Bildungsentscheidung}

Die Ertragsraten können nicht nur als statistische Zusammenfassung der Kosten und Erträge von Ausbildung betrachtet werden. Sie können dem Individuum auch als Variable bei der Bildungsentscheidung dienen. In einem perfekten Wettbewerbsmodell wird Allokationseffizienz erreicht, wenn die Grenzerträge aller alternativer Investitionen gleich sind. Das heißt, wenn der Ertrag einer Investition $A$ höher ist als der Ertrag der Investitionsalternative $B$, sind Effizienzsteigerungen möglich, indem Ressourcen von B nach A umgeleitet werden. Für jedes Investitionsobjekt, müssen die Grenzkosten den Grenzerträgen entsprechen, um Effizienz zu erreichen. Welche Auswirkungen dieses Verhalten auf die Bildungsentscheidung hat, soll anhand eines grafischen Modells von G.S. BECKER aufgezeigt werden.

\subsection{Das Grundmodell}

BECKER $^{73}$ hat ein theoretisches Modell der Bildungsinvestitionen entwickelt, nach dem der Nutzen eines Individuums bei einem gewissen Bildungsstand maximiert werden kann. Dabei geht er von rationalem Investitionsverhalten der Individuen aus. Anhand dieses Modells kann gezeigt werden, dass Individuen, deren Ertragsraten und Finanzierungsbedingungen voneinander abweichen, auch unterschiedliche Bildungsmengen nachfragen werden.

\subsubsection{Grafische Darstellung}

Das Optimierungskalkül soll anhand der Grafik 3.1 veranschaulicht werden. An der Ordinate sind die Ertragsrate der Bildung und der Marktzins abgetragen; und an der Abzisse sind die Bildungsinvestitionen abgetragen. Die Bildungsinvestitionen werden in physischen Einheiten (Schuljahre oder Bildungsstufen) gemessen. ${ }^{74}$

73 Das Humankapitalmodell von Becker verfolgt den Investitionsansatz und geht von individuellem Kalkül aus. Das Quantity-Quality-Modell von Becker/Lewis betrachtet Bildung auch als Konsumgut und betrachtet die Familie als Entscheidungseinheit. Wie die Ausführungen im 5. Kapitel zeigen, werden diese theoretischen Ansätze und ein parallel entwickeltes Modell von Ben-Porath noch immer als Grundlage für die empirische Untersuchung von Bildungsnachfragefunktionen herangezogen. Vgl. Becker (1983), Becker/Lewis (1973), S. S279-S288, Becker/Tomes (1976), S. S143-S162 und BenPorath (1967).

74 Hier ergibt sich eine Abweichung vom Becker-Modell. Da Becker anhand des Modells die personelle Einkommensverteilung erklären möchte, misst er die Bildungsniveaus anhand der investierten Geldbeträge. Vgl. Becker (1983), S. 97. Der Zusammenhang zur nachgefragten Bildungsmenge wird aber deutlicher, wenn direkt die Bildungsniveaus abgetragen werden. Vgl. dazu auch Psacharopoulos (1973), S. 77-79. 
Die Ertragsratenkurve stellt die Grenzerträge der Ausbildung für ein Individuum dar. Sie werden durch die Ertragsraten der jeweiligen Ausbildungseinheiten angegeben. Die steigende Kurve zeigt die marginalen Finanzierungskosten für die jeweiligen Bildungsniveaus und wird der Einfachheit halber anhand des Zinssatzes gemessen. ${ }^{75}$ Solange die Ertragsrate der Ausbildung über der Zinsrate zu deren Finanzierung liegt, kann das Einkommen gesteigert werden, indem zusätzlich in Bildung investiert wird. Ein Bildungsniveau, dessen Ertragsrate unter den Zinskosten liegt, ist ebenfalls nicht effizient. Demzufolge kann das Einkommen maximiert werden, wenn bis zu dem Punkt investiert wird, bei dem sich die beiden Raten ausgleichen. Für das betrachtete Individuum ergibt sich also eine optimale Investitionshöhe von $S^{*}$ Schuljahren.

\section{Grafik 3.1: Die optimale individuelle Investitionsentscheidung}

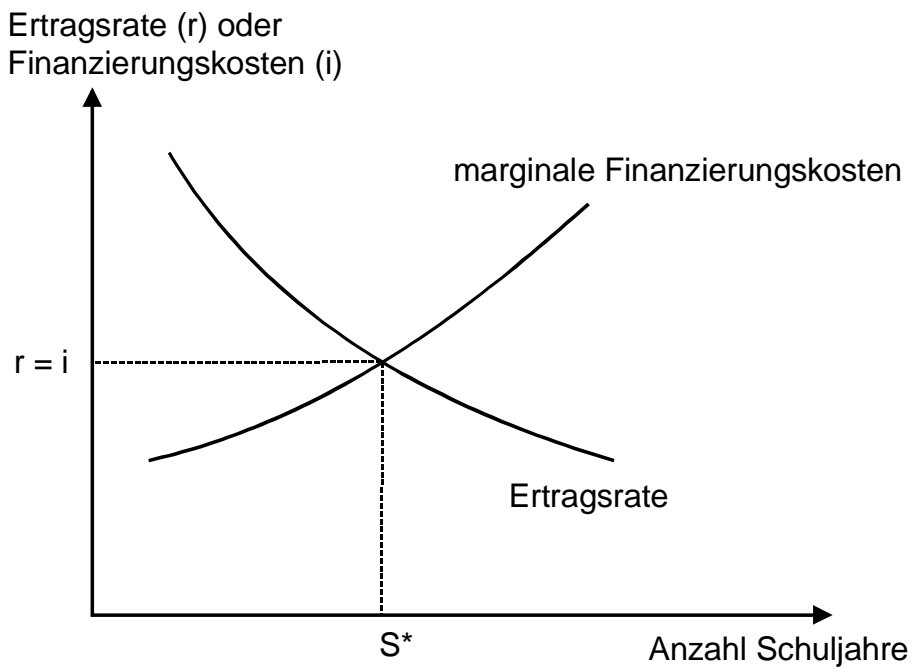

Quelle: Psacharopoulos (1973), S. 77.

Für die Kurve der Grenzerträge ${ }^{76}$ wird aufgrund der folgenden Überlegungen ein fallender Verlauf angenommen. Anders als bei Sachkapital ist das Humankapital an die investierende Person gebunden. Wenn die physische und mentale Aufnahmekapazität der investierenden Person begrenzt ist, sinken die Erträge jeder zusätzlich investierten Einheit. Weiterhin wird jede zusätzliche Einheit in späteren Zeitperioden investiert, womit bei begrenzter Lebenszeit die Ertragszeiten verkürzt werden. Außerdem steigen mit dem akkumulierten Humankapital die Opportunitätskosten der Bildung an, was die Ertragsrate schmälert. Wie die obigen Ausführungen zeigten, ist dieses auch von PSACHAROPOULOS

\footnotetext{
75 Becker nennt diese Kurven Nachfrage- und Angebotsfunktionen. Da es sich aber nicht um das konventionelle Konzept von Angebot und Nachfrage handelt, führt diese Terminologie zu Verwirrungen.

76 Vgl. Becker (1983), S. 97-102. Becker bezeichnet die Kurve der Ertragsraten als Nachfragekurve des Individuums nach Bildung. Diese Nachfragefunktion darf nicht mit dem konventionellen Konzept der Bildungsnachfrage verwechselt werden, welche eine positive Funktion der Ertragsrate ist. Um Verwirrungen zu vermeiden, soll diese Bezeichnung hier vermieden werden.
} 
ermittelte Ertragsratenmuster nicht mehr für alle Länder nachzuweisen. Dennoch soll im folgenden die optimale Bildungsentscheidung unter der Annahme sinkender Grenzerträge dargestellt werden, da die theoretischen Modelle auf dieser Annahme basieren und die meisten empirischen Untersuchungen diese Modelle verwenden.

Der steigende Verlauf der Kostenkurve kommt durch die starke Segmentierung des Kapitalmarktes für Bildungsinvestitionen zustande. Zum Beispiel werden zunächst Subventionen oder Unterstützung durch die Eltern, subventionierte Kredite und eigene Mittel zur Finanzierung herangezogen, bevor Kredite zum Marktzinssatz herangezogen werden. Je segmentierter der Kapitalmarkt desto steiler verläuft die Angebotskurve.

Die Investitionsentscheidung ist optimal, wenn der Grenzertrag den marginalen Zinskosten entspricht. Der Schnittpunkt der beiden Kurven determiniert die Investitionshöhe und die Ertragsrate der Investition. Dieses Modell impliziert, dass unterschiedliche Bildungsinvestitionen bei Individuen durch deren Ertragsratenoder Finanzierungskurven verursacht werden: Personen mit besonders hohen Ertragskurven oder besonders niedrigen Finanzierungskurven ermitteln höhere optimale Bildungsinvestitionen als andere. Je ungleicher die Lage der Kurven angenommen wird, desto größer sind die Unterschiede in der Bildungsnachfrage zu erwarten. Wie es zu diesen ungleichen Lagen der Kurven kommen kann, wird im folgenden Abschnitt gezeigt.

\subsubsection{Die Lage der Kurven}

Wie ist die Ertragsratenkurve zu interpretieren? BECKER nennt sie auch „Fähigkeitenkurve“, weil sie zum Ausdruck bringt, in welchem Maß das Individuum in der Lage ist, von der Ausbildung zu profitieren. Personen, die bei gegebener Investitionsmenge höhere Ertragsraten realisieren, weisen höhere Kurvenverläufe auf. Da BECKER die Bildungsinvestitionen anhand der investierten Geldbeträge misst, argumentiert er, dass fähigere Personen in der Lage sind, gegebene Geldbeträge in größere Mengen Humankapital umzuwandeln. ${ }^{77}$ Werden (wie hier) die Investitionen in Bildungseinheiten gemessen, werden Personen als besonders fähig bezeichnet, wenn sie in der Lage sind, bei gegebenem Bildungsniveau höhere Ertragsraten zu realisieren als andere. Im zweiten Kapitel wurde aufgezeigt, welche Faktoren die Ertragsraten eines Individuums beeinflussen können. Wer in der Lage ist, ein gegebenes Bildungsniveau besser in höhere Einkommen umzusetzen, oder dieses Bildungsniveau zu geringeren

77 Die Aussagen Beckers ändern sich nicht, wenn die Investitionen in Bildungsniveaus gemessen werden. 
Kosten oder mit geringerem Zeitaufwand 78 zu erreichen, wird eine höhere Kurve realisieren als die Vergleichsperson. Diese Fähigkeiten können angeboren, das Ergebnis vorteilhafter Familienverhältnisse ${ }^{79}$ oder das Resultat komplementärer Investitionen sein.

Die Lage der Finanzierungskurve wird ebenfalls zwischen den Individuen variieren. Sie wird vor allem durch die finanzielle Situation des Individuums festgelegt. BECKER bezeichnet die Kurve daher auch als „Möglichkeitenkurve“. Sie liegt für diejenigen am niedrigsten, die leichter Zugang zu finanziellen Mitteln haben. Ihre Kurve verlagert sich nach unten, da dieselbe Investitionshöhe geringere Kosten verursacht. Die gleiche Argumentation, die den steigenden Verlauf der Kurve erklärt - nämlich die Segmentierung am Kapitalmarkt - führt auch zu den unterschiedlichen Kredit-Konditionen verschiedener Individuen. Eine Vielzahl von Gründen kann dazu führen, dass einige besseren Zugang zu Stipendien, staatlichen Subventionen oder günstigen Krediten bekommen. Hier kann u. a. der Wohnort oder Beziehungen oder einfach Glück eine Rolle spielen. Vor allem aber ein wohlhabendes und großzügiges Elternhaus erleichtert die Finanzierung von Ausbildungsinvestitionen.

Darüber hinaus kann der Marktzins auch als Ertragsrate einer alternativen Investition betrachtet werden. Für Personen, deren Entscheidung darin besteht, vorhandene Mittel in Humankapital zu investieren oder sie am Kapitalmarkt anzulegen, ist diese Interpretation relevant. ${ }^{80}$

Der wichtigste Grund für die Lage und den Verlauf der Möglichkeitenkurve liegt also im Zugang zu Kapital. Der Verlauf und die Lage der Fähigkeitenkurve wird durch das Individuum selbst und dessen sozioökonomisches Umfeld bestimmt. Die Faktoren, die die Kurven beeinflussen sind nicht eindeutig zu trennen. Zum Beispiel kann sich die Diskriminierung einer Bevölkerungsgruppe sowohl auf die Finanzierung als auch auf die Umsetzung der Bildung in Einkommen auswirken. Die Bezeichnung des Ertragsratenverlaufs als „Fähigkeitenkurve“ ist damit nicht ganz glücklich gewählt, da hier auch äußere Einflüsse eine Rolle spielen. Deshalb wird im folgenden die Bezeichnung Ertragsratenkurve und Finanzierungskostenkurve beibehalten. Zur genaueren Analyse von Nachfrageunterschieden müssen die Effekte einzeln untersucht werden.

78 Ein geringerer Zeitaufwand kann zu niedrigeren Opportunitätskosten führen, da die verbleibende Zeit anderen Beschäftigungen gewidmet werden kann. Ein Schüler, der seinen Abschluss sehr früh erreicht, braucht kein weiteres Jahr auf eigenes Einkommen zu verzichten. Die Kosten durch den Verzicht auf eine alternative Zeitverwendung gehen in die Ermittlung der Ertragsraten ein. 


\subsection{Individuelle Kurvenverläufe}

Wenn nur die Finanzbedingungen zwischen den Individuen variieren würden und nur eine einzige Grenzertragskurve für alle Individuen gleichermaßen gälte, wäre jeder Unterschied in der Bildungsnachfrage durch die Finanzbedingungen erklärbar. ${ }^{81}$ Je besser die Finanzbedingungen (d.h. je niedriger die Kurve) desto mehr Bildung würde nachgefragt. Wäre auf der anderen Seite nur die Ertragsratenfunktion variabel bei einer allgemeingültigen Finanzierungskostenkurve für alle Individuen, hätten allein die Ertragsraten Einfluss auf die Bildungsinvestitionen. ${ }^{82}$ Menschen, die niedrigere Ertragsraten ihrer Ausbildung erwarten, würden weniger in ihre Ausbildung investieren.

Beide Fälle sind als irreale Extremfälle zu bezeichnen. Vielmehr werden zwischen den Individuen sowohl die Ertragsraten als auch die Finanzsituationen variieren. Die folgende Grafik 4 zeigt eine Auswahl von Ertrags- und Finanzierungskurven.

\section{Grafik 3.2: Variierende Ertragsraten und Finanzbedingungen}

Marginale Erträge und Finanzierungskosten

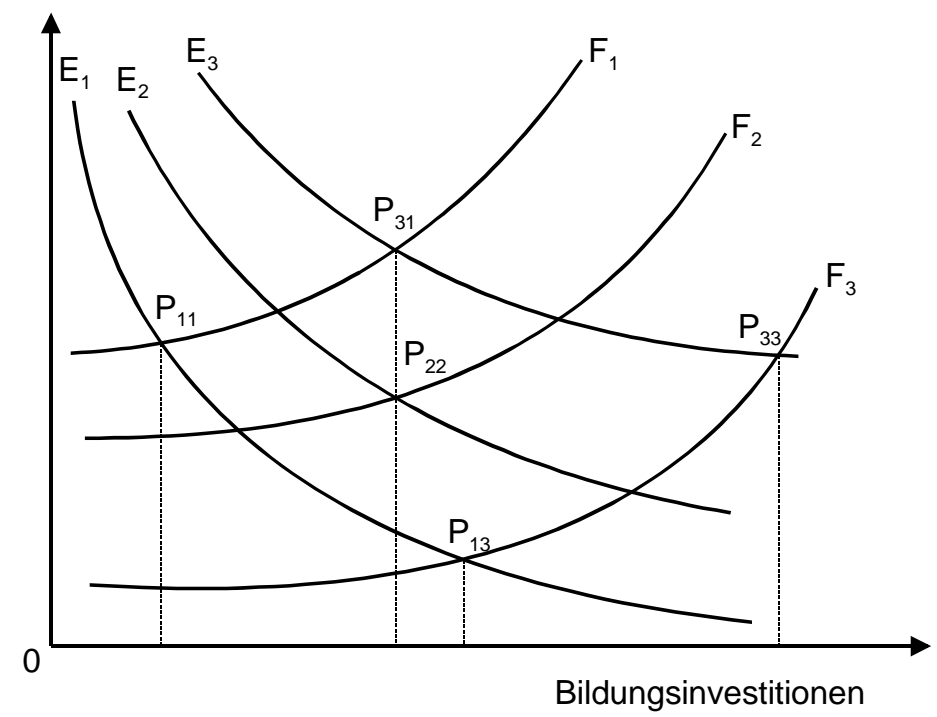

Quelle: Becker (1983), S. 116.

Wenn die beiden Kurven für verschiedene Individuen völlig unabhängig voneinander verlaufen (sie also unkorreliert sind), ist jeder Schnittpunkt in der Grafik ein möglicher Investitionspunkt. Sind alle Kombinationen von Kurven möglich, kann aus dem bekannten Verlauf einer Kurve nicht mehr auf die optimalen Bil-

81 Becker nennt diesen Spezialfall „Egalitarian Approach“. In diesem Fall müssten alle beobachtbaren Investitionspunkte auf der für alle gleichen Ertragsratenkurve liegen. Vgl. Becker (1983), S. 106-109. 
dungsinvestitionen geschlossen werden. Ungünstige Finanzbedingungen könnten die Vorteile hoher Ertragsraten konterkarrieren - und umgekehrt. Ist aber die Lage einer Kurve nicht unabhängig von der Lage der anderen Kurve, lassen sich wieder Investitionsmuster herleiten. Dies wird im folgenden gezeigt.

Realistischerweise kann angenommen werden, dass die Ertragsraten- und Finanzierungsbedingungen positiv korreliert ${ }^{83}$ sind. Das heißt, es wird angenommen, dass hohe Ertragsratenkurven mit niedrigen Finanzierungskurven gepaart sind. Eine Person, die leichter Zugang zu Mitteln zur Finanzierung ihrer Ausbildung hat, besitzt auch höhere Fähigkeiten, von dieser Ausbildung zu profitieren. ${ }^{84}$ Anders ausgedrückt: Die Vorteile hoher Ertragsraten werden noch durch günstige Finanzbedingungen verstärkt. Ebenso werden Personen mit niedrigen Ertragsraten noch durch hohe Finanzierungskosten belastet, was ihr optimales Bildungsniveau noch zusätzlich senkt. Individuen mit extremen Kurvenverläufen weisen auch extrem niedrige bzw. extrem hohe optimale Bildungsniveaus auf. In der Grafik ergeben sich die Punkte $P_{11}, P_{22}$ und $P_{33}$ als optimale Investitionspunkte dreier Individuen mit unterschiedlichen Möglichkeiten und Fähigkeiten. Wird also eine positive Korrelation zwischen den finanziellen Möglichkeiten und den Ertragsraten (Fähigkeiten) der Individuen angenommen, ist zu erwarten, das die Bildungsnachfrage stark variiert. Allerdings kommt es zu relativ ausgeglichenen Ertragsraten bei den verschiedenen Bildungsniveaus.

Eine negative Korrelation ${ }^{85}$ der Möglichkeiten und er Fähigkeiten bedeutet, dass hohe Ertragsratenkurven mit hohen Finanzierungskurven gepaart sind. Dabei wird angenommen, dass eine Person mit besonders hohen Fähigkeiten von der Ausbildung zu profitieren, besonders hohe (ungünstige) Finanzierungskosten zu tragen hat. Die Vorteile der hohen Ertragsraten werden also durch ungünstige Finanzbedingungen konterkarriert. Ebenso wird der Nachteil besonders niedriger Ertragsraten durch eine günstige Finanzsituation abgeschwächt. $\mathrm{Da}$ sich hier die beiden Bedingungen entgegengesetzt auswirken, entstehen keine extremen Unterschiede in den optimalen Bildungsniveaus.

In der Grafik ergeben sich die Gleichgewichtspunkte dreier Individuen mit unterschiedlichen Finanz- und Ertragsratensituationen bei $P_{31}, P_{22}$ und $P_{13}$. Hier zeigt sich, dass die Person, die die niedrigsten Erträge aus ihrer Ausbildung

83 Die positive Korrelation bezieht sich auf die Bedingungen für die Individuen. Daraus folgt, dass die eine Kurve besonders hoch und die andere besonders niedrig verläuft. Dies sollte berücksichtigt werden, um Verwirrungen bei der Verwendung des Begriffes bei der Kurveninterpretation zu vermeiden. 
erzielt, das höchste optimale Bildungsniveau aufzeigt. Die Nachteile aus den geringen Fähigkeiten können also durch die guten Finanzierungsmöglichkeiten überkompensiert werden. Wie die Grafik weiterhin zeigt, ist es im Fall eines negativen Zusammenhangs möglich, dass Personen mit dem gleichen Bildungsniveau unterschiedliche Ertragsraten realisieren. Werden bei gleichen Bildungsniveaus also unterschiedliche Ertragsraten beobachtet, müssen die Kurven dieser Personen negativ korreliert sein, unabhängig davon, wie der generelle Zusammenhang zwischen den Kurven verläuft.

Eine negative Korrelation der Kurven führt also zu geringeren Unterschieden in den Investitionshöhen, sie ermöglicht aber auch Ertragsratendifferenzen bei gleichen Bildungsniveaus. Aus einzelnen Beobachtungen kann weder die Situation der Betroffenen noch eine gesellschaftliche Effizienzkurve abgeleitet werden.

Zusammenfassend lässt sich also sagen, dass große Unterschiede in der Bildungsnachfrage dann zu erwarten sind, wenn sowohl die Möglichkeiten als auch die Fähigkeiten unter den Individuen stark variieren und außerdem ein positiver Zusammenhang zwischen diesen beiden Kurven besteht.

\subsection{Fazit zur Anwendung der grafischen Darstellung}

Die obigen Ausführungen haben gezeigt, dass sich für Individuen mit unterschiedlichen Kurvenverläufen verschiedene optimale Bildungsniveaus mit den dazugehörigen Ertragsraten ergeben. Je größer die Unterschiede in den Ertragsraten und Finanzierungssituationen ausfallen, desto ungleicher stellen sich die Bildungssituationen dar. Im Fall positiv korrelierter Ertrags- und Finanzsituationen drückt sich der Unterschied in Form von weit streuenden optimalen Bildungsniveaus aus. Im Fall negativ korrelierter Bedingungen liegen die optimalen Bildungsmengen zwar dicht beieinander, aber die realisierbaren Ertragsraten variieren stark.

Wenn Unterschiede zwischen armen und reichen Bevölkerungsgruppen untersucht werden sollen, ist diese Erkenntnis von Bedeutung. Sollten für die Bevölkerungsgruppen voneinander abweichende Kurvenverläufe ermittelt werden, können damit die für die Bevölkerung typische Bildungsnachfrage vorausgesagt und die Verteilung der Ausbildung in der Gesellschaft erklärt werden. Diese Erkenntnisse werden noch von großer Bedeutung sein, wenn die Ergebnisse der Haushaltsbefragung aus Kapitel 6 für Voraussagen bezüglich der Bildungsnachfrage in Kapitel 8 herangezogen werden sollen. 



\section{Die Grenzen der ökonomischen Modelle}

PATRICIA R. Plante leitete ihr Buch, The Art of Decision Making' mit dem Satz ein, dass das Erkennen aller multiplen Zusammenhänge eines Entscheidungsprozesses eher einer Kunst denn einer Wissenschaft gleicht. ${ }^{86}$ Sie räumt damit ein, dass es kaum möglich sein kann, alle Einflussfaktoren zu ermitteln und ihren Einfluss auf die Entscheidung eindeutig zu bestimmen.

Wie die obigen Ausführungen zeigen, wird dieses Problem häufig umgangen, indem die Individuen als rationale Investoren mit gegebener Präferenzstruktur betrachtet und in die Berechnungen nur die relativ gut beobachtbaren und messbaren Faktoren einbezogen werden. Auf makroökonomischer Ebene und zur Bestimmung von Kennzahlen ist dieses Vorgehen durchaus zu rechtfertigen. Wenn allerdings Verhaltensunterschiede zwischen einzelnen Gruppen der Bevölkerung beobachtet werden und diese erklärt werden sollen, greift der reduzierte Ansatz zu kurz. Vor allem wenn die ökonomische Theorie herangezogen werden soll, um Politikempfehlungen zu geben, sollte sie dem tatsächlichen Erwartungsbildungs- und Entscheidungsprozeß mehr Gewicht beimessen als der logischen Abstraktion. ${ }^{87}$ Dennoch bieten die oben vorgestellten Ansätze das Instrumentarium, um den Entscheidungsprozeß systematisch zu analysieren. Inwieweit diese Instrumente genutzt werden können, und welche Einschränkungen bzw. Erweiterungen notwendig sind, wird im folgenden Abschnitt untersucht. Dabei wird zwischen dem Erwartungsbildungsprozesses und dem Prozess der Bildungsentscheidung unterschieden.

\subsection{Der Erwartungsbildungsprozess}

Bei der Bildungsentscheidung spielen die Erwartungen der Individuen eine große Rolle. Der größte Teil des Nutzens der Ausbildung (v. a. die Einkommenssteigerungen) liegt in der Zukunft. Der tatsächliche Effekt der Ausbildung ist bei der Bildungsentscheidung noch nicht bekannt. Die Bildungsnachfrage wird daher vor allem durch Erwartungen an die Zukunft bestimmt. Ob diese Erwartungen realistisch sind oder nicht ist für diese Untersuchung irrelevant. Vielmehr ist interessant, wie sie entstehen und welche Wirkungen sie auf die Bildungsentscheidung haben. Da die erwarteten Erträge der Ausbildung eine so zentrale

86 Vgl. Plante (1987), Vorwort, S. IX. In diesem Buch handelt es sich um die Entscheidungsfindung in der Schuladministration. Die Vielschichtigkeit des Entscheidungsprozesses lässt sich aber auf die Einschulungsentscheidung übertragen.

87 Vgl. Katona (1980), S. 32. Katona kritisiert die Annahme rationaler Erwartungen indem er betont, dass die Erwartungsbildung ein psychologischer Prozess ist, und dass deshalb die Erkenntnisse der Psychologie stärker berücksichtigt werden sollten. 
Rolle in der ökonomischen Theorie über das Bildungsverhalten spielen, sollte man erwarten, dass die Ökonomen ausgiebig untersucht haben, wie junge Menschen ihre Erwartungen bilden. Aber in dieser Disziplin steht man subjektiven Daten traditionell skeptisch gegenüber; „so much so that we have generally been unwilling to collect data on expectations. Instead, the norm has been to make assumptions about expectations formation."88

Theorien über den Erwartungsbildungsprozess spielen daher in der Bildungsökonomie eine große Rolle. Früher wurde in der Ökonomie generell davon ausgegangen, dass Einkommenserwartungen durch einen adaptiven Prozess geformt wurden - also das Resultat von Erfahrungen sind. 89 Schüler haben normalerweise noch keine vollständigen Einkommenserfahrungen gesammelt, die sie auf die Zukunft übertragen könnten. Sie werden daher auf ihre Beobachtungen in der Familie, im Freundeskreis oder der Nachbarschaft zurückgreifen.

Durch die Theorie der rationalen Erwartungen wurde diese Annahme abgelöst. Den Individuen wird hier ein kognitives Verständnis der Zusammenhänge und ein darauf basierendes, bewusstes Handeln unterstellt. ${ }^{90}$ Rationale Erwartungen weichen von der Realität nur durch Zufallsfehler ab. Der Vorteil dieser Annahme ist, dass Änderungen in den Erwartungen nicht zufällig oder launenhaft eintreten, und deshalb immer erklärbar sind. Auf die Bildungsentscheidung bezogen hieße dies, dass die Schüler (oder deren Eltern) genaue Kenntnisse über den Wirtschaftsprozess haben und die Auswirkungen von politischen Maßnahmen auf die Arbeitsmarktentwicklung kennen. In Kenntnis dieser Rahmenbedingungen treffen sie die Bildungsentscheidung, die ihren Nutzen maximiert. Die Annahme unterstellt i. d. R. allen Individuen den gleichen Kenntnisstand und schließt damit Verhaltensunterschiede aufgrund von unterschiedlichen Wahrnehmungen aus.

Der Disput zwischen den beiden Theorien zur Erwartungsbildung soll hier nicht ausgeweitet werden. Beide Extreme erscheinen unrealistisch. Die Fähigkeit, alle relevanten Faktoren zu kennen und zu berücksichtigen, setzt eine so hohe Intelligenz der Individuen voraus, der sicher kein Akteur gerecht werden kann. ${ }^{91}$ Ebenso unwahrscheinlich ist die Annahme, dass vergangene Erfahrungen unreflektiert in die Zukunft projiziert werden.

88 Manski (1993), S. 43.

89 In seiner Permanent Income Hypothesis legt Freeman z.B. den größten Wert bei der Ermittlung der erwarteten Einkommen auf die letzten Erfahrungen aus der Vergangenheit. Vgl. Manski (1993), S.4344. 
Empirische Überprüfungen, ob diese Theorien eine realistische Beschreibung des tatsächlichen Verhaltens darstellen, sind nicht sehr verbreitet. Obwohl einige Studien über die Erwartungsbildung in Unternehmen existieren, sind empirische Arbeiten über Erwartungen von privaten Haushalten oder Individuen kaum zu finden. 92

Aus 30 Jahren empirischer Forschung zur Erwartungsbildung schließt KATONA, dass "most commonly our behaviour is governed by habits derived from repeated and rewarded past experience."93

KATONA hält eine Mischung der Ansätze für realistischer als die Annahme eines starren Konzeptes. Außerdem weist er darauf hin, dass Erwartungen in den Bereich der Einstellungen gehören, und damit auch eine Gefühlskomponente aufweisen. Diese Gefühlskomponente (z.B. Optimismus/Pessimismus) kann sich über ganze Bevölkerungsgruppen ausbreiten und deren Erwartungen beeinflussen, während der kognitive Anteil der Erwartungen unbestimmter und von Person zu Person unterschiedlich sein kann. ${ }^{94}$

Aufgrund dieser unterschiedlichen, möglichen Einflüsse muss die Verteilung und Streuung der Erwartungen mehr Berücksichtigung finden. Es macht durchaus einen Unterschied, ob alle Individuen den gleichen Trend erwarten, oder ob sich die durchschnittlichen Erwartungen aus sehr unterschiedlichen Einzelerwartungen ergibt. Allgemeingültige Annahmen über die Erwartungen aller Individuen in einem Modell stellen die Wirklichkeit nur unzulänglich dar. Da es mittlerweile möglich ist, Erwartungen und ihre Veränderungen empirisch zu untersuchen ${ }^{95}$, ist die Zuhilfenahme eines starren Konzeptes nicht mehr nötig.

Auch MANSKI kritisiert die übliche Annahme der Ökonomen, dass die Erwartungsbildung homogen ist, die Jugendlichen ihre Erwartungen also aufgrund der gleichen Variablen bestimmen und ihre Informationen in der selben Weise umsetzen. In den meisten ökonomischen Analysen des Bildungsverhaltens werden die Annahmen zur Erwartungsbildung nicht einmal explizit benannt oder gar in Frage gestellt. ${ }^{96}$ Dabei gibt es keinerlei Hinweis darauf, dass die gemachten Annahmen richtig sind oder eine Missspezifizierung vernachlässigbar ist. $\mathrm{Er}$ stellt ein einfaches stationäres Humankapitalmodell auf, um zu zeigen, dass für

92 Vgl. Das/Van Soest (2000), S. 1. Die Autoren nutzten Panel-Daten über erwartete und realisierte Einkommensveränderungen, um zu untersuchen, ob Haushaltsvorstände rationale Erwartungen haben. Die Rational Expectation Hypothesis mußte abgelehnt werden. Ebenso kann die Hypothese gestestet werden, dass die Erwartungen adaptiv gebildet wurden. Auch diese Hypothese hielt dem Test nicht stand. Vgl. Das/Van Soest (2000), S. 30-31.

Katona (1980), S. 33.

94 Vgl. Katona (1980), S. 33.

95 Vgl. Katona (1980), S. 32.

96 Vgl. Manski (1993), S. 44. 
alternative Annahmen über den Erwartungsbildungsprozess unterschiedliche Ergebnisse berechnet werden. Damit zeigt er, dass ohne Kenntnis der Erwartungsbildung weder das beobachtete Bildungsverhalten eindeutig interpretiert, noch die angestrebten Erträge abgeleitet werden können. ${ }^{97}$

DAS/VAN SOEST schließen aus ihrem Test der Rational Expectation Hypothesis, dass die Bildung alternativer Theorien für die Erwartungsbildung eine Herausforderung an die Zukunft darstellt. ${ }^{98}$

Bei der Untersuchung der Ertragserwartungen der verschiedenen Bevölkerungsschichten in Kapitel 6, soll daher keines der Annahmemodelle ausschließlich herangezogen werden. Bei der Interpretation der Ergebnisse sollen beide Ansätze als mögliche Einflussfaktoren berücksichtigt werden.

\subsection{Der Prozess der Bildungsentscheidung}

In diesem Abschnitt sollen die möglichen Mechanismen untersucht werden, die der individuellen Bildungsentscheidungen unterliegen. Sind die Erwartungen über die Erträge der Ausbildungen gebildet, müssen die Erwartungen in Bildungsentscheidungen umgesetzt werden. In der ökonomischen Theorie wird dazu angenommen, dass das Individuum das Bildungsniveau wählt, das seinen Nutzen maximiert. Damit wird der Entscheidungsprozess eindeutig. Ohne diese Annahme kann - wie die Kritik von MANSKI oben zeigte - nicht eindeutig aus den Beobachtungen auf die zugrunde liegenden Entscheidungsmechanismen geschlossen werden. Im Vordergrund dieses Abschnittes steht deshalb die Frage, inwieweit davon auszugehen ist, dass die individuelle Entscheidung tatsächlich das Produkt einer freiwilligen, selbstbestimmten Auswahl darstellt, und ob das einzige Ziel die Maximierung des monetären Outputs ist, wie es in der ökonomischen Theorie heute angenommen wird. Um diese Fragen zu beantworten, sollen drei Sichtweisen über das Handeln des individuellen Agenten vorgestellt werden. ${ }^{99}$ Alle Ansätze sind extreme Auslegungen, die sich dennoch nicht gegenseitig ausschließen; sie sollen nur der Verdeutlichung unterschiedlicher Interpretationsmöglichkeiten dienen.

Der Strukturalistische Ansatz betrachtet die individuellen Verhaltensweisen als von externen Faktoren geleitet und lässt keinen Raum für individuelle Präferenzen, Erwartungen und Entscheidungsmechanismen. In mehr oder weniger

\footnotetext{
97 Vgl. Manski (1993), S. 47-56.

98 Vgl. die Schlussfolgerungen bei Das/Van Soest (2000), S. 31.

99 Für die folgenden Ausführungen über die verschiedenen theoretischen Ansätze vgl. Gambetta (1987), Kapitel 1, S. 7-29.
} 
extremen Auslegungen wird das menschliche Verhalten innerhalb gegebener Strukturen mechanisch abgeleitet. ${ }^{100}$

Ein Beispiel soll die Argumentation der Strukturalisten veranschaulichen. Angenommen, die Anzahl der Schüler, die einen Nebenjob ausführen, ist im Zeitverlauf gestiegen und dieser Anstieg soll erklärt werden. Aus strukturalistischer Sicht drängen sich z.B. die beiden folgenden Hypothesen auf: 1) Das Angebot an Studentenjobs am Arbeitsmarkt ist gestiegen. 2) Die Anzahl der Schüler aus armen Familien ist gestiegen. Der erste Erklärungsversuch macht die Ausblendung der individuellen Gründe deutlich. Eine Ausweitung des Jobangebots führt automatisch zu mehr arbeitenden Schülern. Das Vorhandensein von passenden Jobs ist zwar eine notwendige Erklärung für die Zunahme an arbeitenden Schülern, hinreichend ist sie aber nicht. Wird die Erklärung als ausreichend empfunden, steckt die implizite Annahme dahinter, dass die Schüler grundsätzlich nebenbei arbeiten wollen, der Arbeitsmarkt das aber bisher verhindert hat. Warum die Schüler arbeiten wollen, wird hier nicht hinterfragt. Der zweite Erklärungsansatz setzt zwar auf der Ebene des Individuums an: Wenn Kinder aus armen Familien in die Schule gehen, müssen sie nebenbei arbeiten. Die Armutshypothese wird als zwingender und einziger Grund für die gestiegene Anzahl an arbeitenden Schülern gesehen. Auch hier bleibt wieder der Entscheidungsprozeß auf individueller Ebene ausgeklammert, was die Sicht auf mögliche andere Erklärungsansätze verdeckt.

Der zweite Ansatz geht davon aus, dass die Individuen bestimmten Gründen folgen, die z. T. außerhalb ihres Bewusstseins liegen und deshalb nicht hinterfragt werden. GAMBETTA bezeichnet diesen Mechanismus der Entscheidungsfindung als Pushed-from-behind ${ }^{101}$. Die Gründe können unbewusst beeinflussen (konditioniertes Verhalten auf Stimuli) oder auch auf Traditionen beruhen (soziale Normen und Grenzen). Der individuelle Entscheidungsprozess wird auch hier kaum berücksichtigt. Das Individuum Akteur agiert aus Gründen, die außerhalb seiner persönlichen Wahrnehmung liegen oder als unantastbar gelten.

Die Anhänger dieses Pushed-from-behind Ansatzes würden die gestiegene Anzahl arbeitender Schüler ebenfalls mit dem gestiegenen Anteil von Arbeiterkindern in der Schule erklären. Den Grund dafür sähen sie aber darin, dass in dieser Bevölkerungsschicht der Arbeitserfahrung mehr Wert beigemessen wird als der ausschließlich schulischen Ausbildung. Ob die Anzahl der Arbeiterkinder tatsächlich im selben Zeitraum gestiegen ist, kann empirisch überprüft werden.

100 Einen extremen Ansatz vertritt Louis Althusser (1970), vgl. Gambetta (1987), S. 8.

101 Vgl. Gambetta (1987), S. 11. 
Aber selbst wenn die arbeitenden Kinder v. a. aus Arbeiterfamilien kommen, ist das Verhaltensmuster damit noch nicht belegt. Die strukturalistische These über die Einkommensbeschränkungen könnte ebenso stimmen.

Es ist sicher nicht auszuschließen, dass Individuen unter bestimmten Umständen wie Automaten funktionieren. Ein Set von behind-the-back Mechanismen kann bestimmt einen Beitrag zur Erklärung von Inter-Gruppen-Unterschieden leisten. Dennoch sollten nicht alle Entscheidungen unter Ausschluss ${ }^{102}$ der individuellen Präferenzen untersucht werden. Wenn Beobachter behaupten, die Individuen folgen ihnen unbekannten Gründen, behaupten sie implizit, ihre Wahrnehmungen seien denen der Handlungssubjekte überlegen. Diese Behauptung kann durch ein Informationsdefizit der Akteure durchaus zu rechtfertigen sein ${ }^{103}$, sollte aber nicht grundsätzlich angenommen werden.

Die beiden dargestellten Ansätze erachten das Individuum grundsätzlich als inaktiv. Es wird entweder in seiner Wahl durch den Mangel an relevanten Alternativen eingeschränkt, oder es wird durch Faktoren die sich seinem Einfluss und oft seiner Wahrnehmung entziehen in eine Richtung gedrängt. Der individuelle Entscheidungsprozess und seine Zielrichtung bleiben ausgeklammert.

Erst ein dritter Ansatz betrachtet das Individuum als fähig, alle möglichen Alternativen abzuwägen und unter Berücksichtigung der Zukunft eine individuelle Auswahl zu treffen. Er wird von CAMBETTA als Pulled-from-the-front View bezeichnet. Besser erscheint aber der Ausdruck Attracted-from-the-front, weil das Individuum sich seiner Präferenzen bewusst ist und seine Wahl aus den zur Verfügung stehenden Alternativen trifft. Diese Betrachtungsweise ist das übliche Vorgehen in der mikroökonomischen Theorie. Die Fähigkeit, bewusste Überlegungen bezüglich der eigenen Zukunft anzustellen, ist das Fundament der Rational Choice Theorie. ${ }^{104}$

Auch hier lassen sich verschiedene Ansichten über das menschlichen Verhalten unterscheiden. Innerhalb der ökonomischen Theorie hat sich eine extreme Auffassung herausgebildet, bei der das Individuum sein Verhalten bei gegebenen Präferenzen und Restriktionen der Marktsituation anpasst. Hier werden die individuellen Gründe zwar nicht vernachlässigt (wie in den beiden obigen Ansätzen), aber sie werden als gegeben, konstant und einer starren Regel folgend angenommen: Nutzenmaximierung. Jedes individuelle Verhalten kann aus den gegebenen Präferenzen und Nebenbedingungen hergeleitet werden. Diese Auf-

102 Es gibt gut Gründe dafür, individuelle Präferenzänderungen bei der Erklärung von Verhalten auszuschließen. Dazu sollten die als konstant angenommene Präferenzen aber explizit genannt werden. Vgl. dazu die folgenden Ausführungen zu der Rational Choice Theorie.

103 Vgl. die Ausführungen zu meritorischen Gütern bei Gottschalk (2001), S. 152-170.

104 Vgl. Gambetta (1987), S. 17. 
fassung von rationalem Verhalten ähnelt stark den beiden oben dargestellten Ansätzen.

HIRSCHMAN zeigte in seinem Buch Exit, Voice, and Loyalty, dass die Option Exit ${ }^{105}$ ineffizienter sein kann als die alternative Option, seine Stimme zu erheben (Voice), um die Situation zu ändern. ${ }^{106}$ Dennoch wird in der Rational Choice Theorie der Alternative Exit mehr Gewicht beigemessen. Das Individuum wird nur innerhalb seiner Präferenzstruktur als aktiv betrachtet. Seine Aktionen stellen weder das eigene Präferenzsystem noch die gegebenen Einschränkungen in Frage. Diese Vereinfachung in der ökonomischen Theorie ist notwendig, da die Präferenzbildung eines der schwierigsten Probleme in den Sozialwissenschaften darstellt. ${ }^{107}$

Es ist nicht zu erwarten, dass die Individuen die ihnen vorgegebenen Einschränkungen als naturgegeben betrachten. Dennoch werden sie einige Restriktionen als unveränderbar ansehen. Daher ist es sinnvoll, sie nur in gewissen Grenzen als rational handelnde Akteure zu betrachten. Auf empirischen Untersuchungen gestützt, argumentiert ELSTER, dass, obwohl nicht jedes menschliche Handeln rational oder vorsätzlich ist, „here is a well grounded presumption that this will typically be the case“108.

Um auch hier die Argumentation zu verdeutlichen, soll wieder das obige Beispiel herangezogen werden. Die Anhänger des rationalen Handlungsansatzes glauben, dass die Entscheidungen aufgrund irgendeiner rationalen Kalkulation getroffen wurden, in der persönliche Präferenzen mit der erwarteten zukünftigen Entwicklung berücksichtigt werden. Der Anstieg der Anzahl arbeitender Schüler kann demnach durch eine Senkung der Ertragsraten der Bildung ausgelöst werden. Die gesunkene Ertragsrate der Ausbildung ist immer noch hoch genug, um eine Investition in Bildung zu rechtfertigen. Aber sie ist auch genug gesunken, um die sofortigen Einkünfte aus der Nebentätigkeit attraktiver erscheinen zu lassen, als die gesamte Zeit dem Lernen zu widmen und auf zukünftige Einkommen zu warten.

Ein zweiter Erklärungsansatz könnte in einer allgemeinen Meinungsänderung (Präferenzen) der Schüler begründet liegen. Wenn diese der ökonomischen Unabhängigkeit von der Familie im Zeitverlauf mehr Wert beimessen, wird den sofortigen Einkommen durch den Nebenjob mehr Wert beigemessen als den

\footnotetext{
105 Auf das Beispiel Ausbildung bezogen heißt das z.B. das Verlassen oder Wechseln der Schule bei Unzufriedenheit.

106 Vgl. Hirschman (1970).

107 Vgl. Gambetta (1987), S. 20.

108 Elster (1979), S. IX.
} 
zukünftigen Einkommen aus der Ausbildung. Die optimale Zeitaufteilung verschiebt sich zugunsten des Nebenjobs.

Beide Erklärungsansätze sind theoretisch möglich und können gleichzeitig zu den Beobachtungen geführt haben. Ihre Relevanz muss empirisch überprüft werden. Da die Änderung der Präferenzen (mehr Unabhängigkeit) nur sehr viel schwieriger beobachtet werden kann, werden sie häufig als konstant angenommen. Ebenso schwierig beobachtbar sind Präferenzunterschiede zwischen Bevölkerungsgruppen.

Wenn die Präferenzstruktur aber als konstant und für alle gleich angenommen wird, ist sie zur Erklärung von Verhaltensänderungen und -unterschieden irrelevant. Erst wenn den Individuen unterschiedliche Präferenzen unterstellt werden, kann die Theorie für die Erklärung von unterschiedlichen Nachfrageverhalten herangezogen werden. Ist es aber im Extremfall nicht möglich, diese Präferenzen empirisch aufzudecken, können sie je nach Bedarf willkürlich zur Erklärung von Beobachtungen herangezogen werden. In der ökonomischen Theorie geht man von gegebenen Präferenzen aus, weil es oft nur so möglich ist, überprüfbare Hypothese aufzustellen.

Die Ausführungen zeigen, dass es a priori nicht möglich ist, zu entscheiden, welche der dargestellten Hypothesen plausibler als die anderen erscheint. Darüber hinaus ist nicht zu erwarten, dass die beobachtbaren individuellen Bildungsentscheidungen das Resultat von nur einem einzigen Entscheidungsmuster sind. Erstens ist es möglich, dass verschiedene Individuen ihre Entscheidung nach unterschiedlichen Mechanismen treffen. So kann z.B. eine Bevölkerungsgruppe rational abwägen, welche Bildungsentscheidung unter den gegebenen Umständen die höchste Ertragsrate hat, während eine andere Bevölkerungsgruppe so in soziale Normen verstrickt ist, dass sie die Vorteile der Ausbildung nicht richtig abschätzen kann. Zweitens kann ein Individuum gleichzeitig nach zwei Entscheidungsmechanismen handeln. Ein Kind aus einer Arbeiterfamilie könnte z.B. eine akademische Laufbahn ausschließen, weil dieser Bildungszweig ,nichts für die eigene Schicht' ist, aber im Bereich der verbleibenden Möglichkeiten durchaus nach rationalen Überlegungen auswählen. Drittens können an verschiedenen Ausbildungsstufen unterschiedliche Entscheidungsmechanismen herangezogen werden. Kinder der oberen Schichten können z.B. ,automatisch‘ auf Gymnasien geschickt werden, danach aber nach ihren Erfahrungen und Erwartungen individuell den weiteren Bildungsweg planen.

Es ist offensichtlich nicht realistisch anzunehmen, dass Individuen bei ihrer Bildungsentscheidung keine Wahl haben, wovon der Strukturalistische Ansatz ausgeht. Ebenso unrealistisch ist es, die individuellen Gründe derart abzuwerten, wie es die Anhänger des Pushed-from-behind-Ansatzes tun. Vielmehr bie- 
tet die in der Bildungsökonomie übliche Rational Choice Theorie den umfassendsten Ansatz, wenn die in den beiden anderen Ansätzen erwähnten Einflussfaktoren nicht ignoriert, sondern explizit als Restriktionen der individuellen Entscheidungsfindung berücksichtigt werden. Ebenso müssen die als gegeben angenommenen Präferenzen explizit genannt werden. Einer gegebenen Präferenzstruktur zu folgen, heißt nicht, sich der Entstehung und Wirkungen dieser Präferenzen nicht bewusst zu sein.

In den folgenden Untersuchungen soll daher zunächst davon ausgegangen werden, dass die Individuen versuchen, ihre persönlich angestrebten Ziele unter Berücksichtigung zukünftiger Entwicklungen zu erreichen. Gleichzeitig soll aber auch der mögliche Einfluss von pushing Faktoren, wie z.B. Normen, Traditionen und Werten, als Restriktionen berücksichtigt werden. Auch wenn nicht zu erwarten ist, dass diese Faktoren die Bildungsentscheidung allein bestimmen, können sie die Anzahl der möglichen Alternativen, unabhängig vom rationalen Entscheidungsprozeß, beeinflussen. Außerdem können diese unterbewussten Prozesse die Präferenzbildung beeinflussen. Schließlich sollen auch die strukturellen Beschränkungen, seien sie institutioneller Art (z.B. das Bildungssystem), persönlicher Art (z.B. die Einkommensverhältnisse) oder marktwirtschaftlicher Art (z.B. der Arbeitsmarkt) Berücksichtigung finden, weil sie die Alternativen der Individuen und auch der Gesellschaft als Ganzes einschränken können. ${ }^{109}$ 


\subsection{Auswirkungen auf die Modellanwendung}

Wie in Kapitel zwei und drei gezeigt wurde, gehen die ökonomischen Modelle davon aus, dass ein rationaler Investor solange in die Ausbildung investieren wird, bis die sinkenden Ertragsraten der Ausbildung den steigenden Kosten der Finanzierung entsprechen. Wie die obigen Ausführungen gezeigt haben, ist es nicht realistisch anzunehmen, dass jede individuelle Entscheidung aufgrund von rationalen Überlegungen bezüglich der Nutzen und Kosten und unter Bewusstsein aller relevanter Gründe und Motive getroffen wird. Ein Haushaltsvorstand wird kaum die exakte Ertragsrate der Bildungseinheit errechnen, bevor er seine Kinder in die Schule schickt.

Dennoch kann davon ausgegangen werden, dass vor jeder Bildungsentscheidung auch ein (u. U. auch unbewusstes) Nutzenkalkül steht. Daher bietet die in der Bildungsökonomie übliche Rational Choice Theorie den sinnvollsten Ansatz, die Bildungsnachfrage zu untersuchen.

Allerdings stellt die rationale Abwägung von Alternativen unter dem Ziel der Nutzenmaximierung sicher nicht den alleinigen Entscheidungsmechanismus dar. Wenn darüber hinaus noch von Heterogenität in der Erwartungsbildung auszugehen ist, kann von Beobachtungen nicht eindeutig auf den zugrunde liegenden Entscheidungsprozess in der Bildungsnachfrage geschlossen werden. Jede Interpretation hängt von den zur Vereinfachung getroffenen Annahmen ab. Die empirischen Ergebnisse sagen nichts über die Schlüssigkeit der Annahmen aus. Der einzige Weg aus diesem Dilemma scheint die explizite Untersuchung des Mechanismus zu sein, den Individuen zur Bildung ihrer Erwartungen über die Schulbildung nutzen. Dazu müssen Ökonomen auch subjektive Daten zur Einschulungsentscheidung erheben und in ihrer Analyse als Variablen berücksichtigen. ${ }^{110}$ Inwieweit dies möglich ist, und ob dieser Forderung bisher Folge geleistet wurde, soll in einem Überblick über die empirische Forschung zu den Bildungsdeterminanten in Entwicklungsländern im nächsten Kapitel geklärt werden.

110 Vgl. Manski (1993), S. 55-56. 


\section{Teil: Auswertung empirischer Studien zu den Deter- minanten von Bildungsinvestitionen}

\section{Schätzungen der Bildungsnachfrage?}

In der bildungsökonomischen Literatur gibt es eine Reihe von Untersuchungen zur individuellen Bildungsbeteiligung. Die meisten davon wurden für die USA durchgeführt und beziehen sich auf die höhere Bildung, die über die Schulpflicht hinaus geht. Interessanter für diese Arbeit sind die Schätzungen, die für Entwicklungsländer vorgenommen wurden. Obwohl sich die meisten der einschlägigen Untersuchungen auf ländliche Räume konzentrieren, sollen die Ergebnisse einiger Studien hier zusammengefasst werden, um ein Bild von der bisherigen empirischen Forschungsleistung auf diesem Gebiet zu zeichnen.

Zunächst ist anzumerken, dass es sich strenggenommen in den meisten Untersuchungen nicht um die Schätzung einer Bildungsnachfragefunktion handelt, obwohl in vielen Studien von Bildungsnachfrage gesprochen wird. Obwohl (wie in Kapitel 2 und 3 gezeigt wurde) theoretische Modelle zur Humankapitalbildung existieren, besteht eine sehr große Kluft zwischen diesen Modellen und ihren empirischen Tests. ${ }^{111}$ HANUSHEK kritisiert diese Art der empirischen Modellbildung folgenderweise: „....the analysis begins with a standard human capital investment model which compares expected benefits to the costs of schooling but then, in the empirical work, turns to a simple regression of college attendance on tuition." 112

Die meisten empirischen Arbeiten stehen nur in vager Relation zu den als Grundlage angegebenen theoretischen Ansätzen und integrieren eine Vielzahl von Variablen, für die es schwierig ist, eine strenge theoretische Herleitung zu präsentieren. Es besteht bei weitem kein Konsens darüber, welche Faktoren berücksichtigt und auf welche Art sie integriert werden sollen. Dennoch folgen die Untersuchungen einem ähnlichen Erklärungsmuster, das einen Vergleich der Ergebnisse erlaubt.

Fast alle Studien untersuchen die Determinanten der Schulbildung für Kinder im schulfähigen Alter und betrachten im theoretischen Modell die Familie als Entscheidungseinheit. Je nach Auswahl der abhängigen Variable werden unterschiedliche statistische Verfahren zur Berechnung der „Bildungsnachfrage“113

\footnotetext{
111 Vgl. Whitfield/Wilson (1991), S. 391.

112 Hanushek (1993), S.58.

113 Obwohl es sich in den meisten Fällen nicht um Nachfrageschätzungen handelt, wird der Begriff „Bildungsnachfrage“ in der folgenden Untersuchung benutzt, weil er sich in diesem Zusammenhang durchgesetzt hat und eine Umschreibung in vielen Fällen zu umfangreich würde.
} 
angewandt. Ein kurzer Überblick soll die am häufigsten angewandten Verfahren darstellen.

Wie schon erwähnt, handelt es sich bei den meisten Untersuchungen strenggenommen nicht um die Schätzung von Bildungsnachfragefunktionen. Dies liegt zu einem nicht unerheblichen Teil am Fehlen einer beobachtbaren Variablen, die eindeutig die Nachfrage nach Bildung zum Ausdruck bringt. Zunächst stellt sich das Problem, dass jeder zu beobachtende Bildungserfolg auch immer ein Ergebnis des Bildungsangebots darstellt. Man kann also nicht ohne weiteres auf die zugrunde liegende Nachfrage schließen. ${ }^{114}$

Sollen die Faktoren untersucht werden, die den Entscheidungsprozeß der Bildungsnachfrage der heutigen Schüler beeinflusst haben, stehen die Forscher vor einem weiteren Beobachtungsproblem. ${ }^{115}$ Als Ausdruck der Bildungsnachfrage könnte die Anzahl der abgeschlossenen Schuljahre als abhängige Variable verwendet werden. Allerdings sind die zu beobachtenden Werte nicht unbedingt als nachgefragte Mengen zu interpretieren. Für junge Menschen, die während der Befragung die Schule besuchten, sind die zu beobachtenden Werte höchstens die Untergrenze der Bildungsnachfrage, da nicht vorausgesehen werden kann, wie lange die Schüler die Schule weiterhin besuchen werden. Dieses Problem ist als Censored Observations bekannt.

Um Lernhindernisse zu identifizieren, werden auch die Bildungserfolge untersucht. Dabei wird je nach Datenlage auf Ergebnissen von Leistungstests, Abbrecherverhalten oder Klassenwiederholungen zurückgegriffen. Das Problem der Censored Observations stellt sich allerdings für alle Beobachtungen, die für eine noch in der Schule befindliche Zielgruppe erhoben werden. Eine Ausgrenzung dieser Gruppe aus der Stichprobe kommt nicht in Frage, da die Untersuchungsergebnisse die aktuelle Situation erklären sollen.

Außerdem kann die abhängige Variable oft nur diskrete Werte annehmen. Die Variable „Bildungspartizipation“ hat sogar binären Charakter, sie tritt als dichotome Variable auf. Es kann nur beobachtet werden, ob ein Kind die Schule besucht $(y=1)$ oder nicht $(y=0)$.

Die meisten Autoren entscheiden sich für die binäre Variable „Bildungspartizipation" und untersuchen den Zusammenhang zwischen der Teilnahme am Bildungssystem und einer Vielzahl von Variablen, die die Schüler selbst, deren Familien und das Bildungsangebot beschreiben.

114 Einige Autoren begegnen diesem Problem, indem sie Faktoren zum Bildungsangebot in ihre Schätzgleichungen integrieren, um diese Wirkung zu separieren.

115 Lillard/King zeigen die Probleme bei der Beobachtung der Bildungsnachfrage auf, diskutieren die üblichen Lösungswege und entwickeln eine eigene Methode zur Analyse von Bildungsentscheidungen in Haushalten. Vgl. Lillard/King (1984). 
Wegen der oben genannten Eigenarten der abhängigen Variablen kann keine einfache Regression nach der Methode der kleinsten Quadrate (OLS) geschätzt werden. Fast alle Autoren wenden daher als alternative Analyseform Qualitative Response Models (Kategoriale Regressionen) an. ${ }^{116}$ Dabei wird ein übliches Regressionsmodell angenommen.

Das Vorgehen wird hier kurz anhand der „Bildungspartizipation“ erläutert. Die abhängige Variable $\left(y^{*}\right)$ wird als latent bezeichnet. Was zu beobachten ist, ist eine Dummy-Variable. Die am häufigsten auftretenden Formen dieser Wahrscheinlichkeitsfunktionen in der bildungsökonomischen Literatur sind die Logitund die Probit-Analyse. Beide Formen unterscheiden sich durch die Annahme über den Fehlerterm $u$. Wenn die kumulative Verteilung von $u$ logistisch ist, wird von Logit-Modellen gesprochen. Wird die Verteilung als normal angenommen, handelt es sich um ein Probit-Modell. ${ }^{117} \mathrm{Da}$ sich die Verteilungsformen sehr ähneln, sind auch keine großen Unterschiede in den Ergebnissen beider Methoden zu erwarten. ${ }^{118}$

Ausgangspunkt ist jeweils eine übliche Regressionsfunktion, deren Berechnung aber nicht die tatsächlichen $y$-Werte ermitteln soll, sondern die Wahrscheinlichkeit, mit der die zu erklärende Variable den Wert 0 bzw. 1 annimmt $\left(P_{i}\right)$. Aus dieser Vorgabe lässt sich das Problem folgenderweise formulieren:

$$
P_{i}=F\left(\beta_{0}+\sum_{j=1}^{k} \beta_{j} x_{i j}\right),
$$

wobei $F$ die kumulative Verteilungsfunktion des Fehlerterms $u_{i}$ darstellt. Die funktionale Form von $F$ hängt also von den Annahmen über den Fehlerterm ab. Wird die Verteilung als logistic angenommen, erhalten wir das Logit-Modell, deren linke Seite als log-odds ratio bezeichnet wird.

Logit-Modell: $\quad \log \frac{P_{i}}{1-P_{i}}=\beta_{0}+\sum_{j=1}^{k} \beta_{j} x_{i j}$

Die so ermittelten Parameter $\beta_{i}$ geben also den Einfluss auf die log-odds ratio an. Um den Einfluss auf die Eintrittswahrscheinlichkeit der einen oder anderen Beobachtung $(y=0$ bzw. $y=1)$ zu ermitteln, muss diese Rate auf $P_{i}$ zurückgerechnet werden. ${ }^{119}$

Ein weiteres Analyseverfahren stellt das Tobit-Verfahren (Tobin's Probit) dar. ${ }^{120}$ Das einfache Modell kann angewendet werden, wenn die abhängige Variable

\footnotetext{
116 Zu den Analysemethoden vgl: Amemiya (1981), S. 1483-1536 und Maddala (1992).

117 Vgl. Maddala (1992), S. 327-329.

118 Vgl. Maddala (1992), S.328.

119 Ein anschauliches Beispiel bieten Demery/Ferroni/Grootaert (1993), Box 11.6, S. 192.

120 Vgl. Maddala (1992), S. 338-342.
} 
im Prinzip negative Werte annehmen könnte, diese aber nicht zu beobachten sind. Für alle $y>0$ wäre das übliche Regressionsverfahren anwendbar. Diese Phänomen nennt man truncated Beobachtungen. Negative Ausprägungen für $y$ sind im Zusammenhang mit der Bildungsnachfrage nicht möglich, weshalb das einfache Tobit-Modell hier nicht relevant ist. Allerdings ist das Modell erweiterbar. So kann eine Methode entwickelt werden, die berücksichtigt, dass die $y=0$ Beobachtungen das Ergebnis individueller Entscheidungen sind. So könnte die Entscheidung, die Kinder in die Schule zu schicken oder nicht, bei der Erklärung der Anzahl der absolvierten Schuljahre berücksichtigt werden. ${ }^{121}$

Die Ergebnisse der im folgenden zusammengefassten Untersuchungen sind natürlich nicht ohne weiteres vergleichbar. Den Schätzungen liegen unterschiedliche theoretische Ansätze zugrunde. Es wurden unterschiedliche abhängige Variablen ausgewählt. Die Variablen wurden unterschiedlich operationalisiert und mit verschiedenen statistischen Methoden überprüft. Dennoch lassen sich einige Gemeinsamkeiten aufzeigen.

Fast alle Autoren nennen als theoretischen Ansatz das oben dargestellte Modell der familiären Nutzenmaximierung von BECKER oder BEN-PORATH ${ }^{122}$. Die Modelle führen zu der Maximierung des Lebenseinkommens der Familie unter Berücksichtigung der Anzahl der Schuljahre der Kinder. Während die Details in diesen Optimierungsmodellen variieren können, bleibt die Grundidee immer der Trade-Off zwischen den entgangenen heutigen und den erwarteten zukünftigen Einkommen. ${ }^{123}$ Dennoch leitet kaum ein Autor die Schätzgleichung stringent aus dem theoretischen Modell ab. Bei den Schätzungen handelt es sich meistens um die Untersuchung der Wirkungen verschiedener Faktoren auf unterschiedlich definierte Indikatoren für den Bildungserfolg. Dabei werden die oben dargestellten ökonometrischen Analysemethoden angewendet.

Als erklärende Variable integrieren fast alle Autoren individuelle Charakteristika der Kinder, der Familie und einige Variablen zum Bildungsangebot in ihre Schätzgleichungen. Je nach Erklärungsansatz werden zusätzliche Variablen integriert, mehrere Verfahren angewendet und die Ergebnisse nach einzelnen Faktoren differenziert. Die Daten beziehen sich auf verschiedene Länder mit unterschiedlichen Bildungssystemen. Aber alle Autoren wählten die Kinder im schulfähigen Alter als Zielgruppe und werten Haushaltsdaten aus.

Unter Berücksichtigung dieser Hintergründe sollen im folgenden die Ergebnisse einiger Studien vorgestellt werden. Dabei soll aufgezeigt werden, inwieweit die

\footnotetext{
121 Vgl. dazu Psacharopoulos/Arriagada (1989), S. 691-695.

122 Vgl. die Ausführungen in Kapitel 3 und die dort genannten Literaturangaben.

123 Vgl. Lavy (1996), S. 293.
} 
aus der Theorie hergeleiteten Zusammenhänge untermauert werden können, und ob die Untersuchungen der grundsätzlichen Kritik der ökonomischen Verhaltenstheorie (rational choice) folge leisten. Außerdem werden Gemeinsamkeiten und Widersprüche sowie Untersuchungslücken aufgedeckt. 



\section{Kosten- und Nutzenfaktoren als Bildungsdeterminanten}

Wie die theoretischen Ausführungen in Kapitel 2 und 3 zeigen, entspricht beim optimalen Bildungsniveau die Ertragsrate den marginalen Finanzierungskosten. Da sich die Ertragsrate aus den Nutzen und den Kosten der Ausbildung zusammensetzt, müssten sich Unterschiede in diesen Größen bei sonst gleichen Bedingungen in unterschiedlichen Bildungsniveaus ausdrücken. Im folgenden soll daher untersucht werden, ob die Erkenntnisse empirischer Studien diesen Zusammenhang bestätigen.

\subsection{Kostenfaktoren als Bildungsdeterminanten}

Wie in den meisten Ländern wird auch in Peru das Bildungsangebot größtenteils von der öffentlichen Hand bereitgestellt. Um eine möglichst umfassende Bildungsteilnahme zu gewährleisten, ist die Basisausbildung (Primaria und Sekundaria) obligatorisch und wird nahezu kostenlos angeboten. Das heißt aber keineswegs, dass auf die Haushalte keine finanzielle Belastung durch den Schulbesuch der Kinder zukommt. Die Immatrikulationsgebühren und die Kosten für Schulbücher, Schreibmaterialien, Schuluniformen und Fahrtkosten müssen von den Schülern selbst oder deren Eltern getragen werden. Darüber hinaus muss der Haushalt die Opportunitätskosten des Schulbesuchs der Kinder tragen, da eine alternative Nutzung ihrer Zeit nicht mehr möglich ist.

\subsubsection{Direkte Kosten}

Im folgenden wird aus den empirischen Untersuchungen zusammengefasst, welcher Zusammenhang zwischen direkten Kosten und „Bildungsnachfrage“ zu erwarten ist. Dabei ist auffällig, dass nur wenige Autoren direkte Kostenfaktoren in ihre Schätzungen integrieren. Dies liegt sicher in der Datenlage begründet. Einige Autoren hatten Daten zu den Schulgebühren zur Verfügung, die meisten nutzen allerdings die Entfernung zur Schule als Indikator für eine höhere Kostenbelastung.

Die folgende Tabelle fasst zunächst die Ergebnisse von Untersuchungen zusammen, die die Schulgebühren als Variable in ihre Schätzungen integriert haben. Dabei ist zu beachten, dass als abhängige Variable (die die Bildungsnachfrage darstellen soll) meistens die Bildungsbeteiligung, einmal aber auch der Bildungserfolg gewählt wurde. 
Tabelle 6.1: Schulgebühren als Bildungsdeterminante

\begin{tabular}{|c|c|c|c|c|c|}
\hline Autor & Land & Zielgruppe & abhängige Variable & erklärende Variable & Ergebnis \\
\hline $\begin{array}{l}\text { Gertler/ } \\
\text { Glewwe, } 1992\end{array}$ & $\begin{array}{l}\text { Peru, länd- } \\
\text { lich }\end{array}$ & 10-18 Jahre & $\begin{array}{l}\text { Preiselastizitäten (Nachfrage } \\
=\text { Bildungsbeteiligung) }\end{array}$ & \multicolumn{2}{|c|}{$\begin{array}{l}\text { von }-0,18 \text { bis }-0,70, \text { steigt mit Ausgangsniveau, } \\
\text { Mädchen }>\text { Jungen, Arme }>\text { Reiche }\end{array}$} \\
\hline \multirow[t]{2}{*}{ Lavy, 1996} & \multirow{2}{*}{$\begin{array}{l}\text { Ghana, } \\
\text { Ländlich }\end{array}$} & \multirow[t]{2}{*}{ 5-12 Jahre } & jemals teilgenommen & Schulgebühren & nicht signifikant \\
\hline & & & Attainment (grades) & Schulgebühren & nicht signifikant \\
\hline \multirow{2}{*}{$\begin{array}{l}\text { Levison/Moe, } \\
1998\end{array}$} & \multirow{2}{*}{$\begin{array}{l}\text { Peru, } \\
\text { Ländlich }\end{array}$} & \multirow{2}{*}{$\begin{array}{l}\text { 10-19, } \\
\text { nur Mädchen }\end{array}$} & Bildungsbeteiligung, $\mathrm{j} / \mathrm{n}$ & Schulgebühren, & nicht signifikant \\
\hline & & & Bildungsbeteiligung, Stunden & Schulgebühren & nicht signifikant \\
\hline \multirow{2}{*}{$\begin{array}{l}\text { Skyt Nielsen, } \\
1998\end{array}$} & \multirow[t]{2}{*}{ Sambia } & \multirow{2}{*}{$\begin{array}{l}\text { 7-18, } \\
\text { Primarschule } \\
\end{array}$} & z. Z. Teilnahme, j/n & Schulgebühren & nicht signifikant \\
\hline & & & z. Z. Teilnahme, $\mathrm{j} / \mathrm{n}$ & Fehlen v. Gebühren & nicht signifikant \\
\hline \multirow[t]{2}{*}{ Weiler, 1989} & \multirow[t]{2}{*}{ USA } & \multirow{2}{*}{$\begin{array}{l}\text { Schüler nach der } \\
\text { High School }\end{array}$} & \multirow{2}{*}{$\begin{array}{l}\text { Wahl des Schultyps, } \\
\text { jeweils öffentlich, privat }\end{array}$} & Höhe der Gebühren & positiv \\
\hline & & & & Erhalt von Stipendien & positiv \\
\hline
\end{tabular}


Die Tabelle zeigt einheitlich die schwache Wirkung der direkten Kosten in Form von Schulgebühren. Kein Autor konnte für EL einen signifikanten Zusammenhang ermitteln.

Nur WEILER konnte für die USA einen positiven Zusammenhang zwischen der Wahl des Schultyps und der Höhe der Gebühren an der jeweiligen Schule feststellen. Für dieses unerwartete Ergebnis weist er allerdings auf eine mögliche Ursache in der Datenlage hin. ${ }^{124}$ Außerdem fand er die Schulauswahl auch direkt mit dem Erhalt von finanzieller Hilfe korreliert, was den Erwartungen eher entspricht und dem vorherigen Ergebnis widerspricht.

GERTLER/GLEWWE ermittelten unter anderem Preiselastiziäten für Sekundarbildung im ländlichen Peru und unterstützen den schwachen Zusammenhang zwischen Schulgebühren und Bildungsbeteiligung. Sie stellten fest, dass die Nachfrage (gemessen als Wahrscheinlichkeit des Schulbesuchs) generell relativ unelastisch auf die Anhebung von Schulgebühren reagiert. ${ }^{125}$ Allerdings steigt die Reagibilität mit steigendem Ausgangsniveau der Preise, und arme Haushalte reagieren stärker auf Preiserhöhungen als reiche. Die Elastizitäten für das unterste Einkommensquartil sind bei jedem Ausgangsniveau mehr als doppelt so hoch wie für das oberste Einkommensquartil. Die Anhebung relativ niedriger Schulgebühren lässt demnach noch keinen deutlichen Nachfragerückgang erwarten. Darüber hinaus sind die Elastizitäten für Mädchen in jedem Fall höher als für Jungen. Die Autoren ermittelten außerdem, dass die Anfahrtszeit einen signifikant negativen Einfluss auf die „Bildungsnachfrage“ in Peru hat, und wohlhabende Haushalte bereit sind, einen höheren finanziellen Beitrag (in Form von Gebühren) zur Reduzierung der Reisezeit zu leisten. Auch hier zeigt sich eine etwas höhere Zahlungsbereitschaft für Jungen.126 Die Untersuchung zeigt, dass für Qualitätsverbesserungen durchaus eine Zahlungsbereitschaft vorhanden ist. Auch Familien mit niedrigen Einkommen weisen noch eine positive Zahlungsbereitschaft auf, allerdings ist diese niedriger und sie reagieren auch vergleichsweise stärker auf Preisanhebungen.

Die folgende Tabelle fasst die Untersuchungen zusammen, die die Entfernung zur Schule als erklärende Variable in ihre Schätzung integriert haben.

124 Vgl. Weiler (1989), S. 280. Weiler führt einen Vergleich mehrerer statistischer Modelle für die Untersuchung von Bildungsentscheidungen an. In allen Varianten bleibt der unerwartete Effekt der Gebühren erhalten. Vgl. S. 281. Eine mögliche Erklärung für dieses Phänomen ist, dass der hoher Preis der Bildungseinrichtung deren Qualität signalisiert und damit die Nachfrage positiv beeinflusst.

125 Vgl. Gertler/Glewwe (1992), S. 185.

126 Vgl. Gertler/Glewwe (1992), S. 186. 
Tabelle 6.2: Entfernung als Bildungsdeterminante

\begin{tabular}{|c|c|c|c|c|c|}
\hline Autor & Land & Zielgruppe & abhängige Variable & erklärende Variable & Ergebnis \\
\hline Burney/Irfan, 1995 & Pakistan, ländlich & 10-25 Jahre & Bildungsbeteiligung, j/n & lokale Schule vorhanden & nicht signifikant \\
\hline \multirow{2}{*}{$\begin{array}{l}\text { Jamison/ } \\
\text { Lockheed } 1987\end{array}$} & \multirow[t]{2}{*}{ Nepal } & & Elterliche Bildungswunsch & lokale Schule vorhanden & nicht signifikant \\
\hline & & & $\begin{array}{l}\text { Bildungserfolg (Abweichung } \\
\text { vom } \varnothing \text { ) }\end{array}$ & lokale Schule vorhanden & nicht signifikant \\
\hline \multirow{2}{*}{$\begin{array}{l}\text { Levison/Moe, } \\
1998\end{array}$} & \multirow{2}{*}{$\begin{array}{l}\text { Peru, } \\
\text { Ländlich }\end{array}$} & \multirow{2}{*}{$\begin{array}{l}\text { 10-19, nur } \\
\text { Mädchen }\end{array}$} & Bildungsbeteiligung, j/n & lokale Schule vorhanden & positiv \\
\hline & & & Bildungsbeteiligung, j/n & Anfahrtszeit & nicht signifikant \\
\hline \multirow{3}{*}{$\begin{array}{l}\text { Skyt Nielsen, } \\
1998\end{array}$} & \multirow[t]{3}{*}{ Sambia } & \multirow{3}{*}{$\begin{array}{l}\text { 7-18, } \\
\text { Primarschule }\end{array}$} & z. Z. Teilnahme, j/n & lokale Schule vorhanden & nicht signifikant \\
\hline & & & z. Z. Teilnahme, j/n & Entfernung zur Schule & nicht signifikant \\
\hline & & & z. Z. Teilnahme, j/n & Zug. z. öff. Verkehrsm. & positiv \\
\hline $\begin{array}{l}\text { Gertler/ Glewwe, } \\
1992\end{array}$ & Peru, ländlich & 10-18 Jahre & $\begin{array}{l}\text { Bildungsteilnahme, örtliche } \\
\text { Schule }\end{array}$ & Anfahrtszeit & negativ \\
\hline $\begin{array}{l}\text { Glewwe/Grosh/ } \\
\text { Jacoby/Lockheed, } \\
\text { 1995127 }\end{array}$ & Jamaika & Primarschule & Auswahl der All-Age School & $\begin{array}{l}\text { Entfernung zur All-Age- } \\
\text { School relativ zur Primaria }\end{array}$ & negativ \\
\hline \multirow[t]{2}{*}{ Handa, 1996} & \multirow[t]{2}{*}{ Jamaika } & \multirow{2}{*}{$\begin{array}{l}\text { Sekundarschule } \\
\text { 13-19 Jahre }\end{array}$} & Teilnahme, j/n & Entfernung zum Bus & negativ \\
\hline & & & akademischer Schultyp, j/n & Entfernung zum Bus & nicht signifikant \\
\hline \multirow[t]{2}{*}{ Lavy, 1996} & \multirow[t]{2}{*}{ Ghana, ländlich } & \multirow[t]{2}{*}{ 5-12 Jahre } & jemals teilgenommen & \multirow{2}{*}{$\begin{array}{l}\text { Entfernung zur Schule, } \\
\text { auch höhere Level }\end{array}$} & negativ \\
\hline & & & Attainment (grades) & & negativ \\
\hline Weiler, 1989 & USA & $\begin{array}{l}\text { Schüler nach der } \\
\text { High School }\end{array}$ & $\begin{array}{l}\text { Wahl des Schultyps, jeweils } \\
\text { öffentlich, privat }\end{array}$ & Entfernung & negativ \\
\hline
\end{tabular}

127 Vgl. Glewwe/Grosh/Jacoby/Lockheed (1995), S. 244. 
Zum Zusammenhang zwischen der Entfernung zur Schule und der „Bildungsnachfrage kann vermutet werden, dass eine weitere Entfernung zu höheren Kosten des Schulbesuchs führt und damit die „Bildungsnachfrage“ senkt. Allerdings können die Entfernungsdeterminanten nicht eindeutig als Hilfsindikatoren für höhere direkte Fahrtkosten betrachtet werden. Ein Zusammenhang der Variablen kann ebenso auf Opportunitätskosten durch den höheren Zeitbedarf zurückzuführen sein. Die in der Tabelle zusammengetragenen Ergebnisse bedürfen daher einer sorgfältigen Interpretation.

Die Tabelle zeigt ein relativ einheitliches Bild. Die meisten Autoren konnten dem Vorhandensein einer lokalen Bildungseinrichtung keinen Einfluss auf die Bildungsentscheidung nachweisen, während sich die Entfernung zur Schule in den meisten Studien negativ auf die Bildungsentscheidung auswirkte. Dieses Ergebnis überrascht zunächst, da doch das Vorhandensein einer Schule den Anfahrtsweg automatisch verkürzt. Die Widersprüche treten dann besonders deutlich hervor, wenn mit dem gleichen Datensatz beide Variablen auf ihren Einfluss überprüft wurden.

LEVISON/MOE konnten als einzige Autoren einen deutlichen positiven und signifikanten Einfluss der Existenz einer Schule in der Nachbarschaft auf den Schulbesuch nachweisen. Allerdings war der Anfahrtszeit kein signifikanter Einfluss nachzuweisen. Da die Autoren außerdem keinen Einfluss der Schulgebühren auf die Bildungsbeteiligung nachweisen konnten ${ }^{128}$, liegt hier die Vermutung nahe, dass der Zusammenhang zwischen der Existenz der Schule und dem Schulbesuch eher auf die Opportunitätskosten zurückzuführen ist.

Auch LAVY ermittelte für Kinder im grundschulfähigen Alter im ländlichen Ghana, dass die Schulgebühren keinen nachweislichen Einfluss auf die Bildungsbeteiligung und den Bildungserfolg haben. Wegen der relativ niedrigen Schulkosten öffentlicher Primarschulen betrachtet LAVY allerdings auch die Entfernung zur nächsten Bildungseinrichtung und damit die Reisekosten als wichtigsten Zugangsfaktor. ${ }^{129}$ Hier ermittelte er einen signifikant negativen Einfluss auf die "Bildungsnachfrage" nach Primarbildung. Auffällig ist bei seiner Untersuchung, dass vor allem auch der Entfernung zu Post-Primary Bildungseinrichtungen eine entscheidende Rolle bei der Einschulungsentscheidung und bei den Bildungserfolgen in der Primaria spielen. Dieses Ergebnis unterstützt LAVYs Vermutung, dass die Primaria nur als notwendige Voraussetzung für weitere Bil-

128 Vgl. Levison/Moe (1998), S.350 und 353. In einer Probit-Analyse mit Berücksichtigung der Familienstruktur ist der Effekt der Kosten negativ. Unter Ausschluss der Familienvariablen wird der Effekt stärker und positiv. In beiden Fällen sind die Ergebnisse nicht signifikant.

129 Vgl. Lavy (1996), S. 296. Da die direkten Gebühren in Ghana sehr niedrig sind, spielen die sonstigen direkten Kosten eine größere Rolle. Deshalb konzentriert Lavy seine Untersuchungen auf die Entfernung. 
dung betrachtet wird. Eine Investition ist daher nur lohnend, wenn die Kinder später auch eine höhere Bildungseinrichtung erreichen können.

Diese These wird auch durch die Erkenntnis von SKYT NIELSEN unterstützt, die zwar für keinen der Kostenfaktoren einen Effekt auf die Ausbildung ermitteln konnte, aber für den Zugang zu öffentlichen Verkehrsmitteln einen signifikanten positiven Koeffizienten erhielt. ${ }^{130}$ Auch hier scheint die Offenheit und die Möglichkeit, Chancen außerhalb der Gemeinde (z.B. höhere Bildung) wahrnehmen zu können, stärker zu wiegen.

Wenn die Basisausbildung nur als notwendige Voraussetzung für höhere Bildung betrachtet wird, lässt sich auch die unterschiedliche Wirkungsweise der beiden Variablen ,lokale Schule‘ und ,Entfernung zur Schule‘ erklären. Das Vorhandensein einer lokalen Basisschule hat für sich keinen großen Wert für die Bildungsentscheidung, wenn später keine höhere Bildungseinrichtung für die Schüler erreichbar ist. Wenn allerdings die Entfernungsvariable nicht nur die Entfernung zu einer Primarschule misst, sondern zur nächsten Stadt mit umfassenden Bildungsangebot, erscheint der Einfluss der Variable erklärbar.

Auch BURNEY/IRFAN ${ }^{131}$ und JAMISON/LOCKHEED 132 fanden heraus, dass das Vorhandensein einer lokalen Schule keinen signifikanten Einfluss auf die Bildungsentscheidung hat. Daraus kann gefolgert werden, dass der Aufbau von Primarschulen allein das Problem der geringen Bildungsbeteiligung in Entwicklungsländern nicht lösen kann. ${ }^{133}$

Ob sich der negative Effekt der Entfernung zur Schule nur aus den direkten Fahrtkosten (also Fahrkarten) ergibt, oder ob vor allem die Fahrtzeit Opportunitätskosten generiert, geht aus den Berechnungen meistens nicht hervor. Hier gibt allerdings HANDA einen deutlichen Hinweis. Er nahm für jamaikanische Jugendliche die Entfernung zum Bus als erklärende Variable und ermittelte einen signifikanten negativen Einfluss auf die Wahrscheinlichkeit ihrer Teilnahme an der Sekundarbildung. ${ }^{134} \mathrm{Da}$ nicht zu erwarten ist, dass neben der benötigten Zeit für den Weg zum Bus noch Out-of-Pocket-Ausgaben entstehen, handelt es sich hierbei eher um Opportunitätskosten.

Wenn die obigen Ergebnisse betrachtet werden, fällt zunächst auf, dass den Schulgebühren in keiner der Untersuchungen ein signifikant negativer Einfluss auf die Bildungsbeteiligung nachgewiesen werden konnte. Das kann damit zu-

\footnotetext{
130 Vgl. Skyt Nielsen (1998), S. 16-20.

131 Vgl. Burney/Irfan (1995), S. 39 und 40.

132 Vgl. Jamison/Lockheed (1987), S. 294-302.

133 Vgl. Burney/Irfan (1995), S. 34.

134 Vgl. Handa (1996), S. 567.
} 
sammenhängen, dass in den meisten Entwicklungsländern für die Basisausbildung kaum oder nur sehr geringe Gebühren erhoben werden. Die direkten Kosten haben damit einen geringen Stellenwert in der Investitionsentscheidung von Familien.

Die meisten Untersuchungen habe den Einfluss von sonstigen Kosten wie z.B. für Schulbücher, Uniformen und so weiter nicht untersucht, was zum Teil in der Datenlage begründet liegt. Während die meisten Untersuchungen für das Vorhandensein lokaler Bildungseinrichtungen keinen Einfluss auf die Bildungsentscheidung nachweisen konnten, konnten einige zumindest der Entfernung zur nächsten Schule den erwarteten negativen Effekt nachweisen. Ein langer Schulweg scheint sich tendenziell negativ auf die „Bildungsnachfrage“ auszuwirken. Geht man davon aus, dass ein längerer Anfahrtsweg auch höhere Transportkosten verursacht, können die Ergebnisse als Hinweise auf die Wirksamkeit von direkten Kosten betrachtet werden. Allerdings ist dies nur ein Teil der Erklärung. Die Reisekosten beinhalten neben den Fahrpreisen auch den Zeitbedarf für längere Anfahrtswege. Da den direkten Kosten in Form von Schulgebühren kaum Einfluss auf die „Bildungsnachfrage“ nachgewiesen werden konnte, liegt auch bei den Schulwegen die Vermutung nahe, dass die Opportunitätskosten durch den höheren Zeitbedarf die größere Rolle spielen.

Die Ergebnisse geben außerdem Hinweise darauf, dass die Kostenabwägung nicht allein für die Primarbildung durchgeführt wird. Wichtig scheint ebenfalls zu sein, ob auch eine weiterführende Schule zu vernünftigen Kosten zu erreichen ist.

\subsubsection{Opportunitätskosten}

Wie schon erwähnt, entstehen den Haushalten über die direkten Kosten hinaus auch Opportunitätskosten durch den Schulbesuch der Kinder. ${ }^{135}$ Wie die Untersuchungen zu den direkten Kosten zeigten, liegt die Vermutung nahe, dass die Opportunitätskosten den wichtigeren Kostenfaktor darstellen. Die Opportunitätskosten entstehen, weil die in der Schule verbrachte Zeit einer alternativen Verwendung nicht mehr zur Verfügung steht. Je nach potentieller Verwendung können die Auswirkungen sehr unterschiedlicher Art sein. Bei individuellem Kalkül sind die Opportunitätskosten des Schulbesuchs die Einkommen, die das Kind erzielen könnte, wenn es statt zur Schule arbeiten ginge. Beim Kalkül der Familie als Entscheidungseinheit können dies auch Kosten sein, die anderen Familienmitgliedern entstehen. Hierbei kann es sich z.B. um ausfallende Ver- 
dienste der Mutter (Tante, Oma) handeln, wenn sie sich wieder selber um den Haushalt oder die kleineren Kinder kümmern muss, während die älteren Geschwister in der Schule sind. ${ }^{136}$ Auch die Anstellung von Hilfskräften zur Kompensation der fehlenden Unterstützung durch die Schulkinder verursacht Kosten, die der Ausbildung zuzurechnen sind.

Da die Opportunitätskosten nicht direkt messbar sind, werden in der Literatur ganz unterschiedliche Beobachtungen herangezogen, um einen Hinweis auf Wirksamkeit dieser Kosten bei der Bildungsentscheidung zu erhalten.

\subsubsection{Entgangene Einkommen}

Nach RosENZWEIG stellen die Opportunitätskosten den größten Kostenfaktor des Schulbesuchs in Entwicklungsländern dar. Er argumentiert, dass in Ländern niedriger Einkommen eine bezahlte Tätigkeit die beste Alternative zum Schulbesuch ist. 137

GeRTLeR/GlewWe haben für das ländliche Peru zunächst einige Daten zur durchschnittlichen Arbeitsbelastung der Kinder und Jugendlichen gesammelt. ${ }^{138}$ Von den Nicht-Schülern arbeiten die 10-14-jährigen Jungen durchschnittlich zwischen 21 und 25 Stunden die Woche außerhalb des eigenen Haushaltes. Schüler dieser Altersgruppe arbeiten dagegen nur ca. 8 Stunden wöchentlich am Arbeitsmarkt. Die 15-18-jährigen Nicht-Schüler arbeiten sogar 27 bis 36 Stunden wöchentlich. Bei Schülern reduziert sich die Arbeitszeit auf 13 Stunden. Während die Mädchen insgesamt sogar mehr Zeit für nicht-schulbezogene Tätigkeiten aufwenden, leisten sie einen größeren Teil ihrer Arbeitszeit im eigenen Haushalt. Aber auch ihre Arbeitszeit außerhalb des eigenen Haushaltes liegt bei Nicht-Schülerinnen zwischen 12 und 28 Stunden wöchentlich und bei Schülerinnen nur zwischen 5 und 12 Stunden. Im ländlichen Peru scheint das Ausüben einer bezahlten Tätigkeit durchaus eine Alternative zum Schulbesuch darzustellen. Der Schulbesuch scheint die Arbeitszeiten und damit die Verdienstmöglichkeiten deutlich zu beeinflussen.

PSACHAROPOULOS/ARRIAGADA zeigen diesen Zusammenhang auch für Brasilien. Sie ermittelten, dass Kinder die arbeiten müssen, mit signifikant niedrigerer Wahrscheinlichkeit eingeschult werden als ihre Altersgefährten. Sie weisen au-

136 Denkbar wäre auch der umgekehrte Fall: Eine Mutter (oder ältere Schwester) kann sich wieder ihrem Job widmen, wenn ein kleineres Kind während des Schulbesuchs nicht mehr beaufsichtigt werden muss. Das so durch den Schulbesuch realisierbare Einkommen wird auf der Nutzenseite als positiver externer Effekt des Schulbesuchs verbucht.

137 Vgl. Rosenzweig (1990), S. 41. Rosenzweig ermittelte die Kinder-Lohn-Elastizität für die Philippinen, Indonesien und Indien. Vgl. die Tabelle auf S. 48.

138 Vgl. zu den folgenden Zahlen die Tabelle 4, S. 175 in: Gertler/Glewwe (1992). 
ßerdem eine fünffach höhere Wahrscheinlichkeit auf, die Schule abzubrechen. ${ }^{139}$

Wie die Ausführungen in Kapitel 2 gezeigt haben, müssten für die Opportunitätskosten einer zusätzlichen Bildungsstufe die Einkommen angesetzt werden, die ein Absolvent der nächsten niedrigeren Bildungsstufe am Arbeitsmarkt erhalten würde. Da die Haushalte aber keine Alters-Einkommensprofile zur Verfügung haben, sind ihre Vermutungen über die potentiellen Einkommen der Kinder relevant. Diese sind in Haushaltsdaten aber kaum enthalten. Deshalb werden zur Untersuchung des Einflusses der Opportunitätskosten auf die Bildungsentscheidung unterschiedliche Hilfsindikatoren herangezogen. Der Anteil der arbeitenden Haushaltsmitglieder (ausschließlich des beobachteten Individuums) kann als ein solcher Indikator betrachtet werden. Er stellt ein Maß für die Aussichten am Arbeitsmarkt dar. Nur wer eine reale Chance hat, auf dem Arbeitsmarkt eine bezahlte Tätigkeit zu bekommen, kann das potentielle Einkommen als Alternative zum Schulbesuch betrachten. Je unwahrscheinlicher seine Verdienstaussichten sind, desto niedriger sind die Opportunitätskosten anzusetzen. Zum anderen kann eine hohe Beteiligungsrate aber auch den Bedarf der Familie an zusätzlichem Einkommen darstellen. ${ }^{140}$ Wenn z.B. viele Niedrigverdiener das Existenzminimum der Familie nicht erbringen können, fällt der Verzicht auf das potentielle Einkommen eines Schülers sehr schwer. In beiden Fällen stellt die Variable einen Indikator für hohe Opportunitätskosten dar.

Nur wenige Autoren haben diesen oder ähnliche Indikatoren in ihre Untersuchungen integriert. Die folgende Tabelle fasst die Ergebnisse zusammen.

Tabelle 6.3: Entgangene Einkommen als Bildungsdeterminante

\begin{tabular}{l|l|l|l|l|l} 
Autor & Land & Zielgr. & abh. Variable & erklärende Variable & Ergeb. \\
\hline $\begin{array}{l}\text { Burney/ Irfan, } \\
1995\end{array}$ & $\begin{array}{l}\text { Pakistan } \\
\text { ländlich }\end{array}$ & 10-25 J. & $\begin{array}{l}\text { Bildungsbeteiligung, } \\
\text { j/n }\end{array}$ & $\begin{array}{l}\text { Arbeitsmarktbeteili- } \\
\text { gung der Familie }\end{array}$ & $\begin{array}{l}\text { negativ } \\
\mathrm{m}>\text { w }\end{array}$ \\
\hline $\begin{array}{l}\text { Skyt Nielsen, } \\
1998\end{array}$ & Sambia & $\begin{array}{l}7-18, \\
\text { Primaria }\end{array}$ & Z. Z. Teilnahme, j/n & Arbeitsmarktbet. & negativ \\
\cline { 4 - 6 } & Z. Teilnahme, j/n & $\begin{array}{l}\text { Arbeitslosigkeit des } \\
\text { Haushaltsvorstands }\end{array}$ & negativ \\
\hline Lavy, 1996 & $\begin{array}{l}\text { Ghana, } \\
\text { ländlich }\end{array}$ & $5-12 \mathrm{~J}$. & jemals teilgen., j/n & $\begin{array}{l}\varnothing \text { regionale Lohnrate } \\
\text { erwachsener Männer }\end{array}$ & positiv \\
\cline { 6 - 6 } & & Attainment (grades) & positiv \\
\hline
\end{tabular}

Nur BURNEY/IRFAN und SKYT NIELSEN integrierten die Arbeitsmarktbeteiligung der Familie in ihre Schätzgleichungen. ${ }^{141}$ Beide Untersuchungen belegen einen negativen Effekt dieser Variable auf die Bildungsbeteiligung. Wenn also eine große Anzahl Familienmitglieder einer bezahlten Tätigkeit nachgeht, sinkt die Wahrscheinlichkeit, dass das Kind die Schule besucht.

\footnotetext{
139 Vgl. Psacharopoulos/Arriagada (1989), S. 691 und 699.

140 Vgl. Burney/Irfan (1995), S. 32.

141 Dabei wird die Arbeitsmarktbeteiligung der Familie exklusive des betrachteten Kindes ermittelt.
} 
Bei BURNEY/IRFAN ist der Einfluss auf die Jungen wesentlich höher als auf die Mädchen. Für Nicht-Farm Haushalte wird der Unterschied zwischen den Geschlechtern kleiner, weil die Mädchen einen deutlich höheren Koeffizienten aufweisen als ihre Geschlechtsgenossinnen in Farmhaushalten. ${ }^{142}$ Dieses Ergebnis lässt vermuten, dass auch in Pakistan (wie in Peru, siehe oben) die Jungen eher einer bezahlten Tätigkeit nachgehen, während die Mädchen eher im Haushalt helfen. Solange innerhalb der eigenen Familie viel Arbeit anfällt, werden die Mädchen zur Unterstützung im Haushalt gebraucht. Ihre Opportunitätskosten in Form von entgangenen Einkommen sind daher relativ gering. Ihr Wert auf dem Arbeitsmarkt steigt aber, wenn die Arbeitsintensität in den Haushalten sinkt.

SKYT NiELSEN fand heraus, dass auch die Arbeitslosigkeit des Haushaltsvorstandes einen negativen Einfluss auf die Bildungsbeteiligung der Kinder hat. ${ }^{143}$ Dies scheint der obigen Aussage zunächst zu widersprechen, da damit die schwierige Situation auf dem Arbeitsmarkt dokumentiert wird. Die Kinder scheinen hier den Verdienstausfall kompensieren zu müssen, was ebenfalls ihre Opportunitätskosten erhöht. Sowohl verlockende Aussichten auf dem Arbeitsmarkt als auch zusätzlicher Bedarf an Einkommen generieren Opportunitätskosten durch den Schulbesuch.

LAVY nutzte in seiner Untersuchung über die Ausbildung von Grundschülern im ruralen Ghana die durchschnittliche regionale Lohnrate erwachsener Männer (vor allem in der Landwirtschaft) als Maß für die Opportunitätskosten des Schulbesuchs. ${ }^{144}$ Er ermittelt allerdings eine positive Korrelation zwischen den Löhnen und dem Schulbesuch der Kinder. In Ghana ist der Lernerfolg der Primaria so gering, dass diese Bildungsstufe in erster Linie als Zugangsvoraussetzung für weitere Ausbildung betrachtet wird. Der urbane Arbeitsmarkt betrachtet die Primarausbildung deshalb auch als keine Ausbildung und bietet kaum Verdienstmöglichkeiten für Menschen mit Primarschul- oder weniger Ausbildung. ${ }^{145}$ Wenn man diese Erkenntnisse auf das ländliche Ghana übertragen kann, ist die durchschnittliche Lohnrate einer Region eher ein Maß für die zukünftigen Verdienstaussichten in der Region und damit ein Maß für die Erträge der Ausbildung, was den positiven Zusammenhang erklären könnte. ${ }^{146}$

142 Vgl. Burney/Irfan (1995), S. 32-33.

143 Vgl. Skyt Nielsen (1998), Tabelle 5, S. 17.

144 Vgl. Lavy (1996), S. 300 und 301.

145 Vgl. Lavy (1996), S. 296. Diese Aussage folgt aus von Glewwe ermittelten Ertragsraten, die für die Primaria in Ghana nahezu Null sind. Vgl. Glewwe (1996), S. 267-290.

146 In der Herleitung der Nachfragefunktion sollten die Erwachsenenlöhne ursprünglich auch als Ertragsmaß neben den Kinderlöhnen als Maß für die Opportunitätskosten eingehen. Vgl. Lavy (1996), S. 297. Wegen der Datenlage musste auf die Variable Kinderlohn verzichtet werden. Die Erwachsenen- 
Wird die Qualität der Mittelschulen in der Gleichung berücksichtigt, sinkt der Einfluss der Löhne und verliert seine Signifikanz. Dies kann einerseits ein Hinweis darauf sein, dass die Löhne als Ertragsaussichten betrachtet werden. Wenn die Primaria als Zugangsvoraussetzung für die Mittelschule betrachtet wird, spielen die Ertragsaussichten erst mittelbar nach deren Qualität eine Rolle. Andererseits könnte dies aber auch den Kostencharakter der Löhne bestätigen. Für bessere zu erwartende Qualität werden auch höhere Kosten in Kauf genommen, bevor die Bildungsbeteiligung sinkt.

Die Untersuchungen zeigen, wie schwierig es ist, aus Beobachtungen auf die Rolle der entgangenen Einkommen bei der Bildungsentscheidung zu schließen. Nur wenige Autoren integrieren eine Ersatzvariable, die auf die Opportunitätskosten in Form von entgangenen Einkommen hinweisen könnte. Dennoch lassen die Ergebnisse den Schluss zu, dass der Verzicht auf eigene Einkommen der Schüler deren „Bildungsnachfrage“ negativ beeinflusst. Diese Opportunitätskosten beeinflussen die Bildung der Jungen stärker als die der Mädchen. Erklärbar ist dies durch die oben genannte Aufteilung der Arbeitskraft zwischen Jungen und Mädchen. Während Jungen häufiger einer bezahlten Tätigkeit nachgehen, helfen die Mädchen häufiger im Haushalt. Ob sich dies in den Untersuchungen zu den häuslichen Opportunitätskosten bestätigen lässt, wird im folgenden Abschnitt untersucht.

\subsubsection{Fehlende Mithilfe im Haushalt}

Hier sollen die empirischen Ergebnisse bezüglich der Opportunitätskosten durch die fehlende Mithilfe der Kinder in den Haushalten oder Familienbetrieben zusammengetragen werden. Rein theoretisch müssten dafür die Kosten herangezogen werden, die entstehen, wenn der Arbeitsausfall der Kinder durch bezahlte Kräfte ausgeglichen würde. ${ }^{147} \mathrm{Da}$ die Kosten häufig aber im Verzicht liegen oder die Einstellung von Hilfskräften von den Familien nicht direkt mit der Ausbildung in Zusammenhang gebracht werden, lassen sich diese Kosten kaum erfragen. Die in der folgenden Tabelle dargestellten Ergebnisse beziehen sich daher auf Hilfsindikatoren.

löhne wurden als Annäherung herangezogen, weil sich - dort wo beide Angaben enthalten waren eine hohe Korrelation zwischen beiden Größen feststellen ließ.

147 Vgl. die Ausführungen zu den Kosten der Ausbildung in Kapitel 2.1.2. 
Tabelle 6.4: Tätigkeit des Haushaltsvorstandes als Bildungsdeterminante

\begin{tabular}{|c|c|c|c|c|c|}
\hline Autor & Land & Zielgruppe & abhängige Variable & erklärende Variable & Ergebnis \\
\hline \multirow{3}{*}{$\begin{array}{l}\text { Psacharop./ } \\
\text { Arriagada, } 1989\end{array}$} & \multirow[t]{3}{*}{ Brasilien } & \multirow{3}{*}{ 7-14 Jahre } & Teilnahme, j/n. & Vater ist Farmer & negativ \\
\hline & & & Erreichungsgrad & Vater ist Farmer & negativ \\
\hline & & & Teilnahme, j/n & Vater ist Angestellter & positiv \\
\hline \multirow[t]{2}{*}{ De Vreyer, 1993} & \multirow[t]{2}{*}{$\begin{array}{l}\text { Elfenbein- } \\
\text { küste }\end{array}$} & \multirow[t]{2}{*}{ 5-25 Jahre } & Anzahl Schuljahre & $\begin{array}{l}\text { HH-Vorstand ist abhängig be- } \\
\text { schäftigt }\end{array}$ & positiv \\
\hline & & & Anzahl Schuljahre & HH-Vorstand ist selbständig & negativ \\
\hline Skyt Nielsen, 1998 & Sambia & $7-18$ & z. Z. Schulbesuch & $\mathrm{HH}$ in Landwirtschaft tätig & positiv \\
\hline \multirow[t]{2}{*}{ Burney/Irfan, 1995} & \multirow{2}{*}{$\begin{array}{l}\text { Pakistan, } \\
\text { ländlich }\end{array}$} & \multirow{2}{*}{$\begin{array}{l}\text { 10-25 Jahre } \\
\text { nur Farmer }\end{array}$} & Bildungsbeteiligung, j/n & Landgröße (acres) & nicht signifikant \\
\hline & & & Bildungsbeteiligung, j/n & Vater ist Landeigner & positiv \\
\hline \multirow[t]{3}{*}{ Jacoby, 1994} & \multirow[t]{3}{*}{ Peru } & \multirow[t]{3}{*}{ 7-12 Jahre } & Wiederholungswahrscheinlichkeit & Betriebsvermögen & positiv \\
\hline & & & Wiederholungswahrscheinlichkeit & Einkommen aus selbst. Arbeit & nicht signifikant \\
\hline & & & Wiederholungswahrscheinlichkeit & sonstiges Eink. & negativ \\
\hline \multirow[t]{3}{*}{ Levison/Moe, 1998} & \multirow{3}{*}{$\begin{array}{l}\text { Peru, } \\
\text { ländlich }\end{array}$} & \multirow{3}{*}{$\begin{array}{l}\text { 10-19, nur } \\
\text { Mädchen }\end{array}$} & wöchentliche Arbeitszeit im HH & Landbesitz & steigt um 3,2 h \\
\hline & & & Bildungsbeteiligung, j/n & Landbesitz & nicht signifikant \\
\hline & & & wöchentliche Zeit in der Schule & Landbesitz & nicht signifikant \\
\hline
\end{tabular}


In den vorliegenden empirischen Untersuchungen wurden verschiedene Variablen getestet, um den Einfluss der Opportunitätskosten messen zu können. Zunächst kann die Tätigkeit des Haushaltsvorstandes als guter Indikator für die Opportunitätskosten aufgefasst werden, wenn man davon ausgeht, dass sie den Arbeitsanfall im Haushalt stark beeinflusst. Die These dazu ist, dass Haushalte mit Familienbetrieben stärker auf die Mithilfe ihrer Kinder angewiesen sind.

PSACHAROPOULOS/ARRIAGADA ${ }^{148}$ und DE VREYER ${ }^{149}$ ermittelten tatsächlich, dass die Ausbildung von Kindern von Landwirten und selbständigen Vätern negativ beeinflusst wird, während die Ausbildung von Kindern von Angestellten positiv beeinflusst wird.

Auch BURNEY/IRFAN ermitteln deutlich niedrigere Einschulungsraten für FarmHaushalte im ländlichen Pakistan. Während 35\% der 10-16jährigen Kinder von Nicht-Farmern in die Schule gehen, sind dies nur 23\% der Farmerkinder. Die Mädchen liegen in beiden Fällen weit unter diesem Schnitt. ${ }^{150}$ Für die FarmHaushalte schätzten die Autoren außerdem den Einfluss der Landgröße und der Besitzverhältnisse auf die Einschulungsentscheidung151. Für die Landgröße konnten sie keinen signifikanten Einfluss ermitteln. Wenn das Land allerdings im Besitz der eigenen Familie ist, ist der Einfluss auf die Bildungsbeteiligung der Kinder positiv. Diese Ergebnisse scheinen zunächst im Widerspruch zu den obigen Ergebnissen zu stehen. Allerdings handelt es sich hier nur um die Stichprobe der Farmer. Für diese Gruppe steht der Landbesitz und die Landgröße vor allem für den sozioökonomischen Status der Familie und weniger für die Arbeitsintensität. Erst für Jungen über 17 Jahre hat die Landgröße einen negativen Einfluss auf die Bildungsbeteiligung. ${ }^{152}$ Zumindest für die älteren Jungen scheint die auf der Farm anfallende Arbeit ein Grund für den Rückzug aus der Schule zu sein. Dieser Zusammenhang zeigt sich auch in der rapiden Senkung der Einschulungsraten für diese Altersgruppe

JACOBY stellte fest, dass ein Familieneinkommen aus selbständiger Arbeit keinen Effekt auf den Bildungserfolg der Kinder hat. Allerdings erhöht das Betriebsvermögen die Wiederholungswahrscheinlichkeit der Kinder. ${ }^{153}$ Auch hier scheint das Betriebsvermögen der Indikator für die vermehrte Arbeitsbelastung

\footnotetext{
148 Vgl. Psacharopoulos/Arriagada (1989), S. 689-695.

149 Vgl. De Vreyer (1993), S. 73.

150 Vgl. Burney/Irfan (1995), S. 28.

151 Vgl. Burney/Irfan (1995), S. 32.

152 Leider waren für die Mädchen dieser Altersgruppe keine Daten verfügbar.

153 Vgl. Jacoby (1994), S. 157.
} 
im Haushalt zu sein. Das daraus resultierende Einkommen behindert den Schulbesuch der Kinder nicht.

LEVISON/MOE berechneten, dass Landbesitz die wöchentliche Arbeitszeit der Mädchen im Haushalt erhöht und belegen damit die höhere Arbeitsbelastung in Farmhaushalten. Allerdings wirkt sich dies nicht auf die Bildungsentscheidungen für die Mädchen aus. Ob dies durch den Status der Familie als Landeigner zu erklären ist, oder die Mädchen weniger stark von den Farmarbeiten betroffen sind, ist nicht zu klären.

Die Untersuchungen zeigen, dass Kinder von selbständigen Haushaltsvorständen und Familien mit Farmbetrieben mit höherer Wahrscheinlichkeit mit Nachteilen für ihr Bildungsniveau rechnen müssen und unterstützen damit die Hypothese, dass die Opportunitätskosten die Bildungsentscheidung negativ beeinflussen.

Nur SKYT NIELSEN ermittelte ein Ergebnis, das diesen Vermutungen widerspricht. Sie berechnete höhere Wahrscheinlichkeiten für den Schulbesuch für Kinder, deren Familien in der Landwirtschaft tätig sind.

Nicht nur in Familienbetrieben fallen Arbeiten an, bei denen die Mithilfe der Kinder üblich ist. Zu nennen ist hier vor allem die Hausarbeit. Da die Hausarbeit in fast allen Entwicklungsländern traditionell Aufgabe der Frauen ist, dürften die dadurch verursachten Opportunitätskosten bei Mädchen eine größere Rolle spielen als bei Jungen. Einige Untersuchungen wählten daher die Mädchen als Zielgruppe oder differenzierten ihre Ergebnisse nach dem Geschlecht der Schüler.

LEVISON/MOE untersuchten in ihrer Studie, ob die traditionell von Mädchen durchgeführte Hausarbeit junge Peruanerinnen in ihrer Bildungsteilnahme behindert. Sie erfragten, wie viele Stunden peruanische Mädchen im Alter von 5 bis 19 Jahren im Durchschnitt im Haushalt halfen. Die wöchentliche Stundenzahl steigt von 5 Stunden bei den 6jährigen auf über 20 Stunden bei den 1819jährigen. ${ }^{154} \mathrm{Da}$ die Autorinnen nur Mädchen als Stichprobe ausgewählt haben, können sie keinen Vergleich der Geschlechter aufstellen.

GERTLER/GLEWWE untersuchten beide Geschlechter. Wie sie ermittelten, reduziert der Schulbesuch die Arbeitszeiten im Haushalt bei den jüngeren Mädchen um 4 Stunden und bei den Älteren um bis zu 16 Stunden wöchentlich. Die Jungen beider Altersgruppen halfen im Schnitt sogar 2,5 Stunden mehr im Haushalt, wenn sie zur Schule gingen. Allerdings muss hier berücksichtigt werden, dass sie ihre Arbeitszeit vor allem außerhalb des eigenen Haushalts leisten. Bei

154 Vgl. Levison/Moe (1998), Fig. 2, S. 343. 
der Gesamtbelastung liegen beide Geschlechter ungefähr gleich auf. Nur ältere Mädchen arbeiten insgesamt etwas mehr, und senken ihre Arbeitszeit durch den Schulbesuch deutlicher als ihre gleichaltrigen Brüder. ${ }^{155}$

Tabelle 6.5: Alter und Geschlecht des Kindes als Bildungsdeterminante

\begin{tabular}{|c|c|c|c|c|c|}
\hline Autor & Land & Zielgr. & abh. Variable & erkl. Variable & Ergebnis \\
\hline \multirow[t]{4}{*}{$\begin{array}{l}\text { Levison/ } \\
\text { Moe, } 1998\end{array}$} & \multirow[t]{4}{*}{$\begin{array}{l}\text { Peru, } \\
\text { ländlich }\end{array}$} & \multirow[t]{4}{*}{$\begin{array}{l}\text { 10-19, nur } \\
\text { Mädchen }\end{array}$} & $\begin{array}{l}\text { Beteiligung an der } \\
\text { Hausarbeit, j/n }\end{array}$ & Alter des Kindes & positiv \\
\hline & & & $\begin{array}{l}\text { wöchentliche Ar- } \\
\text { beitszeit im HH }\end{array}$ & Alter des Kindes & $\begin{array}{l}\text { steigt um } 80 \text { Minu- } \\
\text { ten mit jedem Le- } \\
\text { bensjahr }\end{array}$ \\
\hline & & & $\begin{array}{l}\text { Bildungsbeteili- } \\
\text { gung, j/n }\end{array}$ & Alter des Kindes & negativ \\
\hline & & & $\begin{array}{l}\text { wöchentliche Zeit } \\
\text { in der Schule }\end{array}$ & Alter des Kindes & $\begin{array}{l}\text { sinkt um } 12 \text { Min. } \\
\text { mit jedem LJ }\end{array}$ \\
\hline \multirow{2}{*}{$\begin{array}{l}\text { Gertler/ } \\
\text { Glewwe, } \\
1992 \\
\end{array}$} & \multirow{2}{*}{$\begin{array}{l}\text { Peru, } \\
\text { ländlich }\end{array}$} & \multirow{2}{*}{$\begin{array}{l}\text { 10-18 } \\
\text { Jahre }\end{array}$} & Bildungsteilnahme & Kind ist weiblich & negativ \\
\hline & & & Bildungsteilnahme & Alter des Kindes & negativ \\
\hline
\end{tabular}

LEVISON/MOE ermittelten, dass ältere Mädchen mit einer höheren Wahrscheinlichkeit an der Hausarbeit beteiligt werden. Sie wenden außerdem mit jedem zusätzlichen Lebensjahr ca. 80 Minuten mehr Zeit pro Woche für Hausarbeiten auf. Ältere Mädchen scheinen also für die Hausarbeit wertvoller zu werden, was die Opportunitätskosten ihres Schulbesuchs steigen lässt. Das spiegelt sich auch an ihrer sinkenden Bildungsbeteiligung wider. Sowohl die Wahrscheinlichkeit des Schulbesuchs als auch die wöchentliche Anwesenheit in der Schule sinkt mit dem Alter. ${ }^{156}$

Auch die Nachfrageschätzung von GeRTLER/GLEWWE konnte den, durch die durchschnittliche Arbeitsbelastung erwarteten, Zusammenhang bestätigen. Als Ursache für die negativen Koeffizient für das Geschlecht und das Alter der Schüler können demnach die steigenden Opportunitätskosten betrachtet werden.

Weiterhin wird vermutet, dass die Struktur des Haushaltes mitverantwortlich ist für den Zeitbedarf, den die Hausarbeit fordert. Vor allem wird vermutet, dass die Beaufsichtigung jüngerer Geschwister zu den traditionellen Aufgaben von Mädchen in Entwicklungsländern gehört und damit ihre Opportunitätskosten durch den Schulbesuch erhöht. Die folgende Tabelle fasst die Ergebnisse hierzu zusammen.

155 Vgl. Gertler/Glewwe (1992), S. 175. Wie bei Levison und Moe werden auch hier die Living Standard Survey Daten von 1985-86 herangezogen. Allerdings sind hier nur die ruralen Gebiete einbezogen, was zu leicht abweichenden Zahlen führt.

156 Vgl. auch zu den folgenden Ausführungen Levison/Moe (1998) S. 349-352. 
Tabelle 6.6: Familienstruktur als Bildungsdeterminante

\begin{tabular}{|c|c|c|c|c|c|}
\hline Autor & Land & Zielgruppe & abhängige Variable & erklärende Variable & Ergebnis \\
\hline \multirow[t]{2}{*}{$\begin{array}{l}\text { Jamison/ Lock- } \\
\text { heed, } 1987\end{array}$} & \multirow{2}{*}{$\begin{array}{l}\text { Nepal } \\
\text { ländlich }\end{array}$} & & Teilnahme, j/n & $\begin{array}{l}\text { Mädchen mit Geschwistern kleiner } \\
\text { als } 7 \text { Jahre }\end{array}$ & negativ \\
\hline & & & Teilnahme, j/n & Mädchen ohne Geschwister <7 J. & negativ \\
\hline \multirow{9}{*}{$\begin{array}{l}\text { Levison/Moe, } \\
1998\end{array}$} & \multirow{9}{*}{$\begin{array}{l}\text { Peru, } \\
\text { ländlich }\end{array}$} & \multirow{9}{*}{$\begin{array}{l}\text { 10-19, nur } \\
\text { Mädchen }\end{array}$} & wöchentliche Arbeitszeit im $\mathrm{HH}$ & Anzahl Geschwister $<7$ Jahre & steigt um $0,8 \mathrm{~h}$ \\
\hline & & & wöchentliche Schulzeit & Anzahl Geschwister $<7$ Jahre & sinkt um $0,4 \mathrm{~h}$ \\
\hline & & & wöchentliche Arbeitszeit im $\mathrm{HH}$ & Anzahl Mädchen, 10-19 Jahre & sinkt um 1h \\
\hline & & & wöchentliche Arbeitszeit im HH & Anzahl Frauen, 25-54 Jahre & sinkt um $2 \mathrm{~h}$ \\
\hline & & & wöchentliche Arbeitszeit im HH & Anzahl Jungen, 10-19 Jahre & steigt um $0,5 \mathrm{~h}$ \\
\hline & & & wöchentliche Arbeitszeit im HH & Anzahl Jungen >19 Jahre & steigt um $0,5 \mathrm{~h}$ \\
\hline & & & Bildungsbeteiligung & Anzahl Frauen $>64$ Jahre & positiv \\
\hline & & & Bildungsbeteiligung & Anwesenheit der Mutter & positiv \\
\hline & & & wöchentliche Schulzeit & Anwesenheit der Mutter & sinkt um 10h \\
\hline \multirow[t]{2}{*}{ Jacoby, 1994} & \multirow[t]{2}{*}{ Peru } & \multirow[t]{2}{*}{ 7-12 Jahre } & \multirow{2}{*}{$\begin{array}{l}\text { Wiederholungswahrscheinlichkeit } \\
\text { Wiederholungswahrscheinlichkeit }\end{array}$} & Anzahl Geschwister <7 Jahre & positiv \\
\hline & & & & weibliche ältere Schwester & negativ \\
\hline \multirow{2}{*}{$\begin{array}{l}\text { Psacharopou- } \\
\text { los/Arriagada, } \\
1989\end{array}$} & \multirow[t]{2}{*}{ Brasilien } & \multirow[t]{2}{*}{ 7-14 Jahre } & Teilnahme, j/n & Anwesenheit Geschwister $<7 \mathrm{~J}$. & negativ \\
\hline & & & Erreichungsgrad & Anwesenheit Geschwister < 7 J. & negativ \\
\hline \multirow{3}{*}{$\begin{array}{l}\text { Leibovich/ } \\
\text { Magnac, } 1996\end{array}$} & \multirow{3}{*}{$\begin{array}{l}\text { Kolumbien, } \\
\text { ländlich }\end{array}$} & \multirow[t]{3}{*}{ 5-17 Jahre } & Bildungsteilnahme & Anwesenheit der Mutter & positiv \\
\hline & & & Bildungsteilnahme & Anzahl Frauen 17-40 & positiv \\
\hline & & & Bildungsteilnahme & Anzahl Männer >60 & positiv \\
\hline
\end{tabular}


Zunächst fällt auf, dass alle Untersuchungen der Anwesenheit von Kindern im Vorschulalter einen negativen Effekt auf die Ausbildung der älteren Geschwister nachweisen. Tatsächlich scheint dies vor allem die Mädchen zu betreffen.

Nachdem JAMISON/LOCKHEED ermittelt hatten, dass Jungen im Durchschnitt höhere Bildungserfolge erzielten als Mädchen, fügten sie in ihre Gleichung für die Bildungsteilnahme die zwei o.g. weitere Variablen ein. Damit konnten sie zeigen, dass Mädchen weniger wahrscheinlich zur Schule geschickt werden, und dass ihre Bildungsaussichten zusätzlich noch von der Anwesenheit jüngerer Geschwister verschlechtert werden. ${ }^{157}$

Auch LEVISON/MoE bestätigten, dass die Anwesenheit kleinerer Geschwister die von den Mädchen zu leistende Zeit im Haushalt erhöht. Ihre grundsätzliche Bildungsbeteiligung in der letzten Woche wurde zwar nicht signifikant beeinflusst, aber sie waren im Schnitt weniger häufig anwesend.

Ebenso fanden JACOBY158 und PSACHAROPOULOS/ARRIAGADA heraus, dass das Vorhandensein jüngerer Geschwister einen negativen Einfluss auf den schulischen Erfolg hat. Sowohl die Teilnahmewahrscheinlichkeit als auch der Bildungserfolg wird negativ beeinflusst. Sie differenzieren ihre Berechnungen nicht nach dem Geschlecht.

LEVISON/MOE untersuchten den Einfluss der Familienstruktur noch differenzierter. Die Anwesenheit von Jungen und Männern im Haushalt erhöhen die Zeit, die ein Mädchen im Haushalt hilft. Andere Mädchen zwischen 10 und 19 Jahren und erwachsene Frauen von 25-54 Jahren reduzieren die geleistete Hausarbeit der Mädchen. ${ }^{159}$ Frauen über 64 erhöhen sogar die Wahrscheinlichkeit des Schulbesuchs der Mädchen. Die Aufgaben im Haushalt scheinen zwischen den anwesenden Frauen geteilt zu werden. Das Fehlen eines Schulmädchens scheint durch andere Mädchen und Frauen kompensiert werden zu können. Männer scheinen sich an dieser Aufgabenteilung nicht zu beteiligen. Sie verursachen eher zusätzliche Arbeit für die Mädchen.

LEIBOVICH/MAGNAC und JACOBY bestätigen diese Ergebnisse bezüglich der Anwesenheit von Frauen. LEIBOVICH/MAGNAC scheinen allerdings den Einfluss der Männer zu widerlegen. Allerdings handelt es sich in ihrer Studie um alte Männer

157 Vgl. Jamison/Lockheed (1987), S. 297 und 302.

158 Vgl. Jacoby (1994), S. 157-158.

159 Die Autorinnen ermitteln trotz der entgegen gesetzten Auswirkungen auf die Hausarbeit sowohl für die Anzahl der Brüder als auch der Schwestern im schulpflichtigen Alter einen negativen Effekt auf die Bildungsbeteiligung. Da dieser Effekt unabhängig von der anfallenden Arbeit zu sein scheint, wird es sich dabei um Konkurrenz um knappe Ausbildungsmittel handelt. Diese Zusammenhänge werden weiter unten behandelt. 
über 60 Jahre, die eher im Haushalt anwesend und damit für einige Aufgaben verfügbar sind.

Wenn die Substitutionshypothese der Frauen Gültigkeit besitzt, müsste auch die Anwesenheit der Mutter im Haushalt die Kinder entlasten. LEVISON/MOE und LEIBOVICH/MAGNAC bestätigten einen positiven Effekt der Mutter auf die Bildungsbeteiligung der Kinder. LEVISON/MOE ermittelten aber außerdem das unerwartete Ergebnis, dass ein Mädchen im Durchschnitt 10 Stunden pro Woche weniger zur Schule geht, wenn seine Mutter im Haushalt anwesend ist. Sie geben einen möglichen Erklärungsansatz für ihre Stichprobe, die im ländlichen Peru wohl vornehmlich aus traditionellen Haushalten besteht: Da die Mädchen bezüglich ihrer Haushaltspflichten eine Art Lehre bei der eigenen Mutter absolvieren, bestehen ihre Opportunitätskosten des Schulbesuchs auch in der verlorenen Trainingszeit mit der Mutter. Ihre Ergebnisse würden dann Hinweise darauf geben, dass dieses Training von der Anwesenheit der Mutter abhängt und unabhängig von der Einschulung des Kindes fortgeführt wird, was seine Anwesenheit in der Schule negativ beeinflusst. ${ }^{160}$ Diese Interpretation besagt, dass auch der familieninternen (informellen) Ausbildung der Kinder ein Wert beigemessen wird, der mit der formalen Schulausbildung konkurriert.

Als weiterer Indikator für die Höhe der Opportunitätskosten im Haushalt können Besitztümer bezeichnet werden, die die Arbeitsbelastung im Haushalt senken. Da in keiner Studie die Auswirkungen von Waschmaschinen, Kühlschränken u. ä. auf die Ausbildung der Kinder untersucht wurde, sollen hier nur kurz auf die folgende Tabelle eingegangen werden.

Tabelle 6.7: Besitztümer als Bildungsdeterminante

\begin{tabular}{|c|c|c|c|c|c|}
\hline Autor & Land & Zielgr. & abhängige Variable & erkl. Var. & Ergebnis \\
\hline \multirow{7}{*}{$\begin{array}{l}\text { Levison/ } \\
\text { Moe, } 1998\end{array}$} & \multirow{7}{*}{$\begin{array}{l}\text { Peru, } \\
\text { ländlich }\end{array}$} & \multirow{7}{*}{$\begin{array}{l}\text { 10-19, nur } \\
\text { Mädchen }\end{array}$} & Beteiligung an Hausarbeit, j/n & Wasser & negativ \\
\hline & & & Beteiligung an Hausarbeit, j/n & Telefon & negativ \\
\hline & & & wöchentl. Arbeitszeit im HH & Wasser & sinkt um 1,6h \\
\hline & & & wöchentl. Arbeitszeit im HH & Telefon & sinkt um 2,3h \\
\hline & & & Bildungsteilnahme, j/n & Wasser & positiv \\
\hline & & & Bildungsteilnahme, j/n & Telefon & positiv \\
\hline & & & wöchentl. Zeit in der Schule & Wasser & steigt um $1,3 \mathrm{~h}$ \\
\hline \multirow{4}{*}{$\begin{array}{l}\text { Psacharo- } \\
\text { poulos/ } \\
\text { Arriagada, } \\
1989\end{array}$} & \multirow[t]{4}{*}{ Brasilien } & \multirow[t]{4}{*}{ 7-14 Jahre } & Teilnahme, j/n & Wasser & positiv \\
\hline & & & Teilnahme, j/n & Toilette & positiv \\
\hline & & & Erreichungsgrad & Wasser & positiv \\
\hline & & & Erreichungsgrad & Toilette & positiv \\
\hline
\end{tabular}

PSACHAROPOULOS/ARRIAGADA konnten dem Vorhandensein von Wasserleitungen und Toilettenanlagen im Haushalt eine positiv Wirkung auf die Ausbildung der Kinder nachweisen. Sie erklären diesen Zusammenhang allerdings mit dem positiven Zusammenhang zwischen dem Anschluss einer Gemeinde an ein 
Wasser- und Abwassersystem und dem Vorhandensein von Schulen. ${ }^{161} \mathrm{Da}$ in den Schätzungen, die das Vorhandensein von Schulen in ihre Untersuchung integriert haben, kein Effekt auf die Ausbildung nachgewiesen werden konnte, ist diese Interpretation anzuzweifeln. Ebenso könnten diese Variablen auch als Wohlstandsindikator dienen. Ob Wasser- und Abwassersysteme sich tatsächlich arbeitserleichternd auswirken und damit die Opportunitätskosten des Schulbesuchs der Kinder senken, kann nur vermutet werden.

LEVISON/MOE untersuchten diesen Zusammenhang genauer. Sie schätzten den Einfluss von fließend Wasser und Telefon im Haushalt auf die von Mädchen im Haushalt geleisteten Arbeitsstunden. Beide Variablen senkten sowohl die Wahrscheinlichkeit der Mädchen, sich überhaupt an der Hausarbeit zu beteiligen, und sie senkten die durchschnittlich im Haushalt geleistete Arbeitszeit deutlich. Da sich beide Variablen auch positiv auf die Bildungsbeteiligung auswirkten, liegt der Schluss nahe, dass der Zusammenhang durch die gesunkenen Opportunitätskosten erklärt werden kann.

Wie die Auswertungen zeigen, lassen sich auch die Opportunitätskosten innerhalb der Familie nicht direkt messen. Allerdings zeigen die Ergebnisse zu einigen Hilfsindikatoren ein recht einheitliches Bild. Die Notwendigkeit der Mithilfe im eigenen Haushalt erhöht die Opportunitätskosten der Ausbildung und senkt die Bildungsbeteiligung.

\subsubsection{Zusammenfassung der Opportunitätskosten}

Die obigen Auswertungen zeigen, dass die Opportunitätskosten eine große Rolle bei der Bildungsentscheidung in Entwicklungsländern zu spielen scheinen.

Die Vermutung liegt nahe, dass die fehlende Mithilfe im Haushalt insbesondere arme Familien betrifft, da die Hausarbeit hier extrem zeitintensiv sein kann. Wenn z.B. kein fließend Wasser, kein Kühlschrank und keine Waschmaschine vorhanden ist, muss Wasser geholt, häufig eingekauft und zeitintensiv gewaschen werden. Kleinere Kinder brauchen rund um die Uhr Betreuung. Da in Lateinamerika in der Regel schon die Mittelschicht Hausangestellte beschäftigt, und die Ausstattung mit technischen Geräten mit dem Einkommen steigt, ist die zeitintensive Haushaltsführung eher ein Problem der unteren Einkommensgruppen.

Nicht nur, dass arme Familien oft Merkmale aufweisen, die hohe Opportunitätskosten generieren, auch die Knappheit an finanziellen Ressourcen selber erhöht den Wert der Zeit der Kinder. Wenn die Arbeit der Kinder nicht durch die Einstellung von Arbeitskräften kompensiert werden kann, oder zur Einstellung

161 Vgl. Psacharopoulos/Arriagada (1989), S. 689. 
einer Arbeitskraft Geld zu ungünstigen Konditionen geliehen werden muss, wird der Schulbesuch teurer und das Kind bricht seine Ausbildung früher ab. Der Einfluss der Opportunitätskosten ist in kreditrationierten Haushalten größer als in Haushalten mit Zugang zum Kapitalmarkt.

Auch unter sonst gleichen Bedingungen in den Haushalten, haben die Opportunitätskosten für arme Familien mehr Relevanz bei der Bildungsentscheidung für die Kinder. Dieser Zusammenhang wird besonders deutlich, wenn die Kinder durch ihren Schulbesuch auf eigenes Einkommen verzichten müssen. Eine Familie, die am oder unterhalb des Existenzminimums lebt, kann sich diesen Verzicht nicht leisten. Und selbst kurz oberhalb des Existenzminimums ist der marginale Nutzen jedes zusätzlichen Einkommens sehr hoch. ${ }^{162}$ Deshalb werden die Opportunitätskosten im Verhältnis zu Einkommen gemessen.

Obwohl zu vermuten ist, dass besonders arme Familien besonders stark von den Opportunitätskosten beeinflusst werden, wurden in keiner der untersuchten Studien die Ergebnisse nach Einkommensgruppen differenziert. 


\subsection{Nutzenfaktoren als Bildungsdeterminante}

Wie die Ausführungen in Kapitel 2.1.2 gezeigt haben, sind die Nutzen der Ausbildung breiter gefächert als die Kosten. Wird der Investitionsansatz als realistisch betrachtet, liegt der größte und eindeutigste Effekt der Ausbildung in der dadurch induzierten Einkommenssteigerung. Da es sich hierbei um eine zukünftige Größe handelt, ist ihr exakter Wert den Familien nicht bekannt. Sie bilden Erwartungen über diese Größe, die bei der Bildungsentscheidung eine große Rolle spielen müssten. Soziale Studien über das Verhalten von Eltern und Schülern haben gezeigt, dass die Berufsaussichten unter den verschiedenen Motivationen, in weitere Ausbildung zu investieren, ganz oben rangieren. ${ }^{163}$ Dennoch hat keiner der oben genannten Autoren die erwarteten Einkommen in die Nachfrageschätzungen integriert. Dies kann nur durch den Mangel an adäquaten Daten zu den Erwartungen erklärt werden. Da Erwartungen subjektiver Art und nicht beobachtbar sind, müssen sie explizit erfragt werden. Sie sind nicht üblicherweise Bestandteil von Haushaltsdatensätzen.

\subsubsection{Erwartete Nutzen der Ausbildung}

Nur wenige Autoren integrierten eine Ersatzvariable, um dennoch Aufschlüsse über den erwarteten Output von Bildung zu erhalten.

BEDI/MARSHALL schätzten den Effekt von Schulcharakteristika auf die Ergebnisse von Mathematik- und Spanischprüfungen, um ein Maß für den Output zu erhalten. Anhand dieser Bildungsproduktionsfunktion konnten sie Testergebnisse für jedes Individuum voraussagen. Diese Werte integrierten sie in die Schätzung der Bildungsbeteiligung. ${ }^{164}$ Die Ergebnisse bestätigen ihre Hypothese, dass die erwarteten Ergebnisse eine wichtige Rolle in der elterlichen Entscheidung über die Bildungsbeteiligung der Kinder spielen. Die Erhöhung der durchschnittlichen Leistung um fünf Punkte erhöht die Bildungsbeteiligung um 3 bis 7 Tage pro Schuljahr. ${ }^{165}$ Ob mit diesen besseren Leistungen höhere Einkommen assoziiert werden, kann nur vermutet werden. Ebenso kann die gute Leistung an sich einen eigenen Wert oder einen Motivationsschub darstellen.

GLEWWE/GROSH/JACOBY/LockHEED stellten fest, dass obwohl für Mädchen bessere Testergebnisse im Lesen und Schreiben zu erwarten sind, sie nicht häufiger auf die akademische Variante der Grundschulen in Jamaika geschickt wer-

\footnotetext{
163 Vgl. Blaug (1991), S. 170 und Hicks (1996), S. 6.

164 Zum Vorgehen und den notwendigen ökonometrischen Anpassungen des Modells vgl. Bedi/Marshall (1999), S. 658-660.

165 Vgl. Bedi/Marshall (1999), S. 669.
} 
den. ${ }^{166}$ Hier reichen die schulischen Leistungen nicht aus, um die Bildungsinvestition zu erhöhen. Dies kann daran liegen, dass die Verdienstaussichten für Mädchen unabhängig vom Leistungs- bzw. Bildungsniveau pessimistisch eingeschätzt werden. ${ }^{167}$ Ebenso gut kann die traditionelle Frauenrolle dazu führen, dass für Frauen keine höhere Bildung nachgefragt wird, ohne dass die Leistungen oder die zukünftigen Möglichkeiten am Arbeitsmarkt überhaupt im Kalkül berücksichtigt werden.

GERTLER/GLEWWE finden heraus, dass für Mädchen auf dem Land kein Einkommensanstieg durch den Besuch der Sekundaria zu erwarten ist. ${ }^{168}$ Dennoch ermitteln die Autoren eine Bereitschaft der Eltern, für die Sekundarbildung der Mädchen zu bezahlen. Sie bewerten die Ausbildung der Mädchen nur wenig schlechter als für Jungen, obwohl der ökonomische Wert am Arbeitsmarkt zweifelhaft ist. Entweder die Eltern schätzen die Ertragsaussichten für Mädchen nicht richtig ein, oder die Ausbildung muss neben den erwarteten Einkommenssteigerungen weitere Nutzen aufweisen. Die Autoren geben einige mögliche Erklärungen. Erstens, die Sekundaria könnte positive Auswirkungen auf die Arbeit der Mädchen im Haushalt haben. Zweitens, einige Eltern könnten erwarten, dass ihre Töchter in die Städte migrieren, wo sich die Sekundarausbildung auf die Löhne auswirkt. Drittens, falls die Erträge einer höheren Ausbildung für Mädchen hoch sind, könnten die Eltern die Sekundaria als notwendige Voraussetzung für weitere Bildung betrachten. Und viertens, könnten besser ausgebildete Mädchen ganz einfach einen eigenen Wert für einige Eltern haben. ${ }^{169}$

MCMAHON fragte die Schüler nach einem Maß für die Unsicherheit über ihre zukünftigen Einkommen. Wenn dieses Maß in die Nachfragefunktion integriert wird, zeigt sich zwar ein negativer Einfluss größerer Unsicherheit, der Faktor stellt aber keine signifikante Einflussgröße dar. Der Autor ermittelt außerdem eine positive Korrelation zwischen der Unsicherheit und den Fähigkeiten der Kinder, was er durch die Antizipation einer breiteren Menge an Möglichkeiten

166 Vgl. Glewwe/Grosh/Jacoby/Lockheed (1995), S. 244-245. Während die Primaria meistens mit der Sekundaria fortgesetzt wird, werden Schüler der All-Age-Schools häufiger direkt in den Arbeitsmarkt entlassen.

167 Das heißt, es werden keine Einkommenssteigerungen erwartet, die das Kalkül zugunsten von mehr Ausbildung verschieben würden.

168 Vgl. Gertler/Glewwe (1992), S. 175-177. Sie beziehen sich dabei auf Untersuchungen von Stelcner/Arriagada/Moock (1987) und King (1989) über den Einfluss der Bildung auf die Löhne, auf Moock/Musgrove/Stelcner (1989) über den Einfluss der Bildung auf die Einkommen von selbständigen Unternehmern und auf Jacoby (1989) über den Einfluss der Bildung auf landwirtschaftliche Einkommen.

169 Vgl. Gertler/Glewwe (1992), S. 186. 
erklärt. ${ }^{170}$ In einer anderen Untersuchung stellte er fest, dass Frauen sich unsicherer über ihre zukünftigen Einkommen äußern. ${ }^{171}$

WHITFIELD/WILSON präsentierten eine Zeitreihenanalyse über die Faktoren, die die Bildungsteilnahme englischer und walisischer Kinder über die Schulpflicht hinaus beeinflussen. In ihrer Makro-Analyse ermittelten sie einen positiven Zusammenhang zwischen der aggregierten Bildungsbeteiligung und der durchschnittlichen Ertragsrate der Bildung mit einer Elastizität von ca. 0,3. Die dazu integrierte Ertragsrate berechneten sie aus den entgangenen Einkommen und den Einkommenszuwächsen unter Berücksichtigung der Daten zahlreicher Querschnittanalysen zu erwarteten Einkommensprofilen bei alternativen Bildungsniveaus. ${ }^{172}$

\subsubsection{Erwartete Bildungserträge - Allgemeine Erkenntnisse}

Obwohl die Ertragserwartungen ein zentrales Element der Humankapitaltheorie sind, wurde ihr Einfluss auf die Bildungsentscheidung bisher nicht untersucht. Einige wenige Wissenschaftler haben diesen Mangel kritisiert und Befragungen zu den Erwartungen durchgeführt. Obwohl nicht empirisch nachgewiesen ist, ob sich diese Erwartungen tatsächlich in höheren Nachfragemengen ausdrücken, zeigen die Untersuchungen, ob mit höheren Bildungsniveaus überhaupt Einkommenssteigerungen erwartet werden.

Als zwei der ersten Autoren führten McMAHON/WAGNER 1971/72 in den USA eine Studie durch, bei der sie 2.766 Erstsemester nach ihren erwarteten Einkommen nach Abschluss ihrer Ausbildung und 25 Jahre nach Eintritt in den Arbeitsmarkt befragten. Diese Angaben verglichen sie dann mit den durchschnittlichen Einstiegsgehältern in den entsprechenden Abschlussjahren. Die Ergebnisse wurden 1981 veröffentlicht. ${ }^{173}$

Nach Geschlecht und Hautfarbe differenziert ergaben sich die folgenden Muster: Frauen erwarten etwas niedrigere Einstiegsgehälter als Männer und rechnen auch mit einer niedrigeren Wachstumsrate über ihr Erwerbsleben. ${ }^{174}$ Schwarze Schüler hingegen erwarten etwa die gleichen Einstiegsgehälter und Steigerungsraten wie ihre weißen Mitschüler. Diejenigen, die höhere Abschlüsse anstreben, erwarten sogar leicht höhere Einkommen als die Weißen. Nach dem geplanten Abschluss differenziert zeigt sich, dass die erwarteten Ein-

170 Vgl. McMahon (1984), S. 86.

171 Vgl. McMahon (1987), S. 196.

172 Vgl. Whitfield/Wilson (1991), S. 399 - 402.

173 Vgl. McMahon/Wagner (1981), S. 274-285.

174 Unterschiedliche Erwartungen bei Frauen und Männern untersuchten später auch Blau und Ferber bei College Business School Seniors in den USA. Vgl. Blau/Ferber (1991), S. 581-607. 
stiegsgehälter mit dem angestrebten Abschluss nur leicht steigen. Zum Beispiel steigt für Jungen das erwartete Einstiegsgehalt von $\$ 10.087$ mit einem Bachelor's Degree auf nur \$11.244 mit einem Doktortitel. Allerdings erwarten die Jungen, die mit dem Bachelor's Degree die Schule verlassen wollen, nur eine durchschnittliche Wachstumsrate von 3,56\% pro Jahr, während die angehenden Doktoren mit einem sehr viel steileren Anstieg ihrer Gehälter rechnen (8,92\%). Daraus ergibt sich, dass die erwarteten Einkommensunterschiede über die Zeit wachsen. Schüler, die einen Bachelor's Degree anstreben, rechnen in 25 Jahren mit einem Anstieg um ca. \$8.000, Anwärter eines Master's Degree erwarten einen Anstieg um \$10.000 und Doktoranwärter erwarten sogar einen Anstieg um \$17.000. Sowohl die höheren erwarteten Einstiegsgehälter als auch der schnellere angenommene Anstieg derselben waren konsistent mit den entsprechenden beobachtbaren tatsächlichen Alters-Einkommens-Profilen bei verschiedenen Bildungsniveaus. Auch nach Beschäftigungsfeldern differenziert, ergibt sich eine deutliche Übereinstimmung der erwarteten Einstiegsgehälter mit den entsprechenden tatsächlichen Gehaltsofferten am Arbeitsmarkt.

Die Ergebnisse zeigen also, dass die Schüler höherer Bildungsstufen in den USA die Erträge zusätzlicher Ausbildungsinvestitionen ziemlich korrekt antizipierten. Allerdings steigt der Variationskoeffizient der Angaben mit der zeitlichen Entfernung des zu schätzenden Einkommens und mit der Höhe des Bildungsniveaus.

Auch Dominitz/MANSKI befragten 1992 High-School Schüler und College Undergraduates nach ihren Einkommenserwartungen. Da diese Erwartungen einen Schlüsselfaktor in der ökonomischen Theorie für das Bildungsverhalten darstellen, erwarteten die Autoren, auf eine substantielle Literatur zur Erhebung und Analyse von Erwartungsdaten zurückgreifen zu können. Allerdings fanden sie kaum Literatur. Einzelne Studien fragten zwar nach zukünftigen Einkommenserwartungen, setzten diese aber nicht mit dem erreichten Ausbildungsniveau oder nicht einmal mit der befragten Person in Beziehung. Einige sozialpsychologische Studien fragten zwar nach dem erwarteten Einfluss der Ausbildung auf den Erfolg am Arbeitsmarkt, formulierten ihre Fragen aber zu vage, um für ökonomische Analysen wertvoll zu sein. ${ }^{175}$ Sie mussten daher nahezu Neuland betreten, was dazu führte, dass ihr Beitrag mehr Einsichten in das Design und die Durchführung von Erwartungsstudien als in die Erwartungsstruktur von Schülern gibt. Ihre relativ kleine Stichprobe enthält vor allem Kinder von Eltern mit einer College Ausbildung und einem Einkommen über dem US-

175 Vgl. Dominitz/Manski (1996), S. 1-4. Zu der Verwertbarkeit von psychologischen und soziologischen Studien vgl. auch Manski (1993), S.46-47. 
amerikanischen Durchschnitt. Für diese Stichprobe ermittelten sie folgende Zusammenhänge: Trotz hoher Unterschiede in den Einkommenserwartungen und in den erwarteten Einkommensverteilungen innerhalb der Gruppen (HighSchool/College, Female/Male), existiert die allgemeine Meinung, dass die College Ausbildung positive Erträge bringt, und dass die Einkommen zwischen dem 30sten und 40sten Lebensjahr steigen. Außerdem werden die eigenen Einkommen als eher unsicher betrachtet und die Ungleichverteilung der Einkommen in der amerikanischen Gesellschaft überschätzt. ${ }^{176}$ Zusammenfassend weisen die Autoren allerdings auf die Notwendigkeit weiterer Forschung hin. Unter anderem sollte untersucht werden, ob Jugendliche aus weniger privilegierten Schichten ebenso auswertbare Antworten auf Wahrscheinlichkeitsbefragungen geben können, und mit größeren Stichproben sollten die Erwartungen verschiedener Bevölkerungsgruppen untersucht werden.

Eine Befragung über die Einkommenserwartungen von Undergraduates an einem öffentlichen Four-Year College in Kalifornien in 1992/1993 wurde von BETTS ausführlich ausgewertet. Die Studie gibt interessante Einblicke in den Erwartungsbildungsprozess. ${ }^{177}$

Die Studenten wurden zu ihren Erwartungen bezüglich der aktuellen Einkommen von jungen (25-34) Arbeitnehmern mit High School und Bachelor's Degree unabhängig von ihrem Betätigungsfeld gefragt. Außerdem sollten sie schätzen, welche Einstiegsgehälter junge Berufsanfänger mit unterschiedlichen Bildungsniveaus in verschiedenen Branchen verdienen. Für Ingenieure werden diese Fragen noch um verschiedene Jahre an Arbeitserfahrung erweitert. Die Befragungsergebnisse zeigen, dass bei höherem Bildungsniveau höhere Einstiegsgehälter erwartet werden und dass die Einkommen mit den Jahren an Berufserfahrung steigen. Die mittleren Erwartungen stimmen erstaunlich mit den tatsächlichen Einkommen überein, zeigen aber vor allem am oberen Ende des Erfahrungsprofils eine große Variation der Erwartungen. Die Schätzungen der Gehälter von jungen Arbeitnehmern sind also ziemlich gut, werden aber für Verdiener höheren Alters schlechter. Insgesamt unterschätzen sie den Anstieg der Gehälter.

Um zu testen, ob es systematische Zusammenhänge zwischen den Erwartungen und den Merkmalen der Studenten gibt, führte BETTS eine Regression für die Einkommenserwartungen durch. Dabei wurden neben dem besuchten Studienjahr auch persönliche Charakteristika und Variablen für den familiären Hintergrund integriert. Weder die Variable für die Fähigkeiten der Schüler noch ob

176 Vgl. Dominitz/Manski (1996), S. 25.

177 Vgl. Betts (1996), S. 27-56. 
die Eltern ein College besucht haben spielt eine signifikante Rolle in der Erwartungsbildung. Interessant ist allerdings, dass Studenten im vierten Jahr signifikant niedrigere Einkommenserwartungen haben als Erstsemester. Für die Einkommen von College Absolventen und die verschiedenen Einstiegsgehälter trifft dies auch für Studenten aus Haushalten mit niedrigem Einkommen zu. Sie haben hier signifikant niedrigere Erwartungen als ihre Kommilitonen aus wohlhabenderen Familien.

Außerdem schätzen Studenten die Einstiegsgehälter im angestrebten Berufsfeld signifikant höher ein als anders spezialisierte Kommilitonen. Hier stellt sich nun die Frage, ob die Studenten in ihrem ausgewählten Bereich besser informiert sind, oder ob sie diesen Bereich gewählt haben, weil sie die Einkommen überschätzen. Um diese Frage zu beantworten, schätzte BETTS außerdem noch Abweichung der erwarteten Einkommen von den tatsächlichen Einkommen.178 Dabei fand er heraus, dass Studenten einer gegebenen Fachrichtung signifikant kleinere Fehler bei der Erwartung der Einkommen in Jobs dieser Fachrichtung machen als Studenten anderer Fachrichtungen. Studenten scheinen die Einkommen außerhalb ihrer Fachrichtung also zu unterschätzen, was einen Hinweis darauf gibt, dass sie ihre Informationsbeschaffung auf den ausgewählten Bereich zu spezialisieren scheinen.

Außerdem sinkt die Fehleinschätzung der Einkommen mit der Anzahl der absolvierten Studienjahre. Bei den Einstiegsgehältern wird der Lerneffekt mit zunehmenden Studienjahren besonders deutlich. Die absoluten Fehler sinken monoton mit jedem Jahr und sind in jedem weiteren Jahr signifikant niedriger als im ersten. Der größte Lerneffekt über die Einkommen wird demnach im letzten Jahr erworben. Das spricht dafür, dass sich die Studenten erst dann genauer über den Arbeitsmarkt informieren, wenn sie sich ihren Neigungen und Spezialisierungswünschen bewusst sind. Dieses Ergebnis ist erstaunlich, da Informationen zu Beginn des Studiums am wertvollsten sind, da ein späterer Wechsel der Fachrichtung mit hohen versunkenen Kosten verbunden ist.

Ein weiteres interessantes Ergebnis der Schätzung der Abweichungen ist, dass Kinder aus ärmeren Haushalten signifikant größere Fehler bei der Schätzung der Einkommen machen. Im Zusammenhang mit den Ergebnissen der Erwartungsschätzung zeigt dieses Ergebnis, dass Kinder armer Haushalte die Einkommen von College Absolventen unterschätzen. Hierfür lassen sich verschiedene Erklärungen liefern. Erstens könnten mit höherem Einkommen einfach bessere Informationen gekauft werden. Zweitens könnten niedrige Einkommen auf weniger Familienmitglieder im Arbeitsmarkt schließen lassen. Wenn arbei-

178 Die abhängige Variable ist der logarithmierte absolute Wert der prozentualen Abweichung. 
tende Eltern die Informationsquellen darstellen, sind Kinder armer Familien weniger gut informiert. Drittens: Falls arme Familien geographisch getrennt von reichen Familien leben und Kinder ihre Erwartungen aus den Erfahrungen in ihrer Umgebung bilden, werden sie die Einkommen unterschätzen.

In einer britischen Studie von HICKS von 1994 wurden Schüler nach ihren Hoffnungen für die Zukunft befragt. Die Hoffnung auf einen guten Job rangierte für die Schüler ab 13 Jahren auf dem ersten Platz. ${ }^{179}$ Dieses Ergebnis zeigt, wie wichtig den Schülern ihr beruflicher Werdegang ist. Wenn sie einen positiven Zusammenhang zwischen Ausbildung und Chancen am Arbeitsmarkt erwarten, ist auch anzunehmen, dass ihre Bildungsentscheidung davon gelenkt wird.

Zusammenfassend lässt sich feststellen, dass Studenten in den USA ein relativ realistisches Bild von der Arbeitsmarktsituation haben, obwohl ihre Erwartungen weit streuen und systematisch mit persönlichen Merkmalen variieren. Ihre Informationen über die Einstiegsgehälter (zeitnahe Erwartungen) sind am realistischsten. Außerdem scheinen sie ihre Informationen über den Arbeitsmarkt vor allem in höheren Semestern zu sammeln und sich auf ihre Fachrichtungen zu spezialisieren.

\subsection{Kapitalmarktrestriktionen als Bildungsdeterminante}

In der Theorie bilden die Finanzierungskosten den zweiten großen Einflussfaktor der „Bildungsnachfrage“, da im optimalen Bildungsniveau die Ertragsrate den marginalen Finanzierungskosten angepasst wird. Wenn die Kapitalmärkte perfekt wären, könnte die Nutzenmaximierung der Haushalte in zwei Stufen erfolgen. In einem ersten Schritt wird die Zeit so auf Arbeit und Ausbildung aufgeteilt, dass der Gegenwartswert des Vermögens maximiert wird. In einem zweiten Schritt wird der Konsumpfad gewählt, der den Nutzen des Haushaltes bei gegebenem Vermögen optimiert. Diese Zweiteilung der Entscheidung ist als Separation Theorem bekannt und führt dazu, dass das Ausgangsvermögen zwar den Konsum und die Kreditaufnahme beeinflusst, die „Bildungsnachfrage“ aber unberührt lässt. ${ }^{180}$

Vor allem für Bildungskredite kann der Kapitalmarkt aber als stark rationiert angenommen werden. Wie oben dargestellt wurde müsste nach der Theorie, unter sonst gleichen Bedingungen, ein erschwerter Zugang zum Kapitalmarkt ${ }^{181}$ das optimale Bildungsniveau senken. Die Kapitalmarktbedingungen müssten theo-

179 Vgl. Hicks (1996), S. 6.

180 Vgl. Kodde/Ritzen (1985), S.348 und 354.

181 Durch Zinssteigerungen oder Kreditrationierungen wird die Finanzierungskurve nach oben verschoben und verschiebt damit den Investitionspunkt auf der Ertragsratenkurve nach links. 
retisch die Bildungsnachfrage beeinflussen. Dennoch haben nur wenige Autoren die Wirkungen von Kapitalmarktrestriktionen auf die Bildungsentscheidung empirisch untersucht.

JACOBY untersuchte gezielt die Wirkung von Kapitalmarktbarrieren auf die Bildungserfolge von peruanischen Grundschülern. Dazu unterteilte er seine Stichprobe nach der Wahrscheinlichkeit einer Kreditrationierung zu unterliegen. ${ }^{182} \mathrm{Er}$ ermittelte eine mehr als doppelt so hohe Wiederholerrate für die beschränkten Gruppe. Anschließend wurde die Analyse der Bildungsdeterminanten für beide Gruppen getrennt durchgeführt. Der negative Koeffizient für die Gebrauchsgüter (als Wohlstandsindikator) ist in der kreditbeschränkten Gruppe fast zwei Standardabweichungen größer im Ausmaß, und in der unbeschränkten Gruppe nicht einmal statistisch signifikant. Die Einkommensvariable hat ebenfalls nur auf die beschränkte Gruppe einen signifikanten negativen Einfluss auf die Rückfallwahrscheinlichkeit. Der Koeffizient ist sogar um drei Standardabweichungen größer. ${ }^{183}$ Niedriges Familieneinkommen und geringer Besitz von Gebrauchsgütern senkt den Bildungserfolg der Kinder also nicht, wenn die Familien Zugang zu Krediten haben. In Familien, deren Zugang zu Krediten beschränkt ist, haben diese Variablen allerdings einen signifikanten Einfluss auf den Bildungserfolg der Kinder. Damit zeigte JACOBY, dass „... a separation of human capital investment and consumption decisions exists only in the absence of borrowing constraints"184. Außerdem zeigen die Berechnungen, dass die Opportunitätskosten bei der Bildungsentscheidung eine größere Rolle spielen, wenn der Zugang zum Kapitalmarkt beschränkt ist. ${ }^{185}$ Sowohl das Betriebsvermögen als auch das Vorhandensein jüngerer Geschwister hat nur in der kreditbeschränkten Gruppe einen signifikant positiven Einfluss auf die Rückfallwahrscheinlichkeit. Familien mit Zugang zum Kapitalmarkt scheinen das Fehlen der Schulkinder besser kompensieren zu können. Dieser Zusammenhang spricht auch gegen die These, dass Kinder reicher Eltern nur deshalb seltener Schuljahre wiederholen, weil sie höher motiviert sind oder weil ihre Eltern der Ausbildung mehr Wert beimessen. Mindestens ein Teil der Differenz liegt in der Kapitalknappheit begründet.

SKYT NIELSEN berücksichtigte in ihrer Nachfrageschätzung für Sambia den Zugang zu Krediten. Dazu fügte sie eine Dummy-Variable ein, die den Wert eins

182 Keine Kreditrationierung liegt vor, wenn die Haushalte entweder positive Ersparnisse oder ausstehende Kredite aufweisen. Die Wahrscheinlichkeiten wurden mit einer Probit-Analyse ermittelt und die Stichprobe nach einer Wahrscheinlichkeit über oder unter 0,5 aufgeteilt. Nach dieser Definition galt fast ein Drittel der Kinder als unbeschränkt. Vgl. Jacoby (1994), S. 158.

183 Vgl. Jacoby (1994), S. 158-159.

184 Jacoby (1994), S. 160.

185 Vgl. Jacoby (1994), S. 159. 
annimmt, wenn in der Gemeinde irgendeine Art von Kredit zur Verfügung steht. In diesem Sinne hatten $66 \%$ der Stichprobe Zugang zu Krediten. ${ }^{186}$ In ihrer Schätzung der Bildungsbeteiligung ermittelte sie allerdings negative Koeffizienten für die Variable. Das heißt, dass auch wenn die Familien Zugang zu Krediten haben könnten, sie ihre Kinder lieber nicht in die Schule schicken. Der Grund dafür kann natürlich sein, dass die Kapitalkosten so hoch sind, dass sich eine Investition in Ausbildung nicht lohnt, weil hier niedrigere Erträge erwartet werden. ${ }^{187}$ Andererseits beschreibt ihre Kreditvariable die Gemeinde und nicht direkt den Haushalt. Auch wenn in einer Gemeinde grundsätzlich der Zugang zu Krediten vorhanden ist, heißt das nicht, dass alle Haushalte auch einen Bildungskredit bekommen würden. Da Bildungsinvestitionen keine bankübliche Sicherheit bieten, da sie in den Menschen gebunden sind und nicht veräußert werden können, scheint auch diese Erklärung durchaus realistisch.

LAZEAR entwickelte ein Modell mit dem untersucht werden kann, ob Familien aus unterschiedlichen Einkommensgruppen auch unterschiedlichen Kapitalkosten ausgesetzt sind, wenn sie die Bildungsinvestitionen für ihre Kinder finanzieren wollen. ${ }^{188}$ Er konstruiert zunächst ein Modell mit dem für jedes Individuum die Kosten und Erträge der Ausbildung ermittelt werden kann unter der Annahme, dass die Finanzierungskosten nicht variieren. Damit errechnet er unter Berücksichtigung der aus der Theorie abgeleiteten Background Variablen für jedes Individuum sein Vermögen maximierendes Bildungsniveau. Dabei wurde das Einkommen der Familien als direkter Einflussfaktor nicht berücksichtigt. Außerdem wurde ein für alle gleicher Zinssatz angenommen. Wenn nun Kinder reicher Eltern ihre Ausbildung zu niedrigeren Kosten finanzieren können als Kinder armer Familien, müsste das Modell die optimalen Bildungsniveaus für erstere unterschätzen und für letztere überschätzen. Ein Vergleich der vorausgesagten optimalen mit den beobachteten tatsächlichen Bildungsniveaus belegt diese Vermutung. Um herauszufinden, wie hoch der Zinsunterschied ist, werden unterschiedliche Kostenfunktionen für arme und reiche Haushalte ermittelt und damit ihre jeweils optimalen Bildungsniveaus berechnet. Durch den Einsatz verschiedener Zinsdifferenzen189 kann ermittelt werden, bei welcher Differenz die tatsächlichen Werte am nächsten an die vorhergesagten herankommen. Sein

\footnotetext{
186 Vgl. Skyt Nielsen (1998), S. 8.

187 Die Autorin bezeichnet es als eine Anleihe am Humankapitalmarkt, wenn die Kinder nicht in die Schule geschickt werden, und zieht aus den Ergebnissen den Schluss, dass eine Anleihe am Humankapitalmarkt billiger sein muss als am Kapitalmarkt. Vgl. Skyt Nielsen (1998), S. 16.

188 Vgl. Lazear (1980), S. 42-51.

189 Die Kosten bestehen vor allem aus entgangenen Einkommen, deren Gegenwartswert mit einem für alle gleichen Diskontfaktor ermittelt wurde. Weicht der Leihzins für arme Haushalte nun vom Diskontfaktor (Anlagezins) ab, muss dieser in die Kostenfunktion integriert werden. Bleibt der Leihzins für die Reichen gleich dem Ablagezins, bleibt die Kostenfunktion für diese Bevölkerungsgruppe unverändert.
} 
Ergebnis ist, dass mit 95\%iger Sicherheit die armen Haushalte höheren Finanzierungskosten ausgesetzt sind als die Reichen. Allerdings beträgt der Unterschied weniger als ein viertel Prozentpunkt. ${ }^{190}$

\subsection{Zusammenfassung der Nutzen- und Kostenfaktoren}

Zusammenfassend kann hier gesagt werden, dass für die Kosten- und Nutzenfaktoren der "Bildungsnachfrage“ in Entwicklungsländern in der vorliegenden Literatur kaum Variablen gefunden wurden, die den Zusammenhang eindeutig erklären können. Meistens konnten nur Hilfsindikatoren beobachtet werden, die Hinweise auf den vermuteten Zusammenhang geben sollten. Während einige Autoren wenigstens eine Variable integriert haben, die Hinweise auf die direkten oder indirekten Kosten der Ausbildung geben konnten, wurden die Ertragserwartungen in den Untersuchungen weitgehend ignoriert. Dies erstaunt insbesondere deshalb, weil die erwarteten Ertragssteigerungen in der Humankapitaltheorie das Hauptargument für private Investitionen in Ausbildung darstellen.

Für die Kostenfaktoren scheinen die Ergebnisse relativ einheitliche Zusammenhänge zu beschreiben. Im Vergleich zu den direkten Kosten des Schulbesuchs, die in den vorliegenden Untersuchungen kaum eine Rolle spielen, stellen die entstehenden Opportunitätskosten einen wichtigen Entscheidungsfaktor in der "Bildungsnachfrage“ dar. Dieses Ergebnis erstaunt nicht, da in den meisten Entwicklungsländern auch eine staatliche Basisausbildung kostenlos oder zu geringen Gebühren angeboten wird. Da in Entwicklungsländern oft schon die Kinder zum Einkommenserwerb beitragen oder traditionelle Rollen im Haushalt übernehmen, überrascht auch die Wirkung der Opportunitätskosten nicht. Sowohl für die direkten Kosten als auch für die Opportunitätskosten kann angenommen werden, dass sie für arme Haushalte mehr Relevanz haben als für wohlhabendere Haushalte.

Die größere Relevanz der Kosten in armen Familien wird durch die Beschränkung des Kreditmarktes erklärt. Wenn sie Versorgungsengpässe durch besonders teure informelle Kredite ausgleichen müssen, sind die Opportunitätskosten des Schulbesuchs besonders hoch.

Der Zusammenhang zwischen den erwarteten Nutzen der Ausbildung und der „Bildungsnachfrage“ bleibt dagegen weitgehend unerforscht. Die schwierige Bewertung dieser erst zukünftig anfallenden Ergebnisse von Ausbildung in den Familien selber und die problematische Erhebung dieser Daten bilden den Grund für die schwache Datenlage. 
Die wenigen Untersuchungen, die es zu den erwarteten Erträgen von Ausbildung gibt, setzten diese nicht in Beziehung zur „Bildungsnachfrage“. Es wurde ermittelt, dass Studenten ein relativ realistisches Bild von der Arbeitsmarktsituation haben, obwohl ihre Erwartungen weit streuen und systematisch mit persönlichen Merkmalen variieren. Die Befragungen wurden allerdings nur in höheren Bildungsstufen in den USA durchgeführt. Die Ergebnisse geben daher nur begrenzt Aufschluss über die Ertragserwartungen von Familien für die Basisausbildung ihrer Kinder in Entwicklungsländern.

Wenn die Ertragserwartungen tatsächlich das Hauptargument für die Bildungsentscheidung darstellen (wie es die Humankapitaltheorie annimmt), können durch unterschiedliche Wahrnehmungen derselben $u$. U. große Teile der Nachfrageunterschiede zwischen Bevölkerungsgruppen erklärt werden. Die Untersuchungen zeigen erhebliche Variationen in den Erwartungen. Leider gibt es dazu kaum Untersuchungen und erst recht keine nach Einkommensgruppen differenzierten Ergebnisse. Hier besteht noch erheblicher Forschungsbedarf. 



\section{Familiäre und individuelle Merkmale als Bildungsdeter- minanten}

Im Gegensatz zu der vagen Behandlung der Nutzen- und Kostenfaktoren, beinhalten alle empirischen Untersuchungen über die „Bildungsnachfrage“ in Entwicklungsländern Variablen, die die familiäre Situation der untersuchten Haushalte und die betroffenen Kinder beschreiben sollen. Die Autoren scheinen also der Kritik an der ökonomischen Verhaltenstheorie gefolgt zu sein, und den individuellen Charakteristika (die Einfluss auf die Präferenzen haben) einen besonders hohen Erklärungswert für die Bildungsentscheidungen beizumessen. Einerseits wird angenommen, dass nicht nur die Nutzen- und Kostenfaktoren die Investitionsentscheidungen beeinflussen, sondern dass auch in den Familien selber die Ursache für unterschiedliches Nachfrageverhalten begründet sein kann. Andererseits sind die direkten Einflussfaktoren oft nicht beobachtbar oder in den Datensätzen nicht erfragt worden. In diesem Fall können die Familienvariablen Hinweise auf die Wirksamkeit anderer Faktoren geben.

\subsection{Haushaltscharakteristika als Bildungsdeterminanten}

Um die Haushalte zu beschreiben, werden vor allem die familiären Einkommensverhältnisse und die Bildungsniveaus der Eltern herangezogen. Dieses Interesse ist erklärbar. Eine umfassende Basisausbildung aller Bevölkerungsgruppen wird als adäquates Mittel zur Armutsbekämpfung betrachtet. Dabei wird davon ausgegangen, dass besser ausgebildete Individuen durch die Erhöhung ihrer Arbeitsproduktivität höhere Einkommen erzielen und damit aus der Armut entfliehen können. ${ }^{191}$ Dazu ist es aber notwendig, dass vor allem die armen Bevölkerungsgruppen das Bildungsangebot nutzen. Auch die weniger ausgebildeten Eltern müssen in der Schulbildung eine Entwicklungschance für ihre Kinder erkennen. Fragen arme oder weniger ausgebildete Eltern weniger Bildung für ihre Kinder nach, ist die Gefahr groß, dass die Armut von Generation zu Generation weiter gegeben wird. Daher ist es besonders wichtig, die Nachfragedeterminanten aufzudecken, die zu dieser Entwicklung führen könnten.

Fast jede Untersuchung enthält außerdem eine Variable für die Familienstruktur. Dabei wird vor allem angenommen, dass die Anzahl der Geschwister Aus-

191 Vgl. Sautter/Hesselbarth (1998), S. 330-336; Almeida/Camargo (1994), S. 2; Barham/Boadway/ Marchand/Pestieau (1994), S. 1258 und Dabla-Norris/Matovu/Wade (2002), S. 16. Alle Autoren weisen auf den Konsens unter Ökonomen und Politikern hin, dass über eine umfassende Ausbildung sowohl Wachstums- als auch Verteilungsziele erreicht werden können. 
wirkungen auf die Ausbildung des einzelnen Kindes hat. Auch hier soll wieder untersucht werden, ob das Einkommen eine Begrenzung darstellt.

\subsubsection{Einkommen}

Aufgrund der schwierigen Datenlage sind die Einkommen einer Familie oft nicht eindeutig zu ermitteln oder brauchbar zu instrumentalisieren. ${ }^{192}$ Einige Autoren greifen daher auf die Konsumausgaben zurück oder ziehen Besitztümer als Wohlstandsindikatoren heran. Die Interpretation der Ergebnisse ist schwierig, da nicht genau getrennt werden kann, über welchen Kanal das Einkommensniveau die Ausbildung beeinflusst. Einerseits wurde gezeigt, dass die Mittelknappheit an sich ein Investitionshindernis darstellt193, andererseits können Familien in unterschiedlichen Einkommensschichten auch einfach unterschiedliche subjektive Wahrnehmungen und Präferenzen aufweisen. Inwieweit die Untersuchungsergebnisse die Zusammenhänge aufklären können, wird im folgenden untersucht.

Zunächst werden die Untersuchungen dargestellt, die tatsächlich eine Variable für das Einkommen integriert haben. Bei der Durchsicht der auf der folgenden Seite dargestellten Tabelle fällt auf, dass die beiden Studien über die USA einen deutlichen Effekt des Einkommens ermittelten ${ }^{194}$, der für Entwicklungsländern nicht bestätigt werden konnte. Dies überrascht, da in Entwicklungsländern wegen der Knappheit der familiären Ressourcen mit einem höheren Einfluss des Einkommens gerechnet wurde. Allerdings können die Ergebnisse für die USA damit erklärt werden, dass dort vor allem die Nachfrage nach höherer Bildung untersucht wurde, während es in den Studien zu Entwicklungsländern um Basisausbildung geht.

Dennoch zeigt sich in den meisten Studien auch für Entwicklungsländer ein positiver Effekt des Einkommens, der allerdings oft schwach und in einigen Untersuchungen nicht statistisch signifikant ist.

\footnotetext{
192 Vgl. Psacharopoulos (1989), S. 689.

193 Vgl. die Ausführungen zu den Opportunitätskosten in Abschnitt 5.2.1.2 und zu den Kapitalmarktrestriktionen in Abschnitt 5.2.3.

194 Vgl. Taubmann (1989), S. 60. Als Ergebnis seiner Untersuchung stellte McMahon fest, dass Unterschiede in den finanziellen Bedingungen einer Familie für die Erklärung von Bildungsunterschieden eine größere Rolle spielen als Unterschiede in der Leistungsfähigkeit. Die Signifikanzniveaus und die Betakoeffizienten für das Familieneinkommen, Studentendarlehen und Stipendien sind alle höher als die für Ability, elterliche Bildung oder Unsicherheit über zukünftige Einkommen. Vgl. McMahon (1984), S. 88-89.
} 
Tabelle 7.1: Familieneinkommen als Bildungsdeterminante

\begin{tabular}{|c|c|c|c|c|c|}
\hline Autor & Land & Zielgruppe & abhängige Variable & erkl. Variable & Ergebnis \\
\hline Birdsall, 1985 & Brasilien & 8-15 Jahre & abgeschlossene Schuljahre & Log Einkommen d. Vaters & positiv, Stadt > Land \\
\hline $\begin{array}{l}\text { Burney/Irfan, } \\
1995\end{array}$ & $\begin{array}{l}\text { Pakistan, } \\
\text { ländlich }\end{array}$ & 10-25 Jahre & Bildungsteilnahme, j/n & $\begin{array}{l}\text { monatliches Einkommen des } \\
\text { Haushalts }\end{array}$ & $\begin{array}{l}\text { positiv, } \\
\text { nur z. T. signifikant }\end{array}$ \\
\hline \multirow[t]{2}{*}{ Jacoby, 1994} & \multirow[t]{2}{*}{ Peru } & \multirow[t]{2}{*}{ 7-12 Jahre } & Wahrscheinlichkeit des Rückfalls & Eink. aus selbst. Tätigkeit & negativ \\
\hline & & & Wahrscheinlichkeit des Rückfalls & andere Einkommen & negativ \\
\hline \multirow[t]{2}{*}{ Lavy, 1996} & \multirow{2}{*}{$\begin{array}{l}\text { Ghana, } \\
\text { ländlich }\end{array}$} & \multirow[t]{2}{*}{ 5-12 Jahre } & jemals teilgenommen & Log PKE & nicht signifikant \\
\hline & & & Grade Attainment & Log PKE & nicht signifikant \\
\hline $\begin{array}{l}\text { Leibovich/ } \\
\text { Magnac, } 1996\end{array}$ & $\begin{array}{l}\text { Kolumbien, } \\
\text { ländlich }\end{array}$ & 5-17 Jahre & Bildungsteilnahme, j/n & PKE & nicht signifikant \\
\hline \multirow{2}{*}{$\begin{array}{l}\text { Levison/Moe, } \\
1998\end{array}$} & \multirow{2}{*}{$\begin{array}{l}\text { Peru, } \\
\text { ländlich }\end{array}$} & \multirow{2}{*}{$\begin{array}{l}\text { 10-19, nur } \\
\text { Mädchen }\end{array}$} & Bildungsteilnahme, j/n & Fam. Eink. & negativ \\
\hline & & & wöchentliche Zeit in der Schule & Fam. Eink. & nicht signifikant \\
\hline \multirow{2}{*}{$\begin{array}{l}\text { Psacharopoulos/ } \\
\text { Arriagada, } 1989 \\
\end{array}$} & \multirow[t]{2}{*}{ Brasilien } & \multirow[t]{2}{*}{ 7-14 Jahre } & Bildungsteilnahme & monatl. Eink. & positiv aber niedrig \\
\hline & & & Grade Attainment & monatl. Eink. & positiv aber niedrig \\
\hline McMahon, 1984 & USA & College age & gepl. Investition (Jahre x Kosten) & disponibles Einkommen & $\begin{array}{l}\text { hoch signifikant, Jun- } \\
\text { gen > Mädchen }\end{array}$ \\
\hline Taubman, 1989 & \multicolumn{2}{|c|}{ Zusammenfassung USA } & diverse & Einkommen, diverse & $\begin{array}{l}\text { positiv mit Elastizitä- } \\
\text { ten zw. } 3 \text { u. } 80 \%\end{array}$ \\
\hline
\end{tabular}


BIRDSALL differenzierte ihre Ergebnisse nach Regionen und stellt fest, dass der Einkommenseffekt in Städten größer ist als auf dem Land. Für die städtische Stichprobe steigt der Effekt außerdem signifikant mit dem Alter an. ${ }^{195}$ Hier wird die oben genannte Vermutung bestätigt, dass das Einkommen bei höherer Bildung an Einfluss gewinnt. Dies kann zum einen daran liegen, dass höhere Bildung höhere Kosten verursacht und deshalb die Einkommensrestriktionen an Bedeutung gewinnen. Zum anderen kann dies aber auch bedeuten, dass reichere Familien der höheren Bildung mehr Wert beimessen und arme Familien diese Bildungsstufe nicht als eine für sie relevante Alternative betrachten.

BIRDSALL integrierte außerdem einige Angebotsfaktoren (Verfügbarkeit und Qualität der Schulen) in die Regressionen und konnte eine deutliche Abnahme der Einkommenskoeffizienten zeigen; der Effekt blieb aber signifikant. Sie integriert außerdem Variablen, die die Wechselwirkungen zwischen Haushaltscharakteristika und Angebotsbedingungen darstellen. Für die städtische Stichprobe stellt sie fest, dass Unterschiede in der Verfügbarkeit und der Qualität der Schulen mit steigendem Einkommen und höherem Bildungsniveau der Mutter an Einfluss verlieren. ${ }^{196}$ Das heißt, dass Kinder armer Familien relativ mehr von Verbesserungen im Bildungsangebot profitieren, während wohlhabendere Familien mit besser ausgebildeten Eltern Mängel im Angebot besser kompensieren können.

BURNEY/IRFAN ermittelten einen schwachen positiven Einfluss des monatlichen Haushaltseinkommens. Die Koeffizienten sind allerdings nur für Männer und in Nicht-Farm Haushalten sogar nur für Männer zwischen 17 und 20 Jahren signifikant. ${ }^{197}$

JACOBY zeigte, dass das Einkommen (unabhängig ob selbständig oder abhängig erwirtschaftet) die Wiederholungswahrscheinlichkeit deutlich senkt. Um zu beweisen, dass der Einkommenseffekt nicht ausschließlich aus nicht zu beobachtbaren Motivationen (Präferenzen) resultiert, teilt JACOBY die Stichprobe nach dem Zugang zum Kapitalmarkt ein. Dabei ermittelt er, dass wohlhabendere Haushalte signifikant weniger wahrscheinlich Kreditrationierungen unterliegen. Wenn die Stichprobe nach der Wahrscheinlichkeit einer Kreditrationierung zu unterliegen aufgeteilt wird, zeigt sich, dass höhere Einkommen den Bildungserfolg nicht signifikant steigern, wenn der Haushalt Zugang zum Kapitalmarkt hat. In kreditbeschränkten Haushalten hingegen hat das Einkommensni-

195 Vgl. Birdsall (1985), S. 79-83.

196 Die Unterschiede in den Nachfrageelastizitäten (bezogen auf Veränderungen in den Angebotsvariablen) zwischen Einkommensgruppen sind in Städten stärker ausgeprägt als auf dem Land. Vgl. Birdsall (1985), S. 84.

197 Vgl. Burney/Irfan (1995), S. 29. 
veau einen signifikanten Einfluss. Der Grund scheint also nicht ausschließlich in unterschiedlichen Präferenzen in den Einkommensgruppen zu liegen sondern tatsächlich in finanziellen Restriktionen. Auch wenn die Konsumausgaben als Wohlstandsmaß herangezogen werden, ändert sich das Ergebnis nicht. ${ }^{198}$ LEIBOVICH/MAGNAC werten eine Haushaltsbefragung für das ländliche Kolumbien aus. Die Daten zeigen einen Zusammenhang zwischen der Wahrscheinlichkeit, nach dem Bachillerato weiter die Schule zu besuchen und dem Einkommen der Familien. Während für Familien, deren Einkommen über dem Durchschnitt liegen, die Wahrscheinlichkeit für einen weiteren Schulbesuch der Kinder noch über 75\% liegt, sinkt diese Wahrscheinlichkeit für Kinder aus Familien mit mittleren Einkommen auf ca. 20\% und für Familien mit niedrigeren Einkommen sogar auf unter $10 \%$. In der ökonomischen Überprüfung ihres Modells können sie diesen deutlichen Zusammenhang zwischen dem Einkommen und der Bildungsbeteiligung allerdings nicht bestätigen. Dennoch bezweifeln die Autoren den Effekt der Einkommen auf die Ausbildung der Kinder nicht. ${ }^{199}$ Vielmehr verweisen sie auf die Probleme in der Datenlage und der Instrumentalisierung der Variablen.

Auch PSACHAROPOULOS/ARRIAGADA führen als mögliche Ursache für den schwachen Effekt die Probleme bei der Bestimmung des Familieneinkommens aus Haushaltsdaten an. 200

LAVY (1996) konnte für das ländliche Ghana keinen besonderen Einfluss der Pro-Kopf-Einkommen auf das Bildungsverhalten ermitteln. ${ }^{201}$ Und LEVISON/MOE ermittelten sogar einen negativen Einfluss des Einkommens auf die Bildungsbeteiligung für junge Mädchen im ländlichen Peru. Allerdings ist der Effekt sehr klein. 202

Die folgende Tabelle zeigt, dass mit den Konsumausgaben der Familie als Wohlstandsvariable der erwartete Zusammenhang etwas deutlicher nachzuweisen ist. Dies könnte an der besseren Messbarkeit der Variable liegen.

\footnotetext{
198 Vgl. Jacoby (1994), S. 158-159.

199 Vgl. Leibovich/Magnac (1996), S. 138-141.

200 Vgl. Psacharopoulos/Arriagada (1989), S. 698 und700.

201 Vgl. Lavy (1996), S. 300-304.

202 Vgl. Levison/Moe (1998), S. 349-350.
} 
Tabelle 7.2: Konsumausgaben als Bildungsdeterminante

\begin{tabular}{l|l|l|l|l|l} 
Autor & Land & Zielgruppe & abhängige Variable & erklärende Variable & Ergebnis \\
\hline $\begin{array}{l}\text { Gertler/ } \\
\text { Glewwe, 1992 }\end{array}$ & $\begin{array}{l}\text { Peru, } \\
\text { ländlich }\end{array}$ & $\begin{array}{l}\text { 10-18 Jahre, } \\
\text { Sekundarschule }\end{array}$ & Teilnahme, j/n & $\begin{array}{l}\text { Pro-Kopf Konsum, Pro- } \\
\text { Kopf Konsum }{ }^{2}\end{array}$ & positiv, nicht linear \\
\hline $\begin{array}{l}\text { Glewe/Grosh/Jaco- } \\
\text { by/Lockheed, 1995 }\end{array}$ & Jamaika & Primarschule & Auswahl der All-Age School & Pro-Kopf Ausgaben & nicht signifikant \\
\cline { 5 - 7 } Handa, 1996 & Jamaika & $\begin{array}{l}\text { Sekundarschule, } \\
\text { 13-19 Jahre }\end{array}$ & Testergebnis, Mathematik & Pro-Kopf Ausgaben & positiv \\
\cline { 5 - 7 } & akademischer Schultyp, j/n & Log Pro-Kopf Ausgaben & positiv, nur für Mädchen \\
\hline Jacoby, 1994 & Peru & 7-12 Jahre & $\begin{array}{l}\text { Wahrscheinlichkeit des } \\
\text { Rückfalls }\end{array}$ & Konsumausgaben & negativ \\
\hline De Vreyer, 1993 & $\begin{array}{l}\text { Elfen- } \\
\text { beinküste }\end{array}$ & 5-25 Jahre & Anzahl Schuljahre & Pro-Kopf Ausgaben & $\begin{array}{l}\text { positiv, in Städten und } \\
\text { für Mädchen }\end{array}$ \\
\hline Skyt Nielsen, 1998 & Sambia & $\begin{array}{l}\text { 7-18 Jahre, } \\
\text { Primarschule }\end{array}$ & Z. Z. Teilnahme, j/n & $\begin{array}{l}\text { Armutsmaß, 3 Kategorien } \\
\text { extremer. Armut }\end{array}$ & $\begin{array}{l}\text { negativ, nur für das } \\
\text { ärmste Drittel signifikant }\end{array}$ \\
\hline
\end{tabular}


GeRTLER/GLEWWE berücksichtigen den Pro-Kopf-Konsum der Haushalte in ihrer Nachfragefunktion für Sekundarbildung im ländlichen Peru. Die Koeffizienten für den Konsum und den quadrierten Konsum besagen, dass die Nachfrage nach Schulbildung mit dem Ausgabenniveau signifikant steigt aber nicht linear ist. Außerdem ermitteln die Autoren insgesamt niedrige Preiselastizitäten für die „Bildungsnachfrage“, die aber mit sinkendem Einkommensniveau steigen. ${ }^{203}$ Die „Bildungsnachfrage“ reagiert also nur schwach auf eine Gebührenanhebung. Arme Haushalte wären aber überproportional am Bildungsrückgang beteiligt.

Glewwe/GRosh/JAcoby/LockHEED untersuchten den Einfluss der Pro-KopfAusgaben auf die Auswahl des Primarschultyps in Jamaika. Obwohl beide öffentlichen Schultypen das selbe Curriculum und die gleichen Schulbücher benutzen, ist die All-Age-School vornehmlich im abgelegenen ländlichen Raum anzutreffen und oft weniger gut ausgestattet. Von Schülern der Primarschule wird erwartet, dass sie später die akademische High School besuchen, während Schüler der All-Age-Schools häufiger in den Arbeitsmarkt entlassen werden. ${ }^{204}$ Die Autoren konnten keinen Einfluss des Ausgabenniveaus auf die Schulwahl feststellen. 205

Auch HANDA untersuchte das Bildungsverhalten von Jamaikanern. Allerdings bezieht sich ihre Studie auf die Determinanten der Sekundarbildung und stellte einen positiven Einfluss der Konsumausgaben fest. ${ }^{206}$ Für die grundsätzliche Teilnahmeentscheidung an einer sekundären Ausbildung hat das Ausgabenniveau allerdings nur für Mädchen einen großen Einfluss. Dieser Einfluss auf die Mädchen ist allerdings etwas überbewertet. Bei Integration einer Variablen für das Vorhandensein jüngerer Geschwister (signifikant negativ für Mädchen) sinkt der Einfluss des Einkommens etwas, bleibt aber signifikant. Die Anzahl jüngerer Geschwister scheint also negativ mit dem Einkommen korreliert zu sein. Für Jungen wirkt dieser Mechanismus umgekehrt (jüngere Geschwister erhöhen die

203 Vgl. Gertler/Glewwe (1992), S. 183 und 185. Wie oben bereits dargestellt, wird der Preis anhand von Gebühren gemessen. Für Mädchen werden bei jedem Einkommensniveau höhere Elastizitäten ermittelt, womit sie von einer Anhebung der Gebühren besonders betroffen wären. Vgl. Punkt 5.2.1 zu den direkten Kosten.

204 Vgl. Glewwe/Gertler/Jacoby/Lockheed (1995), S. 236.

205 Vgl. Glewwe/Gertler/Jacoby/Lockheed (1995), S. 243-244. Zusätzlich ermittelten die Autoren den Einfluss auf die Testergebnisse in Mathematik und Lesen. Hier erhielten sie einen starken positiven und signifikanten Einfluss des Ausgabenniveaus auf die Leistungen in Mathematik. Eine Erhöhung der Ausgaben pro Kopf um eine Standardabweichung induziert eine Testverbesserung um 43 Punkte - was fast dem Effekt eines zusätzlichen Schuljahres entspricht. Auf die Lesefähigkeit konnte kein Einfluss des Ausgabenniveaus festgestellt werden. Vgl. Glewwe/Gertler/Jacoby/ Lockheed (1995), S. 248.

206 Vgl. Handa (1996), S. 561-562. Die Ausgaben werden herangezogen, weil keine Angaben zu den Einkommen vorhanden waren. Die potentielle Endogenität wurde korrigiert. Vgl. S. 559. 
Einschulungswahrscheinlichkeit), was zu einer Unterschätzung des Einkommenseffektes führt und die Diskrepanz zwischen den Geschlechtern mindert.207 Bei der Entscheidung, eine High School (die akademische Variante der Sekundaria und Voraussetzung für weitere Bildung) zu besuchen, ist der Effekt des Einkommens noch stärker und nun für beide Geschlechter signifikant. Aber auch hier reagiert die Wahrscheinlichkeit der Einschulung für Mädchen doppelt so stark auf eine Variation der Einkommen. Eine Erhöhung der Einkommen um eine Standardabweichung führt bei Mädchen zu einer 10,5\%igen Steigerung der Wahrscheinlichkeit, eine High School zu besuchen und bei Jungen nur zu einer Erhöhung um 5,5\%. Das heißt umgekehrt, dass Einkommensbeschränkungen für Mädchen bindender sind. Dieser Einfluss des Einkommens bleibt zwar signifikant, wird aber deutlich reduziert wenn kommunale Unterschiede berücksichtigt werden (5,5\%, 1,5\%). Offensichtlich ist also ein großer Teil des Einkommenseffektes durch ein unterschiedliches Angebot an kommunalen Einrichtungen erklärbar. ${ }^{208}$

Dennoch zeigt HANDA, dass das Einkommen ein wichtiger Einflussfaktor für die Sekundarbildung in Jamaika ist. Für die High School ist das Einkommen sogar die Einflussreichste Variable unter den Haushaltscharakteristika. Sie zieht daraus den Schluss, dass das dualistische Sekundarschulsystem in Jamaika die Ungleichheit perpetuiert, da es die Kinder reicherer Haushalte in den akademischen Elite High Schools plaziert, während die Kinder armer Haushalte vornehmlich die qualitativ minderwertigeren All-Age-Schulen besuchen. 209

Die beiden Studien über die jamaikanische „Bildungsnachfrage“ unterstützen also die Vermutung, dass das Einkommen erst bei höheren Bildungsniveaus an Bedeutung gewinnt.

DE VREYER ermittelte einen positiven und stark signifikanten Effekt der familiären Pro-Kopf-Ausgaben auf das Bildungsniveau der Kinder in der Elfenbeinküste. Allerdings ist auch hier der Effekt relativ niedrig. Differenziert nach der Region und dem Geschlecht der Schüler ergibt sich ein nicht signifikantes Ergebnis für ländliche Gegenden und für Jungen. In beiden Fällen hat dafür die Produktion von Festpreisprodukten größere Relevanz. ${ }^{210}$ Hier scheint der Sicherheit der Einkommen eine größere Rolle zuzukommen als der Höhe der Einkommen.

\footnotetext{
207 Vgl. Handa (1996), S. 569.

208 Vgl. Handa (1996), S. 573.

209 Vgl. Handa (1996), S. 576.

210 Vgl. De Vreyer (1993), S. 71-72.
} 
SKYT NIELSEN führte in ihre Untersuchungen ein Armutsmaß211 ein, um den Einfluss der Einkommensverhältnisse auf die Bildungsbeteiligung in Sambia zu ermitteln. Die Armen werden nach ihren Konsumausgaben nochmals in drei Gruppen eingeteilt. Die Analyse zeigt, dass der Armutseffekt für die Bildungsbeteiligung nur gering ist. Die Koeffizienten sind zwar für alle Haushalte unter der Armutslinie negativ, aber nur für das ärmste Drittel der Armen signifikant. Hier zeigen sich auch die höchsten Werte. Eine Erhöhung des Ausgabenniveaus des ärmsten Drittels der Armen über die extreme Armutslinie, würde die Bildungsbeteiligung für 9jährige um 0,2 Prozentpunkte für Mädchen und um 0,1 Prozentpunkt für Jungen steigern. Für 15jährige steigen diese Effekte auf 3,8 bzw. 2,3 Prozentpunkte. SKYT NiELSEN folgert daraus, dass die Reduzierung der Armut ein teurer Weg ist, die Bildungsbeteiligung zu erhöhen. Andererseits ist die Steigerung der Bildungsbeteiligung aber ein schöner Nebeneffekt einer armutsreduzierenden Sozialpolitik. ${ }^{212}$ Auch hier zeigt sich wieder ein stärkerer Einfluss des Einkommens auf die Ausbildung der Mädchen und bei der Entscheidung über höhere Bildungsniveaus (Alter der Kinder).

211 Sie definiert eine Armutslinie auf der Basis eines Warenkorbes für einen männlichen Erwachsenen. Obwohl die definierte Armutslinie ein Maß für extreme Armut darstellt, fallen 67\% der Stichprobe darunter.

212 Vgl. Skyt Nielsen (1998), S. 19. 
Eine weitere Variable, die den Wohlstand einer Familie widerspiegeln soll, bestimmt den Besitz von Wertgegenständen. Auch hier ergeben sich die erwarteten Zusammenhänge.

\section{Tabelle 7.3: Besitz von Wertgegenständen als Bildungsdeterminante}

\begin{tabular}{l|l|l|l|l|l} 
Autor & Land & Zielgruppe & abh. Variable & erkl. Variable & Ergebnis \\
\hline $\begin{array}{l}\text { Bedi/Mar- } \\
\text { shall, 1999 }\end{array}$ & $\begin{array}{l}\text { Honduras, } \\
\text { ländlich }\end{array}$ & $\begin{array}{l}\text { Primaria, } \\
\text { 1.-3. Klasse }\end{array}$ & $\begin{array}{l}\text { Anwesenheit, Ta- } \\
\text { ge im Schuljahr }\end{array}$ & $\begin{array}{l}\text { Besitz von Wert- } \\
\text { gegenständen }\end{array}$ & $\begin{array}{l}\text { positiv für die } \\
\text { 1. u. 2. Klasse }\end{array}$ \\
\hline $\begin{array}{l}\text { Jacoby, } \\
1994\end{array}$ & Peru & 7-12 Jahre & $\begin{array}{l}\text { Wahrscheinlichkeit } \\
\text { des Rückfalls }\end{array}$ & $\begin{array}{l}\text { langlebige } \\
\text { Gebrauchsgüter }\end{array}$ & negativ \\
$\begin{array}{l}\text { Skyt Niel- } \\
\text { sen, } 1998\end{array}$ & Sambia & $\begin{array}{l}\text { 7-18 Jahre, } \\
\text { Primaria }\end{array}$ & z. Z. Teilnahme, j/n & $\begin{array}{l}\text { langlebige } \\
\text { Gebrauchsgüter }\end{array}$ & positiv \\
\hline
\end{tabular}

BEDI/MARSHALL integrierten in ihre Schätzungen der Bildungsbeteiligung im ländlichen Honduras eine Dummy-Variable, um den Familienwohlstand auszudrücken. Die Variable nimmt den Wert eins an, wenn der Haushalt ein Fernsehund Radiogerät besitzt. Der Effekt dieser Wohlstandsvariable ist groß und statistisch signifikant für die erste und zweite Klasse. Kinder wohlhabender Haushalte sind zwischen fünf und neun Tagen häufiger anwesend während eines Schuljahres. ${ }^{213} \mathrm{Da}$ auch in Honduras für die staatliche Primarschule kaum Gebühren verlangt werden, führen die Autoren dieses Ergebnis auf die Wirkung der Opportunitätskosten zurück. ${ }^{214}$ Dass der Wohlstand hier schon für die Grundschule eine bedeutende Rolle spielt, aber in der dritten Klasse keine statistische Signifikanz mehr besitzt, widerspricht allerdings dem steigenden Einfluss von Einkommen mit dem Bildungsniveau. Allerdings ist die Grundschule wohl eher als Einheit zu betrachten. Wenn die Einschulungsentscheidung gefallen ist, verliert das Einkommen an Relevanz bis die nächste grundsätzliche Entscheidung ansteht.

Wie schon die bei höherem Einkommen und bei höherem Ausgabenniveau weisen bei JACOBY auch die Kinder aus Familien mit mehr Besitz von langlebigen Gebrauchsgütern eine signifikant niedrigere Wahrscheinlichkeit auf, in der Schule zurück zu fallen. Diesen positiven Zusammenhang bestätigt auch die Untersuchung von SKYT NIELSEN.

Wie die obigen Untersuchungen zeigen, ermittelten die Autoren für die meisten Länder einen positiven Zusammenhang zwischen dem Einkommensniveau einer Familie und der „Bildungsnachfrage“ für ihre Kinder. Allerdings zeigen einige Berechnungen nur einen sehr niedrigen Koeffizienten für die Einkommensvariable. Diese Ergebnisse bleiben auch stabil, wenn unterschiedliche Indikato-

213 Vgl. Bedi/Marshall (1999), S. 669.

214 Wohlhabende Familien sind weniger empfindlich gegenüber Kapitalmarktrestriktionen. Sie können zum Beispiel Geld leihen, um Arbeitskräfte zu bezahlen und sind damit weniger angewiesen auf die Mitarbeit ihrer Kinder. Vgl. zu den Opportunitätskosten die Ausführungen in Kapitel 5.3.2. 
ren für das Einkommensverhältnis der Haushalte herangezogen werden. Wenn andere als die erwarteten Ergebnisse berechnet wurden, äußerten die Autoren Zweifel an der Qualität der Einkommensdaten.

Die meisten Untersuchungen beziehen sich auf den ländlichen Raum in Entwicklungsländern. Die einzigen Untersuchungen, die sich auf das gesamte Land beziehen und nach Regionen differenzieren ${ }^{215}$, weisen für die Städte eine höhere Abhängigkeit der „Bildungsnachfrage“ vom Einkommen aus. Auch Nachfrageelastizitäten zeigen einen höheren Wert für arme Haushalte und stärkere Differenzen in städtischen Regionen.

Außerdem zeigen die Untersuchungen, dass in der Einkommensvariablen auch andere Einflüsse versteckt sein können. Die zusätzliche Berücksichtigung von Faktoren zum Bildungsangebot, der Anwesenheit kleinerer Geschwister oder kommunalen Unterschieden senkten alle den Koeffizienten der Einkommensvariablen, ließen das Ergebnis aber statistisch signifikant.

\subsubsection{Bildungsniveau der Eltern}

Das Bildungsniveau der Eltern gilt als Maß für die Präferenzen der Eltern gegenüber der Schule, für die Weitsichtigkeit der Planungen und als Einflussfaktor auf die Fähigkeit der Kinder, von der Ausbildung zu profitieren. Eine bessere Ausbildung der Eltern erhöht die elterlichen Fähigkeiten, die Ausbildung der Kinder zu lenken und zu unterstützen. Wenn verwendbare Angaben zum Einkommensniveau der Eltern fehlen, kann die Ausbildung der Eltern auch einen Teil der Einkommenseffekte reflektieren - soweit die Einkommen und die Ausbildung der Eltern korreliert sind. Wenn besser ausgebildete Eltern ihren Kindern mehr Ausbildung zukommen lassen und die Ausbildung mit dem Einkommen korreliert ist, wird durch diese Zusammenhänge die Ungleichverteilung der Einkommen perpetuiert. Da die Verteilung der Einkommen ein zentrales Argument der Armutsbekämpfung darstellt, wird das elterliche Bildungsniveau in jeder Untersuchung der Bildungsdeterminanten berücksichtigt. Die folgende Tabelle zeigt ein sehr einheitliches Bild.

215 Dies sind nur zwei: DeVreyer für die Elfenbeinküste und Psacharopoulos/Arriagada für Brasilien. 
Tabelle 7.4: Bildungsniveau der Eltern als Bildungsdeterminante

\begin{tabular}{|c|c|c|c|c|c|}
\hline Autor & Land & Zielgr. & abhängige Variable & erklärende Variable & Ergebnis \\
\hline Birdsall, 1985 & Brasilien & $8-15 \mathrm{~J}$. & abgeschl. Schuljahre & Schuljahre Vater, Mutter & positiv, $M>V$ \\
\hline \multirow[t]{2}{*}{ Burney/Irfan, 1995} & \multirow{2}{*}{$\begin{array}{l}\text { Pakistan, } \\
\text { ländlich }\end{array}$} & \multirow[t]{2}{*}{$10-25 \mathrm{~J}}$. & \multirow{2}{*}{ Bildungsbeteiligung, j/n } & Bildung Vater, 3 Stufen & nur ,Higher' positiv \\
\hline & & & & Bildung Mutter, 3 Stufen & nur ,Higher' pos. f. Nichtfarmer \\
\hline \multirow{2}{*}{$\begin{array}{l}\text { Gertler/ Glewwe, } \\
1992\end{array}$} & \multirow{2}{*}{$\begin{array}{l}\text { Peru, } \\
\text { ländlich }\end{array}$} & \multirow[t]{2}{*}{$10-18 \mathrm{~J}}$. & Teilnahme, j/n & Schuljahre Vater & nicht signifikant \\
\hline & & & Teilnahme, $\mathrm{j} / \mathrm{n}$ & Schuljahre Mutter & nicht signifikant \\
\hline \multirow{3}{*}{$\begin{array}{l}\text { Glewwe/Grosh/ } \\
\text { Jacoby/Lockheed, } \\
1995\end{array}$} & \multirow[t]{3}{*}{ Jamaika } & \multirow{3}{*}{$\begin{array}{l}\text { Primar- } \\
\text { schule }\end{array}$} & All-Age School, j/n & Schuljahre Vater & negativ \\
\hline & & & All-Age School, j/n & Schuljahre Mutter & nicht signifikant \\
\hline & & & Tests in Mathe, Lesen & Schuljahre Vater, Mutter & nicht signifikant \\
\hline \multirow[t]{4}{*}{ Handa, 1996} & \multirow[t]{4}{*}{ Jamaika } & \multirow{4}{*}{$\begin{array}{l}\text { Sekundar- } \\
\text { schule } \\
13-19 \mathrm{~J} .\end{array}$} & Teilnahme, j/n & Bildungsniveau Mutter & positiv, nur für Mädchen \\
\hline & & & Teilnahme, $\mathrm{j} / \mathrm{n}$ & Bildungsniveau Vater & positiv, nur für Jungen \\
\hline & & & akademischer Schultyp, j/n & Bildungsniveau Mutter & nicht signifikant \\
\hline & & & akademischer Schultyp, j/n & Bildungsniveau Vater & positiv \\
\hline Jacoby, 1994 & Peru & $7-12 \mathrm{~J}$. & Wkt. des Rückfalls & Schuljahre Mutter, Vater & negativ, $M>V$ \\
\hline \multirow[t]{4}{*}{ Lavy, 1996} & \multirow{4}{*}{$\begin{array}{l}\text { Ghana, } \\
\text { ländlich }\end{array}$} & \multirow[t]{4}{*}{$5-12 \mathrm{~J}}$. & jemals teilgenommen & Primaria Vater, Mutter & positiv, $M>V$ \\
\hline & & & jemals teilgenommen & Post-Primaria Vater, Mutter & nicht signifikant \\
\hline & & & Grade Attainment & Primaria Vater, Mutter & positiv, $M>V$ \\
\hline & & & Grade Attainment & Post-Primaria Vater, Mutter & nicht signifikant \\
\hline \multirow{4}{*}{$\begin{array}{l}\text { Leibovich/ } \\
\text { Magnac,1996 }\end{array}$} & \multirow{4}{*}{$\begin{array}{l}\text { Kolumbien } \\
\text { ländlich }\end{array}$} & \multirow[t]{4}{*}{$5-17 \mathrm{~J}}$. & Bildungsbeteiligung & Bildungsniveau Vater & positiv, größer für Jungen \\
\hline & & & Bildungsbeteiligung & Bildungsniveau Mutter & positiv, größer für Mädchen \\
\hline & & & Bildungsbeteiligung & Mutter ohne Bildung, j/n & positiv \\
\hline & & & Bildungsbeteiligung & Vater ohne Bildung, j/n & positiv \\
\hline \multirow{4}{*}{$\begin{array}{l}\text { Levison/Moe, } \\
1998\end{array}$} & \multirow{4}{*}{$\begin{array}{l}\text { Peru, } \\
\text { ländlich }\end{array}$} & \multirow{4}{*}{$\begin{array}{l}\text { 10-19 J., } \\
\text { nur } \\
\text { Mädchen }\end{array}$} & wöchtl. Arbeitszeit im HH & Mutter ohne Bildung & nicht signifikant \\
\hline & & & wöchtl. Arbeitszeit im HH & Bildung Mutter, diverse Stufen & negativ \\
\hline & & & wöchtl. Zeit in der Schule & Mutter ohne Bildung & positiv \\
\hline & & & wöchtl. Zeit in der Schule & Bildung Mutter, div. Stufen & uneinheitliche Ergebnisse \\
\hline \multirow{2}{*}{$\begin{array}{l}\text { Psacharopoulos/ } \\
\text { Arriagada, } 1989\end{array}$} & \multirow[t]{2}{*}{ Brasilien } & \multirow[t]{2}{*}{$7-14 \mathrm{~J}}$. & Bildungsteilnahme, j/n & Schuljahre Vater, Mutter & positiv, $M>V$ \\
\hline & & & Bildungserfolg, Jahre & Schuljahre Vater, Mutter & positiv, $\mathrm{M}>\mathrm{V}$ \\
\hline Skyt Nielsen, 1998 & Sambia & 7-18 J. & z. Z. Teilnahme, j/n & Bildung $\mathrm{HH}$-Vorstand & positiv \\
\hline De Vreyer, 1993 & Elfenbeinküste & $5-25 \mathrm{~J}$. & Anzahl Schuljahre & Bildung $\mathrm{HH}$-Vorstand & positiv, Land $>$ Stadt, J>M \\
\hline \multirow[t]{2}{*}{ McMahon, 1984} & \multirow[t]{2}{*}{ USA } & \multirow{2}{*}{$\begin{array}{l}\text { College- } \\
\text { age }\end{array}$} & \multirow{2}{*}{$\begin{array}{l}\text { geplante Investition } \\
\text { (Jahre x Kosten) }\end{array}$} & Bildung Vater & nicht signifikant \\
\hline & & & & Bildung Mutter & nur für Jungen positiv \\
\hline
\end{tabular}


Dem Bildungsniveau der Eltern kann fast immer ein positiver Einfluss auf die Ausbildung der Kinder nachgewiesen werden. In keiner der Studien wird dem elterlichen Bildungsniveau ein negativer Effekt auf die Ausbildung der Kinder nachgewiesen, und nur GERTLER/GLEWWE konnten der elterlichen Bildung überhaupt keinen Einfluss nachweisen. ${ }^{216}$ Dennoch ist auch hier die Interpretation der Ergebnisse schwierig, da der Wirkungszusammenhang nicht eindeutig ermittelt werden kann.

BIRDSALL, JACOBY, LAVY und PSACHAROPOULOS/ARRIAGADA ermittelten alle in ihren Untersuchungen einen positiven Zusammenhang zwischen dem Bildungsniveau beider Elternteile und der Ausbildung der Kinder und stellten fest, dass das der Einfluss der mütterlichen Bildung jeweils größer ist.217 PSACHAROPOULOS/ARRIAGADA ermittelten außerdem noch niedrigere Wahrscheinlichkeiten für Kinder besser ausgebildeter Eltern einer bezahlten Tätigkeit nachzugehen und die Schule abzubrechen. Auch hier ist der Einfluss der Mutter größer. ${ }^{218}$

Außerdem integriert BIRDSALL eine Variable, die die Wechselwirkung zwischen der Ausbildung der Mutter und der Ausbildung der Lehrer (Qualität der Ausbildung) ausdrückt. Obwohl beide Variablen einzeln einen starken positiven Einfluss auf die Kinder haben, ist die Interaktionsvariable für städtische Schüler negativ. Das heißt, der positive Einfluss der Ausbildung der Mutter wird weniger wichtig je besser die städtischen Lehrer ausgebildet sind.219 Umgekehrt heißt das, dass Kinder weniger ausgebildeter Eltern relativ mehr von Verbesserungen in der Bildungsqualität profitieren. Dieser Zusammenhang lässt vermuten, dass zumindest ein Teil der Wirkung auf die bessere Unterstützung durch besser ausgebildete Mütter zurückgeht.

GLEWWE/GRosh/JACoby/LockHEED konnten für das Bildungsniveau des Vaters einen positiven Einfluss auf die Auswahl des Primarschultyps in Jamaika ermitteln. ${ }^{220}$ Besser ausgebildete Väter scheinen ihre Kinder eher auf die qualitativ bessere Primarschule zu schicken. Überraschenderweise konnte der Ausbildung der Mutter kein Einfluss auf die Auswahl des Schultyps nachgewiesen werden. HANDA stellte fest, dass auch bei der Auswahl des Sekundarschultyps in Jamaika nur die Ausbildung der Väter eine große Rolle spielt, während die

\footnotetext{
216 Vgl. Gertler/Glewwe (1992), S. 184.

217 Vgl. Jacoby (1994), S. 158, Birdsall (1985), S. 79-80 und Lavy (1996), S. 310-311. Das Ergebnis von Lavy ist deshalb besonders interessant, weil das Bildungsniveau der Eltern im ländlichen Ghana sehr niedrig ist, und kaum eine Mutter die Primarbildung abgeschlossen hat.

218 Vgl. Psacharopoulos/Arriagada (1989), S. 688-701.

219 Vgl. Birdsall (1985), S. 83.

220 Vgl. Glewwe/Grosh/Jacoby/Lockheed (1995), S. 243.
} 
der Mutter keinen signifikanten Einfluss hat. Auch hier entscheiden sich häufiger die besser ausgebildeten Väter für die qualitativ höherwertige High School. Diese Ergebnis überrascht, da sonst vor allem die Ausbildung der Mutter einen größeren Einfluss auf die Bildung der Kinder hat.

HANDA untersuchte außerdem die grundsätzliche Einschulungsentscheidung für die Sekundaria. Hier ist die Ausbildung der Mutter nur für Mädchen und die des Vaters nur für die Jungen signifikant. Hier zeigt sich, dass die Mütter zwar die grundsätzliche Entscheidungen über die Einschulung der Mädchen mitbestimmen, die Entscheidung, ob es sich um eine akademische Laufbahn handeln soll, aber eher in der Entscheidungsgewalt der Väter liegt.

HANDA führte seine Schätzungen einmal mit und einmal ohne Einkommensvariable durch und konnte zeigen, dass die Integration der Familieneinkommen die Koeffizienten der elterlichen Bildung zwar etwas senken, ihre Wirkungsrichtung und ihre statistische Signifikanz aber erhalten bleiben. Damit zeigt er, dass der Einfluss der elterlichen Bildung nicht ausschließlich einen Einkommenseffekt repräsentiert. Nur bei der Entscheidung die Jungen auf die High School zu schicken, verliert der Einfluss der väterlichen Bildung seine statistische Signifikanz zugunsten der Einkommensvariable. ${ }^{221}$ Bei den Jungen scheint also das Einkommen als Entscheidungsgrund auszureichen, während bei den Mädchen noch eine wohlwollende Einstellung der Väter gegenüber der Ausbildung notwendig ist.

LEIBOVICH/MAGNAC ermittelten für die Bildung beider Elternteile einen deutlichen positiven Einfluss auf die Bildung der Kinder. Differenziert nach dem Geschlecht, stellten auch sie fest, dass die mütterliche Ausbildung einen größeren Einfluss auf die Mädchen und die väterliche Bildung einen größeren Einfluss auf die Jungen hat.222 Auch DEVREYER fand einen positiven Einfluss der Ausbildung des Haushaltsvorstandes auf die abgeschlossenen Bildungsjahre der Kinder, der für die Jungen stärker ausfällt. Da 95\% der Haushaltsvorstände Männer sind, zeigt sich auch hier der stärkere Einfluss der Väter auf ihre Söhne. ${ }^{223} \mathrm{Au}$ ßerdem fand der Autor heraus, dass die Söhne männlicher Haushaltsvorstände im Schnitt mehr Bildung aufweisen als die Töchter. Weibliche Haushaltsvorstände differenzieren ihr Bildungsinvestitionen kaum nach dem Geschlecht der Kinder.

\footnotetext{
221 Vgl. Handa (1996), S. 562.

222 Vgl. Leibovich/Magnac (1996), S. 143.

223 Vgl. DeVreyer (1993), S. 72.
} 
Einige Autoren untersuchten die Auswirkungen verschiedener Bildungsstufen der Eltern auf die Ausbildung der Kinder und erhielten z. T. überraschende Ergebnisse.

BURNEY/IRFAN unterteilten die Bildung der Eltern in drei Stufen (Primary, Middle, Matric and Higher) und differenzieren die Ergebnisse nach Alter und Geschlecht des Kindes und nach Farmern und Nicht-Farmern Die Berechnungen zeigen jeweils positive Koeffizienten, die allerdings nicht für alle Fälle signifikant sind. Am deutlichsten ist noch der Effekt der höheren Bildung auf die Einschulung der Kinder. Ein eindeutiges Muster des Einflusses lässt sich allerdings nicht erkennen. ${ }^{224}$

Obwohl LAVY ermittelt hat, dass sich eine abgeschlossenen Primarausbildung der Eltern positiv auf die Ausbildung der Kinder auswirkt, kann er für ein elterliches Bildungsniveau, das über die Primarstufe hinausgeht, keinen statistisch signifikanten Einfluss mehr nachweisen. Hier ist allerdings anzumerken, dass das Bildungsniveau der Eltern im ländlichen Ghana sehr niedrig ist. Nur 7\% aller Väter und $1 \%$ aller Mütter haben ein über die Primaria hinausgehendes Bildungsniveau angegeben. ${ }^{225}$

LEIBOVICH/MAGNAC haben nachgewiesen, dass die Bildungsbeteiligung der Kinder mit dem Bildungsniveau der Eltern steigt. Allerdings haben sie auch eine Dummy-Variable für die fehlende Ausbildung der Eltern in ihre Schätzung integriert und ebenfalls einen positiven Effekt ermittelt.226

LEVISON/MOE integrierten die Ausbildung der Mutter zunächst als kontinuierliche Variable und ermittelten einen entweder nicht signifikanten oder einen signifikanten aber negativen Effekt auf die Einschulungswahrscheinlichkeit und wöchentlichen Anwesenheitsstunden. Daraufhin wurden Bildungskategorien für die Mütter gebildet. Da die Bildungsdaten der Mütter nur verfügbar waren, wenn die Mütter anwesend waren, beeinflussen sich die beiden Variablen. Zunächst ergab sich ein positiver Zusammenhang zwischen der Anwesenheit der Mutter und der Wahrscheinlichkeit, dass ein Mädchen die Schule besucht. Bei einem durchschnittlichen Bildungsniveau der Mutter, verbringt das Mädchen allerdings 10 Stunden pro Woche weniger in der Schule als ein Mädchen, dessen Mutter nicht anwesend ist. Bei der Integration von verschiedenen Bildungskategorien für die Mütter, zeigt sich ein nicht-linearer Effekt. Ein sehr niedriges oder sehr hohes Bildungsniveau der Mutter reduziert diesen negativen Effekt auf die Anwesenheit in der Schule um ca. 2 Stunden. Sehr hohe Bildungsniveaus der

\footnotetext{
224 Vgl. Burney/Irfan (1995), S. 32 und 39-40.

225 Vgl. Lavy (1996), S. 300.

226 Vgl. Leibovich/Magnac (1996), S. 139-140.
} 
Mütter reduzieren die im Haushalt geleisteten Arbeitsstunden um 2-3 Stunden wöchentlich. ${ }^{227}$ Ein deutliches Muster ließ sich aber nicht erkennen.

Obwohl die meisten Untersuchungen den positiven Einfluss des Bildungsniveaus der Eltern auf die Ausbildung ihrer Kinder belegen, geben sie kaum Aufschluss über die Wirkungsweise des familiären Bildungsniveaus. Da die meisten Autoren auch eine Einkommensvariable in ihre Schätzungen integriert haben und dennoch eine positive Wirkung des elterlichen Bildungsniveaus ermittelten, muss davon ausgegangen werden, dass die Bildungsvariable nicht nur den Einkommenseffekt repräsentiert. Fraglich ist dennoch, ob sich das Bildungsniveau vor allem über eine höhere Wertschätzung der Schulbildung oder über die bessere Unterstützung durch ausgebildete Eltern auswirken. Nur wenige Autoren integrierten eine Variable, die über die Art der Wirkungsweise Aufschluss geben könnte.

GLEWWE/GROSH/JACOBY/LOCKHEED untersuchten dazu den Einfluss der elterlichen Ausbildung auf die Grundschulerfolge (Mathematik und Lesen) der Kinder in Jamaika und konnten keinen signifikanten Einfluss feststellen. Eine mögliche Erklärung ist das relativ homogene kulturelle Niveau der jamaikanischen Gesellschaft und das relativ hohe Bildungsniveau jamaikanischer Eltern. So können auch relativ gering ausgebildete Eltern die Ausbildung ihrer Kinder wertschätzen und entsprechend unterstützen. ${ }^{228}$

BEDI/MARSHALL untersuchten den Einfluss der elterlichen Hausaufgabenhilfe auf die schulischen Erfolge der Kinder. Sie ermittelten einen vergleichsweise großen Einfluss auf die schulischen Leistungen in der zweiten und dritten Klasse. Dies kann einerseits durch das zunehmende Gewicht der Hausaufgaben und Unterrichtsvorbereitungen in höheren Klassen erklärt werden. Andererseits kommt aber auch die Wichtigkeit der elterlichen Unterstützung der Ausbildung der Kinder zum Ausdruck. ${ }^{229}$ Den Einfluss des elterlichen Bildungsniveaus haben die Autoren leider nicht untersucht.

PSACHAROPOULOS/ARRIAGADA geben in ihrer Untersuchung einen Hinweis auf die Wirkungsweise der Ausbildung der Mutter auf die Kinder. Besser ausgebildete Mütter führen zu einer höheren Einschulungswahrscheinlichkeit ihrer Kinder und zu besseren Bildungserfolgen. Ihre Berufstätigkeit hingegen beeinflusst die Einschulung positiv, die Erfolge aber negativ. ${ }^{230}$ Dieses Ergebnis zeigt, dass

227 Vgl. Levison/Moe (1998), S. 350-351.

228 Vgl. Glewwe/Grosh/Jacoby/Lockheed (1995), S. 248.

229 Vgl. Bedi/Marshall (1999), S. 665.

230 Die statistische Signifikanz für den erreichten Bildungsgrad ist das Ergebnis einer OLS Regression. Wird ein Tobit-Modell zur Berechnung herangezogen, bleibt der Koeffizient negativ ist aber nicht mehr statistisch signifikant. Vgl. Psacharopoulos/Arriagada (1989), S. 693. 
eine hohe Wertschätzung der Schule sich zwar in höheren Einschulungsraten niederschlägt, die qualifizierte Unterstützung der Kinder aber für den Bildungserfolg relevant ist.

JAMISON/LOCKHEED gingen der Frage nach, wie genau sich die „Bildungsnachfrage" über die Generationen entwickelt. Sie untersuchten dazu die Bildungsdeterminanten über drei Generationen in Nepal. ${ }^{231}$ Ihre Ergebnisse der verschiedenen Analysen können wie folgt zusammengefasst werden: Die Literacy der ersten Generation ist signifikanter Einflussfaktor für die natürlichen Fähigkeiten der zweiten Generation, die wiederum deren Schulbesuch beeinflussen. Der Schulbesuch wirkt sich positiv auf ihre Literacy, Numeracy, Reading Comprehension und Attitudinal Modernity aus. Diese Merkmale wiederum führen zu höherer Bildungsbeteiligung und besseren Bildungserfolgen der dritten Generation. Auf diese Weise wird das Ausbildungsniveau von Generation zu Generation weitergegeben und verbessert.

BURNEY/IRFAN vermuten einen starken Einfluss der Nachbarschaft einer Familie auf deren „Bildungsnachfrage“. Der ,Demonstrationseffekt" führt ihrer Meinung nach dazu, dass Haushalte, die in Gebieten mit relativ gut ausgebildeter Bevölkerung leben, mehr Bildung für ihre Kinder nachfragen werden. Sie berücksichtigen das jeweilige dörfliche Bildungsniveau in ihrer Schätzfunktion, um den Einfluss von sozialen Normen auf die Investitionsentscheidungen im ländlichen Pakistan zu ermitteln. Die Variable zeigt einen positiven Koeffizienten, der allerdings für Jungen aus Nicht-Farm-Haushalten und für Kinder über 17 Jahren nicht mehr signifikant ist. ${ }^{232}$

Außerdem wiesen JAMISON/LOCKHEED einen deutlichen positiven Effekt der Zugehörigkeit zu einer höheren Kaste auf die Bildungs- und Modernitätsvariablen für die zweite und dritte Generation nach. Diese Erkenntnis weist wieder auf einen möglichen Demonstrationseffekt einer besser ausgebildeten Nachbarschaft hin.

Das Bildungsniveau der Eltern spielt also eine große Rolle in der Bestimmung der „Bildungsnachfrage“ für die Kinder. Vor allem die Ausbildung der Mutter scheint große Relevanz zu haben. Wenn die Ergebnisse nach dem Geschlecht differenziert werden, haben die Mütter oft größeren Einfluss auf die Mädchen und die Väter auf die Söhne. Wie schon erwähnt, ist das Bildungsniveau der Eltern oft mit dem Einkommen der Familien korreliert. Da in den meisten Unter-

231 Die Autoren berücksichtigen dabei Maße für die Lesefähigkeit (Literacy), die Rechenfähigkeit (Numeracy), die angeborenen Fähigkeiten (Basic Abilities - Raven's Progressive Matrices Test) und ein Modernitätsmaß (attitudinal modernity - eine modifizierte Form der Inkeles Overall Modernity Scale). Vgl. Jamison/Lockheed (1987), S. 287 und 288.

232 Vgl. Burney/Irfan (1995), S. 27. 
suchungen aber die Einkommens- bzw. die Ausgabensituation der Haushalte berücksichtigt wurden, ist von einem Effekt der Ausbildung über den Einkommenseffekt hinaus auszugehen. Die Wirkungsweise scheint außerdem sowohl durch eine höhere Wertschätzung der Schule als auch durch die verbesserte Unterstützung der Kinder durch ihre Eltern zu resultieren. Auch ein besser ausgebildetes Umfeld scheint diese Wirkung zu haben. Welcher Art die Zusammenhänge auch sind, die Ergebnisse untermauern die These, dass besser ausgebildete Eltern mehr Bildung für ihre Kinder nachfragen und somit die Ungleichverteilung der Ausbildung fortgeschrieben wird.

\subsubsection{Geschwisteranzahl}

In den meisten Studien wird eine Variable zur Familienstruktur bei der Untersuchung der Bildungsdeterminanten berücksichtigt. Hier soll die Anzahl der Kinder genauer untersucht werden. Es ist zu vermuten, dass eine große Anzahl Kinder zu einer Reduktion der Bildungsinvestitionen in jedes einzelne Kind führt, da von einem Trade-Off zwischen der Anzahl und der ,Qualität' der Kinder ausgegangen wird. ${ }^{233}$ Wenn ein zusätzliches Kind bei gegebenem Einkommen den verfügbaren Investitionsbetrag pro Kopf senkt, werden die Bildungschancen der einzelnen Kinder negativ beeinflusst. Auch auf die Leistungen der einzelnen wird ein negativer Einfluss erwartet, da den Eltern für die Betreuung des Lernprozesses des einzelnen Kindes weniger Zeit bleibt. Andererseits ist denkbar, dass sich viele Schulkinder im Haushalt gegenseitig beim Lernen unterstützen oder auf ein bildungsfreundliches Klima im Haushalt schließen lassen. ${ }^{234}$ Um die Konkurrenz zwischen Geschwistern zu untersuchen, bietet sich vor allem das Vorhandensein bzw. die Anzahl der Geschwister im schulfähigen Alter an. Zur Untersuchung des Quantity-Quality Effekts wurde auch die Gesamtanzahl der Geschwister in die Untersuchungen integriert. ${ }^{235}$ Die folgende Tabelle stellt die Ergebnisse zusammen.

233 Vgl. zum Zusammenhang von Qualität und Anzahl der Kinder die Ausführungen in Becker/Lewis (1973) und Becker/Tomes (1976).

234 Weitere mögliche Zusammenhänge zwischen Familienstruktur und Aufgabenverteilung im Haushalt wurde bereits in Absatz 5.2.1.2 zu den Opportunitätskosten behandelt. Dieser Abschnitt beschränkt sich daher auf die Untersuchung der Anzahl der Geschwister.

235 Vgl. Bedi/Marshall (1999), S. 662 und 670, McMahon (1984), S. 89. 
Tabelle 7.5: Geschwisteranzahl als Bildungsdeterminante

\begin{tabular}{|c|c|c|c|c|c|}
\hline Autor & Land & Zielgr. & Abhängige Variable & Bildungsvar. Eltern & Ergebnis \\
\hline \multirow{2}{*}{$\begin{array}{l}\text { Gerler/ } \\
\text { Glewwe,1992 }\end{array}$} & \multirow{2}{*}{$\begin{array}{l}\text { Peru, } \\
\text { ländlich }\end{array}$} & \multirow[t]{2}{*}{$10-18 \mathrm{~J}}$. & Teilnahme Local School, j/n & Geschwister 13-17 & nicht signifikant. \\
\hline & & & Teilnahme Faraway School, j/n & Geschwister 13-17 & negativ \\
\hline \multirow{6}{*}{$\begin{array}{l}\text { Levison/Moe, } \\
1998\end{array}$} & \multirow{6}{*}{$\begin{array}{l}\text { Peru, } \\
\text { ländlich }\end{array}$} & \multirow{6}{*}{$\begin{array}{l}\text { 10-19 J., } \\
\text { nur } \\
\text { Mädchen }\end{array}$} & \multirow[t]{3}{*}{ wöchentliche Arbeitszeit i. HH } & Anzahl Mädchen 10-19 & sinkt um $1 \mathrm{~h}$ \\
\hline & & & & Anzahl Jungen 10-19 & steigt um $1 / 2 \mathrm{~h}$ \\
\hline & & & & Anzahl Kinder 7-9 & nicht signifikant. \\
\hline & & & \multirow{3}{*}{$\begin{array}{l}\text { Wahrscheinlichkeit der } \\
\text { Bildungsteilnahme }\end{array}$} & Anzahl Mädchen 10-19 & negativ \\
\hline & & & & Anzahl Jungen 10-19 & negativ \\
\hline & & & & Anzahl Kinder 7-9 & nicht signifikant. \\
\hline \multirow{4}{*}{$\begin{array}{l}\text { Jacoby, } \\
1994\end{array}$} & \multirow[t]{4}{*}{ Peru } & \multirow[t]{4}{*}{ 7-12 J. } & \multirow{4}{*}{$\begin{array}{l}\text { Wahrscheinlichkeit } \\
\text { des Rückfalls }\end{array}$} & Anzahl Geschwister 6-18 & negativ, schwach \\
\hline & & & & Anzahl Geschwister >18 & nicht signifikant. \\
\hline & & & & Altersabstand z. jüngeren Geschw. & negativ, schwach \\
\hline & & & & Altersabstand z. älteren Geschw. & nicht signifikant. \\
\hline \multirow{2}{*}{$\begin{array}{l}\text { Psach./Arria- } \\
\text { gada, } 1989\end{array}$} & \multirow[t]{2}{*}{ Brasilien } & \multirow[t]{2}{*}{ 7-14 J. } & Bildungsteilnahme, j/n & Anzahl schulpflichtiger Kinder & nicht signifikant. \\
\hline & & & Bildungserfolg, Jahre & Anzahl schulpflichtiger Kinder & OLS: negativ, Tobit: nicht signif \\
\hline \multirow{4}{*}{$\begin{array}{l}\text { Lavy, } \\
1996\end{array}$} & \multirow{4}{*}{$\begin{array}{l}\text { Ghana, } \\
\text { ländlich }\end{array}$} & \multirow[t]{4}{*}{$5-12 \mathrm{~J}}$. & jemals teilgenommen & Geschwister 6-11 & positiv \\
\hline & & & Grade Attainment & Geschwister 6-11 & positiv \\
\hline & & & jemals teilgenommen & Geschwister 12-17 & nicht signifikant. \\
\hline & & & Grade Attainment & Geschwister 12-17 & nicht signifikant. \\
\hline \multirow{2}{*}{$\begin{array}{l}\text { Bedi/Marshall, } \\
1999\end{array}$} & \multirow{2}{*}{$\begin{array}{l}\text { Honduras, } \\
\text { ländlich }\end{array}$} & \multirow{2}{*}{$\begin{array}{l}\text { Primaria, } \\
\text { 1.-3. Kl. }\end{array}$} & Anwesenheit, Tage & Anzahl Geschwister & nicht signifikant. \\
\hline & & & Testergeb. Spanisch, Mathe & Anzahl Geschwister & nicht signifikant. \\
\hline $\begin{array}{l}\text { Burney/Irfan, } \\
1995\end{array}$ & $\begin{array}{l}\text { Pakistan, } \\
\text { ländlich }\end{array}$ & $10-25 \mathrm{~J}$. & Bildungsbeteiligung, j/n & Größe des Haushalts & negativ, nur für Jungen \\
\hline \multirow{2}{*}{$\begin{array}{l}\text { Leibovich/ } \\
\text { Magnac, } 1996\end{array}$} & \multirow{2}{*}{$\begin{array}{l}\text { Kolumbien, } \\
\text { ländlich }\end{array}$} & \multirow[t]{2}{*}{$5-17 \mathrm{~J}}$. & \multirow[t]{2}{*}{ Bildungsbeteiligung } & $\varnothing$ Bildung der anderen & positiv \\
\hline & & & & $\varnothing$ Gang zur Sekundarschule & nicht signifikant \\
\hline \multirow{2}{*}{$\begin{array}{l}\text { Skyt Nielsen, } \\
1998\end{array}$} & \multirow[t]{2}{*}{ Sambia } & \multirow{2}{*}{$\begin{array}{l}\text { 7-18 J., } \\
\text { Primaria }\end{array}$} & \multirow[t]{2}{*}{ z. Z. Teilnahme, j/n } & Anzahl 7-30, aktuell in der Schule & positiv \\
\hline & & & & Rang unter den 7-30 J. & positiv \\
\hline
\end{tabular}


Die meisten Geschwistervariablen ergeben kein signifikantes Ergebnis. Die positiven und negativen Zusammenhänge halten sich ungefähr die Waage.

Gertler/Glewwe fanden für Peru nur für die entfernte Schule den erwarteten negativen Effekt. ${ }^{236}$ Dieses Ergebnis ist insofern erklärbar, als dass die Fernschule die kostspieligere Entscheidung ist und damit finanzielle Restriktionen eine größere Rolle spielen. Hier zeigt sich also, dass die Anzahl der Geschwister die Möglichkeiten einer teuren Ausbildung tatsächlich beeinträchtigen.

Ebenfalls einen Hinweis auf den erwarteten Trade-Off geben LEVISON/MOE in ihrer Untersuchung der Bildungsdeterminanten für Mädchen in Peru. Wie die Untersuchungen über die Opportunitätskosten schon zeigten, senkte die Anwesenheit von weiteren 10-19jährigen Mädchen die wöchentliche Arbeitszeit der Mädchen im Haushalt während die Jungen im gleichen Alter die Arbeitszeit der Mädchen erhöhten. Auf die Bildungsteilnahme der Mädchen haben beide Variablen allerdings einen negativen Effekt. ${ }^{237}$ Dieses Ergebnis lässt darauf schließen, dass sich Mädchen zwar die Hausarbeit teilen und sich somit gegenseitig ihre Opportunitätskosten senken können. Der negative Koeffizient bei der Bildungsbeteiligung weist aber darauf hin, dass die Konkurrenz der Geschwister um die finanziellen Ressourcen für den Schulbesuch den Opportunitätskosteneffekt sogar überkompensiert. Für Geschwister im Alter von 7-9 Jahren konnten sie allerdings keinen signifikanten Einfluss ermitteln. Die Konkurrenz scheint demnach nur zwischen gleichaltrigen Geschwistern zu wirken.

Ein ähnliches Ergebnis ermittelte JАСОВY. Er integrierte eine Variable für den Altersabstand der Schulkinder zueinander. Die Hypothese dahinter ist, dass je größer der Abstand zwischen zwei Geschwistern ist, desto weniger greifen finanzielle Beschränkungen und desto länger können sich beide Kinder der Ausbildung widmen. Er ermittelte für den Abstandsparameter zum nächst jüngeren Geschwister das erwartete negative Vorzeichen. Wenn der Altersabstand sinkt, steigt die Wahrscheinlichkeit eine Klasse zu wiederholen. Allerdings beinhaltet die Regression auch eine Variable für die Anzahl der Kinder. Mehrere schulpflichtige Kinder im Haushalt senken die Wiederholungswahrscheinlichkeit. Dieses Ergebnis deutet darauf hin, dass mehrere Schulkinder in einer Familie ihren Bildungserfolg gegenseitig positiv beeinflussen können, während ein dichter Abstand der Kinder zueinander den gegenteiligen Effekt erzeugen. Allerdings konnte er dem Abstand zum nächsten älteren Geschwister in dieser Alterskategorie keine signifikante Wirkung nachweisen. Auch die Anzahl älterer Ge-

236 Vgl. Gertler/Glewwe (1992), S. 184.

237 Vgl. Levison/Moe (1998), S. 350. 
schwister (>18) zeigte keine Wirkung. ${ }^{238}$ Der Hinweis auf einen Trade-Off ist hier also nur sehr schwach und wird von einem positiven Einfluss mehrerer Schulkinder aufeinander überlagert.

LAVY unterteilte für Ghana die Geschwistervariable nach dem Alter. Wenn er die Koeffizienten für die gesamte Stichprobe (Kinder von 5-12 Jahren) schätzt, ist nur der Einfluss der 6-11jährigen Geschwister signifikant positiv. Wird die Stichprobe auf das Alter von 9-12 Jahren begrenzt, haben nur die 12-17jährigen Geschwister einen signifikanten positiven Einfluss. ${ }^{239}$ Der Einfluss ist beim Bildungserfolg deutlicher als bei der Bildungsteilnahme. Auch diese Daten sprechen dafür, dass sich Kinder einer Altersgruppe beim Schulbesuch gegenseitig unterstützen können. Dies kann einerseits durch die Aufteilung der häuslichen Pflichten geschehen, andererseits ist es aber auch möglich, dass sich die Kinder gegenseitig bei den Hausaufgaben helfen. Der Quantity-Quality Trade-Off wird hier jedenfalls nicht bestätigt.

SKYT NiELSEN integrierte in ihre Untersuchung für Sambia die Anzahl von Geschwistern, die neben dem untersuchten Individuum die Schule besuchen. Wenn diese Variable die Einschulungswahrscheinlichkeit des Individuums erhöht, ist davon auszugehen, dass der Haushalt die Schulbildung als wichtig betrachtet. Hat die Variable allerdings einen negativen Einfluss, kann damit auch der Kosteneffekt der Schulbildung zum Ausdruck kommen und der QualityQuantity Trade-Off belegt werden. Der Koeffizient für die Anzahl von Geschwistern in der Schule ist hoch und positiv. Je mehr Kinder des Haushalts die Schule besuchen, desto größer ist die Wahrscheinlichkeit, dass auch das untersuchte Kind die Schule besuchen wird. Damit sieht die Autorin die Wirksamkeit eines bildungsfreundlichen Klimas innerhalb der Familien bestätigt. Das Ergebnis lässt darauf schließen, dass die Haushalte unterschiedliche Traditionen und Normen bezüglich der schulischen Ausbildung ihrer Kinder aufweisen und nicht ausschließlich ökonomische Anreize die Bildungsentscheidung beeinflussen. ${ }^{240}$

LEIBOVICH/MAGNAC ermittelten für Kolumbien, dass die durchschnittliche Bildung der Geschwister einen positiven Effekt auf die Bildungsbeteiligung des einzelnen hat. Auch hier scheinen die Präferenzen der Eltern für eine gleichmäßige Schulbildung der Kinder verantwortlich zu sein. ${ }^{241}$

Zusammenfassend lässt sich feststellen, dass die Ergebnisse der Untersuchungen kaum Hinweise darauf geben, dass ein deutlicher Trade-Off zwischen

\footnotetext{
238 Vgl. Jacoby (1994), S. 156-157.

239 Vgl. Lavy (1996), S. 300.

240 Vgl. Skyt Nielsen (1998), S. 14-15 und 23.

241 Vgl. Leibovich/Magnac (1996), S. 130 und 140.
} 
der Qualität und der Anzahl der Kinder bei der Bildungsentscheidung existiert. Obwohl einige Studien eine Konkurrenz um die Ressourcen bestätigen, scheinen mehrere Schulkinder einer Altersgruppe sich vielmehr gegenseitig zu unterstützen und die Eltern ein gleichmäßiges Bildungsniveau für ihre Kinder zu bevorzugen. Daher scheint die Aufteilung von Haushaltspflichten zwischen den Geschwistern und die Einstellung der Eltern zur Schulbildung viel entscheidender zu sein. 


\subsection{Charakteristika der Kinder als Bildungsdeterminanten}

Neben den Familiencharakteristika werden noch einige individuelle Charakteristika der Kinder als Einflussfaktoren der „Bildungsnachfrage“ untersucht. Im wesentlichen sind dies das Alter und das Geschlecht der Kinder. In diesem Kapitel sollen deren Wirkungen zusammengefasst werden.

\subsubsection{Alter der Kinder}

Die meisten Untersuchungen werten Daten zu Kindern im schulpflichtigen Alter aus. Grundsätzlich wäre anzunehmen, dass die Einschulungswahrscheinlichkeit und auch die absolvierten Schuljahre mit dem Alter steigen sollten. Diesen erwarteten Zusammenhang konnten nur PSACHAROPOULOS/ARRIAGADA für Brasilien und LAVY für Ghana nachweisen. ${ }^{242}$ PSACHAROPOULOS/ARRIAGADA ermittelten aber gleichzeitig eine steigende Wahrscheinlichkeit für den Schulabbruch mit dem Alter und eine höhere Wahrscheinlichkeit einer bezahlten Tätigkeit nachzugehen.

BEDI und MARSHALL ermittelten einen großen negativen und signifikanten Einfluss des Alters auf die Anwesenheit der Primarschüler in Honduras. ${ }^{243}$ Ein 9jähriger Schüler der ersten Klasse weist im Schnitt eine 3\%ige geringere Anwesenheit in der Schule auf als sein 7jähriger Klassenkamerad. Dies wird als Maß für die Opportunitätskosten bewertet. Wenn Kinder älter werden, werden sie wertvoller für andere Tätigkeiten und die Wahrscheinlichkeit, dass sie die Schule besuchen sinkt. Auf die schulischen Leistungen hat das Alter allerdings kaum Einfluss. Die Autoren schließen daraus, dass der Druck der Opportunitätskosten eher in Wiederholungen und Abbrüchen zum Ausdruck kommt. ${ }^{244}$

GERTLER/GLEWWE fanden ebenfalls einen negativen Einfluss auf die Sekundarschüler im ländlichen Peru. ${ }^{245}$ LEVISON/MOE fanden heraus, dass das Alter peruanischer Mädchen sowohl die Wahrscheinlichkeit der Bildungsbeteiligung als auch die wöchentliche Anwesenheit in der Schule signifikant senkt. 246 Und SKYT NIELSEN fand heraus, dass die jüngeren Kinder eines Haushaltes in Sambia mit größerer Wahrscheinlichkeit die Schule besuchen. ${ }^{247}$

\footnotetext{
242 Vgl. Psacharopoulos/Arriagada (1989), S. 688 und S. 693 und Lavy (1996), S. 300-304.

243 Vgl. Bedi/Marshall (1999), S. 664 bis 670.

244 Vgl. Bedi/Marshall (1999), S. 663.

245 Vgl. Gertler/Glewwe (1992), S. 183.

246 Vgl. Levison/Moe (1998), S. 350.

247 Vgl. Skyt Nielsen (1998), S. 15.
} 
DE VREYER integrierte zwar nicht das Alter in seine Schätzung der absolvierten Schuljahre in der Elfenbeinküste, aber er ermittelte einen signifikanten negativen Einfluss der Position unter den Geschwistern auf den Erstgeborenen. 248 Auch MCMAHON ermittelte einen negativen und statistisch signifikanten Effekt auf die Investitionshöhe für männliche Erstgeborene in den USA. ${ }^{249}$ Alle Autoren erklären diesen Zusammenhang mit den steigenden Opportunitätskosten mit dem Alter. Da die Kinder mit dem Alter an Erfahrung und Bildung gewinnen, werden sie für alternative Aufgaben wertvoller, was zu einem tendenziellen Rückzug aus dem Bildungssystem führen kann.

Die entgegengesetzte Wirkung, die LAVY für Ghana ermittelte, könnte dadurch erklärt werden, dass die Primarbildung in Ghana vor allem als Zugangsvoraussetzung für weitere Bildung gesehen wird und für sich genommen kaum einen Einkommen steigernden Wert aufweist.250 Je weiter seine Primarausbildung fortgeschritten ist, desto unwirtschaftlicher wird ein Abbruch. Die Abbruchentscheidung wird demnach früh fallen, da von einem zusätzlichen Jahr in der Primaria keine kompensierenden Einkommenssteigerungen zu erwarten sind.

Zusammenfassend kann also gesagt werden, dass die Bildungsbeteiligung der Kinder tendenziell mit ihrem Alter abnimmt. Der wahrscheinlichste Grund für diesen Trend kann in den steigenden Opportunitätskosten gesehen werden. Das heißt, dass die Familien einen großen Anreiz haben müssten, ihre Kinder so früh wie möglich in die Schulen zu schicken. Erstens sind die Opportunitätskosten in jungen Jahre geringer und zweitens verlängert sich die Periode, in der die Ausbildung in höhere Einkommen umgesetzt werden kann. Dieser Zusammenhang weist aber auch darauf hin, dass Kinder, die erst verspätet eingeschult werden, gleich mit einem Nachteil beginnen ${ }^{251}$, und damit tendenziell eher abbrechen und weniger Ausbildung bekommen.

\subsubsection{Geschlecht der Kinder}

Bei der Untersuchung des Einflusses des Geschlechts auf die Bildungsbeteiligung stellen einige Autoren fest, dass die Eltern weniger in die Ausbildung ihrer Töchter investieren als in die ihrer Söhne. BURNEY/IRFAN stellten eine deutlich höhere Einschulungsrate der Jungen im ländlichen Pakistan fest. Während nur

\footnotetext{
248 Vgl. DeVreyer (1993), S. 69.

249 Vgl. McMahon (1984), S. 88-89.

250 Vgl. Lavy (1996), S. 296 und die Ausführungen zu den entgangenen Einkommen (5.2.1.2.1).

251 Vgl. Bedi/Marshall (1999), S. 669.
} 
ca. 13\% der Mädchen im Alter von 10-14 Jahren eingeschult waren, besuchten immerhin fast 50\% der Jungen dieser Altersgruppe die Schule. ${ }^{252}$

GERTLER/GLEWWE zeigten für Peru, dass sowohl in der Stadt als auch auf dem Land die Jungen in allen Klassen mehr als die Hälfte der Schüler ausmachen. Den kleinsten Anteil haben die Mädchen in der Sekundarschule auf dem Land. ${ }^{253}$ Sie weisen außerdem einen negativen Einfluss des weiblichen Geschlechts auf die Bildungsentscheidung nach. Zusätzlich zeigen sie, dass die „Bildungsnachfrage“ für Mädchen stärker auf Preissteigerungen reagiert als für Jungen. Das heißt, dass ein Anstieg der Schulgebühren die Ausbildung von Mädchen eher reduziert als die der Jungen. Da für Mädchen im ländlichen Peru kaum ein Einkommensanstieg durch die Ausbildung zu erwarten ist, war ein negatives Ergebnis zu erwarten. Die Autoren verwunderte vielmehr, dass die Eltern nicht viel größere Unterschiede in der Ausbildung ihrer Söhne und Töchter machten. 254

GLEWWE/GROSH/JACOBY/LOCKHEED ermittelten allerdings einen starken positiven Zusammenhang des weiblichen Geschlechts auf die Testergebnisse im Lesen und Schreiben in jamaikanischen Grundschulen. Auf die Schulauswahl konnte allerdings kein signifikanter Einfluss des Geschlechts gezeigt werden. ${ }^{255} \mathrm{Ob}$ wohl für Mädchen also ein besserer Output zu erwarten ist, werden sie nicht häufiger auf die - eine High School-Karriere versprechende - Primarschule (im Gegensatz zur All-Age School) geschickt.

JAMISON/LOCKHEED untersuchten für Nepal zunächst auf Haushaltsniveau den Einfluss des Geschlechts auf die Ausbildung. Sie zeigten, dass je größer der Anteil der Mädchen an den schulpflichtigen Kindern ist, der Anteil der Schüler in der Familie sinkt. Sie konnten nachweisen, dass ein Teil dieses Unterschiedes daraus entsteht, dass Mädchen zur Beaufsichtigung jüngerer Geschwister gebraucht werden. Auch auf individuellem Niveau konnte diese Diskriminierung bestätigt werden. Als signifikanteste Variable auf den Bildungserfolg des Schülers hat sich sein Geschlecht erwiesen. Jungen weisen im Schnitt 3/4 mehr Bildung auf als Mädchen. Die Wahrscheinlichkeit einer Bildungsbeteiligung ist für die Mädchen 3,5-4,5 mal kleiner als für die Jungen. ${ }^{256}$

\footnotetext{
252 Vgl. Burney/Irfan (1995), S. 28. Die Zahlen beziehen sich allerdings auf das Jahr 1979. Seine eigenen Berechnungen führt er für Jungen und Mädchen getrennt durch und hat daher keine Geschlechtervariable integriert. Wie sich die einzelnen Variablen für die Geschlechter unterscheiden wird in den jeweiligen Kapiteln beschrieben.

253 Vgl. Gertler/Glewwe (1992), S. 174.

254 Vgl. die Ausführungen zu den erwarteten Nutzen der Ausbildung (5.2.2.1).

255 Vgl. Glewwe/Grosh/Jacoby/Lockheed (1995), S. 244-245.

256 Vgl. Jamison/Lockheed (1987), S. 296-297, 299, 302.
} 
Auch LAVY und SKYT NIELSEN ermittelt einen negativen Einfluss des weiblichen Geschlechts auf die Bildungsbeteiligung und die Erfolge in Ghana bzw Sambia. ${ }^{257}$. DEVREYER zeigte, dass männliche Haushaltsvorstände in der Elfenbeinküste Mädchen bei der Ausbildung diskriminieren. ${ }^{258}$

Nur PSACHAROPOULOS/ARRIAGADA berechnen für Brasilien für Jungen einen negativen Einfluss auf die Bildungsbeteiligung und den Erreichungsgrad.259 In Brasilien werden demnach mehr Mädchen eingeschult und sie erwerben ein höheres Bildungsniveau als Jungen. Die Autoren erklären diesen Zusammenhang mit höheren Opportunitätskosten für Jungen.

Einige weitere Untersuchungen konnten keinen signifikanten Effekt der Geschlechts auf die Ausbildung nachweisen. ${ }^{260}$

Die Diskriminierung von Mädchen, die in vielen Studien belegt wurde, kann mehrere Ursachen haben. Für eine ,rationale Diskriminierung' von Mädchen müssen die Eltern für sie einen niedrigeren Nettoertrag der Ausbildung erwarten. Dafür kann es verschiedene Gründe geben. Dies ist der Fall, wenn entweder die Kosten der Ausbildung von Mädchen höher sind, oder für sie niedrigere Erträge erwartet werden. Da es sehr unwahrscheinlich ist, dass für die Ausbildung von Mädchen höhere direkte Kosten anfallen als für Jungen, bleiben auf der Kostenseite nur die Opportunitätskosten als Grund für eine Ungleichbehandlung. Auf der Ertragsseite gibt es mehrere Erklärungsansätze. Erstens könnten die Eltern erwarten, dass Mädchen am Arbeitsmarkt diskriminiert werden und deshalb bei gleichem Bildungsniveau weniger verdienen werden. Zweitens könnte den Mädchen eine traditionelle Frauenrolle zugedacht werden, bei der sie sich um die Familie kümmert und nicht verdienen wird. Für diesen Fall könnten die Eltern eine knappe Grundausbildung für ausreichend halten. Drittens könnte traditionell den Jungen mehr Verantwortung bei der Unterstützung der Eltern zukommen. In diesem Fall würden die Eltern sogar bei gleichen Verdiensterwartungen mehr von der Ausbildung der Jungen profitieren. Sowohl die wirtschaftliche als auch die gesellschaftliche Wertschätzung ausgebildeter Frauen können also dazu führen, dass Mädchen weniger ausgebildet werden als Jungen. Welche Gründe hinter den beobachtbaren Zusammenhängen stecken, kann aus den Koeffizienten der Geschlechtervariablen nicht entnommen werden. Allerdings haben einige Autoren ihre Schätzungen für Jungen und Mädchen getrennt durchgeführt. Die Ergebnisse wurden im Absatz über die Nutzen und Kostenfaktoren der Ausbildung bereits dargestellt.

\footnotetext{
257 Vgl. Lavy (1996), S. 300-304 und Skyt Nielsen (1998), S. 17

258 Vgl. DeVreyer (1993), S. 68-69.

259 Vgl. Psacharopoulos/Arriagada (1989), S. 688 und S. 693.

260 Vgl. z.B. Bedi/Marshall (1999), Jacoby (1994) oder Leibovich/Magnac (1996).
} 


\subsection{Zusammenfassung der familiären und individuellen Merkmale}

Alle empirischen Untersuchungen verwenden eine Variable zur Einkommenssituation der Haushalte in ihre Schätzung. Wie die obigen Ausführungen zeigten, wurde in den meisten Untersuchungen ein positiver Einfluss der unterschiedlich 
besondere Relevanz gerade die Bildungshindernisse armer Familien aufzudecken, um ihre Teilnahme am Bildungssystem zu gewährleisten und damit den Teufelskreis zu durchbrechen.

Der in der Theorie immer wieder untersuchte Trade-Off zwischen der Anzahl der Kinder und ihrem Bildungsniveau konnte in der Empirie nicht nachgewiesen werden.

Entgegen den Erwartungen hat das Alter der Kinder einen negativen Einfluss auf ihre Schulbildung, was durch steigende Opportunitätskosten erklärt wird. Die meisten Studien zeigen außerdem eine Diskriminierung der Mädchen. Weder das Alter noch das Geschlecht der Kinder wird mit den Einkommensverhältnissen korreliert sein. Sind aber z.B. die Opportunitätskosten oder schlechtere Bedingungen am Arbeitsmarkt der Grund für eine Diskriminierung der Mädchen oder für ein relativ frühes Abbrechen der Ausbildung, kann auch hier durchaus von einer größeren Relevanz für arme Haushalte ausgegangen werden. ${ }^{263}$ Auch Traditionsbewusstsein und das Festhalten an alten Strukturen kann mit dem Einkommensniveau variieren. Welche Zusammenhänge hinter den Beobachtungen stecken, gehen aus den Untersuchungen nicht hervor.

263 Vgl. dazu die Ausführungen zu den Opportunitätskosten in Kapitel 5.3. 


\section{Zusammenfassung der Erkenntnisse empirischer Studien}

Wie die Zusammenfassung der empirischen Untersuchungen zur „Bildungsnachfrage" in Entwicklungsländern gezeigt hat, handelt es sich in den meisten Fällen streng genommen nicht um Nachfrageschätzungen. Vielmehr wurde der Einfluss zahlreicher Determinanten auf unterschiedlich definierte Bildungsindikatoren untersucht. Dieses Vorgehen ist notwendig, weil eine stringente Ableitung der empirischen Schätzgleichung aus der Theorie nicht möglich ist. Zum einen lässt sich keine Variable direkt beobachten, die die Bildungsnachfrage ausdrücken könnte. Zum anderen muss davon ausgegangen werden, dass nicht ausschließlich ökonomische Gründe die Bildungsentscheidung beeinflussen. Wenn die Nutzenmaximierung nicht das alleinige Ziel der Ausbildung darstellt, können keine eindeutigen Wirkungszusammenhänge für alle Einflussfaktoren angenommen werden. Viele der möglichen Einflussfaktoren sind außerdem nicht direkt beobachtbar. Die Ableitung überprüfbarer Hypothesen aus der Theorie wird damit erschwert.

Um dennoch ein wenig Licht in die Bildungsentscheidungen zu werfen, wurden viele verschiedene beobachtbare Variablen in die Schätzungen integriert, von denen man annimmt, dass sie einen Hinweis auf die Wirkungszusammenhänge geben könnten. Die Interpretation der Ergebnisse hat dann mit der entsprechenden Vorsicht zu erfolgen. Die Sichtung und Einordnung der umfassenden und vielschichtigen Untersuchungsergebnisse und ihre aufwendige Auswertung und Zusammenfassung war notwendig, um dennoch Untersuchungsmuster aufzeigen zu können.

Nur für die Nutzen- und Kostenfaktoren lassen sich eindeutige Wirkungszusammenhänge aus der Theorie ableiten. Danach müssten höhere Kosten der Ausbildung die Bildungsnachfrage senken und höhere Nutzen die Nachfrage erhöhen. Die Kostenhypothese lässt sich anhand der Untersuchungen für die Opportunitätskosten bestätigen, obwohl auch sie oft nur durch Hilfsindikatoren abzubilden waren. Der Zusammenhang zwischen den erwarteten Nutzen der Ausbildung und der „Bildungsnachfrage“ bleibt aufgrund der Datenlage nahezu unerforscht.

In der bildungsökonomischen Literatur herrscht Konsens, dass nicht nur Nutzen und Kosten die Bildungsinvestitionen beeinflussen, sondern auch in den Familien selbst die Ursache für das Investitionsverhalten begründet liegen kann. Alle Autoren integrierten sozioökonomische Charakteristika in ihre Schätzungen. Für die meisten Indikatoren wurden recht einheitliche Ergebnisse ermittelt. Der tatsächliche Wirkungszusammenhang konnte oft allerdings nur vermutet werden. 
Ob sich diese Zusammenhänge wieder auf Kosten- und Nutzenwirkungen zurückführen lassen oder auf Traditionen und Einstellungen beruhen blieb unklar. Auch ob Erwartungen, Präferenzen und Traditionen zwischen Bevölkerungsgruppen variieren, wurde nicht untersucht. 


\section{Teil: Auswertung der eigenen Haushaltsbefragung}

\section{Grundlagen zur eigenen Haushaltsbefragung}

Um die Partizipation armer Haushalte am Bildungsangebot Perus zu untersuchen, wurde für die Hauptstadt Lima im September 1997 eine Haushaltsbefragung über die Ausbildung der Kinder durchgeführt. Obwohl die Landbevölkerung oft ärmer ist als die Stadtbewohner, wurde die Stadtbevölkerung als Zielgruppe gewählt, weil hier die Einkommensdifferenzen sehr hoch sind und daher ein Vergleich von armen und reichen Haushalten besser durchführbar ist. Wie die Auswertungen der empirischen Arbeiten gezeigt hat, sind die Nachfrageelastizitäten in Städten höher und variieren auch stärker zwischen den Einkommensgruppen. Außerdem leben hier Haushalte mit unterschiedlichen Einkommen sehr eng beieinander und sehen sich theoretisch dem gleichen Bildungsangebot gegenüber. Nur so kann ein Vergleich der Verhaltensweisen erfolgen.

Mit dieser Untersuchung wird versucht, den Mechanismus für die Beteiligung an der Basisausbildung aufzudecken und damit die Lücke im Forschungsstand ein wenig zu verkleinern.

Um die folgenden Untersuchungsergebnisse besser einordnen zu können, erfolgt hier zuerst eine kurze Darstellung des peruanischen Bildungssystems. Anschließend wird die Bevölkerungsstruktur Limas und die daraus folgende Auswahl der Stichprobe dargestellt.

\subsection{Das peruanische Bildungssystem}

Die peruanische Ausbildung hat in den letzten Jahren erstaunliche Erfolge erzielt. Die Primarausbildung erreicht heute nahezu alle Kinder. Und 80\% der 1216jährigen Kinder besuchen die Sekundarschule. Damit erreichte Peru eine deutlich höhere Partizipationsrate als die meisten Länder der gleichen Einkommensstufe. Diese Ergebnis erscheint noch erstaunlicher, wenn man die Anteile der Bildungsausgaben der Länder vergleicht. Hier lag Peru 1997 mit einem Anteil von 3\% deutlich unter dem lateinamerikanischen Durchschnitt von 4,5\%.264 Die hohe Einschulungsrate konnte einerseits dadurch zustande kommen, dass die Peruaner der Ausbildung einen relativ hohen Wert beimessen. Ihr privater Anteil an der Bildungsfinanzierung war 1997 relativ hoch und summierte sich

264 Vgl. Weltbank (2001), S. 15. 
insgesamt auf 2\% des GDP.265 Allerdings zeigt eine genauere Betrachtung der peruanischen Ausbildung, dass die hohen Einschulungsraten auf Kosten der Qualität der Ausbildung erreicht wurden. ${ }^{266}$

Das peruanische Bildungssystem besteht aus vier Stufen: inicial, primaria, secundaria und superior. Die Vorstufe, die Primaria und die Sekundaria sind obligatorisch und werden im peruanischen Bildungssystem als Basisausbildung bezeichnet. Die vier Stufen werden im folgenden kurz vorgestellt.

Die Educación Inicial ist die erste Stufe des formalen Bildungssystems. Konform mit dem schon hundert Jahre alten Wissen, dass die ersten 5 Lebensjahre die Grundlage für die Entwicklung eines Menschen legen, erklärte die peruanische Verfassung den Besuch einer vorschulischen Einrichtung für die 0-5Jährigen als verpflichtend.267 Das Angebot für die 0-2jährigen besteht in den Nidos (estimulación temprana) und für die von 3-5jährigen in den Jardines (aprestamiento para la lectoescritura). ${ }^{268}$ Auch außerschulische Einrichtungen spielen hier eine Rolle. Von einer flächendeckenden Bereitstellung dieser Bildungseinrichtung und einer umfassenden Beteiligung aller Bevölkerungsschichten kann allerdings bei weitem nicht gesprochen werden.

Die Educación Primaria bildet die zweite Stufe des formalen Bildungssystems. Sie bildet mit 70\% aller Matrikulationen die breite Basis der Bildungspyramide, was bedeutet, dass ein großer Teil der Bevölkerung über diesen Bildungsstand nicht hinaus kommt. Die Primaria ist somit ein unverzichtbares Mittel zur Integration auch der ärmsten Sektoren in die moderne Gesellschaft. 269

Sie richtet sich an Kinder ab dem 6. Lebensjahr und dauert 6 Jahre. Sie hat zum Ziel, eine angemessene Grundlage zu vermitteln bezüglich der Lesefähigkeit, des mündlichen und schriftlichen Ausdrucks, der elementaren Mathematik, der historisch-sozialen Aspekte Perus und der Welt und der grundlegenden Ereignisse der Natur. Sie wird auch für bisher nicht ausgebildete Erwachsene angeboten. Die peruanische Realität zeigt, dass sich in der Primaria für Kinder eine beachtliche Anzahl Schüler befinden, die älter als 11 Jahre sind. ${ }^{270}$

\footnotetext{
265 Vgl. Weltbank (2001), S. 24.

266 Vgl. Weltbank (2001), S. 29-45.

267 Über die Einbeziehung der Vorstufe in die Basisausbildung bestand bisher kein Konsens. Je nach Auslegung der peruanischen Verfassung (Art.17) konnte die Schulpflicht zwischen 12 bis 16 Jahre variieren. Vgl. Quánto S.A. (1993), S.44 und Díaz Díaz (1994), S. 42. Im Rahmen der laufenden Bildungsreform soll ein Jahr Vorschule eindeutig zum Pflichtbestandteil der Basisausbildung erklärt werden.

268 Vgl. dazu Foro Educativo (1997b), S. 41 und Ministerio de Educación (1995), S. 12.

269 Vgl. Foro educativo (1997c), S. 97.

270 Vgl. Ministerio de Educación (1995), S. 12.
} 
Die Educación Secundaria hat zum Ziel, die wissenschaftliche und humanistische Ausbildung der Primaria zu vertiefen, Werte zu erhalten, eine berufliche Orientierung zu geben und die Schüler auf verschiedenen Gebieten (cienciashumanidades y áreas técnicas) weiterzubilden. ${ }^{271}$ Sie richtet sich an Kinder zwischen 12 und 16 Jahren. Die derzeitige Sekundarbildung erstreckt sich über 5 Jahre und ist in zwei Zyklen gegliedert. ${ }^{272}$ Die ersten 2 Jahre sind allgemeiner und grundlegender Art und haben einen gemeinsamen Lehrplan für alle Schüler. Der zweite Zyklus von 3 Jahren bietet verschiedene Bildungsalternativen zur Wahl. Im ersten Zyklus sollen die Schüler - neben der Vertiefung ihrer Grundbildung - ihre Interessen, Fähigkeiten und beruflichen Neigungen entdecken. Im zweiten Zyklus sollen sie dann in der Lage sein, sich für eine wissenschaftliche, humanistische Laufbahn oder eine technische Ausrichtung zu entscheiden.

Die Schulpflicht endet nach der Sekundaria mit dem Abschlusszeugnis der Basisausbildung. Ohne die Berücksichtigung vorschulischer Einrichtungen dauert sie 11 Jahre. Der Bildungsweg kann hier abgeschlossen oder mit einer höheren Ausbildung (der educación superior) fortgeführt werden.

Die Educación Superior bildet die vierte Stufe der peruanischen Ausbildung und beinhaltet eine nicht-universitäre und eine universitäre Ausbildung. Die nichtuniversitäre Ausbildung untersteht dem Bildungsministerium und beinhaltet die Formación Magistral, die Educación Superior Tecnológica und die Educación Artística. Die Zugangsvoraussetzung zu diesen Einrichtungen besteht in der abgeschlossenen Sekundarbildung. ${ }^{273}$ Der Zugang zu den Universitäten wurde bisher durch spezielle auf die Universitäten vorbereitenden Prüfungen geregelt.

Das peruanische Bildungssystem wird seit 1982 durch das Ley General de Educación geregelt. Die Verabschiedung der Verfassung Ende 1993 hat die Debatte über die Notwendigkeit einer Bildungsreform angeregt.

Die Bemühungen um eine Bildungsreform lassen sich in zwei Perioden einteilen. Von 1990 bis 1994 beschränkte sich die Politik darauf, den Staatsapparat zu reduzieren und die schulische Infrastruktur zu verbessern. Die ersten Jahre, die mit den Maßnahmen der Strukturanpassung einhergingen, zeichneten sich ausschließlich durch eine Verminderung der Bildungsausgaben und die Reduktion des Bildungsministeriums aus. Während der ersten Jahre der Regierung Fujimori stellte das Thema Bildung einen relativ unbeachteten und undefinierten

271 Vgl. Foro Educativo (1997a), S. 75-77. Hier wird die Realität des Bildungssystems einer kritischen Würdigung unterzogen.

272 Vgl. Weltbank (2001), S. 85.

273 Vgl. Ministerio de Educación (1995), S. 13-14. 
Bereich dar. Von 1990 bis 1994 durchliefen 7 Minister das Bildungsministerium. Die wechselnden Verordnungen und Maßnahmen, die eingeführt und wieder annulliert wurden, destabilisierten die Bildungspolitik noch zusätzlich. Erst 1993 begannen die Bildungsausgaben wieder zu steigen, konzentrierten sich aber auf die Konstruktion von Schulen und Klassenzimmern. 1994 hat die peruanische Regierung ein weites Programm zum Schulbau initiiert. Im selben Jahr wurde im Durchschnitt jeden Tag eine neue Schule eröffnet.274 Im März 1997 wurde der Ausbau der Infrastruktur der Schulen für abgeschlossen erklärt. Die physischen Voraussetzungen im Erziehungswesen seien jetzt vorhanden, so der offizielle Tenor. ${ }^{275}$

Erst 1995 trat eine Wende ein und die Regierung deklarierten die Bildung zur Priorität. Seitdem wurde die Bildungsdiskussion auf qualitative Ziele ausgeweitet.276 Obwohl seit 1995 einige Veränderungen in Bewegung gesetzt wurden, kann man nicht von einer Reform im Sinne eines 'globalen und systematischen Planes' sprechen wie z.B. in Chile oder Bolivien. ${ }^{277}$ Vielmehr handelt es sich um einzelne Maßnahmen, die nicht immer aufeinander abgestimmt waren. Zweifelsohne waren alle Bemühungen zur Verbesserung der Situation für die Bildungsnachfrager gedacht. Aber die Ansprüche wurden nicht immer von einer Praktikabilitätsprüfung begleitet und scheiterte deshalb häufig an Problemen der Durchführung. 278

Erst 1997 wurde von der Regierung eine integrale Reform des Erziehungswesens angekündigt ${ }^{279}$, deren Durchsetzung noch andauert. Da die Daten auf der Grundlage der oben beschriebenen Bildungsstruktur erhoben wurden, werden die Reformpläne der Vollständigkeit halber nur genannt.

Die Reform sieht vor, dass alle Schüler ab fünf Jahre mindestens ein Jahr einer vorschulischen Ausbildung absolvieren müssen und die Sekundaria von fünf auf vier Jahre reduziert wird. Damit erstreckt sich die Basisausbildung inklusive der Vorschule auf 11 Jahre. Die Verkürzung der Sekundaria soll durch die Einführung eines zweijährigen Kurssystems (Bachillerato) kompensiert werden. Im Rahmen dieser Ausbildung sollen die Schüler sowohl auf die universitäre Ausbildung als auch auf den Arbeitsmarkt vorbereitet werden. Sie gehört nicht zur Schulpflicht, wird aber an öffentlichen Schulen kostenlos angeboten. ${ }^{280}$ Ab-

\footnotetext{
274 Vgl. Díaz Díaz (1994), S. 51.

275 Vgl. KAS Kurzinfo, April 1997.

276 Zu den Zielen vgl. Foro Educativo (1997c), S. 13.

277 Vgl. Foro Educativo (1997c), S. 105.

278 Vgl. Díaz Díaz (1994), S. 41-42.

279 Vgl. Ministerio de Educación (1997).

280 Vgl. Weltbank (2001), S. 6.
} 
schlusszertifikate können dann nach der Basisausbildung, dem Bachillerato und wie vorher nach Abschluss einer weiteren Ausbildung erworben werden.

\subsection{Die Bevölkerungsstruktur Limas und die Stichprobenauswahl}

Die Bevölkerung Limas wird in Volkszählungen üblicherweise in vier Schichten eingeteilt. Für diese Einteilung werden die folgenden sozioökonomischen Kriterien herangezogen: Geburtsort, Bildungsniveau und Beruf des Haushaltsvorstandes, das Haushaltseinkommen und die Wohnsituation der Familie. ${ }^{281}$ Die Informationen, die zu einer derartigen Einteilung notwendig waren, wurden aus vorangegangenen Volkszählungen und Haushaltsbefragungen entnommen.

Die Tabelle zeigt die Verteilung der Bevölkerung auf diese vier Schichten.

Tabelle 9.1: Bevölkerungsverteilung, Lima 1997

\begin{tabular}{|l|rrrr|}
\hline \multirow{2}{*}{ Gesamt } & \multicolumn{4}{|c|}{ Schichten } \\
\cline { 2 - 5 } & A & B & C & D \\
\hline 6.674 .809 & 251.232 & 1.080 .103 & 2.351 .758 & 2.991 .716 \\
$100 \%$ & $4 \%$ & $16 \%$ & $35 \%$ & $45 \%$ \\
\hline
\end{tabular}

Quelle: Perú en Números 1996, Cuánto S.A., Berechnungen: Cuánto S.A.

Die mit A gekennzeichnete Schicht weist ein hohes sozioökonomisches Niveau auf und die mit D gekennzeichnete Schicht ein sehr niedriges. Diese Bezeichnung wird in den folgenden Ausführungen beibehalten.

Nur 4\% der limenser Bevölkerung gehören der obersten Schicht an, während $45 \%$ zu der untersten Schicht gehören. Für die Aufteilung der Stichprobe auf die vier Schichten hatte das zur Folge, dass die oberste Schicht in der Stichprobe überrepräsentiert ist, da ihre Anzahl Haushalte bei einer Stichprobengroße von 800 sonst zu klein ausgefallen wäre. Die Auswahl der Stichprobe ist im Anhang dokumentiert. ${ }^{282}$

Da diese Studie die Teilnahme der Haushalte an der Basisausbildung untersuchen sollte, mussten außerdem die Haushalte mit Kindern im schulfähigen Alter identifiziert werden. Dazu wurde die Bevölkerung nach Haushalten gruppiert und nach der Anzahl der Kinder pro Haushalt differenziert. Die Tabelle für die Verteilung der Haushalte auf die vier Schichten gibt ein leicht verändertes Bild

281 Die in den einzelnen Schichte dominierenden Ausprägungen dieser Kriterien können der Tabelle A 1 im Anhang entnommen werden. Außerdem liegen detaillierte Aufstellung der Bevölkerung nach Schichten und Armutsindizes für alle Distrikte Limas vor. Sie wurden zur Auswahl der Stichprobe herangezogen und können ebenfalls dem Anhang entnommen werden (Tabelle A2-A3).

Die Verteilung der Haushalte auf die Stichprobe nach ihren tatsächlichen Bevölkerungsanteilen ist in Tabelle A4 wiedergegeben. Die Tabelle A5 gibt die notwendige Abweichung von der tatsächlichen Verteilung an. Sie bildete die Grundlage für die Befragung von 800 Haushalten. Die darauf basierenden tatsächlichen Befragungsergebnisse lagen dann für 801 Haushalte vor. Die Anzahl Haushalte für die einzelnen Schichten weicht von der Tabelle geringfügig ab, da einzelne Haushalte nach einer Überprüfung ihrer Schichtzugehörigkeit nachträglich einer anderen Gruppe zugeordnet werden mussten. 
als die der Bevölkerungsanzahl, da die Haushalte der unteren Schichten im Schnitt größer sind als die der oberen.

Tabelle 9.2: Haushalte mit Kindern im schulfähigen Alter, Lima 1997

\begin{tabular}{|l|r|rrrr|}
\hline Anzahl Kinder & Gesamt & \multicolumn{3}{|c|}{ Schichten } & C \\
\cline { 3 - 6 } & & $\mathrm{A}$ & $\mathrm{B}$ & $\mathrm{D}$ \\
\hline gesamt & $100 \%$ & $4 \%$ & $19 \%$ & $37 \%$ & $40 \%$ \\
0 & $35 \%$ & $60 \%$ & $50 \%$ & $34 \%$ & $27 \%$ \\
Untergesamtheit & $100 \%$ & $100 \%$ & $100 \%$ & $100 \%$ & $100 \%$ \\
$1-2$ & $72 \%$ & $94 \%$ & $82 \%$ & $75 \%$ & $66 \%$ \\
3 & $16 \%$ & $2 \%$ & $14 \%$ & $15 \%$ & $19 \%$ \\
4 und mehr & $11 \%$ & $2 \%$ & $4 \%$ & $10 \%$ & $15 \%$ \\
\hline
\end{tabular}

Quelle: Perú en Números 1996, Cuánto S.A., Berechnungen: Cuánto S.A.

Für die Befragung wurden nur Haushalte ausgewählt, die mindestens ein Kind im schulfähigen Alter hatten (also aus der Untergesamtheit stammen). Damit kamen aus der obersten Schicht $60 \%$ der Haushalte für die Befragung nicht in Frage. In der untersten Schicht sind dies nur 27\%. Die Befragungsergebnisse beziehen sich damit nur auf die verbleibenden Familien mit Kindern im schulpflichtigen Alter. Dies muss bei der Interpretation berücksichtigt werden.

Insgesamt wurden für 801 Haushalte Daten zur sozioökonomischen Charakterisierung und subjektiven Wahrnehmung erhoben. ${ }^{283}$ Für alle Kinder im schulpflichtigen Alter wurden ausführliche Daten über ihre Bildungssituation erfragt. Daraus entstand eine Stichprobe von ca. 1.500 Kindern zwischen 6 und 16 Jahren.

Die Beantwortung der Fragen erfolgte in den meisten Fällen durch die Mütter der Kinder. In den wenigen Fällen, in denen der Vater oder ein anderes erwachsenes Mitglied des Haushaltes die Antworten gab, hatte diese Person auch angegeben, die Bildungsentscheidungen für die Kinder (mit) zu treffen.

283 Die Daten auf Haushaltsniveau können nicht einfach auf die Gesamtbevölkerung Limas übertragen werden. Erstens ist die oberste Bevölkerungsschicht in der Stichprobe überrepräsentiert und zweitens wurden nur Familien mit Kindern in die Befragung einbezogen. Im Anhang ist die Berechnung von Expansionsfaktoren dokumentiert, mit denen die Befragungsergebnisse für Gesamtlima erweitert werden können (Tabelle A6-A8). Für die Aussagen dieser Arbeit ist eine Erweiterung der Daten nicht sinnvoll, da sie sich auf die Verhältnisse von Schulkindern beschränken sollen. 


\section{Beschreibung der sozioökonomischen Schichten}

Zur Charakterisierung der sozioökonomischen Schichten wurden Fakten zur familiären Situation und der Ausbildung der Kinder erhoben. Diese werden im folgenden zusammengetragen, um ein detailliertes Bild einer für jede Schicht typischen Familie zu zeichnen. Bei diesen Daten auf Haushaltsniveau muss wieder berücksichtigt werden, dass es sich bei der Stichprobe nur um Familien mit schulpflichtigen Kindern handelt. Alle folgenden Tabellen stellen Daten aus der eigenen Stichprobe dar.

\subsection{Sozioökonomische Charakterisierung der Schichten}

Wie schon erwähnt, erfolgte die Einteilung der sozioökonomischen Schichten nach dem Geburtsort des Haushaltsvorstandes, seiner Ausbildung und Beschäftigung, dem Familieneinkommen und der Wohnverhältnisse als zusätzlichen Indikator für die ökonomische Situation der Haushalte. ${ }^{284}$ Hier fällt auf, dass auch genau diese Haushaltscharakteristika in den empirischen Untersuchungen auf ihre Relevanz für die Ausbildung der Kinder untersucht wurden. In den folgenden Ausführungen werden die zur Einteilung der Schichten verwendeten Charakteristika und deren Ausprägungen in den einzelnen Schichten der eigenen Stichprobe dargestellt. Über diese Einteilungskriterien hinaus, lieferte die Befragung noch weitere Informationen, die ein differenzierteres Bild der Ausprägungen zulassen. Da diese zur Erklärung von Bildungsverhalten relevant sein könnten, werden auch sie im folgenden dargestellt. Die folgende Darstellung dient also der Charakterisierung der Schichten der eigenen Stichprobe. Die Daten bilden den Hintergrund für die Auswertung der Bildungsdaten für die Kinder und der Erwartungswerte der Eltern, die anschließend erfolgt.

\subsubsection{Haushaltsstruktur}

Die Haushaltstruktur geht zwar nicht in die Einteilung der Schichten ein, aber sie wurde in den empirischen Untersuchungen häufig als bildungsrelevant dargestellt. Deshalb soll zunächst ein Überblick über die Größe und die Haushaltsvorstände 285 gegeben werden.

\footnotetext{
284 Leider liegen keine genauen Grenzen und Gewichtungen der Kriterien zur Einteilung der Bevölkerung auf die vier Schichten vor. Bei Interesse kann dazu das limenser Forschungsinstitut Cuánto angeschrieben werden.

285 Bei der Person des Haushaltsvorstandes handelt es sich um das Familienmitglied, welches im Haushalt die Entscheidungen trifft und den weiteren Mitgliedern als Vorbild dient. Es ist nicht notwendig, dass diese Person auch das höchste Einkommen im Haushalt verdient.
} 
Beim Lesen der folgenden Tabellen muss berücksichtigt werden, dass die Stichprobe nur aus Haushalten mit mindestens einem Kind im schulpflichtigen Alter besteht. Wie die obige Tabelle zeigt, mussten dazu 35\% der Familien ausgegrenzt werden. Da es sich bei der Ausgrenzung vor allem um kinderlose Familien handelt, sind die folgenden Daten zur Familiengröße nach oben verzerrt²86 und damit nicht auf Gesamt-Lima übertragbar. ${ }^{287}$ Dies ist für die Untersuchung auch nicht notwendig. Vielmehr wurde die Eingrenzung beabsichtigt, um die Familienverhältnisse aufzeigen zu können, in denen limenser Schulkinder aufwachsen.

Tabelle 10.1: Familienstruktur der Stichprobe

\begin{tabular}{|l|c|cccc|}
\hline Familienstruktur & Stichpr. & \multicolumn{4}{|c|}{ Sozioökonomische Schichten } \\
HH mit Kindern & gesamt & A & B & C & D \\
\hline$\varnothing$ Größe $d . H H$ & 5,3 & 4,7 & 4,9 & 5,2 & 5,7 \\
\hline 1 Kind & $25 \%$ & $37 \%$ & $35 \%$ & $24 \%$ & $17 \%$ \\
2-3 Kinder & $60 \%$ & $59 \%$ & $60 \%$ & $64 \%$ & $57 \%$ \\
4 oder mehr Kinder & $15 \%$ & $7 \%$ & $6 \%$ & $12 \%$ & $26 \%$ \\
\hline$\varnothing$ Anzahl Kinder (0-18) & 2,4 & 2,0 & 2,0 & 2,3 & 2,8 \\
\hline
\end{tabular}

Wie die Daten zeigen, besteht der durchschnittliche Haushalt mit Schulkindern der untersten Schicht aus genau einer Person mehr als der durchschnittliche Haushalt der obersten Schicht. Die Unterschiede in der Familiengröße scheinen aus der unterschiedlichen Anzahl Kinder288 zu resultieren. Deutliche Unterschiede in der Anzahl der Erwachsenen pro Familie sind nicht nachzuweisen. Arme Großfamilien, in denen Tanten, Onkel und Großeltern zusammenleben, scheinen in Lima an Gewicht verloren zu haben.

Die meisten Kinder im schulpflichtigen Alter hatten zur Zeit der Umfrage ein oder zwei minderjährige Geschwister. Allerdings hat ein Viertel der Familien der untersten Schicht 4 oder mehr minderjährige Kinder, während dies in den oberen Schicht kaum vorkommt. Dafür wachsen in mehr als einem Drittel der Haushalte der oberen Schichten Einzelkinder auf.

80\% aller Kindern leben in Haushalten, in denen beide Elternteile anwesend sind. Alleinerziehende Väter sind in allen Schichten relativ selten. Nur 3\% der Kinder werden allein von ihren Vätern erzogen. Deutliche Unterschiede zwischen den Schichten sind nicht zu erkennen.

286 Die Verzerrung ist für die oberste Schicht am stärksten, da hier 60\% der Familien keine Schulkinder aufweisen, während nur $27 \%$ der der untersten Schicht aus der Untersuchung ausgeklammert blieben.

287 Vgl. hierzu noch mal FN 283.

288 Bei der Anzahl der Kinder handelt es sich um alle im Haushalt lebenden Kinder der entsprechenden Altersgruppe, unabhängig von deren Verwandtschaftsverhältnis zum Haushaltsvorstand. In den insgesamt 801 befragten Haushalten befanden sich 1.807 Kinder unter 18 Jahren. 19,7\% davon hatten das 6 Lebensjahr noch nicht abgeschlossen. Die Stichprobe liefert demnach Daten für ca. 1.500 Kindern zwischen 6 und 18 Jahren. 
Die folgende Tabelle gibt einige weitere Merkmale der Haushaltsvorstände an.

Tabelle 10.2: Charakteristika der Haushaltsvorstände

\begin{tabular}{|l|c|cccc|}
\hline Charakterisierung & Stichpr. & \multicolumn{4}{|c|}{ Sozioökonomische Schichten } \\
HH-Vorstand & gesamt & A & B & C & D \\
\hline$\varnothing$ Alter & $43 \mathrm{~J}$. & $44 \mathrm{~J}$. & $43 \mathrm{~J}$. & $44 \mathrm{~J}$. & $41 \mathrm{~J}$. \\
\hline Männlich & $86 \%$ & $85 \%$ & $86 \%$ & $84 \%$ & $89 \%$ \\
\hline Weiblich & $14 \%$ & $15 \%$ & $14 \%$ & $16 \%$ & $11 \%$ \\
$\quad$ davon alleinerziehend & $87 \%$ & $75 \%$ & $96 \%$ & $88 \%$ & $84 \%$ \\
\hline
\end{tabular}

Der durchschnittliche Haushaltsvorstand ist männlich und 43 Jahre alt. In der untersten Schicht sind die Haushaltsvorstände im Schnitt etwas jünger und sogar noch häufiger männlich als in den höheren Schichten. In den wenigen Haushalten, denen eine Frau vorsteht, sind dies vor allem allein erziehende Mütter.

Da die Betreuung der Kinder traditionell Aufgabe der Frauen ist, wurde zusätzlich gefragt, wer im Haushalt die Bildungsentscheidungen für die Kinder trifft. Die Tabelle fasst die Ergebnisse zusammen.

Tabelle 10.3: Wer trifft die Bildungsentscheidung

\begin{tabular}{|l|c|cccc|}
\hline Wer trifft die & Stichpr. & \multicolumn{4}{|c|}{ Sozioökonomische Schichten } \\
Bildungsentscheidung & gesamt & A & B & C & D \\
\hline Vater & $8 \%$ & $5 \%$ & $6 \%$ & $8 \%$ & $9 \%$ \\
Mutter & $25 \%$ & $16 \%$ & $26 \%$ & $26 \%$ & $26 \%$ \\
beide Elternteile & $66 \%$ & $79 \%$ & $66 \%$ & $64 \%$ & $63 \%$ \\
eine dritte Person & $2 \%$ & - & $2 \%$ & $2 \%$ & $2 \%$ \\
\hline
\end{tabular}

Obwohl in $86 \%$ der Haushalte ein Mann den Vorstand bildet, gaben $66 \%$ der Familien an, dass beide Elternteile gemeinsam die Bildungsentscheidung treffen. In der obersten Schicht sind dies deutlich mehr, in der untersten Schicht etwas weniger Familien. In den restlichen Familien ist es vor allem die Mutter, die die Entscheidungen für ihre Kinder trifft. Die empirischen Untersuchungen haben gezeigt, dass die Ausbildung der Mütter einen größeren Einfluss auf die Ausbildung der Kinder hat als die Ausbildung der Väter. Daraus könnte geschlossen werden, dass sie auch einen größeren Einfluss auf die Entscheidung haben. Die oben genannten Antworten decken sich mit diesem Muster. Im folgenden soll daher die Herkunft und die Schulbildung für beide Elternteile dargestellt werden, obwohl die Kriterien zur Schichteinteilung nur die Charakteristika der Haushaltsvorstandes berücksichtigen. 


\subsubsection{Herkunft der Familie}

Aus der Herkunft der Familien lassen sich z.B. verschiedene Normen, Traditionen, oder Klassenwerte erklären. Neben dem für die Schichteinteilung relevanten Geburtsort des Haushaltsvorstandes werden hier noch einige weitere Hinweise auf die Herkunft der Familien dargestellt. Als ein erster Hinweis auf die ethnische Abstammung der Familien wird die Muttersprache ${ }^{289}$ der Haushaltsvorstände herangezogen.

Tabelle 10.4: Muttersprache des Hauhaltsvorstandes

\begin{tabular}{|l|c|cccc|}
\hline Muttersprache & Stichpr. & \multicolumn{4}{|c|}{ Sozioökonomische Schichten } \\
HH-Vorstand & gesamt & A & B & C & D \\
\hline Castellano & $87 \%$ & $98 \%$ & $96 \%$ & $94 \%$ & $72 \%$ \\
\hline Quechua & $12 \%$ & - & $1 \%$ & $5 \%$ & $27 \%$ \\
Aymara & $1 \%$ & - & - & $0 \%$ & $1 \%$ \\
\hline Extranjero & $1 \%$ & $2 \%$ & $3 \%$ & $0 \%$ & - \\
\hline
\end{tabular}

Die meisten Haushaltsvorstände in Lima sind schon mit Castellano aufgewachsen. Wie erwartet, zeigt die Differenzierung nach Schichten deutliche Unterschiede zwischen den Gruppen.

2\% - 3\% der Haushaltsvorstände der beiden oberen Einkommensgruppen sind mit einer ausländischen Sprache aufgewachsen. Hierbei handelt es sich wahrscheinlich um aus dem Ausland eingewanderte Familien. In den beiden unteren Schichten ist dieser Anteil Null. Umgekehrt verhält es sich mit den nativen Sprachen. Während aus der reichsten Schicht kein Haushaltsvorstand mit einer nativen Sprache aufgewachsen ist, beträgt dieser Anteil bei der ärmsten Schicht immerhin 28\%. Hier handelt es sich vornehmlich um vom Land zugereiste Familien.

Die Befragten sollten nach Augenschein außerdem ihrer ethnischen Gruppe zugeordnet werden. Dies ist in Peru nicht einfach, da fast alle Einwohner mittlerweile eine Mischung darstellen. Erwartungsgemäß wurden 97\% als Mestizen bezeichnet. Nur 12\% der obersten Schicht wurden als weiß bezeichnet. Indígenas wurden nur in den beiden unteren Schicht angetroffen $(2 \%, 1 \%)$ und Asiaten nur in den beiden mittleren Schichten (2\%, 0,5\%). ${ }^{290}$

289 Dazu wurden die Haushaltsvorstände gefragt, welche Sprache sie in den ersten Lebensjahren erlernt haben. Dieses Vorgehen wurde gewählt, um von der heute vornehmlich genutzten Sprache zu unterscheiden und Missverständnisse zu vermeiden.

290 Einwanderer aus Nordamerika und Europa scheinen sich vornehmlich in den obersten Schichten der limenser Bevölkerung etabliert zu haben. Bürger asiatischer Abstammung sind immerhin in den Mittelschichten zu finden, während Stadtbewohner indigener Abstammung den Aufstieg aus den unteren Schichten oft nicht in der ersten Generation zu schaffen scheinen. 
Zum Zeitpunkt der Umfrage sprachen nahezu alle limenser Familien vornehmlich Castellano. Die einzigen Ausnahmen bilden 2\% der obersten Schicht, die hauptsächlich eine ausländische Sprache sprechen und 0,3\% der untersten Schicht, die heute noch vornehmlich Quechua sprechen.

Als ein weiterer Hinweis auf die Werte, Normen und Traditionen wurde die geografische Abstammung herangezogen. Deshalb wurde nach dem Migrationsverhalten der Eltern gefragt. Wie sich das Migrationsverhalten in den einzelnen sozioökonomischen Schichten verhält, zeigt die Tabelle.

Tabelle 10.5: Migrationsverhalten der Eltern

\begin{tabular}{|ll|cccc|}
\hline Migrationsverhalten & & \multicolumn{4}{|c|}{ Sozioökonomische Schichten } \\
der Eltern & & A & B & C & D \\
\hline in Lima geboren & Vater & $69 \%$ & $69 \%$ & $47 \%$ & $32 \%$ \\
& Mutter & $79 \%$ & $66 \%$ & $51 \%$ & $27 \%$ \\
\hline \multirow{2}{*}{ aus der Stadt zugezogen } & Vater & $26 \%$ & $20 \%$ & $37 \%$ & $29 \%$ \\
& Mutter & $17 \%$ & $26 \%$ & $29 \%$ & $30 \%$ \\
\hline \multirow{2}{*}{ vom Land zugezogen } & Vater & $5 \%$ & $11 \%$ & $16 \%$ & $39 \%$ \\
& Mutter & $4 \%$ & $8 \%$ & $19 \%$ & $43 \%$ \\
\hline
\end{tabular}

Nur knapp ein Drittel der Eltern von Kindern aus der untersten Schicht ist schon in Lima geboren worden. Der Rest ist erst später zugezogen. Über 40\% kamen vom Land und weitere 30\% kamen aus einer anderen Stadt. In der obersten Schicht dreht sich dieses Verhältnis um. Hier ist nur eine Drittel der Eltern zugezogen, und fast alle kamen aus einer Stadt.

Inwieweit noch heute ein Einfluss der Herkunft der Familien auf ihr städtisches Leben zu erwarten ist, hängt auch mit dem Zeitpunkt ihres Zuzugs zusammen. Die meisten der zugewanderten Väter (75\%) leben schon seit mehr als 20 Jahren in Lima. Dieser Wert liegt für die Mütter etwas niedriger (68\%). Während der Anteil der Väter, die erst in den letzten 2 Jahren zugezogen sind, in keiner Einkommensgruppe besonderes Gewicht erlangt, liegt dieser Anteil bei den Frauen der reichsten Gruppe bei 12\%. Die Tabelle zeigt die durchschnittliche Anzahl an Jahren, die die zugereisten Eltern zum Zeitpunkt der Umfrage bereits in Lima waren.

Tabelle 10.6: Anwesenheit in Lima, Jahre

\begin{tabular}{|l|cccc|}
\hline Migrationsverhalten & \multicolumn{4}{|c|}{ Sozioökonomische Schichten } \\
$\varnothing$ Anzahl Jahre in Lima & A & B & C & D \\
\hline Vater & 32 & 30 & 29 & 27 \\
Mutter & 24 & 25 & 26 & 25 \\
\hline
\end{tabular}

Für die Mütter ergibt sich nach Schichten differenziert kaum ein Unterschied. Die Väter der oberen Schichten sind hingegen im Durchschnitt bereits etwas länger in Lima als die der unteren Schichten. 
Die Eltern aus den verschiedenen sozioökonomischen Gruppen kamen aus ganz unterschiedlichen Motiven nach Lima. Hier interessiert vor allem, ob die besseren Ausbildungsmöglichkeiten in der Hauptstadt eine Rolle gespielt haben.

Tabelle 10.7: Motive für die Migration nach Lima

\begin{tabular}{|l|c|cccc|}
\hline Motive & Stichpr. & \multicolumn{4}{|c|}{ Sozioökonomische Schichten } \\
der Väter & gesamt & A & B & C & D \\
\hline Arbeit & $47 \%$ & $16 \%$ & $12 \%$ & $42 \%$ & $56 \%$ \\
Ausbildung & $27 \%$ & $52 \%$ & $51 \%$ & $35 \%$ & $18 \%$ \\
familiäre Gründe & $25 \%$ & $32 \%$ & $35 \%$ & $22 \%$ & $26 \%$ \\
Terrorismus & $1 \%$ & - & - & $1 \%$ & $1 \%$ \\
\hline \multicolumn{1}{|c|}{ der Mütter } & gesamt & A & B & C & D \\
\hline Arbeit & 31 & $6 \%$ & $12 \%$ & $24 \%$ & $39 \%$ \\
Ausbildung & $20 \%$ & $29 \%$ & $24 \%$ & $30 \%$ & $13 \%$ \\
familiäre Gründe & $49 \%$ & $59 \%$ & $64 \%$ & $46 \%$ & $48 \%$ \\
Terrorismus & $0,1 \%$ & $6 \%$ & - & - & - \\
\hline
\end{tabular}

Tatsächlich stand die Ausbildung als Migrationsgrund bei den Vätern der beiden oberen Schichten an erster Stelle. Mehr als die Hälfte der zugereisten Väter kamen aus diesem Grund. Anders sieht die Verteilung bei den beiden anderen Schichten aus. Aus der untersten Schicht kam mehr als die Hälfte der Väter wegen der Arbeit nach Lima. Nur 18\% kamen wegen der Ausbildung.

Mehr als die Hälfte der Mütter aus den beiden oberen Einkommensgruppen kamen aus familiären Gründen nach Lima. Dennoch stand die Ausbildung bei ihnen an zweiter Stelle. Auch die meisten Mütter aus der untersten Schicht kamen aus familiären Gründen. Der zweithäufigste Grund war allerdings die Arbeit und die Ausbildung nannten nur 13\% als Grund für ihren Zuzug.

Die Migrationsgründe liefern einen direkten Hinweis auf die unterschiedliche Präferenzstruktur zwischen den Schichten. Offensichtlich hat die Ausbildung in den oberen Schichten einen höheren Stellenwert als in den unteren Schichten. Dabei ist zu beachten, dass ein Großteil der zugereisten Eltern der obersten Schichten aus einer anderen Stadt kamen. Dort wird das Bildungsangebot sogar noch besser gewesen sein, als in den Dörfern, aus denen die meisten Eltern der unteren Schichten kamen. Außerdem scheint die Bildung für die Männer wichtiger zu sein als für die Frauen. Dies jedenfalls lassen die Daten für die Generation der Eltern vermuten. 


\subsubsection{Ausbildungssituation der Familien}

Da die empirische Literatur zur Bildungsnachfrage einen deutlichen Zusammenhang zwischen dem Bildungsniveau der Eltern - und vor allem dem Bildungsniveau der Mutter - und der Ausbildung der Kinder nachweist, wird hier das Bildungsniveau beider Elternteile in den verschiedenen Schichten dargestellt. Das Bildungsniveau des Haushaltsvorstandes bildete ein Kriterium zur Einteilung der Schichten. Die folgende Tabelle gibt einen Überblick über die Schulbildung der Eltern.

Tabelle 10.8: Schulbildung der Eltern

\begin{tabular}{|rr|cccc|}
\hline \multicolumn{3}{|l|}{ Schulausbildung der Eltern } & \multicolumn{4}{|c|}{ Sozioökonomische Schichten } \\
& & A & B & C & D \\
\hline \multirow{4}{*}{ Vater } & Keine Schulbildung & - & - & - & $1 \%$ \\
\cline { 2 - 6 } & Primaria & - & - & $9 \%$ & $25 \%$ \\
& Sekundaria & $3 \%$ & $22 \%$ & $49 \%$ & $58 \%$ \\
& Weiterführende Schule & $97 \%$ & $78 \%$ & $42 \%$ & $16 \%$ \\
\hline \multirow{2}{*}{ Mutter } & Keine Schulbildung & - & - & - & $3 \%$ \\
\cline { 2 - 6 } & Primaria & - & - & $11 \%$ & $33 \%$ \\
& Sekundaria & $22 \%$ & $31 \%$ & $52 \%$ & $52 \%$ \\
& Weiterführende Schule & $78 \%$ & $69 \%$ & $37 \%$ & $11 \%$ \\
\hline
\end{tabular}

Zunächst fällt auf, dass fast alle Väter der obersten Schicht nach dem Abschluss der obligatorischen Basisausbildung noch auf eine weiterführende Schule gegangen sind. Von den Müttern sind hier immerhin noch $78 \%$ auf eine höhere Schule gegangen. Fast alle Kinder aus der obersten Schicht werden also von mindestens einem Elternteil mit höherer Bildung betreut.

Von den Eltern der untersten Schicht ist fast ein Drittel nicht über die Primarschule hinaus gekommen. Hinzu kommt, dass von den Eltern, die die Sekundaria begonnen haben, diese oft nach wenigen Jahren wieder verlassen haben. Nur $62 \%$ der Väter der untersten Schicht, die die Sekundarschule besucht haben, haben das 5. Jahr abgeschlossen. Von den Müttern haben sogar nur 24\% das letzte Jahr abgeschlossen. Ebenso hohe Abbrecherraten wurden für die höhere Bildung angegeben.

Tabelle 10.9: Abbruch der weiterführenden Schule nach maximal 3 Jahren

\begin{tabular}{|l|cccc|}
\hline Abbruch der weiterführenden & \multicolumn{4}{|c|}{ Sozioökonomische Schichten } \\
Schule nach max. 3 Jahren & A & B & C & D \\
\hline Vater & $17 \%$ & $18 \%$ & $36 \%$ & $70 \%$ \\
Mutter & $33 \%$ & $41 \%$ & $70 \%$ & $81 \%$ \\
\hline
\end{tabular}

Obwohl immerhin in 16\% der Familien der untersten Schicht mindestens eine Elternteil eine höhere Bildungseinrichtung besucht hat, beschränkt sich diese Erfahrung oft auf nur wenige Jahre. 
Um einen aussagekräftigeren Wert zu erhalten, der einen direkteren Vergleich zwischen den Schichten zulässt, wurden die durchschnittlich abgeschlossenen Schuljahre 291 ermittelt.

Tabelle 10.10: Abgeschlossene Schuljahre der Eltern

\begin{tabular}{|lc|cccc|}
\hline$\varnothing$ abgeschlossene & Stichpr. & \multicolumn{4}{|c|}{ Sozioökonomische Schichten } \\
Schuljahre & gesamt & A & B & C & D \\
\hline Vater & 11,9 & 15,5 & 14,4 & 12,0 & 9,3 \\
Mutter & 11,1 & 14,3 & 13,7 & 11,5 & 8,2 \\
\hline
\end{tabular}

Wie die obige Tabellen erwarten ließen, weisen die Mütter im Schnitt fast ein Jahr Bildung weniger auf als die Väter. Auch der Unterschied zwischen den Schichten ist deutlich zu erkennen. Die Eltern der untersten Schicht haben im Durchschnitt gut sechs Jahre weniger Ausbildung genossen als die Eltern aus der obersten Schicht. Wie die Auswertung der empirischen Studien gezeigt haben, wirkt sich die Ausbildung der Eltern positiv auf die Bildung der Kinder aus. Es ist also zu erwarten, dass die Unterschiede zwischen den Schichten fortgeschrieben werden.

Um über die Ausbildung der Eltern hinaus noch weitere Angaben zum familiären Bildungsniveau zu erhalten, wurde erfragt, ob ältere Geschwister eine höhere Schule besuchen oder besuchten. In 30\% aller Haushalte waren ältere Geschwister vorhanden. In 96\% dieser Haushalte der obersten Schicht und in 55\% der untersten Schicht hat mindestens ein Kind eine weiterführende Schule besucht. Um zu zeigen, ob überhaupt ein Familienmitglied bisher eine höhere Schule besuchte, wurde eine binäre Variable mit diesem Inhalt konstruiert.

Tabelle 10.11: Besuch einer höheren Schule

\begin{tabular}{|l|cccc|}
\hline & \multicolumn{4}{|c|}{ Sozioökonomische Schichten } \\
mind. ein Familienmitglied & A & B & C & D \\
\hline hat eine höhere Schule besucht & $99 \%$ & $93 \%$ & $68 \%$ & $36 \%$ \\
\hline
\end{tabular}

Während in nahezu jeder Familie der obersten Schicht mindestens ein Mitglied eine höhere Schule besuchte, haben nur insgesamt 36\% der Familien der untersten Schicht bisher Erfahrungen mit einer höheren Bildungseinrichtung gemacht.

Außerdem wurde nach den genutzten Informationsmedien gefragt, um einen Indikator über den Informationsstand der Eltern zu erhalten. Das Fernsehen ist mit Abstand das am häufigsten genutzte Informationsmedium. 94\% der limenser Haushalte mit Kindern informieren sich über das Fernsehen über das Welt-

291 Für die Eltern, die eine höhere Bildungsstufe besuchten, wurde angenommen, dass sie die vorausgehende Bildungsstufe abgeschlossen hatten. Dazu wurden für die Primaria 6 Jahre und für die Sekundaria 5 Jahre addiert. In Einzelfällen kann die Anzahl abweichend gewesen sein. Die Unterschiede sollten sich allerdings zwischen den Gruppen ausgleichen. 
geschehen. Die Zeitung liegt mit 51\% auf dem zweiten Platz. Wie häufig die Zeitung in den einzelnen Schichten gelesen wird, zeigt die folgende Tabelle.

Tabelle 10.12: Leseverhalten der Eltern

\begin{tabular}{|l|c|cccc|}
\hline Häufigkeit des & Stichpr. & \multicolumn{4}{|c|}{ Sozioökonomische Schichten } \\
Zeitunglesens & gesamt & A & B & C & D \\
\hline selten oder nie & $39 \%$ & $9 \%$ & $14 \%$ & $35 \%$ & $55 \%$ \\
Wöchentlich & $17 \%$ & $12 \%$ & $22 \%$ & $16 \%$ & $16 \%$ \\
Mehrmals wöchentl. - täglich & $43 \%$ & $79 \%$ & $64 \%$ & $50 \%$ & $29 \%$ \\
\hline
\end{tabular}

Mehr als die Hälfte der Eltern der untersten Schicht lesen die Zeitung nur selten oder nie. Dies sind in der obersten Gruppe nicht einmal 10\%. Ob diese Ergebnisse vor allem aus unterschiedlichen Informationsbedürfnissen oder Verfügbarkeiten resultieren kann hier nicht festgestellt werden. Sicher ist allerdings, dass die Haushalte der oberen Schichten im Schnitt besser informiert sind. Außerdem trainieren sie ihre Lesefähigkeit regelmäßiger. Wenn das Lesen zur täglichen Aktivität der Eltern zählt, ist davon auszugehen, dass auch die Kinder das „Lesen können“ als notwendig oder selbstverständlich ansehen und damit der Lesefähigkeit einen hohen Wert beimessen.

\subsection{4 Ökonomische Situation des Haushaltes}

Zur Einteilung der Haushalte nach Schichten wurde der Beruf des Haushaltsvorstandes, das monatliche Haushaltseinkommen und einige Indikatoren zu den Wohnverhältnissen herangezogen. Die Daten liefern noch ein paar weitere Indikatoren, die die ökonomische Situation der Haushalte beschreiben, auch sie werden unten dargestellt.

Als erstes Kriterium wurde nach dem Beruf der Eltern gefragt ${ }^{292}$. In der obersten Schicht haben $72 \%$ der Väter ihren Beruf mit 'Miembro Poder Ejecutivo, Cuerpo Legislativo' oder 'Profesionales, Científicos e Intelectuales' angegeben. Die 'Profesionales, Científicos e Intelectuales' bilden mit 32\% auch noch in der zweiten Schicht den Berufsschwerpunkt. In der dritten Schicht ist das Berufsfeld weiter aufgefächert. In der untersten Schicht sind 52\% der Väter im Minenbereich und im Bau beschäftigt.

56\% der limenser Mütter bezeichnen sich als Hausfrauen. In der obersten Einkommensschicht gaben weitere $15 \%$ an, 'Pofesionales, Científicos e Intelectuales' zu sein. Diese Berufe üben auch noch 11\% der Mütter der zweiten Schicht aus, in der weitere $14 \%$ im Büro beschäftigt sind. In der dritten und untersten Schicht gaben $19 \%$ bzw. 33\% an 'Trabajadores calificados y no calificados' zu sein.

292 Die Frage wurde offen gestellt und die Antworten dann zu Berufsgruppen zusammengefasst. 
Weitere Daten zur Beschäftigung der Eltern gibt die folgende Tabelle.

Tabelle 10.13: Beschäftigungssituation der Eltern

\begin{tabular}{|l|c|cccc|}
\hline \multicolumn{2}{|l|}{ Beschäftigung der Eltern } & \multicolumn{4}{|c|}{ Sozioökonomische Schichten } \\
\multicolumn{2}{|l|}{} & A & B & C & D \\
\hline haben z. Zt. Arbeit & Väter & $99 \%$ & $95 \%$ & $93 \%$ & $93 \%$ \\
& Mütter & $47 \%$ & $45 \%$ & $35 \%$ & $40 \%$ \\
\hline Arbeit ist dauerhaft & Väter & $99 \%$ & $95 \%$ & $94 \%$ & $80 \%$ \\
& Mütter & $95 \%$ & $91 \%$ & $86 \%$ & $82 \%$ \\
\hline
\end{tabular}

Von den Müttern aller Schichten ist weniger als die Hälfte zur Zeit der Umfrage eine bezahlten Arbeit nachgegangen. In den unteren Schichten sind etwas weniger Frauen berufstätig als in den obern Schichten. Fast alle Männer der obersten Schicht hatten zur Zeit der Umfrage Arbeit und stuften diese auch als dauerhaft ein. Von den Vätern der untersten Schicht waren 7\% arbeitslos und von den arbeitenden Männern stuften 20\% ihre Arbeit als nicht dauerhaft ein. Die Arbeitsmarktsituation ist demnach für die Haushalte der unteren Schichten als deutlich schlechter zu charakterisieren.

Die monatlichen Familieneinkommen ${ }^{293}$ variieren stark zwischen den Schichten. Während in der obersten Schicht keiner Familie weniger als 1.000 Soles monatlich zu Verfügung standen, mussten in der untersten Schicht $75 \%$ der Familien mit dieser Summe auskommen. In der untersten Schicht verdiente kein Haushalt mehr als 4000 Soles im Monat. In der obersten Schicht waren dies immerhin 62\%. Die Tabelle gibt die durchschnittlichen Familieneinkommen an.

Tabelle 10.14: Durchschnittliches monatliches Einkommen

\begin{tabular}{|l|c|cccc|}
\hline$\varnothing$ monatliches Einkommen & Stichpr. & \multicolumn{4}{|c|}{ Sozioökonomische Schichten } \\
Soles & gesamt & A & B & C & D \\
\hline pro Haushalt & 2.086 & 6500 & 3522 & 1252 & 801 \\
pro Kopf & 432 & 1419 & 720 & 263 & 149 \\
\hline
\end{tabular}

Wird das Pro-Kopf-Einkommen betrachtet, wird der Unterschied noch größer. Die unterste Schicht hat dann im Durchschnitt nur gut 10\% des Einkommens der obersten Schicht zur Verfügung.

293 Für jedes Haushaltsmitglied wurde jede Art Einkommen erfragt. Diese Beträge wurde für alle Haushaltsmitglieder addiert und auf monatliche Beträge umgerechnet. 
Die Wohnverhältnisse bieten einen weiteren Indikator für die ökonomische Situation der Haushalte. Die Tabelle stellt die durchschnittlichen Größenverhältnisse des Wohnraums dar.

Tabelle 10.15: Wohnverhältnisse der Familien

\begin{tabular}{|l|c|cccc|}
\hline$\varnothing$ Wohnverhältnisse & Stichpr. & \multicolumn{4}{|c|}{ Sozioökonomische Schichten } \\
Wohnungsgöße & gesamt & A & B & C & D \\
\hline Personenzahl & 5,4 & 4,8 & 5,1 & 5,4 & 5,9 \\
Anzahl Zimmer & 4,5 & 6,8 & 6,1 & 4,1 & 3,4 \\
Personen pro Schlafzimmer & 2,4 & 1,3 & 1,5 & 2,5 & 3,0 \\
\hline
\end{tabular}

Als weiteres Beschreibungsmerkmal wurde die Ausstattung der Wohnungen herangezogen. Die folgende Tabelle zeigt die unterschiedliche Geräteausstattung in den Schichten.

Tabelle 10.16: Geräteausstattung der Wohnungen

\begin{tabular}{|l|cccc|}
\hline Durchschnittliche & \multicolumn{4}{|c|}{ Sozioökonomische Schichten } \\
Anzahl & A & B & C & D \\
\hline Telefonanschluß & 1,7 & 1,2 & 0,7 & 0,3 \\
TV, Farbe & 2,4 & 2,0 & 1,0 & 0,6 \\
Waschmaschine & 1,0 & 0,8 & 0,4 & 0,1 \\
Kühlschrank & 1,2 & 1,1 & 0,9 & 0,6 \\
Auto & 1,7 & 0,7 & 0,2 & 0,1 \\
\hline
\end{tabular}

Während ein beachtlicher Anteil der Haushalte der obersten Schicht über zwei Telefonanschlüsse verfügt, haben ca. 70\% der ärmsten Haushalte gar kein Telefon. Auffällig ist außerdem, dass jeder Haushalt der obersten Schicht über eine eigene Waschmaschine und mindestens ein Auto verfügt, während nur jeder zehnte Haushalt der untersten Schicht diese Dinge zu seinem Eigentum zählt. 


\subsubsection{Zusammenfassung der sozioökonomischen Charakterisierung}

Eine Zusammenfassung der wichtigsten Charakteristika, in denen sich die Haushalte der verschiedenen Schichten unterscheiden, gibt die folgende Tabelle.

Tabelle 10.17: Sozioökonomische Charakteristika der Haushalte

\begin{tabular}{|c|c|c|c|c|}
\hline \multirow{2}{*}{$\begin{array}{l}\text { Durchschnittliche Charakteristika } \\
\text { der Schichten }\end{array}$} & \multicolumn{4}{|c|}{ Sozioökonomische Schichten } \\
\hline & A & $\mathrm{B}$ & C & $\mathrm{D}$ \\
\hline$\varnothing$ Haushaltsgröße & 4,7 & 4,9 & 5,2 & 5,7 \\
\hline Geburtsort des Vater & Lima & Lima & Lima/Stadt & Land/Stadt \\
\hline Migrationsgrund des Vater & Ausbildung & Ausbildung & Arbeit & Arbeit \\
\hline$\varnothing$ Schuljahre des Vater & 16 & 14 & 12 & 9 \\
\hline $\begin{array}{l}\text { Mindestens ein Familienmitglied hat } \\
\text { eine höhere Schule besucht }\end{array}$ & $99 \%$ & $93 \%$ & $68 \%$ & $36 \%$ \\
\hline $\begin{array}{l}\text { Eltern lesen mehrmals wöchentlich } \\
\text { die Zeitung }\end{array}$ & $79 \%$ & $64 \%$ & $50 \%$ & $29 \%$ \\
\hline Vater hat dauerhafte Beschäftigung & $99 \%$ & $95 \%$ & $94 \%$ & $80 \%$ \\
\hline$\varnothing$ monatl. Pro-Kopf-Einkommen & $1.419 \mathrm{~S} . /$ & $720 \mathrm{~S} . /$ & $263 \mathrm{~S} . /$ & 149 S.I \\
\hline$\varnothing$ Personen pro Schlafzimmer & 1,3 & 1,5 & 2,3 & 3,0 \\
\hline$\varnothing$ Anzahl Autos & 1,7 & 0,7 & 0,2 & 0,1 \\
\hline
\end{tabular}

Diese Merkmale können Erklärungshinweise liefern, wenn das Bildungsverhalten der Haushalte analysiert wird. Zunächst bilden sie den Hintergrund für die weiteren Auswertungen, die jeweils nach sozioökonomischen Schichten differenziert werden. 


\subsection{Bildungssituation der Kinder}

In diesem Kapitel wird die Bildungssituation der Kinder der oben beschriebenen Haushalte dargestellt. Dabei soll untersucht werden, ob die Kinder der verschiedenen Schichten sich in ihrem Bildungsverhalten oder ihren Leistungen unterscheiden. Die Befragung der 801 Haushalte lieferte Daten für insgesamt 1.545 Kinder im schulpflichtigen Alter. ${ }^{294}$ Für sie wurden ausführliche Daten zu Beschreibung ihrer Bildungssituation erhoben. Die Daten wurden alle nach sozioökonomischen Schichten getrennt ausgewertet.

\subsubsection{Der typische Bildungsweg}

Um einen typischen Bildungsverlauf nachzuzeichnen, werden zunächst die Arten der besuchten Einrichtungen dargestellt. Außerdem wurden Daten zur Unterstützung des Schulbesuchs erfragt.

Mittlerweile ist allgemein akzeptiert, dass vorschulische Einrichtungen einen wertvollen Beitrag zur Vorbereitung auf die Primarschule leisten. Deshalb wurde zunächst untersucht, wie viele der befragten Kinder einen Kindergarten oder eine Vorschule besucht haben. Die Tabelle fasst die Ergebnisse zusammen.

Tabelle 10.18: Besuch eines Kindergartens

\begin{tabular}{|l|c|cccc|}
\hline Besuch eines & Stichpr. & \multicolumn{4}{|c|}{ Sozioökonomische Schichten } \\
Kindergartens & gesamt & A & B & C & D \\
\hline Gesamt & $77 \%$ & $95 \%$ & $95 \%$ & $80 \%$ & $69 \%$ \\
\hline $6-11$ & $83 \%$ & $99 \%$ & $98 \%$ & $84 \%$ & $78 \%$ \\
$>11$ & $72 \%$ & $91 \%$ & $91 \%$ & $77 \%$ & $62 \%$ \\
\hline
\end{tabular}

Während 95\% der Kinder der beiden oberen Schichten ihre Bildungslaufbahn mit einer vorschulischen Einrichtung begonnen haben, waren dies nur knapp $70 \%$ der untersten Schicht. Allerdings scheint in allen Bevölkerungsschichten die Wertschätzung der bzw. der Zugang zu vorschulischen Einrichtungen mit der Zeit gestiegen zu sein. Nach dem Alter differenziert fältt auf, dass von den jüngeren Schülern bereits mehr einen Kindergarten besucht haben als von den älteren Kindern. Während fast alle Grundschüler der obersten Schichten ihre Bildungskarriere bereits mit einer Vorbereitung begannen, starteten immerhin noch ca. 20\% der beiden unteren Schichten die Grundschule unvorbereitet. Schon vor dem eigentlichen Beginn der Basisausbildung hat ein nicht unerheblicher Teil der Kinder der unteren Schichten einen Nachteil zu verzeichnen.

294 Davon waren 765 zwischen 6 und 11 Jahre alt und damit im Primarschulalter. 780 Kinder waren 12 Jahre und älter. Die Sekundarschule dauert in der Regel 5 Jahre. Kinder die älter als 16 Jahre waren, sind nur dann in die Auswertung integriert worden, wenn sie die Basisausbildung noch nicht abgeschlossen hatten. 
Noch deutlichere Unterschiede zwischen den Schichten zeigen sich bei der Auswahl der Schulen. Familien aus den beiden oberen Schichten wählen vornehmlich private Schulen für ihre Kinder, während Familien der unteren Schichten ihre Kinder vor allem in öffentliche Schulen schicken.

Tabelle 10.19: Art der besuchten Schule

\begin{tabular}{|l|c|cccc|}
\hline Typ des & Stichpr. & \multicolumn{4}{|c|}{ Sozioökonomische Schichten } \\
Bildungszentrums & gesamt & A & B & C & D \\
\hline Öffentlich & $67 \%$ & $7 \%$ & $20 \%$ & $66 \%$ & $81 \%$ \\
Privat & $26 \%$ & $90 \%$ & $72 \%$ & $26 \%$ & $11 \%$ \\
Sonstige & $8 \%$ & $4 \%$ & $8 \%$ & $7 \%$ & $8 \%$ \\
\hline
\end{tabular}

Fast $90 \%$ der Kinder der obersten Einkommensgruppe gehen in eine Privatschule, in der untersten Gruppe sind dies nur noch $11 \%$. Unter den sonstigen Schulen sind vor allem die Parroquiales zu nennen. Dies sind von der Kirche unterstützte Schulen, die vor allem von Kindern ärmerer Familien besucht werden. Die Daten zeigen, dass die Kinder der beiden extremen Schichten kaum die selben Bildungseinrichtungen besuchen. 295

Dies ist kritisch zu betrachten, da in privaten Schulen von einer höheren Qualität der Ausbildung ausgegangen werden muss. Eine Studie der Weltbank über die peruanische Ausbildungssituation kommt zu dem Ergebnis, dass Schüler an privaten Schulen deutlich bessere Ergebnisse erzielen als ihre Mitschüler an staatliche Institutionen. 296

Die Schüler unterschiedlicher sozioökonomischer Gruppen nutzen auch unterschiedliche Transportmittel, um zu ihren Schulen zu gelangen.

Tabelle 10.20: Transportmittel zur Schule

\begin{tabular}{|l|c|rccc|}
\hline Genutztes & $\varnothing$ Zeit- & \multicolumn{4}{|c|}{ Sozioökonomische Schichten } \\
Transportmittel & bedarf & A & B & C & D \\
\hline Öffentlich & 23 Min. & $18 \%$ & $30 \%$ & $29 \%$ & $23 \%$ \\
Privat & 16 Min. & $71 \%$ & $34 \%$ & $6 \%$ & $1 \%$ \\
Zu Fuß & 10 Min. & $10 \%$ & $36 \%$ & $65 \%$ & $75 \%$ \\
\hline$\varnothing$ Zeitbedarf & 14 Min. & 17 Min. & 14 Min. & 14 Min. & 14 Min. \\
\hline
\end{tabular}

Während 71\% der Kinder der obersten Einkommensgruppe ein privates Transportmittel benutzen, sind dies in der untersten Gruppe nur 1\%. Umgekehrt verhält sich die Nutzung der eigenen Beine: In der obersten Einkommensgruppe laufen nur 10\% der Kinder zur Schule, in der untersten Einkommensgruppe sind dies immerhin 75\%. Öffentliche Verkehrsmittel werden relativ ausgeglichen von

295 Unabhängig vom besuchten Schultyp zeigen die Daten, dass die von den Kindern der oberen Schichten besuchten Schulen besser mit sanitären Einrichtungen ausgestattet sind, als die der Kinder der Unteren Schichten.

296 Die Studie untersucht die Einflüsse von zahlreichen Faktoren zur Ausstattung, Lehrercharakteristika, Region usw. auf die durchschnittlichen Ergebnisse von Mathematiktests an den untersuchten Schulen. Die Variable `Schultyp` weist den größten signifikanten Effekt auf. Vgl. Weltbank (2001), S. 108. 
allen Schichten benutzt. 297 Ungeachtet der genutzten Verkehrsmittel benötigen fast $75 \%$ aller Schüler nicht mehr als 15 Minuten für ihren Schulweg. Obwohl die Kinder der oberen Einkommensgruppen vornehmlich zur Schule fahren, brauchen sie im Durchschnitt etwas länger. Da nicht wahrscheinlich ist, dass gerade die 'besseren' Wohngegenden einen Mangel an Schulen aufweisen, lassen diese Zahlen darauf schließen, dass die Eltern der oberen Schichten mehr Aufwand bei der Auswahl der Schulen betreiben und dann auch längere Anfahrtswege - und damit höhere Kosten - in Kauf nehmen.

In Lima können die Kinder alternativ vormittags oder nachmittags den Unterricht besuchen. Die meisten Kinder im schulpflichtigen Alter gehen vormittags zur Schule. Allerdings besucht aus den beiden unteren Schichten auch ein deutlicher Anteil der Schüler den Nachmittagsunterricht.

Tabelle 10.21: Schulturnus

\begin{tabular}{|ll|llll|}
\hline & Schulturnus & \multicolumn{5}{|l|}{ Sozioökonomische Schichten } & \\
& & A & B & C & D \\
\hline 6-11 Jahre & vormittags & $97 \%$ & $88 \%$ & $76 \%$ & $71 \%$ \\
& nachmittags & $3 \%$ & $12 \%$ & $24 \%$ & $29 \%$ \\
\hline 12-18 Jahre & vormittags & $97 \%$ & $90 \%$ & $62 \%$ & $49 \%$ \\
& nachmittags & $3 \%$ & $10 \%$ & $34 \%$ & $49 \%$ \\
& abends & $0 \%$ & $0 \%$ & $3 \%$ & $2 \%$ \\
\hline
\end{tabular}

In der obersten Bevölkerungsschicht besuchen 97\% der Kinder die Schule am Vormittag, die restlichen 3\% gehen am Nachmittag. In dieser Schicht gibt es keinen Unterschied nach dem Alter der Kinder. Diese Relation sinkt mit dem Einkommen. In der untersten Einkommensgruppe besuchen nur noch knapp $60 \%$ der Kinder den Vormittagsunterricht und fast 40\% gehen am Nachmittag. In dieser Schicht zeigen sich große Unterschiede in den Altersgruppen. Während von den Grundschülern nur 30\% den Nachmittagsunterricht besuchen, sind dies bei den älteren Schüler knapp die Hälfte. Von ihnen besuchen auch 2\% eine Abendschule. Ein Grund für den nachmittäglichen Besuch der Schule gerade der älteren Schüler könnte sein, dass sie vormittags im eigenen Haushalt mithelfen 298 oder einer bezahlten Tätigkeit nachgehen müssen. Untersuchungen dazu erfolgen später.

Die Eltern von fast $90 \%$ der Kinder führen mehrmals wöchentlich Gespräche mit ihren Kindern über deren Erfahrungen in der Schule. Der Anteil liegt bei den oberen Einkommensgruppen etwas darüber und bei den unteren Einkommensgruppen etwas darunter. Dementsprechend gaben nur $6 \%$ in der obersten

297 In den oberen Schichten sind dies vor allem Schulbusse, in den unteren Schichten sind dies vor allem Massentransportmittel (combi. microbús, ómnibus).

298 Der Einkauf auf dem Markt und die Zubereitung des Mittagessens sind typische Tätigkeiten die vormittags durchgeführt werden müssen und keinen Aufschub zulassen. 
Gruppe aber $12 \%$ in der untersten Gruppe an, nie oder selten mit ihren Kindern über die Schule zu sprechen.

Tabelle 10.22: Unterstützung des Schulbesuchs durch die Eltern

\begin{tabular}{|l|c|cccc|}
\hline Unterstützung & Stichpr. & \multicolumn{5}{|c|}{ Sozioökonomische Schichten } \\
Gespräche über die Schule & gesamt & A & B & C & D \\
\hline selten oder nie & $11 \%$ & $6 \%$ & $3 \%$ & $12 \%$ & $12 \%$ \\
mehrmals wöchentlich & $89 \%$ & $94 \%$ & $97 \%$ & $88 \%$ & $88 \%$ \\
\hline Hausaufgabenhilfe & gesamt & & & & \\
\hline Nie & $17 \%$ & $13 \%$ & $13 \%$ & $15 \%$ & $20 \%$ \\
\hline bis 5 Stunden pro Woche & $53 \%$ & $55 \%$ & $49 \%$ & $43 \%$ & $60 \%$ \\
mehr als 5 Stunden / Woche & $30 \%$ & $31 \%$ & $38 \%$ & $41 \%$ & $20 \%$ \\
\hline
\end{tabular}

Über die Hälfte der Kinder bekommen in der Woche bis zu 5 Stunden Hausaufgabenunterstützung durch ihre Eltern. Nach sozioökonomischen Schichten differenziert zeigt sich, dass in der untersten Schicht mehr Eltern ihren Kindern keinerlei Unterstützung bei den Hausaufgaben zukommen lassen und weniger Eltern mehr als 5 Stunden helfen als der Durchschnitt für Gesamtlima. In allen anderen Schichten liegt dieses Relation umgekehrt vor. Wenn die Ergebnisse außerdem nach dem Alter der Kinder differenziert werden, zeigt sich, dass vor allem die älteren Kinder weniger Unterstützung bekommen. Während ca. 8\% der 6 bis 11jährigen niemals Unterstützung durch die Eltern erhalten, sind dies bei den Kindern ab 12 Jahre immerhin $27 \%$.

Zusammenfassend lässt sich feststellen, dass die Kinder der oberen Schichten mehr Unterstützung ihres Schulbesuchs durch die Eltern erhalten. Ihnen wird damit eine größere Wertschätzung der Ausbildung durch die Eltern suggeriert. Allerdings ist der Unterschied zwischen den Schichten nicht sehr groß und ein eindeutiges Muster nach Schichten lässt sich auch nicht ableiten.

\subsubsection{Abbrecherverhalten während der Basisausbildung}

Hier soll untersucht werden, ob die Kinder im schulpflichtigen Alter zum Zeitpunkt der Befragung auch tatsächlich zur Schule gingen. Es soll also das Problem des Schulabbruchs untersucht werden. Kinder, die erst verzögert eingeschult werden, werden weiter unten behandelt. Deshalb wurde die Stichprobe auf die 7-18jährigen Kinder begrenzt. Zur Zeit der Befragung besuchten 92\% der befragten Kinder zwischen 7 und 18 Jahren eine Bildungseinrichtung. 
Die Tabelle gibt eine Zusammenfassung der Daten.

Tabelle 10.23: Abbrecherverhalten

\begin{tabular}{|l|r|rrrr|}
\hline $\begin{array}{l}\text { Besuch einer } \\
\text { Bildungseinrichtung }\end{array}$ & $\begin{array}{r}\text { Stichprobe } \\
\text { gesamt }\end{array}$ & \multicolumn{5}{|c}{ A } & B & C & \multicolumn{1}{c|}{ D } \\
\hline Z.Zt. ja & $92 \%$ & $94 \%$ & $95 \%$ & $90 \%$ & $93 \%$ \\
\hline z.Zt. nein & $\mathbf{8 \%}$ & $\mathbf{6 \%}$ & $\mathbf{5 \%}$ & $\mathbf{1 0 \%}$ & $\mathbf{7 \%}$ \\
\hline Sec. Abgeschlossen & $77 \%$ & $100 \%$ & $92 \%$ & $91 \%$ & $52 \%$ \\
\hline Prim. Verlassen & $11 \%$ & $0 \%$ & $0 \%$ & $0 \%$ & $27 \%$ \\
Sec. Abgebrochen & $13 \%$ & $0 \%$ & $8 \%$ & $9 \%$ & $21 \%$ \\
\hline
\end{tabular}

Zunächst ist anzumerken, dass aus den beiden unteren Schichten mehr Schüler zur Zeit der Umfrage schon die Schule verlassen hatten. Allerdings ist der Unterschied gering und es ist kein eindeutiger Trend nach sozioökonomischen Schichten festzustellen. Dieses Bild ändert sich jedoch, wenn die Daten nach erreichten Bildungsniveaus differenziert werden. Aus der obersten Schicht haben alle Schulabgänger den Sekundarabschluss erreicht und damit ihre Ausbildungspflicht erfüllt. Aus der untersten Schicht hat nur die Hälfte der Schulabbrecher den Sekundarabschluss erreicht. 27\% haben die Sekundaria nicht einmal begonnen, sie sind bereits aus oder nach der Primaria abgegangen.

Betrachtet man den Grund für den Rückzug aus dem Schulsystem, bestätigen sich die Unterschiede zwischen den Gruppen.

Tabelle 10.24: Gründe für das Verlassen der Schule

\begin{tabular}{|l|r|rrrr|}
\hline $\begin{array}{l}\text { Grund für das Ver- } \\
\text { lassen der Schule }\end{array}$ & $\begin{array}{r}\text { Stichprobe } \\
\text { gesamt }\end{array}$ & A & B & C & D \\
\hline Niveau erreicht & $77,2 \%$ & $100 \%$ & $100 \%$ & $89 \%$ & $50 \%$ \\
wg. Kosten & $12 \%$ & - & - & $5 \%$ & $25 \%$ \\
wg. Arbeit & $5 \%$ & - & - & $2 \%$ & $10 \%$ \\
Sonstiges & $8 \%$ & - & - & $5 \%$ & $15 \%$ \\
\hline
\end{tabular}

Entsprechend den obigen Ergebnissen gab die Mehrheit als Rückzugsgrund das Erreichen des gewünschten Bildungsniveaus an. Der Sekundarabschluss war offensichtlich für diese Abgänger das gewünschte Ausbildungsziel. Nur in den beiden unteren Einkommensschichten wurden auch weitere Gründe für den Rückzug aus dem Bildungssystem genannt. In der untersten Bevölkerungsschicht ist es nur noch die Hälfte der Schulabbrecher, die ihr angestrebtes Bildungsniveau erreicht hat. Auch hier bestätigen sich also die obigen Ergebnisse. Die Kosten werden als zweit wichtigster Grund für den Rückzug genannt. Dennoch haben nur 10\% der Schulabbrecher dieser Schicht die Schule für eine bezahlte Arbeit verlassen.

Zusammenfassend lässt sich sagen, dass der Abbruch der Basisausbildung in Lima keine große Relevanz zu haben scheint. Fast alle Kinder im schulpflichtigen Alter, die während der Befragung nicht zur Schule gingen, hatten die Se- 
kundaria bereits abgeschlossen. Nur in der untersten Schicht hatte knapp die Hälfte der Abgänger keinen Abschluss vorzuweisen. Das sind ca. 4\% der Kinder der untersten Schicht, die zum Zeitpunkt der Befragung die Schule ohne diesen Abschluss verlassen haben. Dabei spielten zwar die Kosten der Ausbildung eine Rolle, aber die Basisausbildung scheint nicht in direkter Konkurrenz zu einer bezahlten Arbeit zu stehen.

\subsubsection{Leistungen in der Basisausbildung}

Hier soll nun untersucht werden, ob die Schüler der verschiedenen Schichten unterschiedliche Leistungen in der Schule erbringen. Da keine objektiven Bewertungen der Schülerleistungen zur Verfügung standen, wurden Hilfsindikatoren für die Leistungen herangezogen. Dazu wurde zunächst untersucht, ob sich die Kinder in der ihrem Alter entsprechenden Bildungsstufe befinden.

Aus der Gesamtheit der Kinder, die während der Befragung 6 bis 11 Jahre alt waren, gingen nur $84 \%$ tatsächlich in die Primaria. Dieser Prozentsatz resultiert aus der Tatsache, dass die meisten Kinder die Schule erst verspätet beginnen.

Tabelle 10.25: Besuch der Bildungsstufen nach dem Alter, Primarschule

\begin{tabular}{|c|c|c|c|c|c|c|}
\hline \multirow{2}{*}{$\begin{array}{l}\text { Bildungsstufe nach } \\
\text { Altersgruppe }\end{array}$} & & \multirow{2}{*}{$\begin{array}{l}\text { Stichpr. } \\
\text { gesamt }\end{array}$} & \multicolumn{4}{|c|}{ Sozioökonomische Schichten } \\
\hline & & & A & B & C & $\mathrm{D}$ \\
\hline Primarschulalter: 6-11 Jahre & Primaria & $84 \%$ & $72 \%$ & $85 \%$ & $83 \%$ & $86 \%$ \\
\hline \multirow[t]{3}{*}{6 Jahre } & keine. & $12 \%$ & $6 \%$ & $0 \%$ & $18 \%$ & $16 \%$ \\
\hline & Vorschule & $73 \%$ & $88 \%$ & $85 \%$ & $71 \%$ & $63 \%$ \\
\hline & Primaria & $15 \%$ & $6 \%$ & $15 \%$ & $12 \%$ & $21 \%$ \\
\hline \multirow[t]{2}{*}{7 Jahre } & Vorschule & $1 \%$ & $21 \%$ & $13 \%$ & $11 \%$ & $7 \%$ \\
\hline & Primaria & $98 \%$ & $79 \%$ & $87 \%$ & $89 \%$ & $91 \%$ \\
\hline
\end{tabular}

Die Daten zeigen, dass ein deutlich größerer Anteil der 6jährigen Kinder aus der oberen Schicht noch eine Vorschuleinrichtung besuchten als aus der untersten Schicht. Nur 6\% der 6jährigen der obersten Schicht hatten ihre Primarausbildung schon begonnen. Aus der untersten Schicht waren immerhin schon 20\% der 6jährigen in der Grundschule und 16\% ihrer Altersgenossen besuchten noch gar keine Bildungseinrichtung. Aus der obersten Schicht verweilten sogar noch $21 \%$ der 7 jährigen in einer Vorschule.

Die gleiche Untersuchung wurde für die Kinder im Sekundarschulalter durchgeführt. Nur 72\% der 12-16 jährigen Kinder gingen tatsächlich in die Sekundaria. Diese Zahlen sind zum Teil durch den späteren Einstieg in die Grundbildung zu erklären. Auffällig ist allerdings, dass ein größerer Anteil der Kinder der obersten Schicht schon die Sekundaria besucht, obwohl diese doch im Schnitt die Schule später beginnen. 
Die Bildungssituation soll anhand zweier Altersstufen genauer betrachtet werden.

Tabelle 10.26: Besuch der Bildungsstufen nach dem Alter, Sekundaria

\begin{tabular}{|c|c|c|c|c|c|c|}
\hline \multirow{2}{*}{$\begin{array}{l}\text { Bildungsstufe nach } \\
\text { Altersgruppen }\end{array}$} & & \multirow{2}{*}{$\begin{array}{l}\text { Stichpr. } \\
\text { gesamt }\end{array}$} & \multicolumn{4}{|c|}{ Sozioökonomische Schichten } \\
\hline & & & A & B & C & D \\
\hline \multirow{2}{*}{$\begin{array}{l}\text { Sekundarschulalter: } \\
\text { 12-16 Jahre }\end{array}$} & Primaria & $27 \%$ & $21 \%$ & $27 \%$ & $25 \%$ & $31 \%$ \\
\hline & Secundaria & $72 \%$ & $77 \%$ & $73 \%$ & $75 \%$ & $69 \%$ \\
\hline \multirow[t]{2}{*}{12 Jahre } & Primaria & $86 \%$ & $100 \%$ & $95 \%$ & $88 \%$ & $80 \%$ \\
\hline & Secundaria & $13 \%$ & $0 \%$ & $5 \%$ & $12 \%$ & $20 \%$ \\
\hline \multirow[t]{2}{*}{14 Jahre } & Primaria & $13 \%$ & $0 \%$ & $0 \%$ & $14 \%$ & $20 \%$ \\
\hline & Secundaria & $87 \%$ & $100 \%$ & $100 \%$ & $86 \%$ & $80 \%$ \\
\hline
\end{tabular}

Während alle 12jährigen Kinder der obersten Schicht (wegen der verspäteten Einschulung) noch in der Primaria verweilten, waren alle 14jährigen Kinder dieser Schicht bereits in der Sekundaria. Aus der untersten Schicht hingegen befanden sich $20 \%$ der 14jährigen noch immer in der Grundschule, obwohl sie diese im Schnitt früher begonnen haben als ihre Mitschüler aus den oberen Schichten. Von den 16jährigen Schülern waren es immer noch 10\%.

Ein Vergleich der abgeschlossenen Schuljahre zwischen den Schichten macht den Bildungsrückstand der Kinder aus den unteren Schichten noch deutlicher.

Tabelle 10.27: Bildungsstufen nach ausgewählten Altersgruppen

\begin{tabular}{|lr|rrrr|}
\hline Bildungsstufe nach & \multicolumn{5}{|c|}{ Sozioökonomische Schichten } \\
Altersgruppen & A & B & C & D \\
\hline 12 Jahre, & 5. Jahr & $0 \%$ & $18 \%$ & $23 \%$ & $26 \%$ \\
noch in Primaria & 6. Jahr & $88 \%$ & $73 \%$ & $64 \%$ & $47 \%$ \\
\hline 14 Jahre & 5. Jahr & - & - & $20 \%$ & $36 \%$ \\
noch in Primaria & 6. Jahr & - & - & $80 \%$ & $64 \%$ \\
\hline 16 Jahre, & 1.-3. Jahr & $30 \%$ & $36 \%$ & $32 \%$ & $56 \%$ \\
Sekundaria & 4.-5. Jahr & $70 \%$ & $64 \%$ & $68 \%$ & $44 \%$ \\
\hline 18 Jahre, & 3.-4. Jahr & $0 \%$ & $17 \%$ & $17 \%$ & $41 \%$ \\
Noch Sekundaria & 5. (letztes) Jahr & $100 \%$ & $83 \%$ & $83 \%$ & $59 \%$ \\
\hline
\end{tabular}

Von den 12jährigen Kindern, die noch in der Primaria verweilten, befanden sich fast alle Kinder der obersten Schicht bereits im letzten Jahr; während aus der untersten Schicht nicht einmal die Hälfte der Grundschulkinder kurz vor dem Abschluss standen. Dieser Trend setzt sich bei den anderen Altersgruppen fort. Vergleicht man also die Schüler einer Altersklasse, zeigt sich, dass die Schüler aus den unteren Schichten einen größeren zeitlichen Bildungsrückstand aufweisen als ihre Mitschüler aus höheren Schichten. Zusammenfassend lässt sich also sagen, dass Kinder der unteren Schichten die Primarschule im Schnitt eher beginnen als die Kinder der oberen Schichten. Allerdings wird dieser Vorsprung innerhalb der Basisausbildung wieder kompensiert, und sie schließen die Basisausbildung im Schnitt mit höherem Alter ab als die Kinder der oberen Schich- 
ten. Dies lässt auf häufigeres Wiederholen in den unteren Schichten schließen. Im nächsten Punkt wird daher das Wiederholerverhalten ausgewertet.

Wie erwartet, hatten deutlich mehr Kinder aus den unteren Schichten zum Zeitpunkt der Umfrage bereits mindestens ein Schuljahr wiederholt als ihre Mitschüler aus höheren Schichten.

Tabelle 10.28: Wiederholerverhalten der Schüler

\begin{tabular}{|c|c|c|c|c|c|}
\hline \multirow[t]{2}{*}{ Wiederholungen } & \multirow{2}{*}{$\begin{array}{l}\text { Stichpr. } \\
\text { gesamt }\end{array}$} & \multicolumn{4}{|c|}{ Sozioökonomische Schichten } \\
\hline & & A & B & C & D \\
\hline Mind. 1 Jahr & $19 \%$ & $9 \%$ & $6 \%$ & $17 \%$ & $27 \%$ \\
\hline$\varnothing$ Anzahl Wiederholungen (gesamt) & 0,25 & 0,09 & 0,06 & 0,23 & 0,38 \\
\hline$\varnothing$ Anzahl Wiederholungen (Wiederholer) & 1,34 & 1,00 & 1,13 & 1,32 & 1,40 \\
\hline
\end{tabular}

27\% der Schüler aus der untersten Schicht haben Wiederholungserfahrungen gemacht. Aus der obersten Schicht waren dies nur 9\%. Außerdem haben die Wiederholer der obersten Schicht nur jeweils ein einziges Jahr wiederholt, während jeder Wiederholer der untersten Schicht im Schnitt 1,4 Jahre wiederholt hat.

Die meisten Schuljahre werden in der Primaria wiederholt. 72\% der Wiederholungen finden in der Grundschule statt, davon 37\% im ersten Schuljahr.

Tabelle 10.29: Wiederholungen nach Bildungsstufen

\begin{tabular}{|l|c|cccc|}
\hline Wiederholungen & Stichpr. & \multicolumn{4}{|c|}{ Sozioökonomische Schichten } \\
& gesamt & A & B & C & D \\
\hline Primaria & $\mathbf{7 2 \%}$ & $\mathbf{5 8 \%}$ & $\mathbf{6 5 \%}$ & $\mathbf{7 3 \%}$ & $\mathbf{7 2 \%}$ \\
\hline davon 1. Jahr & $37 \%$ & $29 \%$ & $73 \%$ & $33 \%$ & $36 \%$ \\
davon 2. Jahr & $20 \%$ & $14 \%$ & $9 \%$ & $20 \%$ & $22 \%$ \\
\hline Sekundaria & $\mathbf{2 8 \%}$ & $\mathbf{4 2 \%}$ & $\mathbf{3 5 \%}$ & $\mathbf{2 7 \%}$ & $\mathbf{2 8 \%}$ \\
\hline
\end{tabular}

Vor allem in den unteren Schichten beginnt das Schulleben demnach oft mit der negativen Erfahrung einer Klassenwiederholung. Für die Grundschule folgt daher ein etwas differenzierteres Bild. 
Die folgende Tabelle gibt die Wiederholerrate an der Gesamtanzahl der Kinder an.

Tabelle 10.30: Wiederholungen in der Primaria

\begin{tabular}{|l|c|cccc|}
\hline Wiederholungen & Stichpr. & \multicolumn{4}{|c|}{ Sozioökonomische Schichten } \\
Gesamtzahl Kinder & gesamt & A & B & C & D \\
\hline Primaria & $\mathbf{1 7 \%}$ & $\mathbf{5 \%}$ & $\mathbf{4 \%}$ & $\mathbf{1 7 \%}$ & $\mathbf{2 6 \%}$ \\
\hline 1. Klasse & $6 \%$ & $1 \%$ & $3 \%$ & $5 \%$ & $9 \%$ \\
2. & $7 \%$ & $1 \%$ & $0 \%$ & $3 \%$ & $6 \%$ \\
3. & $6 \%$ & $1 \%$ & $0 \%$ & $3 \%$ & $5 \%$ \\
4. & $3 \%$ & $0 \%$ & $0 \%$ & $2 \%$ & $2 \%$ \\
5. & $3 \%$ & $1 \%$ & $0 \%$ & $2 \%$ & $3 \%$ \\
6. & $1 \%$ & $0 \%$ & $0 \%$ & $1 \%$ & $1 \%$ \\
\hline Sekundaria & $\mathbf{7 \%}$ & $\mathbf{4 \%}$ & $\mathbf{2 \%}$ & $\mathbf{6 \%}$ & $\mathbf{1 0 \%}$ \\
\hline
\end{tabular}

Wie schon die Auszählung der Bildungsrückstände erwarten ließ, ist das Wiederholen von Schuljahren vor allem ein Problem der unteren Schichten. In der untersten Schicht starteten fast 10\% aller Kinder ihre schulische Laufbahn mit der Wiederholung der ersten Klasse. 26\% der befragten Kinder dieser Schicht hatten zum Zeitpunkt der Befragung bereits ein Jahr der Primaria wiederholt, wobei 62\% dieser Kinder die Primaria noch gar nicht abgeschlossen hatten. Von den Kindern der obersten Schicht gaben nur 5\% an, überhaupt ein Jahr der Primaria wiederholt zu haben. Hier liegt der Schwerpunkt auch nicht auf dem ersten Jahr. Daraus folgt die Vermutung, dass dieses Ungleichgewicht mit dem Besuch einer vorschulischen Einrichtung dieser Schüler erklärt werden kann Um eine weitere Annäherung an die Leistungen der Schüler zu bekommen, wurden die Eltern gefragt, wie sie die Bildungserfolge ihrer Kinder einschätzen.

Tabelle 10.31: Beurteilungen der Schüler durch die eigenen Eltern

\begin{tabular}{|l|c|cccc|}
\hline Beurteilung der & Stichpr. & \multicolumn{4}{|c|}{ Sozioökonomische Schichten } \\
Leistungen & gesamt & A & B & C & D \\
\hline sehr gut & $12 \%$ & $28 \%$ & $24 \%$ & $19 \%$ & $9 \%$ \\
gut & $48 \%$ & $54 \%$ & $51 \%$ & $50 \%$ & $46 \%$ \\
mittelmäßig & $34 \%$ & $18 \%$ & $23 \%$ & $29 \%$ & $41 \%$ \\
schlecht & $3 \%$ & - & $1 \%$ & $2 \%$ & $4 \%$ \\
sehr schlecht & $0,4 \%$ & - & $0,4 \%$ & $0,4 \%$ & $0,3 \%$ \\
\hline
\end{tabular}

Fast 50\% der Schüler bekamen von den Eltern 'gute' Leistungen in der Schule attestiert. Zwischen den Geschlechtern lassen sich keine deutlichen Unterschiede feststellen.

Nach sozioökonomischen Schichten differenziert fällt auf, dass die Urteile in den unteren Schichten schlechter ausfallen. In der obersten Einkommensschicht wurden insgesamt 28\% der Schüler mit 'sehr gut' bewertet, in der untersten Gruppe waren dies nur noch 9\%. Dementsprechend wurden in der oberen Schicht nur 18\% der Schüler mit 'mittelmäßig' bewertet und in der unteren $41 \%$. 
Da die Eltern die Informationen über die Leistungen ihrer Kinder vor allem aus Zeugnissen entnehmen, können diese Daten als gute Indikatoren für die tatsächlichen schulischen Leistungen der Kinder betrachtet werden. Die Daten zeigen, dass die Kinder der oberen Schichten bessere Leistungen erbringen als ihre Mitschüler aus unteren Schichten. Allerdings ist es auch möglich, dass die Eltern höherer Schichten einfach zufriedener sind mit den Leistungen ihrer Kinder.

\subsubsection{Nebentätigkeiten der Schüler}

Mit diesen Nebentätigkeiten soll untersucht werden, ob sich die Kinder neben der Schule noch mit anderen Dingen außer Lernen und Spielen beschäftigen. Hier sollen im Hinblick auf die Opportunitätskosten des Schulbesuchs vor allem die bezahlte Arbeit und die Mithilfe im eigenen Haushalt betrachtet werden.

Insgesamt nur 4\% der befragten Kinder gingen neben der Schule einer bezahlten Tätigkeit nach. Dies sind von den Kindern der oberen Schichten nur 1\% bis $3 \%$, von den Kindern der untersten Schicht allerdings arbeiten 8\% neben der Schule. Die folgende Tabelle fasst die Daten zusammen.

Tabelle 10.32: Ausübung einer bezahlten Beschäftigung

\begin{tabular}{|c|c|cccc|}
\hline bezahlte Tätigkeit & $\begin{array}{c}\text { Stichprobe } \\
\text { gesamt }\end{array}$ & A & B & C & D \\
\hline gesamt & $4 \%$ & $1 \%$ & $3 \%$ & $1 \%$ & $8 \%$ \\
\hline davon Jungen & $61 \%$ & $100 \%$ & $57 \%$ & $60 \%$ & $61 \%$ \\
davon Mädchen & $39 \%$ & $0 \%$ & $43 \%$ & $40 \%$ & $39 \%$ \\
\hline davon 6-11 Jahre & $14 \%$ & $0 \%$ & $14 \%$ & $0 \%$ & $16 \%$ \\
davon >11 Jahre & $86 \%$ & $100 \%$ & $86 \%$ & $100 \%$ & $84 \%$ \\
\hline
\end{tabular}

Auffällig ist zunächst, dass mehr Jungen als Mädchen eine bezahlte Arbeit ausübten. Außerdem sind die meisten arbeitenden Schüler schon 12 Jahre oder älter. Hier scheinen sich die Annahmen über die Opportunitätskosten und die im 2. Teil empirisch ermittelten Zusammenhänge zu bestätigen. Für die Jungen ist die bezahlte Tätigkeit wichtiger als für die Mädchen und die Opportunitätskosten steigen mit dem Alter. Dennoch zeigen die Untersuchungen, dass nur ein sehr kleiner Teil der Schüler überhaupt einer bezahlten Tätigkeit nachgeht. Ob es auf dem Arbeitsmarkt kein Angebot für derartige Jobs gibt oder die Eltern entschieden haben, dass die Kinder sich vollständig auf die Schule konzentrieren sollen, kann hier noch nicht geklärt werden.

Ein weiteres Beschäftigungsfeld für die Schüler ist im eigenen Haushalt zu finden. $72 \%$ aller Schüler helfen den Eltern neben der Schule noch im Haushalt. Die folgende Tabelle fasst die Antworten zusammen. 
Tabelle 10.33: Hilfe im eigenen Haushalt

\begin{tabular}{|l|r|rrrr|}
\hline Hilfe im Haushalt & Stichprobe & \multicolumn{4}{|c|}{ Sozioökonomische Schichten } \\
Stunden pro Tag & gesamt & A & B & C & D \\
\hline hilft nicht & $27 \%$ & $40 \%$ & $31 \%$ & $32 \%$ & $19 \%$ \\
\hline max. 2 Std. & $61 \%$ & $53 \%$ & $62 \%$ & $56 \%$ & $66 \%$ \\
mehr als 3 Stunden & $11 \%$ & $7 \%$ & $6 \%$ & $11 \%$ & $14 \%$ \\
\hline
\end{tabular}

In den Haushalten der unteren Einkommensschichten helfen mehr Schüler im Haushalt und sie helfen außerdem mehr Stunden am Tag. Während in der obersten Schicht 40\% der Kinder überhaupt nicht im Haushalt helfen, sind dies in der untersten Schicht nur 19\%. Insgesamt helfen 61\% der Kinder max. 2 Stunden bei der Hausarbeit. In der obersten Schicht helfen nur 7\% der Kinder mehr als 3 Stunden, in der untersten Schicht sind dies immerhin 14\%.

Nach Geschlechtern differenziert kann auch hier gezeigt werden, dass mehr Mädchen im Haushalt helfen als Jungen. Insgesamt sind es die Mädchen ab 12 Jahren, die am meisten zur Hausarbeit beitragen. Dies trifft am stärksten in den unteren Einkommensschichten zu. In der untersten Schicht helfen nur 12\% der älteren Mädchen gar nicht im Haushalt aber 25\% helfen mehr als drei Stunden täglich.

Auch hier scheinen sich die Annahmen über die Opportunitätskosten zu bestätigen. Die älteren Mädchen sind für den Haushalt wertvoller als die Jungen und die jüngeren Geschwister. Vor allem in den Haushalten der unteren Schichten müssen die Mädchen neben der Schule zur Hausarbeit beitragen, um die Opportunitätskosten des Schulbesuchs gering zu halten. 


\subsubsection{Zusammenfassung der Bildungssituation}

Die obigen Ausführungen haben gezeigt, dass die Teilnahme an der Basisausbildung nahezu umfassend ist. Ein Schulabbruch, bevor die Schulpflicht erfüllt ist, kommt nur in ganz wenigen Haushalten der unteren Schichten vor. Nahezu alle Kinder werden in die Schule geschickt und verbleiben dort, bis sie die Basisausbildung abgeschlossen haben.

Größere Unterschiede zwischen den Schichten zeigen sich allerdings bei der Art des gewählten Bildungsweges und der Leistungen. Die Tabelle fasst die Bildungsmerkmale zusammen, bei denen die Unterschiede zwischen den Gruppen am deutlichsten auftreten.

Tabelle 10.34: Charakteristika der Basisausbildung der Kinder

\begin{tabular}{|l|cccc|}
\hline durchschnittliche Charakteristika & \multicolumn{4}{|c|}{ Sozioökonomische Schichten } \\
nach Schichten & A & B & C & D \\
\hline Besuch eines Kindergartens & $95 \%$ & $95 \%$ & $80 \%$ & $69 \%$ \\
\hline Art der besuchten Schule & privat & privat & öffentlich & öffentlich \\
\hline mind. 1 Jahr wiederholt & $9 \%$ & $6 \%$ & $17 \%$ & $27 \%$ \\
\hline$\varnothing$ Anzahl Wiederholungen & 1 & 1,13 & 1,32 & 1,4 \\
\hline Beurteilung durch die Eltern: sehr gut & $28 \%$ & $24 \%$ & $19 \%$ & $9 \%$ \\
\hline
\end{tabular}

Darüber hinaus erhalten die Kinder der oberen Schichten mehr Unterstützung ihres Schulbesuchs durch die Eltern. Offensichtlich bekommen sie diese Unterstützung nicht, weil sie schlechtere Leistungen erbringen. Ob die besseren Leistungen direkte Folge dieser Unterstützungsmaßnahmen sind, oder ob sie einfach eine höhere Wertschätzung der Eltern für die Schule ausdrücken, und damit die Leistungen über die Motivation der Schüler beeinflussen, kann nicht eindeutig geklärt werden.

Die Kinder der unteren Schichten weisen einen deutlichen zeitlichen Bildungsrückstand gegenüber ihren Mitschülern aus den oberen Schichten auf, obwohl jene ihre Ausbildung häufiger mit einer vorschulischen Einrichtung beginnen und deshalb häufiger später eingeschult werden. Der durchschnittlich frühere Einstieg der Kinder der unteren Schichten in die Basisausbildung wird durch vermehrtes Wiederholen von Schuljahren überkompensiert. Ihre Basisausbildung dauert damit im Schnitt länger.

Bildungsunterschiede zwischen den Schichten zeigen sich also nicht an der Teilnahme an der Basisausbildung, sondern in der Auswahl der Schule und der Leistungen der Schüler. Die Beteiligung an der Basisausbildung ist in Lima über alle Schichten hinweg als umfassend zu bezeichnen. 


\section{Bildungswunsch und Einkommenserwartungen der Eltern}

In diesem Kapitel sollen Erklärungsansätze für die umfassende Nachfrage nach Basisausbildung gefunden werden. Dafür wurden die Haushalte nach einigen Wünschen und Erwartungen bezüglich des Schulbesuchs ihrer Kinder gefragt. Die Beantwortung der Fragen erfolgte in den meisten Fällen durch die Mütter der Kinder. In den wenigen Fällen, in denen der Vater oder ein anderes erwachsenes Mitglied des Haushaltes die Antworten gab, hatte diese Person auch angegeben, die Bildungsentscheidungen für die Kinder (mit) zu treffen.

Insbesondere die Ertragserwartungen bilden den Schwerpunkt dieses Kapitels, da sie nach der Theorie den größten Einflussfaktor für die Bildungsnachfrage darstellen und dennoch in empirischen Untersuchungen bisher vernachlässigt wurden. Hier wird also versucht, die Relevanz der ökonomischen Annahmen zu untersuchen und die Präferenzen der Individuen aufzudecken.

\subsection{Gewünschtes Bildungsniveau für die Kinder}

Um einen Indikator für die elterliche Bildungsnachfrage zu erhalten, wurden die Eltern für jedes einzelne Kind ${ }^{299}$ gefragt, welches Bildungsniveau sie sich für das jeweilige Kind wünschen. Die Ergebnisse sind in der Tabelle zusammengefasst.

Tabelle 11.1: Gewünschtes Bildungsniveau für die eigenen Kinder

\begin{tabular}{|l|r|rrrrr|}
\hline Gewünschtes & Stichprobe & \multicolumn{5}{|c|}{ Sozioökonomische Schichten } \\
Bildungsniveau & gesamt & A & B & C & \multicolumn{1}{c|}{ D } \\
\hline Primaria & $0 \%$ & $1 \%$ & - & $0 \%$ & $1 \%$ \\
Sekundaria & $7 \%$ & - & $2 \%$ & $5 \%$ & $13 \%$ \\
Superior & $92 \%$ & $99 \%$ & $98 \%$ & $94 \%$ & $87 \%$ \\
\hline
\end{tabular}

Für fast alle Kinder der Stichprobe wünschten sich die Eltern das Erreichen einer höheren Bildungsstufe. Nur in der untersten Schicht, könnten 13\% der Schüler ihre Eltern mit dem Abschluss der Basisausbildung befriedigen. Nach dem Geschlecht und dem Alter der Schüler konnten nahezu keine Unterschiede festgestellt werden. Der Bildungswunsch ist nicht nur über die Bevölkerungsschichten, sondern auch über die persönlichen Charakteristika der Kinder hinweg als umfassend zu bezeichnen.

Der umfassende Bildungswunsch erstaunt etwas, da die Zusammenfassung der empirischen Literatur in Kapitel 5 deutlich zum Ausdruck brachte, dass sowohl das Einkommensniveau als auch die Ausbildung der Eltern die Schulbildung der Kinder positiv beeinflussen. Die befragten Bevölkerungsschichten unterschei- 
den sich in beiden Kriterien deutlich voneinander, was einen deutlicheren Unterschied in den Bildungswünschen vermuten ließe. Allerdings zeigen die Daten nicht, wie viel höhere Bildung sich die Eltern für ihre Kinder wünschen. Ein deutlicherer Unterschied kann durchaus erst bei höheren Niveaus zum Ausdruck kommen.

Wie in Kapitel 4 gezeigt wurde, kann davon ausgegangen werden, dass die Bildungsentscheidung zumindest teilweise auf einem rationalen Kalkül basiert. Auch die Zusammenfassung der empirischen Untersuchungen lassen darauf schließen, dass die Nutzen- und Kostenfaktoren einen nicht unerheblichen Einfluss auf die Entscheidung haben. Wird der von den Eltern genannte Ausbildungswunsch als ihre potenzielle Bildungsnachfrage für die Kinder interpretiert, und wird ihnen ein rationales bildungsökonomisches Kalkül unterstellt, können nach der dargestellten Theorie Rückschlüsse auf einige die Bildungsnachfrage beeinflussende Kennzahlen gezogen werden.

Zunächst wird die Ausbildung über die Schulpflicht hinaus offensichtlich als rentabel angenommen. Das heißt, dass die Ertragsrate positiv sein muss und demnach die Nutzen der Ausbildung ihre Kosten übersteigen müssen. ${ }^{300}$ Darüber hinaus muss die Ertragsrate für höhere Bildung mindestens so hoch sein wie der Finanzierungszinssatz oder die Ertragsrate einer alternativen Investition. 301

Während diese Zusammenhänge für fast alle Familien der obersten Schicht zu gelten scheinen, ist der optimale Investitionspunkt für immerhin 13\% der Schüler der untersten Schicht bei der Sekundarbildung erreicht.

Ob die umfassende Bildungsnachfrage tatsächlich mit der erwarteten Rentabilität der Ausbildung erklärt werden kann, zeigen die folgenden Abschnitte.

300 Streng genommen müssen die abdiskontierten Nutzen mindestens den aufdiskontierten Kosten zu einem bestimmten Zeitpunkt entsprechen. Vgl. dazu die Berechnung von Ertragsraten in Anschnitt 2.1.3.

301 Vgl. dazu die Darstellung zur Ermittlung des optimalen Bildungsniveaus in Abschnitt 3.1. 


\subsection{Einkommenserwartungen am Arbeitsmarkt}

In einem ersten Schritt sollte untersucht werden, ob der Ausbildung wirklich eine das Einkommen steigernde Wirkung zugetraut wird. Dazu wurden die Eltern gefragt, wie hoch sie die Einkommen von Arbeitnehmern mit unterschiedlichen Bildungsniveaus einschätzen. Die Tabelle fasst die Antworten zu durchschnittlich genannten Beträgen zusammen.

Tabelle 11.2: Erwartete Einkommensdifferenzen nach Bildungsniveaus, Soles

\begin{tabular}{l|rrrr}
\hline Monatl. Einkommen einer Person & \multicolumn{4}{|c}{ Bevölkerungsschicht } \\
(Durchschnittswerte in Soles) & A & B & C & D \\
\hline ohne Ausbildung & 301 & 317 & 284 & 268 \\
mit Primaria & 421 & 415 & 395 & 345 \\
mit Secundaria & 689 & 656 & 575 & 464 \\
mit höherer Bildung & 2.920 & 1.894 & 1.415 & 960 \\
\hline
\end{tabular}

Die Tabelle zeigt, dass tatsächlich mit steigendem Bildungsniveau höhere Einkommen erwartet werden. Und zwar werden Einkommenssteigerungen unabhängig von der Zugehörigkeit zu einer sozioökonomischen Schicht erwartet. Allerdings fällt auf, dass mit höherem sozioökonomischen Niveau auch höhere Steigerungsraten durch die Ausbildung erwartet werden.

Tabelle 11.3: Erwartete Einkommensdifferenzen nach Bildungsniveaus, \%

\begin{tabular}{l|rrrr}
\hline \% Steigerung von einem & \multicolumn{4}{|c}{ Bevölkerungsschicht } \\
Bildungsniveau zum nächsten & A & B & C & \multicolumn{1}{c}{ D } \\
\hline ohne Ausbildung => Primaria & $+40 \%$ & $+31 \%$ & $+39 \%$ & $+29 \%$ \\
Primaria => Secundaria & $+64 \%$ & $+58 \%$ & $+46 \%$ & $+34 \%$ \\
Secundaria => höherer Bildung & $+324 \%$ & $+189 \%$ & $+146 \%$ & $+107 \%$ \\
\hline
\end{tabular}

Während die Einkommen für Arbeiter ohne Ausbildung noch recht einheitlich niedrig angenommen werden, klaffen die Schätzungen bei steigenden Ausbildungsniveaus immer weiter auseinander. Die Eltern der untersten Schicht glaubten im Durchschnitt, dass eine Person mit höherer Bildung am Arbeitsmarkt monatlich knapp 1.000 Soles verdient. Die Eltern der obersten Schicht hingegen erwarteten für höher ausgebildete Arbeitnehmer ein monatliches Einkommen von fast 3.000 Soles.

Sähen sich alle Haushalte dem gleichen Bildungsangebot und den gleichen Kosten gegenüber, würden die individuell erwarteten Ertragsratenfunktionen der unterschiedlichen Schichten zwar auf einem ähnlichen Niveau beginnen, die Funktionen würden aber für die Haushalte der oberen Schichten immer oberhalb der Funktionen der unteren Schichten liegen und mit steigendem Bildungsniveau immer weiter auseinander klaffen. Bei gleichen Finanzierungsbedingungen lägen dann die optimalen Investitionspunkte der oberen Schichten 
bei höheren Bildungsniveaus als die Investitionspunkte der unteren Schichten. ${ }^{302}$

Außerdem werden die höchsten Einkommenssteigerungen durch den Schritt von der Sekundaria in die höhere Bildung erwartet. Während die Primaria trotz des geringen Ausgangsniveaus nur einen Zuwachs zwischen 30\% und 40\% verspricht, erwarten die Haushalte der obersten Schicht eine Einkommensdifferenz von mehr als 300\% zwischen Absolventen der Sekundaria und einer höheren Schule. Die Haushalte der untersten Schicht, erwarten im Schnitt eine Differenz von $100 \%$. Diese Daten geben einen Hinweis darauf, warum fast alle Haushalte ihre Kinder nach Abschluss der Sekundaria noch auf weiterführende Schulen schicken wollen.

Die Daten zeigen deutlich, dass der größte Einkommensanstieg erst durch den Besuch einer höheren Bildungseinrichtung nach der Basisausbildung erwartet wird. Außerdem wird mit steigendem sozioökonomischen Niveau der Ausbildung eine viel größere, das Einkommen steigernde Wirkung zugetraut. Die Wertschätzung der Ausbildung als Investition in die Zukunft dürfte damit in den oberen Schichten stärker ausgeprägt sein.

Allerdings zeigte die Beschreibung der sozioökonomischen Schichten, dass 90\% der Kinder aus der obersten Schichte eine Privatschule und 81\% der Kinder der untersten Schicht eine öffentliche Schule besuchten. Es ist also nicht davon auszugehen, dass sich die Familien den gleichen Kosten der Ausbildung gegenüber sehen. Weiterhin ist davon auszugehen, dass die Kostenbelastung für die Familien in höheren Bildungsstufen höher ist als in der obligatorischen Basisausbildung. Vor allem weil angenommen werden muss, dass die Opportunitätskosten mit dem Alter und Ausbildungsniveau der Kinder steigen. Die Einkommenserwartungen sagen daher noch nichts über die Rentabilitätserwartungen der Ausbildung aus. Die sollen im folgenden Abschnitt untersucht werden.

302 In der grafischen Darstellung in Kapitel 3 (Grafik 3.2) entspräche dies den Punkten $P_{11} / P_{31}$ bzw. $\mathrm{P}_{13} / \mathrm{P}_{33}$ für zwei alternative Finanzierungskostenkurven. 


\subsection{Das Rentabilitätseinkommen für die eigenen Kinder}

Die Auswertung der empirischen Literatur hat gezeigt, dass es kaum möglich ist, die direkten Kostenfaktoren der Ausbildung zu beobachten. Da es sich hauptsächlich um Opportunitätskosten handelt303, sind diese auch für die Eltern schlecht zu beziffern. Bei der Bildungsentscheidung wird daher eher die vage Idee der Eltern über die Kostenbelastung eine Rolle spielen.

Um dennoch die Erwartungen der Eltern über die Rentabilität der Ausbildung abschätzen zu können, wurden sie direkt danach gefragt. Dazu sollten sie angeben, welches Einkommen sie für ihre Kinder für möglich hielten, wenn diese nach der Primaria bzw. der Sekundaria die Schule verließen. Jeweils direkt im Anschluss sollten sie den Betrag nennen, der ihrer Meinung nach notwendig wäre, damit sich die Investition in die jeweilige Ausbildung gelohnt hätte. Diese Fragestellung wurde gewählt, um einen Indikator für den empfundenen Wert der Ausbildung zu erhalten. Ableitungen über die exakte, tatsächliche Wirtschaftlichkeit der Ausbildung sind damit nicht möglich. Die genannten Beträge werden im nächsten Abschnitt zur ökonomischen Beurteilung der Bildungsstufen durch die Eltern herangezogen. Hier soll zunächst die ökonomische Bedeutung der Rentabilitätseinkommen geklärt werden.

Das Rentabilitätseinkommen wird als das Einkommen verstanden, bei dem die Familien die in die Ausbildung der Kinder investierten Beträge gerade zurückerhalten. Bezogen auf den Investitionsansatz, handelt es sich dabei um das Einkommen, das die Kinder erhalten müssten, damit bei gegebenen Kosten und gegebenem Zins die Investition ausgeglichen wird. Die aufgezinsten Kosten müssen also genau den abgezinsten Nutzen entsprechen. Mit der in Kapitel 2 dargestellten Formel kann also auch das Rentabilitätseinkommen berechnet werden.

$$
\sum_{t=-i}^{0}(\text { Kosten der Ausbildung })_{t}(1+r)^{-t}-\sum_{t=i}^{n}(\text { Nutzen der Ausbildung })_{t}(1+r)^{-t}=0
$$

Würden in diese Gleichung die tatsächlichen Kosten und die erwarteten Einkommen eingesetzt könnte die subjektiv erwartete Ertragsrate der Bildung berechnet werden. ${ }^{304}$ Ebenso kann aber bei gegebenen Kosten und gegebenem Vergleichszins das Einkommen berechnet werden, bei dem der Nettogegenwartswert der Investition Null wird. 305 Dieses Vorgehen sollte hier angewendet werden.

\footnotetext{
303 Vgl. dazu die zusammenfassende Darstellung über die Kostenfaktoren in Kapitel 5.

304 Vgl. dazu das Berechnungsverfahren von Ertragsraten in Kapitel 2.1.

305 Vgl. dazu auch Psacharopoulos (1996), S. 341.
} 
Wenn die Eltern nun unterschiedliche Rentabilitätseinkommen für die gleichen Bildungsniveaus angeben, kann das einerseits daran liegen, dass sie unterschiedliche Kosten für die Ausbildung ihrer Kinder aufbringen müssen. Andererseits können sie auch einen anderen Zins als relevant für die Investition betrachten. 306

Bei den genannten Zahlen handelt es sich natürlich nicht um derart sorgfältig berechnete Werte, sondern vielmehr um die spontane Idee der befragten Eltern. Weil nicht davon ausgegangen werden kann, dass die Eltern aufwendige Kalkulationen durchgeführt haben, bevor sie ihre Kinder in die Schule schickten, werden sie auch keine exakten Werte für die oben erwähnten Einflussfaktoren parat haben.

Wird deshalb realistischerweise davon ausgegangen, dass die Eltern bei den Überlegungen zu ihren Antworten die Verzinsung der Kosten- und Nutzenströme vernachlässigt haben, vereinfacht sich die obige Gleichung zu einer Identität der summierten Kosten und Nutzen. Die genannten Rentabilitätseinkommen können dann als Indikator für die Kosten der Ausbildung betrachtet werden. Da es sich bei den Rentabilitätseinkommen um monatliche Einkommen handelt, kann von diesen Werten nicht direkt auf die Kosten der Ausbildung geschlossen werden. Vielmehr handelt es sich um das monatliche Einkommen, das in der Summe die Investitionskosten ausgleicht. Unterschiedlich angegebene Rentabilitätseinkommen weisen dann auf unterschiedliche Kostenbelastungen der Eltern hin. 307

Wenn nun die Rentabilitätseinkommen mit den erwarteten Einkommen verglichen werden, können wieder Rückschlüsse auf die Ertragsraten der Ausbildung gezogen werden. Liegt das erwartete Einkommen unter dem, das bei gegebenem Vergleichszins den Nettogegenwartswert Null setzt, muss die erwartete Ertragsrate der Bildungsinvestition unter dem Vergleichszins liegen.

Sowohl für die Primaria als auch für die Sekundaria wurden die Rentabilitätseinkommen erfragt. Bei beiden Bildungsstufen handelt es sich um die notwendigen monatlichen Einkommen, um die jeweiligen Gesamtinvestitionen auszugleichen. Die gesamte Ausbildung wird also jeweils gegen die Situation ohne Ausbildung abgewägt. Den Überlegungen liegen also Durchschnittskalküle

306 Als Zins könnte z. B der Marktzins herangezogen werden, zu dem die Investitionskosten finanziert werden müssen. Als weitere Unbekannte könnte die Dauer der Ausbildung und damit die Dauer der Berufstätigkeit betrachtet werden. Obwohl oben gezeigt wurde, dass die Kinder armer Familien häufiger wiederholen als die Kinder reicher Familien, sollen diese Unterschiede in den Kosten- und Nutzenperioden zunächst vernachlässigt werden.

307 Die Aussagen behalten auch Gültigkeit, wenn alle Eltern den gleichen Zins für die Ausbildung als rentabel annehmen würden. Die Annahme vernachlässigter oder identischer Zinsen wird später aufgehoben. 
zugrunde. Wenn für die Sekundaria höhere Rentabilitätseinkommen angegeben werden, bedeutet das, dass durch die Fortführung des Schulbesuchs auch weitere Kosten entstanden sind. 308

Wie in Kapitel 2 dargestellt wurde, wird in der bildungsökonomischen Literatur üblicherweise das Marginalkalkül unterstellt. ${ }^{309}$ Dafür müssen in diesem Fall die Differenzen zwischen beiden Bildungsstufen ermittelt werden.

Die Darstellung der Befragungsergebnisse und eine Interpretation der Daten erfolgt in den folgenden Abschnitten.

308 Die höheren Angaben für die Sekundaria erklären sich zum einen daraus, dass weitere Jahre in die Ausbildung investiert wird und dadurch zusätzliche Kosten entstehen. Zum anderen verkürzt sich aber auch - bei gegebener Lebensdauer - der Verdienstzeitraum.

309 Vgl. dazu die Darstellung der Ertragsratenarten in Kapitel 2.1. 


\subsection{Erwartungen über die Rentabilität der Ausbildung}

Zunächst werden die Ertragserwartungen der Eltern allgemein untersucht, ohne auf Unterschiede zwischen den Bevölkerungsschichten einzugehen. Diese Vorgehen ist wichtig, um Erwartungsmuster aufzudecken, die für alle Schichten ähnlich verlaufen. Diese allgemeinen Erwartungen lassen Schlüsse über den Prozess der Nachfragebildung zu, und erlauben eine kritische Auseinandersetzung mit den, in der bildungsökonomischen Theorie üblichen, Annahmen. Anschließend werden die Unterschiede in den Ertragserwartungen zwischen den Schichten untersucht. Vorhandene Erwartungsunterschiede können herangezogen werden, um Unterschiede im Bildungsverhalten zu erklären.

\subsubsection{Gemeinsamkeiten in den Rentabilitätserwartungen}

In diesem Abschnitt werden die Rentabilitätserwartungen für die Primaria und die Sekundaria untersucht, die für die Kinder aller sozioökonomischen Schichten gleichermaßen gelten. Dazu werden die Befragungsergebnisse für beide Bildungsstufen dargestellt und im Hinblick auf die Bildungsnachfrage interpretiert.

\subsubsection{Gesamtrentabilität der Bildungsstufen}

Wie oben beschrieben, wurden die Eltern nach ihrer Einschätzung über die Rentabilität der Ausbildung ihrer Kinder befragt. Die genannten Beträge für die Einkommenserwartungen und die Rentabilitätseinkommen sind in der folgenden Tabelle zu Durchschnittswerten zusammengefasst. 310

Tabelle 11.4: Einkommens- und Rentabilitätserwartungen der eigenen Kinder

\begin{tabular}{l|l|rrrr}
\hline \multicolumn{2}{l|}{$\begin{array}{l}\text { Monatl. Einkommen einer Person ... } \\
\text { (Durchschnittswerte in Soles) }\end{array}$} & A & B & C & D \\
\hline erwartete Einkommen & Primaria & 442 & 376 & 317 & 327 \\
& Sekundaria & 798 & 713 & 579 & 458 \\
\hline Rentabilitätseinkommen & Primaria & 1.059 & 810 & 659 & 548 \\
& Sekundaria & 1.447 & 1.160 & 948 & 728 \\
\hline Differenz nach & Primaria & -617 & -434 & -342 & -221 \\
& Secundaria & -649 & -447 & -369 & -270 \\
\hline
\end{tabular}

Die Daten zeigen deutlich, dass die durchschnittlich erwarteten Rentabilitätseinkommen für beide Bildungsstufen in jeder Schicht deutlich über den geschätzten möglichen Einkommen der Kinder liegen. Die durchschnittlichen Haushalte jeder Schicht erwarten also hohe absolute Verluste sowohl nach der Primaria

310 Ein Vergleich mit der Tabelle für den Arbeitsmarkt allgemein zeigt, dass die Eltern die Einkommen ihrer eigenen Kinder etwas anders einschätzen als die Einkommensstruktur am Arbeitsmarkt allgemein. Diese Abweichungen werden weiter unten untersucht. 
als auch nach der Sekundaria. Allerdings fällt auch auf, dass nach Abschluss der Sekundaria die Gesamtverluste nicht viel höher erwartet werden als nach der Primaria. Die deutet darauf hin, dass die durch die Sekundaria zusätzlich erwarteten Kosten fast durch die erwarteten Einkommenszuwächse ausgeglichen werden.

Im folgenden soll ein relativer Wert für die Verlusterwartungen ermittelt werden. Um die Gesamtrentabilität der Bildungsstufen zu vergleichen, wird eine Art Deckungsbeitrag ermittelt. Dazu wird berechnet, welchen Anteil die erwarteten monatlichen Einkommen an den genannten monatlichen Rentabilitätseinkommen haben.

Tabelle 11.5: Erwartete Einkommen als \% der Rentabilitätseinkommen

\begin{tabular}{l|c|cccc}
\hline Erwartete Einkommen als \% der & Stichpr. & \multicolumn{4}{|c}{ Bevölkerungsschicht } \\
Remtabilitätseinkommen & gesamt & A & B & C & D \\
\hline Eink. Prim./Rentabilitätseinkommen & $50 \%$ & $42 \%$ & $46 \%$ & $48 \%$ & $60 \%$ \\
Eink. Sec./Rentabilitätseinkommen & $61 \%$ & $55 \%$ & $61 \%$ & $61 \%$ & $63 \%$ \\
\hline
\end{tabular}

Die Tabelle zeigt, dass die Sekundaria in der Gesamtbetrachtung eine höhere Deckungsrate aufweist als die Primaria. Während die Familien glauben, dass die Primaria nur zu 50\% der als notwendig erachteten Einkommen führt, wird nach der Sekundaria schon ein Deckungsanteil von 61\% erwartet.

In allen Schichten werden demnach durchschnittlich größere Einkommens- als Kostenzuwächse durch den Besuch der Sekundaria erwartet. Dennoch bleiben auch nach der Sekundaria noch hohe absolute Verluste in der Einschätzung der Befragten zu verzeichnen.

Um einen Überblick über die Verteilung dieser Verlusterwartungen in den einzelnen Familien zu erhalten, wurde für jeden Haushalt die Differenz zwischen dem erwarteten und dem Rentabilitätseinkommen gebildet. Die folgende Tabelle fasst das Ergebnis zusammen.

Tabelle 11.6: Rentabilitätserwartungen der Ausbildung, eigene Kinder

\begin{tabular}{|c|c|c|c|c|c|}
\hline \multirow{2}{*}{\multicolumn{2}{|c|}{$\begin{array}{l}\text { Differenz zw. erw. Eink. und Rentabilitätseink. } \\
\text { (\% der befraqten Haushalte) }\end{array}$}} & \multicolumn{4}{|c|}{ Bevölkerungsschicht } \\
\hline & & A & B & C & D \\
\hline \multirow{3}{*}{ a) für Primaria } & Rentabel $(>0)$ & $2 \%$ & $0 \%$ & $2 \%$ & $1 \%$ \\
\hline & Indifferent $(=0)$ & $20 \%$ & $13 \%$ & $10 \%$ & $13 \%$ \\
\hline & Nicht rentabel $(<0)$ & $78 \%$ & $86 \%$ & $88 \%$ & $86 \%$ \\
\hline \multirow[t]{3}{*}{ b) für Secundaria } & Rentabel $(>0)$ & $4 \%$ & $4 \%$ & $4 \%$ & $2 \%$ \\
\hline & Indifferent $(=0)$ & $15 \%$ & $13 \%$ & $11 \%$ & $13 \%$ \\
\hline & Nicht rentabel $(<0)$ & $82 \%$ & $84 \%$ & $85 \%$ & $85 \%$ \\
\hline
\end{tabular}

Diese Angaben können als individuelle, ökonomische Wertschätzung der Ausbildung betrachtet werden. Insgesamt halten nur 1,5\% der Haushalte die Primaria und nur 3\% die Sekundarbildung für überhaupt rentabel. Für über $80 \%$ der Befragten liegen die erwarteten Einkommen weit unter denen, die für eine ren- 
table Investition als notwendig empfunden wurden. Die Daten zeigen deutlich, warum sich kaum ein Elternpaar mit der Basisausbildung für ihre Kinder zufrieden geben möchte, wenn diese die Ausbildung erst einmal begonnen haben.

Die kritische Hinterfragung der ökonomischen Modelle in Kapitel 4 hat gezeigt, dass auch Gründe für die Ausbildungsentscheidung verantwortlich sein können, die nicht in der ökonomischen Theorie abgebildet werden können. Ein möglicher Grund, dass die Ausbildung unabhängig von der wirtschaftlichen Rentabilität als notwenig erachtet wird, wurde explizit erfragt. Wenn z.B. die Möglichkeit, ein zum Leben ausreichendes Einkommen zu erwirtschaften, gering ausgebildeten Menschen grundsätzlich verwehrt bleibt, besteht keine Alternative zur Ausbildung. Die Rentabilitätsüberlegungen treten dann in den Hintergrund.

Tabelle 11.7: Reicht die Basisausbildung aus, um den Lebensunterhalt zu sichern?

\begin{tabular}{l|rrrr}
\hline Zur Erzielung eines akzeptablen Einkommens... & \multicolumn{4}{|c}{ Bevölkerungsschicht } \\
(\% der befragten Haushalte) & A & B & C & D \\
\hline ...reicht die Primaria aus. & $1 \%$ & $2 \%$ & $2 \%$ & $3 \%$ \\
...reicht die Sekundaria aus. & $5 \%$ & $2 \%$ & $6 \%$ & $6 \%$ \\
\hline Ausgeb. Bewerber werden immer bevorzugt. & $98 \%$ & $98 \%$ & $99 \%$ & $97 \%$ \\
\hline
\end{tabular}

Die Tabelle zeigt, dass nur wenige Haushalte glauben, dass ihre Kinder mit der Basisausbildung allein unabhängig werden können. Darüber hinaus glauben sie, dass auch für Arbeiten, die von unausgebildeten Personen ausgeführt werden könnten, ausgebildete Bewerber bevorzugt werden. Die meisten Eltern glauben also, dass der Arbeitsmarkt ihren gering ausgebildeten Kindern kaum Chancen bieten würde.

Die Basisausbildung stellt in Lima also aus individueller Sicht im Gesamtkalkül keinen privatwirtschaftlichen Wert dar. Vielmehr werden die Kosten der Ausbildung höher erwartet als ihre Nutzen. Dennoch bietet sich für die Familien keine Alternative zum Schulbesuch der Kinder, da befürchtet wird, dass diese sonst ihren Lebensunterhalt nicht aus dem Verkauf ihrer Arbeitskraft bestreiten können. Ein Ausweg aus diesem Dilemma scheint die höhere Ausbildung zu bieten, da erst dort deutlich höhere Einkommenssteigerungen erwartet werden. 


\subsubsection{Grenzbetrachtung der Rentabilitätserwartungen}

$\mathrm{Da}$ in der Bildungsökonomie die Investitionsentscheidung immer anhand von Grenzbetrachtungen vollzogen wird, soll auch im folgenden eine detaillierte Grenzbetrachtung durchgeführt werden, soweit es die Daten zulassen.

Wie schon erwähnt, wurden bisher nicht die jeweils zusätzlichen Bildungsstufen einzeln betrachtet, sondern der gesamte Bildungsweg einer Bewertung unterzogen. Während nach der Primaria mit hohen absoluten Verlusten gerechnet wird, werden diese durch den Abschluss der Sekundaria nur gering erhöht. Dies deutet darauf hin, dass die durch die Sekundaria zusätzlich erwarteten Kosten fast durch die erwarteten Einkommenssteigerungen ausgeglichen werden. Außerdem wurde ermittelt, dass sich die Gesamtrentabilität mit dem Abschluss der Sekundaria für alle Schichten erhöht. Demnach werden durchschnittlich größere Einkommens- als Kostenzuwächse durch die Sekundaria erwartet. Beide Beobachtungen weisen darauf hin, dass die Sekundaria in der Grenzbetrachtung deutlich besser abschneidet als die Primaria.

Analog zur Ertragsratenberechnung dürfen für eine Grenzbetrachtung der oben ermittelten Deckungsbeiträge nur die Differenzen der erwarteten Einkommen und der Rentabilitätseinkommen zwischen zwei Bildungsstufen verglichen werden. Für die Primaria reduziert sich der Deckungsbeitrag dadurch noch deutlich. Da auch ohne Bildung ein Einkommen erwartet wird, müssen hier zwar die gesamten Rentabilitätseinkommen, aber nur der Anstieg der erwarteten Einkommen betrachtet werden. Es ergeben sich Deckungsbeiträge um die 15\%.311

Für die Sekundaria müssen sowohl die erwarteten Einkommen als auch die Rentabilitätseinkommen auf den durch sie induzierten Teil reduziert werden. Es ergeben sich die folgenden Werte.

Tabelle 11.8: Einkommensänderungen durch den Besuch der Sekundaria

\begin{tabular}{l|rrrr}
\hline Änderungen durch den zusätzlichen Abschluss & \multicolumn{4}{c}{ Bevölkerungsschicht } \\
der Sekundaria & A & B & C & D \\
\hline Einkommensdifferenz & 356 & 337 & 262 & 131 \\
Differenz des Rentabilitätseinkommens & 388 & 350 & 289 & 180 \\
\hline „Deckungsbeitrag“ der Sekundaria & $92 \%$ & $96 \%$ & $91 \%$ & $73 \%$ \\
\hline
\end{tabular}

Wie die obigen Zahlen schon vermuten ließen, erwarten die Eltern in der Grenzbetrachtung für die Sekundaria deutlich niedrigere relative Verluste. Die Deckungsbeiträge liegen zwischen $73 \%$ und $96 \%$.

Sollen diese Werte als Ertragsraten ausgedrückt werden, müssen folgende Überlegungen angestellt werden: Wenn die Kinder genau die angegebenen Ren-

311 Da für die eigenen Kinder nicht nach Einkommen ohne Ausbildung gefragt wurde, wurden die allgemein am Markt erwarteten Einkommen herangezogen. Die Zahlen sind daher nur Näherungswerte. 
tabilitätseinkommen verdienen, ist der Kapitalwert der Bildungsinvestition bei gegebenem Zins gleich Null. Als Zins kann hier zum Beispiel der Marktzins angenommen werden. Verdienen die Kinder hingegen nur die von den Eltern erwarteten niedrigeren Einkommen, ist der Kapitalwert der Investition bei gleichem Zins negativ. Soll nun ein interner Zins ermittelt werden, der den Kapitalwert der Bildungsinvestition Null werden lässt, liegt dieser unter dem zum Vergleich herangezogenen Marktzins. Die Bildungsinvestition wird als nicht rentabel betrachtet. Die Ertragsrate der Primarbildung liegt weit unter dem Vergleichszins, während die Sekundarbildung relativ dicht darunter liegt. Das hier angewandte Vorgehen, aus spontanen Antworten der Eltern auf erwartete Ertragsraten zu schließen, kann natürlich wegen der ungenauen Angaben nicht zu exakten Kennzahlen führen. Dennoch konnte gezeigt werden, dass beide Investitionen aus wirtschaftlichen Gründen abzulehnen sind. Wenn die Investitionen am Kapitalmarkt finanziert werden müssten, wäre der dort zu zahlende Zins höher als die Ertragsrate der Ausbildung. Die Investition führt zu einem Verlust. Ebenso wäre eine Anlage des Betrages am Kapitalmarkt wirtschaftlicher.

Leider konnten keine Rentabilitätswerte für die höhere Bildung ermittelt werden, da hier keine Daten zu den Rentabilitätseinkommen vorliegen. Wird allerdings die erwartete Entwicklung der Einkommen und der als notwendig erachteten Rentabilitätseinkommen betracht, können Vermutungen dazu angestellt werden.

Wie schon erwartet, steigen die Rentabilitätseinkommen (die einen Hinweis auf die Kostenentwicklung geben) in allen Schichten geringer an als die erwarteten Einkommen.

Tabelle 11.9: Anstiegsraten mit dem Bildungsniveau

\begin{tabular}{l|l|cccc}
\hline \multicolumn{2}{l|}{ Steigerung der Rentabilitäseinkommen und } & \multicolumn{4}{|c}{ Bevölkerungsschicht } \\
Erwartungen durch den Besuch der Sekundarstufe & A & B & C & D \\
\hline Anstiegsraten & Erwartetes Einkommen & $81 \%$ & $90 \%$ & $83 \%$ & $40 \%$ \\
& Rentabilitätseinkommen & $37 \%$ & $43 \%$ & $44 \%$ & $33 \%$ \\
\hline
\end{tabular}

Während die als notwendig erachteten Rentabilitätseinkommen mit dem Abschluss der Sekundaria nur um 30-40\% wachsen, werden Einkommensanstiege bis $90 \%$ erwartet.

Wird nun auch für die höhere Bildung nur eine moderate Kostensteigerung angenommen, kann die große erwartete Einkommenssteigerung ${ }^{312}$ dazu führen, dass die Bildungsinvestition endlich rentabel wird. Die umfassende Nachfrage nach höherer Bildung lässt diesen Zusammenhang vermuten. Die Eltern schei-

312 Für die höhere Ausbildung wurden am Arbeitsmarkt durchschnittliche Einkommenszuwächse von bis zu 300\% erwartet. 
nen also auch bei der höheren Bildung zu erwarten, dass die Kostensteigerung deutlich hinter der Einkommenssteigerung zurück bleibt. Denn nur wenn durch die höhere Bildung so hohe Einkommens- und so niedrige Kostenzuwächse induziert werden, dass insgesamt auch die Verluste der Basisausbildung wieder ausgeglichen werden, kann aus ökonomischer Sicht der umfassende Wunsch nach weiterführender Bildung erklärt werden. Dazu müssten die Ertragsraten aber sehr hoch über dem Vergleichszins liegen.

\subsubsection{Zusammenfassung und Schlussfolgerungen}

Die Daten lassen interessante Schlüsse über das Bildungsnachfrageverhalten der Haushalte zu. Zunächst scheinen die Bildungsentscheidungen nicht anhand von marginalen Ertragsraten für die jeweils nächste Bildungsstufe getroffen zu werden. In diesem Fall müssten sich die meisten Eltern schon bei der Investitionsentscheidung für die Primaria gegen eine Einschulung entscheiden. Offensichtlich tun sie dies nicht. Da sich über $90 \%$ der Familien ein über die obligatorische Basisausbildung hinausgehendes Bildungsniveau für ihre Kinder wünschen, kann auch nicht argumentiert werden, dass die Bildungsbeteiligung allein durch die Schulpflicht bewirkt wird. Vielmehr scheint die Basisausbildung als notwendige Voraussetzung für die weitere Bildung betrachtet zu werden. Diese Erkenntnisse bestätigen also die empirischen Zusammenhänge aus einzelnen Studien, die im zweiten Teil 5 dargestellt wurden. ${ }^{313}$ Positive Erträge scheinen die Eltern erst bei Bildungsniveaus zu erwarten, die über die Basisausbildung hinausgehen. Nur so lassen sich die hohen Bildungsziele in der Mehrheit der Familien erklären. Die Basisausbildung allein führt nach Meinung fast aller Haushalte zu hohen absoluten Verlusten. Ein Kalkül scheint daher für die Gesamtausbildung aufgestellt zu werden, was in der bildungsökonomischen Literatur selten behandelt und als Durchschnittsrate bezeichnet wird. Mit der Durchschnittsrate wird das entsprechende Bildungsniveau mit der Alternative „keine Bildung“ verglichen. ${ }^{314}$ Ein solches Gesamtkalkül ist notwendig, um zu ermitteln, wann die Verluste der Basisausbildung durch weiterführende Schulbildung ausgeglichen sind.

Auch die bezüglich der Grenzertragsraten möglichen Aussagen zeigen, dass auch hier die in den 70er Jahren vor allem von PSACHAROPOULOS ermittelten Ertragsratenmuster ${ }^{315}$ in Frage gestellt werden müssen. Danach müsste in Entwicklungsländern die Primarbildung besonders hohe private Ertragsraten

\footnotetext{
313 Vgl z.B die Untersuchung von Lavy (1996), der zeigte, dass vor allem die Entfernung zu Post-Primary Schulen eine große Rolle bei der Einschulungsentscheidung und bei den Erfolgen der Primaria spielt.

314 Vgl. dazu die Unterscheidung der beiden Ertragsratenarten in Abschnitt 2.1.4.

315 Vgl. Abschnitt 2.3.
} 
aufweisen. Wie oben gezeigt wurde, wird diese Relation auch für Entwicklungsländer mittlerweile bezweifelt. Die Eltern scheinen die sinkende Relevanz der Basisausbildung für den Erfolg am Arbeitsmarkt erkannt zu haben. In ihrem privaten Kalkül hat diese Ausbildungsstufe jedenfalls keinen ökonomischen Wert. Auch direkt nach den Chancen am Arbeitsmarkt gefragt, bezweifeln sie den Wert der Basisausbildung.

Hier sei nochmals darauf hingewiesen, dass die in der Theorie benutzten Raten aus der Sicht von Ökonomen unter Berücksichtigung umfangreicherer Informationen aufwendig berechnet werden. Die hier untersuchten individuellen Kalküle können aufgrund von Informationsdefiziten oder Fehleinschätzungen der Arbeitsmarktsituation zu abweichenden Ergebnissen führen.

Diese individuellen Erwartungen (ob sie realistisch sind oder nicht) sind es aber, die die private Bildungsnachfrage beeinflussen. Daher sollte den obigen Erkenntnissen in der Bildungspolitik unbedingt Bedeutung beigemessen werden. Nur wenn diese Mechanismen bekannt sind, können die richtigen Instrumente identifiziert werden, um die festgesteckten Ziele der Bildungspolitik zu erreichen.

Eine Ausweitung des Bildungsangebots wird die Bildungsbeteiligung und die Leistungen in der Basisausbildung nicht erhöhen, wenn nicht gleichzeitig der Zugang zu höheren Bildungseinrichtungen ermöglicht und kommuniziert wird. Ebenso wäre es aus bildungspolitischen Gründen wichtig zu wissen, ob die Erwartungen der Individuen realistisch sind oder nicht. ${ }^{316}$ Ist die schlechte Bewertung der Basisausbildung tatsächlich das Resultat von Informationsdefiziten, kann die Bildungspolitik u. U. allein mit Informationsverbesserung das Nachfrageverhalten der Individuen korrigieren. Basieren die Erwartungen dagegen auf realistischen Werten, ist zu hinterfragen, ob eine umfassende Basisausbildung überhaupt erstrebenswert ist. Für Kinder, die ihre Ausbildung nicht an einer höheren Bildungseinrichtung fortführen können, kann die Verpflichtung zum Abschluss der Basisausbildung zu hohen privaten Verlusten führen. In dem Fall, in dem die externen Nutzen der Basisausbildung eine Schulpflicht rechtfertigen, sollte die Bildungsteilnahme dieser Kinder subventioniert werden.

Für die Bildungspolitik ist die Kenntnis der hier behandelten Ertragserwartung von immens großer Bedeutung. Zum einen haben sie direkten Einfluss auf die Wirkung von bestehenden bildungspolitischen Maßnahmen, zum anderen sollten sie Einfluss auf die Auswahl dieser Maßnahmen haben. Dabei ist die

316 Vgl. zu der Erwartungsbildung die Ausführungen in Abschnitt 4.1 und zu den Schätzungen der zukünftigen Einkommensentwicklung die Ausführungen in Abschnitt 6.2. Die Untersuchungen lassen darauf schließen, dass die Einkommenserwartungen zumindest von US-amerikanischen Schülern der höheren Bildungsstufen sehr realistisch ausfallen. 
Kenntnis der Realitätsnähe der Erwartungen unabdingbar. Wie die Ausführungen in Teil II gezeigt haben, besteht aber gerade hier - und besonders für Entwicklungsländer - enormer Forschungsbedarf.

\subsubsection{Unterschiede in den Ertragserwartungen nach Schichten}

Wie die obigen Tabellen zu den Ertragserwartungen zeigten, fallen die Erwartungen in den oberen Schichten im Durchschnitt deutlich optimistischer aus als in den unteren Schichten. Unterschiede zeigen sich sowohl in der Schätzung für den Arbeitsmarkt als auch für die eigenen Kinder. Die unterschiedlichen Einkommenserwartungen wirken sich natürlich auf die Rentabilität der Ausbildung aus. Deshalb werden hier zunächst die Unterschiede in den Einkommenserwartungen genauer untersucht, bevor in einem nächsten Schritt die Angaben über das Rentabilitätseinkommen ausgewertet werden. Die sich daraus ergebenden Unterschiede in der erwarteten Rentabilität der Basisausbildung werden wie oben in der Durchschnitts- und der Grenzbetrachtung aufgezeigt.

\subsubsection{Einkommenserwartungen am Arbeitsmarkt}

Um die Unterschiede in den Einkommenserwartungen zwischen den Schichten aufzeigen zu können, wird die Tabelle 11.2 aus Abschnitt 11.2 noch einmal herangezogen.

Tabelle 11.2: Erwartete Einkommensdifferenzen nach Bildungsniveaus

\begin{tabular}{l|rrrr}
\hline Monatl. Einkommen einer Person & \multicolumn{4}{|c}{ Bevölkerungsschicht } \\
(Durchschnittswerte in Soles) & A & B & C & D \\
\hline ohne Ausbildung & 301 & 317 & 284 & 268 \\
mit Primaria* $^{*}$ & 421 & 415 & 395 & 345 \\
mit Secundaria* $_{\text {mit höherer Bildung* }}$ & 689 & 656 & 575 & 464 \\
\hline
\end{tabular}

*Varianzanalyse: Unterschiede sind signifikant mit einer Irrtumswahrscheinlichkeit gegen Null.

Wie die Daten zeigen, wird das mögliche Einkommen am Arbeitsmarkt für eine Person ohne eine abgeschlossene Ausbildung von den Familien der verschiedenen Schichten im Durchschnitt noch relativ ähnlich eingeschätzt. Das geschätzte Einkommen liegt in allen Schichten bei ca. 300 Soles im Monat. Allerdings klaffen die Schätzungen zwischen den Schichten mit steigenden Bildungsabschlüssen immer weiter auseinander. Während Haushalte der obersten Schicht im Schnitt ca. 3000 Soles für eine Person mit höherer Bildung erwarten, glaubt der durchschnittliche Haushalt der untersten Schicht nur noch an einen Monatsverdienst von knapp 1000 Soles.

Die Erwartungsunterschiede zwischen den Schichten sind signifikant schichtspezifisch. Diese Ergebnisse lassen sich anhand der Varianzanalyse bestätigen. Der Mittelwertvergleich zeigt, dass die Einkommenserwartungen in der 
Gruppenzugehörigkeit begründet liegen. Die Ergebnisse sind ab der Primaria signifikant (bei einem Niveau von 99\%). Die Hypothese, dass es sich um zufällige Diskrepanzen handelt kann daher abgelehnt werden. Die Irrtumswahrscheinlichkeit sinkt sogar noch mit steigendem Bildungsniveau, während das Bestimmtheitsmaß steigt. ${ }^{317}$ Die großen Erwartungsunterschiede können als in der Schichtzugehörigkeit begründet angenommen werden.

Die Haushalte der obersten Schichten erwarten auf jeder Bildungsstufe deutlich höhere Einkommen als die Haushalte der unteren Schichten. Sie trauen der Ausbildung außerdem wesentlich höhere Einkommenssteigerungen zu. Während die Haushalte der obersten Schicht von der höheren Bildung einen Anstieg der monatlichen Einkommen um 324\% erwarten, sind dies bei den Haushalten der untersten Schicht nur 107\%.318

Welche relativen geschätzten Einkommensunterschiede sich daraus ergeben, wird in der folgenden Tabelle zusammengefasst. Dazu wurden die Einkommenserwartungen der drei unteren Schichten als Prozentsatz der Erwartungen der obersten Schicht ausgedrückt.

Tabelle 11.10: Erwartete Einkommen nach Bildungsniveaus

\begin{tabular}{l|rrrc}
\hline Einkommenserwartungen als \% der & \multicolumn{4}{|c}{ Bevölkerungsschicht } \\
Erwartungen der obersten Schicht & A & B & C & D \\
\hline ohne Ausbildung & $100 \%$ & $105 \%$ & $94 \%$ & $89 \%$ \\
mit Primaria & $100 \%$ & $99 \%$ & $94 \%$ & $82 \%$ \\
mit Secundaria & $100 \%$ & $95 \%$ & $83 \%$ & $67 \%$ \\
mit höherer Bildung & $100 \%$ & $65 \%$ & $48 \%$ & $33 \%$ \\
\hline
\end{tabular}

Obwohl bei jeder Bildungsstufe die geschätzten Einkommen mit dem sozioökonomischen Niveau der Haushalte sinken, ist diese Diskrepanz bei der höheren Bildung am deutlichsten ausgeprägt. Die Haushalte der untersten Schicht erwarten hier nur noch ein Drittel der Einkommen, die von der obersten Schicht erwartet werden.

In diesem ersten Schritt wurden die Haushalte gefragt, wie sie die Verdienste für Personen mit unterschiedlichen Einkommen am Arbeitsmarkt allgemein einschätzen. Die Schätzungen müssten sich also auf immer dieselbe Durchschnittsperson beziehen. Die großen Unterschiede in den Erwartungen zeigen, dass die Einschätzungen nicht auf rationalen Erwartungen beruhen können. Wären die Erwartungen rational, müssten sie dicht an den tatsächlichen Einkommen liegen und damit gleichmäßiger ausfallen. Wie die Ausführungen zur Erwartungsbildung in Kapitel 4 zeigten, ist anzunehmen, dass Erwartungen zum

317 Die genauen Daten sind dem Anhang, Tabellen A9 und A10, zu entnehmen. Vgl. zur Varianzanalyse auch Zucchini/Sattler (2000) und Backhaus/Erichson/Plinke/Schuchard-Ficher/Weiber (1987), S.4366.

318 Vgl. die Tabelle 11.3 in Abschnitt 11.2. 
Teil auch immer aufgrund von eigenen Erfahrungen gebildet werden. Wie die Auswertungen der Einkommensverhältnisse in den verschiedenen Schichten zeigte, machen Familien in verschiedenen Schichten auch verschieden hohe Einkommenserfahrungen. Die Diskrepanz in den Erwartungen ist damit also z. T. erklärbar.

Die These der z. T. adaptiven Erwartungsbildung wird auch dadurch unterstützt, dass die Diskrepanzen bei den Einkommenserwartungen für die höhere Bildung am größten sind. Dies war zu erwarten, da den Haushalten der unteren Schichten in diesem Bereich die Erfahrungen fehlen und sie deshalb die Einkommen unterschätzen.

Bei diesen allgemein am Arbeitsmarkt für realistisch betrachteten Einkommen, sollten auch Unterschiede in der Art der absolvierten Bildungsabschlüsse keine große Rolle spielen. Allerdings zeigte die obige Untersuchung, dass fast alle Familien der obersten Schicht private Schulen für ihre Kinder aussuchten, während die Kinder der untersten Schicht zum größten Teil öffentliche Schulen besuchten. Der Einfluss auch dieser Erfahrung auf die Schätzung der allgemeinen Einkommen kann nicht ganz ausgeschlossen werden.

Die unterschiedlichen Chancen der eigenen Kinder am Arbeitsmarkt sollten erst durch die folgende Fragestellung aufgedeckt werden.

\subsubsection{Einkommenserwartungen für die eigenen Kinder}

Um die Einkommenserwartungen zwischen den Schichten zu vergleichen, wird noch einmal ein Teil der schon aus Abschnitt 11.4.1.1 bekannten Tabelle 11.4 herangezogen.

\section{Auszug aus Tabelle 11.4: Einkommenserwartungen für die eigenen Kinder}

\begin{tabular}{|c|c|c|c|c|c|}
\hline \multirow{2}{*}{\multicolumn{2}{|c|}{$\begin{array}{l}\text { Monatl. Einkommen einer Person ... } \\
\text { (Durchschnittswerte in Soles) }\end{array}$}} & \multicolumn{4}{|c|}{ Bevölkerungsschicht } \\
\hline & & A & B & C & D \\
\hline \multirow[t]{2}{*}{ erwartete Einkommen } & Primaria* & 442 & 376 & 317 & 327 \\
\hline & Sekundaria* & 798 & 713 & 579 & 458 \\
\hline
\end{tabular}

Sie gibt die monatlichen Einkommen an, die für die eigenen Kinder mit alternativen Bildungsniveaus erwartet würden, wenn diese die Schule nach dem entsprechenden Abschluss verlassen würden. Die Erwartungen unterscheiden sich deutlich. Die Unterschiede zwischen den Schichten sind signifikant mit einer Irrtumswahrscheinlichkeit gegen Null. ${ }^{319}$ Auch hier kann also die Hypothese, dass es sich um zufällige Diskrepanzen handelt, abgelehnt werden.

319 Die genauen Daten sind dem Anhang, Tabellen A9 und A10, zu entnehmen. Vgl. zur Varianzanalyse auch Zucchini/Sattler (2000) und Backhaus/Erichson/Plinke/Schuchard-Ficher/Weiber (1987), S.4366. 
Werden die gleichen Relationen wie im vorangehenden Abschnitt für die Einkommenserwartungen für die eigenen Kinder berechnet, zeigt sich, dass die Unterschiede hier tatsächlich noch deutlicher ausfallen als am Arbeitsmarkt allgemein. ${ }^{320}$

Tabelle 11.11: Erwartete Einkommen nach Schichten

\begin{tabular}{l|cccc}
\hline Einkommenserwartungen als \% der & \multicolumn{4}{|c}{ Bevölkerungsschicht } \\
Erwartungen der obersten Schicht & A & B & C & D \\
\hline mit Primaria & $100 \%$ & $85 \%$ & $72 \%$ & $74 \%$ \\
mit Secundaria & $100 \%$ & $89 \%$ & $73 \%$ & $57 \%$ \\
\hline
\end{tabular}

Diese Ergebnisse kommen dadurch zustande, dass die Haushalte der oberen Schichten glauben, dass die Einkommen ihrer eigenen Kinder über denen liegen, die allgemein am Arbeitsmarkt erwartet werden. Die Familien der untersten Schicht hingegen glauben, dass ihre Kinder im Schnitt weniger verdienen als allgemein am Arbeitsmarkt üblich. Diese Diskriminierungserwartungen sind bei der Sekundaria noch deutlicher ausgeprägt als bei der Primaria. Die Tabelle zeigt die durchschnittlichen Unterschiede in den einzelnen Schichten.

Tabelle 11.12: Diskriminierung der eigenen Kinder am Arbeitsmarkt

\begin{tabular}{l|rrrr}
\hline erwartetes Einkommen für die eigenen Kinder & \multicolumn{4}{|c}{ Bevölkerungsschicht } \\
\cline { 2 - 5 } abzüglich des erwarteten Einkommens am Markt & A & B & C & D \\
\hline Primaria & 20,43 & 39,41 & $-77,92$ & -18 \\
\hline Secundaria & 109,4 & 56,55 & 3,44 & $-6,38$ \\
\hline
\end{tabular}

Für ihre Kinder mit Sekundarschulabschluss haben die Eltern der obersten Schicht im Durchschnitt 109 Soles mehr angegeben als sie üblicherweise am Arbeitsmarkt erwarten. Die Eltern der untersten Schicht gaben im Gegensatz dazu für ihre eigenen Kinder 6 Soles weniger an.

Neben den o. g. Unterschieden in der Erwartungsbildung aus Erfahrungswerten können für die eigenen Kinder noch weitere Faktoren die Unterschiede erklären. Die höheren Erwartungen der Haushalte der oberen Schichten könnten zum Beispiel daher rühren, dass sie annehmen, ihren Kindern eine qualitativ höherwertigere Ausbildung als der Durchschnitt geboten zu haben. Hier sollte sich jetzt das Vertrauen der Eltern in die Qualität der gewählten privaten Bildungseinrichtung zeigen. Außerdem könnten sie ihren Kindern bei der Jobsuche behilflich sein, indem sie zum Beispiel aus den eigenen Positionen heraus Beziehungen spielen lassen oder Erfahrungen für Bewerbungsverfahren aufweisen können. Die bessere ökonomische Situation der Haushalte könnte den Kindern längere Suchprozesse ermöglichen. Aber auch die, in der eigenen Familie erlangte, Selbstsicherheit und Vertrautheit mit dem Habitus der potentiel-

320 Vgl. dazu die Tabelle 11.2 im vorangegangenen Abschnitt 11.2. 
len Arbeitgeber kann entscheidenden Einfluss auf die Bewerbungsgespräche und Lohnverhandlungen haben.

Die Unterschätzung der Einkommenschancen der eigenen Kinder in den unteren Schichten könnten darin begründet liegen, dass sich die Eltern der Vorteile der oberen Schichten bewusst sind. Andererseits könnten sie auch einfach eine Diskriminierung der eigenen Kinder am Arbeitsmarkt annehmen.

Inwiefern die unterschiedlichen Erwartungen den Bedingungen am Arbeitsmarkt entsprechen, kann hier nicht beantwortet werden. Auf jeden Fall werden die unterschiedlichen Erwartungen über die Einkommen am Arbeitsmarkt noch durch die Erwartungen für die eigenen Kinder verstärkt. Für die Nachfrage nach Ausbildung ist allerdings die Rentabilität der Ausbildung von Relevanz. Deshalb wird im nächsten Abschnitt untersucht, inwieweit sich die Ansprüche an das Rentabilitätseinkommen unterscheiden.

\subsubsection{Ansprüche an die Rentabilitätseinkommen}

Wie schon die Tabelle 11.4 in Abschnitt 11.4.1.1 gezeigt hat, unterscheiden sich neben den Einkommenserwartungen auch die genannten Rentabilitätseinkommen zwischen den Schichten.

\section{Auszug aus Tabelle 11.4: Rentabilitätserwartungen für die eigenen Kinder}

\begin{tabular}{l|l|rrrr}
\hline \multicolumn{2}{l|}{$\begin{array}{l}\text { Monatl. Einkommen einer Person ... } \\
\text { (Durchschnittswerte in Soles) }\end{array}$} & \multicolumn{4}{|c}{ Bevölkerungsschicht } \\
\hline Rentabilitätseinkommen & Primaria* $^{*}$ & 1.059 & 810 & 659 & 548 \\
& Sekundaria* $^{*}$ & 1.447 & 1.160 & 948 & 728 \\
\hline
\end{tabular}

*Varianzanalyse: Unterschiede sind signifikant mit einer Irrtumswahrscheinlichkeit gegen Null.

Auch hier sind die Unterschiede zwischen den Schichten hochsignifikant. ${ }^{321}$

321 Die genauen Daten sind dem Anhang, Tabellen A9 und A10, zu entnehmen. Vgl. zur Varianzanalyse auch Zucchini/Sattler (2000) und Backhaus/Erichson/Plinke/Schuchard-Ficher/Weiber (1987), S.4366. 
Werden die Angaben wieder in Relation zu den Beträgen der obersten Schicht ausgedrückt, ergibt sich das folgende Bild.

Tabelle 11.13: Erwartete Rentabilitätseinkommen nach Schichten

\begin{tabular}{l|cccc}
\hline Rentabilitätseinkommen als \% der & \multicolumn{4}{|c}{ Bevölkerungsschicht } \\
Erwartungen der obersten Schicht & A & B & C & D \\
\hline mit Primaria & $100 \%$ & $76 \%$ & $62 \%$ & $52 \%$ \\
mit Secundaria & $100 \%$ & $80 \%$ & $66 \%$ & $50 \%$ \\
\hline
\end{tabular}

Mit sinkendem sozioökonomischen Niveau wurden auch niedrigere Rentabilitätseinkommen angegeben. In der untersten Schicht werden die Bildungsinvestitionen schon ausgeglichen, wenn die Kinder nur die Hälfte von dem verdienen, was in der obersten Schicht als notwendig erachtet wurde.

Wird auch hier wieder davon ausgegangen, dass das Rentabilitätseinkommen ohne die Berücksichtigung von Zinsen ${ }^{322}$ ermittelt wurde, kann aus diesen Angaben geschlossen werden, dass die Haushalte der oberen Schichten auch höhere Kosten der Ausbildung aufweisen. Bei gleichem Bildungsniveau investiert die Durchschnittsfamilie der obersten Schicht also deutlich mehr als die Durchschnittsfamilie der untersten Schicht. Auch hier muss wieder berücksichtigt werden, dass die Rentabilitätseinkommen nicht anhand einer Barwertermittlung berechnet wurden. Es handelt sich bei den Angaben um spontane Ideen der Eltern. Schlüsse auf die tatsächlichen Investitionshöhen lassen sich damit nicht ziehen. Einen Vergleich zwischen den Schichten lassen die Daten aber zu, da sich alle Haushalte spontan zu den Fragen äußern mussten.

Die angegebenen Kostenunterschiede sind durchaus als realistisch zu betrachten, da fast alle Kinder der obersten Schicht private Schulen und fast alle Kinder der untersten Schicht öffentliche Schulen besuchen. ${ }^{323}$

Wird nun die Nicht-Berücksichtigung der Zinsen aufgegeben, zeigt sich, dass die obigen Erkenntnisse sogar noch verstärkt werden. Da davon ausgegangen werden muss, dass der Kapitalmarkt für Bildungsinvestitionen beschränkt ist und reichere Haushalte besseren Finanzierungsbedingungen ausgesetzt sind als arme Haushalte, müssten die reicheren Familien auch niedrige Zinsen für die Berechnung von Rentabilitätseinkommen ansetzen. Dann müssten die Haushalte der obersten Schicht c. p. auch niedrigere Rentabilitätseinkommen berechnen, da sie ihre Kosten nicht so stark auf- und die Nutzen nicht so stark abzinsen müssen. Die Daten zeigen aber, dass sie höhere Angaben gemacht haben als die Haushalte der unteren Schichten. Das heißt, trotz des gegenläufigen Effektes der Zinsen überwiegt der Kosteneffekt. Das wiederum bedeutet,

322 Oder bei Annahme nur eines gültigen Zinsniveaus für alle Haushalte.

323 Vgl. dazu die Ausführungen über die Unterschiede in den Bildungsgewohnheiten in Abschnitt 10.2. 
dass die Unterschiede in den Investitionshöhen noch deutlicher ausfallen als der Vergleich der Rentabilitätseinkommen vermuten lässt. Deshalb können die Unterschiede in den Rentabilitätseinkommen im folgenden durchaus als Indikator für Unterschiede in den Investitionshöhen betrachtet werden.

Vergleicht man die Raten der Einkommenserwartungen mit denen der Rentabilitätseinkommen, wird festgestellt, dass die Rentabilitätseinkommen mit dem sozioökonomischen Niveau der Haushalte stärker sinken als die Einkommenserwartungen. Die Erwartungen über die Rentabilität der Bildungsstufen müssten in den unteren Schichten also optimistischer ausfallen als in den oberen Schichten. Die Erwartungen über die Rentabilität der Ausbildung wird im folgenden Abschnitt untersucht.

\subsubsection{Gesamtrentabilität der Bildungsstufen}

Wie die beiden vorangehenden Abschnitte gezeigt haben, erwarten die Eltern der oberen Schichten nicht nur höhere Einkommen, sie gaben auch jeweils höhere Rentabilitätseinkommen an. Das heißt, sie erwarten auch höhere Investitionskosten bei jedem Bildungsniveau. Um vergleichende Aussagen zu den Rentabilitätserwartungen machen zu können, müssen daher diese beiden Größen verglichen werden. Auch dazu werden wieder einige Daten aus bereits dargestellten Tabellen herangezogen. Zunächst wird die Höhe der absoluten Verluste zwischen den Haushalten der verschiedenen Schichten verglichen. Die Haushalte der oberen Schichten erwarten deutlich höhere absolute Verluste als die der unteren Schichten, wenn ihre Kinder nach dem Abschluss der Basisausbildung (also nach der Sekundaria) die Schule verließen. Während die Haushalte der obersten Schicht einen durchschnittlichen Verlust von ca. 650 Soles erwarten, sind dies in der untersten Schicht nur 270 Soles. ${ }^{324}$

Wird das erwartete Einkommen in Relation zum Rentabilitätseinkommen betrachtet, ergeben sich die folgenden Werte, die bereits in Abschnitt 11.4.1.1 dargestellt wurden.

Tab. 11.5: Erwartete Einkommen als \% der Rentabilitätseinkommen

\begin{tabular}{l|c|cccc}
\hline Erwartete Einkommen als \% der & Stichpr. & \multicolumn{4}{|c}{ Bevölkerungsschicht } \\
Rentabilitätseinkommen & gesamt & A & B & C & D \\
\hline Eink. Prim./Rentabilitätseinkommen & $50 \%$ & $42 \%$ & $46 \%$ & $48 \%$ & $60 \%$ \\
Eink. Sec./Rentabilitätseinkommen & $61 \%$ & $55 \%$ & $61 \%$ & $61 \%$ & $63 \%$ \\
\hline
\end{tabular}

Die Zahlen zeigen, dass sowohl die Primaria als auch die Sekundaria von den Familien der untersten Schicht weniger verlustreich eingeschätzt wurde als von den Familien der oberen Schichten. Sie erwarten immerhin den Rückfluss von 
$60 \%$ bzw. 63\% ihrer Investitionen bei beiden Bildungsstufen. Die Familien der obersten Schicht trauen der Primaria nur einen Rückfluss von gut $40 \%$ zu; von der Sekundaria erwarten sie immerhin einen Rückfluss von 55\%.

Die Gesamtrentabilität der Basisausbildung wird von den Haushalten der unteren Schichten also optimistischer eingeschätzt. Sowohl die absoluten Verluste als auch die Verluste in Relation zur eingesetzten Investitionssumme wurden von den Haushalten der oberen Schichten höher eingeschätzt. Hier ist nochmals zu betonen, dass von den Rentabilitätseinkommen nicht auf die absoluten Investitionsmengen geschlossen werden kann. Ein Vergleich der beiden Größen ist aber möglich. 325

Die Tabelle zeigt aber auch, dass die Zuversicht mit dem Bildungsniveau in den oberen Schichten stärker ansteigt. Nach Abschluss der Sekundaria werden nach Ansicht der obersten Schicht schon 55\% der gesamten Bildungsinvestition abgedeckt. Das ist eine Steigerung von 13 Prozentpunkten. Die Deckungsrate steigt bei der untersten Schicht hingegen nur um 3 Prozentpunkte auf 63\%. Offensichtlich wird die Sekundaria in der Grenzbetrachtung von den Haushalten der oberen Schichten als rentabler betrachtet als von den Haushalten der untersten Schicht. Diese Vermutung wird im folgenden genauer untersucht.

\subsubsection{Grenzbetrachtung der Rentabilitätserwartungen}

Für eine Grenzbetrachtung der Sekundarausbildung wird die ebenfalls schon aus Abschnitt 11.4.1.2 bekannte Tab. 11.9 herangezogen. Sie wird im folgenden nochmals dargestellt und zeigt den durch die Sekundaria erwarteten Einkommensanstieg und den dazugehörigen Anstieg der Rentabilitätseinkommen.

Tab. 11.9: Anstiegsraten mit dem Bildungsniveau, Sekundaria

\begin{tabular}{|c|c|c|c|c|c|}
\hline \multirow{2}{*}{\multicolumn{2}{|c|}{$\begin{array}{l}\text { Steigerung der Einkommen und Erwartungen } \\
\text { durch den Besuch der Sekundarstufe }\end{array}$}} & \multicolumn{4}{|c|}{ Bevölkerungsschicht } \\
\hline & & A & B & C & D \\
\hline \multirow[t]{2}{*}{ Anstiegsraten } & Erwartetes Einkommen & $81 \%$ & $90 \%$ & $83 \%$ & $40 \%$ \\
\hline & Rentabilitätseinkommen & $37 \%$ & $43 \%$ & $44 \%$ & $33 \%$ \\
\hline
\end{tabular}

Während die erwarteten Einkommenssteigerungen durch den Abschluss der Sekundaria zwischen den Schichten sehr ungleichmäßig ausfallen, wurde der Anstieg der als notwendig erachteten Rentabilitätseinkommen wesentlich gleichmäßiger eingeschätzt. Der notwendige monatliche Einkommensanstieg, um die Investition auszugleichen, liegt zwischen 33\% und 44\% und zeigt kein deutliches von der Schicht abhängendes Muster.

325 Vgl. die Definition der Rentabilitätseinkommen in 11.3 und die obigen Ausführungen. 
Dem in allen Schichten etwa gleich großen Kostenanstieg durch den Besuch der Sekundaria steht bei der obersten Schicht ein erwarteter Einkommensanstieg von $80 \%$ gegenüber. Die Haushalte der untersten Schicht trauen der Sekundaria dagegen nur eine Einkommenssteigerung um 40\% zu. Diese Entwicklung gibt eine Hinweis darauf, dass die Sekundaria in der Grenzbetrachtung für die Haushalte der oberen Schicht besser abschneidet.

Diese Vermutung wird bestätigt, wenn man sich die erwarteten Deckungsbeiträge der Sekundaria in der Grenzbetrachtung anschaut. ${ }^{326}$ Hier zeigt sich, dass der ökonomische Wert der Sekundaria für sich betrachtet in den oberen Schichten deutlich höher angenommen wird als in der untersten Schicht. Die Haushalte der obersten Schicht erwarten, dass die Sekundaria zu über 90\% der notwendigen Einkommenssteigerungen führt, die für den Ausgleich der Investition notwendig wären. In der untersten Schicht werden nur knapp 75\% der dazu notwendigen Einkommen für realistisch gehalten.

Übersetzt in Ertragsraten heißt dies, dass zwar die Ertragsrate für die Sekundaria in allen Schichten unter dem jeweils angenommenen Vergleichszins liegt, die Ertragsrate der obersten Schicht aber dichter an ihrem Zins liegt als die der untersten Schicht an ihrem.

Wird für alle Schichten derselbe Zins angenommen, liegen die Ertragsraten der obersten Schicht über denen der unteren. Werden allerdings unterschiedliche Zinsen angenommen, kann eine eindeutige Aussage über die Lage der Ertragsraten zueinander nicht mehr getroffen werden.

\subsubsection{Zusammenfassung und Schlussfolgerungen}

Obwohl die Haushalte der oberen Schichten die Basisausbildung insgesamt verlustreicher einschätzen als die Haushalte der unteren Schichten, erwarten sie höhere Rentabilitätssteigerungen mit steigendem Bildungsniveau. Der Grund dafür ist, dass alle Haushalte eine ähnliche Kostenentwicklung erwarten, aber die Haushalte der untersten Schicht Einkommenssteigerungen erwarten, die weit hinter den Erwartungen der Haushalte der oberen Schichten zurückbleiben. Ursache für die Unterschiede in der erwarteten Rentabilitätsentwicklung sind demnach nicht die Erwartungen über die Kostenentwicklung, sondern vor allem die unterschiedlich eingeschätzten Auswirkungen der Bildung auf die Einkommen.

Der Unterschied in der einkommensteigernden Wirkung der Ausbildung ist allerdings erst bei der höheren Bildung am größten. Setzt sich die relativ einheitlich Entwicklung der Kosten für die höhere Bildung fort, ist zu erwarten, dass die 
Ertragsraten der Familien der oberen Schichten bei der höheren Bildung dann über den der Familien der unteren Schichten liegen. Wie bereits erwähnt, muss angenommen werden, dass die höhere Bildung die gesamte Bildungsinvestition rentabel werden lässt. Das heißt, dass in der Grenzbetrachtung sehr hohe Ertragsraten erwartet werden müssen.

Was diese Erkenntnisse für die Bildungsnachfrage der unterschiedlichen Bevölkerungsschichten bedeuten können, wird im vierten Teil anhand der grafischen Analyse behandelt. 


\subsection{Zusammenfassung der Wünsche und Einkommenserwar- tungen}

Zusammenfassend lässt sich zunächst feststellen, dass fast alle Eltern sich ein höheres, über die obligatorische Basisausbildung hinausgehendes, Bildungsniveau für ihre Kinder wünschen. Weiterhin halten fast alle Eltern die Basisausbildung für nicht rentabel. Für diese Ausbildung allein erwarten die Eltern sogar hohe Verluste.

Was der vornehmliche Grund für die breite Partizipation an der Basisausbildung ist, kann nicht eindeutig beantwortet werden. Einerseits ist es möglich, dass die Eltern ein Investitionskalkül für die höhere Bildung aufstellen und die Bildung dann insgesamt als rentabel betrachten. Der Wert der Basisausbildung liegt dann nicht im dort angeeigneten Humankapital und dessen Umsetzung in höhere Einkommen, sondern in der Zugangsvoraussetzung für höhere Bildung. Andererseits ist es ebenso möglich, dass sie zunächst kein Investitionskalkül aufstellen, sondern ihre Kinder wegen der Schulpflicht in die Basisausbildung schicken. Die Entscheidung allein für die Basisausbildung basiert in beiden Fällen nicht auf einem ökonomischen Kalkül. Im ersten Fall wird sie als notwendige Voraussetzung für jede weitere Bildung und im zweiten Fall als Pflichtveranstaltung betrachtet. In beiden Fällen muss der höheren Ausbildung eine so große Rentabilität zugetraut werden, dass die Verluste durch die Basisausbildung ausgeglichen werden. Die Basisausbildung für sich genommen hat aus Sicht der Eltern keinen ökonomischen Wert. Erst mit der Option, anschließend Zugang zu einer höheren Bildungseinrichtung zu haben, wird der Basisausbildung überhaupt ein Wert zuerkannt.

Diese Erkenntnis ist für die Erklärung der Bildungsnachfrage von großer Bedeutung. Damit wird nämlich der rentable Zugang zu weiterführenden Bildungseinrichtungen zu einer wichtigen Determinante für die Bildungsnachfrage. Dieser Zusammenhang wird auch durch einige empirische Studien belegt. ${ }^{327}$ Ist die Aussicht auf eine Fortführung des Bildungsweges von Anfang an schlecht, wird die Basisausbildung als notwendiges Übel betrachtet. Auch wenn die Kinder dennoch aus Pflichtbewusstsein in die Schule geschickt werden, wird sich die niedrige Wertschätzung der Eltern auf die Wahrnehmung der Kinder und ihre Unterstützung und damit auf deren Leistungen auswirken. Die Annahme, dass die Basisausbildung unrentabel ist, kann demnach nicht nur zu Nicht-Teilnahme

327 Vgl. dazu die Auswertung der empirischen Studien zu den Bildungsdeterminanten. Lavy hat ermittelt, dass die Entfernung zu weiterführenden Schulen durchaus einen Einfluss auf die Einschulungsentscheidung in Ghana hat. 
oder Schulabbruch führen, sondern auch die Leistungen der eingeschulten Kinder erheblich beeinflussen.

Wirklich hohe Einkommenssteigerungen erwarten die Eltern aller sozioökonomischen Schichten erst bei Abschluss einer höheren Bildungsstufe. Die höhere Bildung muss nicht nur in der Grenzbetrachtung rentabel sein, sie muss auch die Verluste der notwendigen Basisausbildung ausgleichen. Ein Investitionskalkül beginnt daher erst bei der höheren Bildung, um das Bildungsausmaß zu identifizieren, das den Nutzen aus der Gesamtausbildung maximiert.

Auffällig ist außerdem die Erkenntnis, dass Familien aus den oberen sozioökonomischen Schichten die Möglichkeit der Einkommenssteigerung - vor allem bei der höheren Bildung - deutlich optimistischer einschätzen als die Familien aus unteren Schichten. Bei ähnlich angenommenen Kostenverläufen folgt daraus, dass die erwarteten Ertragsraten der Haushalte der oberen Schichten deutlich über denen der Haushalte der unteren Schichten liegen. Allerdings zeigen die Daten auch, dass die Familien unterschiedlich hohe Investitionsbeträge in die Ausbildung ihrer Kinder einsetzen. Die höheren Ausgaben der Familien der oberen Schichten führen dazu, dass sie die Basisausbildung in der Durchschnittsbetrachtung als verlustreicher einschätzen als die Haushalte der unteren Schichten. Allerdings zeigt sich auch, dass die Haushalte der oberen Schichten deutlich optimistischer sind, was die Rentabilitätsentwicklung mit steigendem Bildungsniveau betrifft.

Inwieweit diese Erwartungen realistisch sind oder nicht spielt für die Untersuchung der Nachfragedeterminanten keine Rolle. Sollen allerdings Schlüsse für die Bildungspolitik gezogen werden, kann diese Information von großer Bedeutung sein.

Wie diese Erkenntnisse in die grafische Darstellung integriert werden können, und was sie für die optimalen Bildungsniveaus der Familien der verschieden sozioökonomischen Schichten bedeuten, wird im vierten Teil dargestellt. Zunächst folgen noch einige Erwartungen und Einstellungen der Eltern gegenüber der Schule, die über die reinen Rentabilitätserwartungen hinaus gehen. 


\section{Sonstige Einstellungen gegenüber der Ausbildung}

Nachdem die Ertragserwartungen der Basisausbildung ausführlich dargestellt wurden, sollen hier einige weitere Einstellungen und Erwartungen der Eltern bezüglich des Schulbesuchs dargestellt werden. Auch hier wieder antworteten in den meisten Fällen die Mütter. Um einen Hinweis auf den Stellenwert der Opportunitätskosten zu erhalten, wurden die Eltern zu einer potentiellen Beschäftigung der Schulkinder und zu dem Empfinden ihrer Abwesenheit während der Schulzeit befragt. Außerdem werden einige Daten zur Bewertung der Schule allgemein dargestellt.

\subsection{Einstellung zu einer Beschäftigung der Kinder}

Um den Stellenwert der Opportunitätskosten durch entgangene Einkommen zu untersuchen, wurden die Eltern nach ihrer Reaktion auf eine hypothetische Beschäftigung für jedes ihrer Kinder gefragt. Durch das Angebot einer bezahlten Arbeit sollten den Eltern die Opportunitätskosten des Schulbesuchs deutlich vor Augen geführt werden. Auch wenn sie bisher die Opportunitätskosten nicht bewusst in ihr Kalkül aufgenommen hatten, wird durch die Fragestellung deutlich, welche Einkommen den Kindern durch den Schulbesuch entgehen. Zuerst sollten sie entscheiden, ob das Kind eine angebotene bezahlte Arbeit annehmen darf, wenn es diese anstelle des Schulbesuchs ausführen müsste. Wie viele der Haushalte kategorisch ablehnend geantwortet haben, ist in der folgenden Tabelle zusammengefasst.

Tabelle 12.1: Bezahlte Arbeit anstelle des Schulbesuchs

\begin{tabular}{l|c|cccc}
\hline Das Kind soll auf keinen Fall arbeiten, & Stichpr. & \multicolumn{4}{|c}{ Bevölkerungsschicht } \\
sondern weiter zur Schule gehen! & gesamt & A & B & C & D \\
\hline gesamt & $98 \%$ & $98 \%$ & $100 \%$ & $97 \%$ & $97 \%$ \\
\hline$<12$ Jahre & $99 \%$ & $99 \%$ & $100 \%$ & $98 \%$ & $100 \%$ \\
$>$ 11 Jahre & $97 \%$ & $98 \%$ & $100 \%$ & $96 \%$ & $95 \%$ \\
\hline Jungen & $98 \%$ & $99 \%$ & $100 \%$ & $97 \%$ & $98 \%$ \\
Mädchen & $98 \%$ & $98 \%$ & $100 \%$ & $97 \%$ & $97 \%$ \\
\hline
\end{tabular}

Obwohl der Schulbesuch nicht als rentabel angesehen wird, soll kaum ein Kind die Basisausbildung für eine bezahlte Arbeit verlassen. Nur aus den beiden untersten Schichten bekämen 4\%-5\% der älteren Kinder die Erlaubnis, den Job anzunehmen. Eine abgebrochene Basisausbildung scheint für die Eltern keinen Wert zu haben, auch wenn den Kindern eine bezahlte Arbeit angeboten wird. Wie schon die Ertragserwartungen vermuten ließen, wird die Basisausbildung offensichtlich nicht als „Türöffner" für den Arbeitsmarkt betrachtet, sondern als 
Zugangsvoraussetzung für weitere Bildung, was einen erfolgreichen Abschluss der Basisausbildung voraussetzt.

Würde die Bildungsentscheidung auf einem ökonomischen Kalkül beruhen, müsste es ein Mindestgehalt geben, das die Opportunitätskosten so ansteigen lässt, dass ein Schulabbruch rentabel würde. Obwohl die Eltern die Möglichkeit hatten, ein solches Gehalt als Voraussetzung anzugeben, ergab sich die oben dargestellte Verteilung der kategorischen Ablehnungen. Entweder die Bildungsentscheidung beruhte nicht auf einem Investitionskalkül, oder das notwendige Einkommen müsste so hoch ausfallen, dass diese Alternative nicht als relevant betrachtet wurde. Die obigen Ausführungen zu den Einkommenserwartungen der Eltern zeigten bereits, dass die erwarteten Einkommenssteigerungen durch die Primaria und die Sekundaria nicht besonders hoch angenommen werden. Im Hinblick auf ein ausreichendes Einkommen scheint sich eine abgebrochene Basisausbildung demnach nicht deutlich von der Alternative „keiner Ausbildung“ zu unterscheiden. Ein Abbruch der Ausbildung kommt nicht in Frage, weil die bereits getätigten Investitionen verloren wären, ohne eine entsprechende Kompensation zu versprechen.

Die gleiche Fragestellung wurde bezüglich einer Tätigkeit gestellt, die die Kinder neben dem Schulbesuch ausüben können. Auch hier gibt die Tabelle die Anzahl der kategorischen Ablehnungen an.

Tabelle 12.2: Bezahlte Arbeit neben dem Schulbesuchs

\begin{tabular}{l|c|cccc}
\hline Das Kind soll auf keinen Fall arbeiten, & Stichpr. & \multicolumn{4}{|c}{ Bevölkerungsschicht } \\
sondern weiter zur Schule gehen! & gesamt & A & B & C & D \\
\hline gesamt & $65 \%$ & $82 \%$ & $72 \%$ & $62 \%$ & $59 \%$ \\
\hline$<$ 12 Jahre & $76 \%$ & $90 \%$ & $81 \%$ & $74 \%$ & $72 \%$ \\
> 11 Jahre & $52 \%$ & $73 \%$ & $62 \%$ & $48 \%$ & $45 \%$ \\
\hline Jungen & $63 \%$ & $80 \%$ & $70 \%$ & $59 \%$ & $58 \%$ \\
Mädchen & $67 \%$ & $85 \%$ & $76 \%$ & $65 \%$ & $61 \%$ \\
\hline
\end{tabular}

Wenn die Nebentätigkeit nicht zum Abbruch der Schule führt, lehnen die Eltern die Tätigkeit noch für 65\% ihrer Kinder ab. Die Möglichkeit, neben der Schule Geld zu verdienen, senkt die Opportunitätskosten des Schulbesuchs. Auch hier zeigt sich, dass die Eltern einem Job vor Abschluss der Ausbildung kein großes Einkommenspotential zutrauen. Vielmehr scheinen sie zu fürchten, dass die Nebentätigkeit den erfolgreichen Abschluss der Basisausbildung gefährden könnte.

Trotzdem zeigen die Daten, dass die Opportunitätskosten vor allem in den unteren Schichten Relevanz zu haben scheinen, da hier ein deutlich höherer Anteil der Kinder den Job annehmen dürften/sollten. Auch die Annahme, dass die Opportunitätskosten mit dem Alter steigen, wird hier bestätigt. In der untersten Schicht wird nur noch $45 \%$ der älteren Kinder ein Nebenjob untersagt. Obwohl 
auch in dieser Schicht das Einkommenspotential der Schüler nicht sehr hoch angenommen wird, spielen die Opportunitätskosten eine so große Rolle, dass sie durch die Nebentätigkeit der Kinder gesenkt werden sollen.

\subsection{Bewertung der Abwesenheit der Kinder}

Ebenfalls auf die Opportunitätskosten sollte die Frage nach der Bewertung der Abwesenheit der Kinder hindeuten. ${ }^{328}$ Auch hier zeigt sich, dass die klassischen Opportunitätskosten durch entgangene Einkommen und fehlende Mithilfe im Haushalt in den Empfindungen der Eltern kaum Relevanz haben. Insgesamt erklärten nur 2\% der Haushalte der obersten Schicht und 6\% der Haushalte der untersten Schicht diese Empfindungen als vorrangig. Sie werden häufiger für die älteren Kinder genannt und vor allem die Mädchen fehlen bei der Hausarbeit.

Tabelle 12.3: Empfindungen bei Abwesenheit der Kinder

\begin{tabular}{l|c|rrrr}
\hline Wie nehmen Sie die Abwesenheit der Kinder & Stichpr. & \multicolumn{4}{|c}{ Bevölkerungsschicht } \\
während des Schulbesuchs wahr? & gesamt & A & B & C & D \\
\hline negativ, weil sie nicht zum Eink. beitragen & $2 \%$ & $2 \%$ & $2 \%$ & $2 \%$ & $3 \%$ \\
negativ, weil sie nicht zur Hausarbeit beitragen & $1 \%$ & $0 \%$ & $0 \%$ & $1 \%$ & $3 \%$ \\
positiv, weil sie nicht zu beaufsichtigen sind & $24 \%$ & $30 \%$ & $29 \%$ & $17 \%$ & $25 \%$ \\
negativ, weil ich sie vermisse & $47 \%$ & $30 \%$ & $39 \%$ & $49 \%$ & $52 \%$ \\
weder positiv noch negativ & $26 \%$ & $39 \%$ & $31 \%$ & $32 \%$ & $17 \%$ \\
\hline
\end{tabular}

In den unteren Schichten stehen Empfindungen wie Traurigkeit und Fremdheit, weil die Eltern ihre Kinder zuhause vermissen, an erster Stelle. Diese Empfindungen können als nichtmonetäre Kosten der Ausbildung bezeichnet werden. Sie sind etwas ausgeprägter für die kleineren Kinder. Die meisten Familien der obersten Schicht empfinden die Abwesenheit der Kinder weder negativ noch positiv.

Positive Empfindungen, weil die Schule die Aufsichtsfunktion für die Kinder übernimmt, wurden ebenfalls von fast $25 \%$ der Haushalte genannt. Hier handelt es sich um positive Externalitäten des Schulbesuchs. Sie werden von den Haushalten der obersten Schicht gleichauf mit dem Vermissen der Kinder genannt und verlieren mit sinkendem sozioökonomischen Niveau der Familien an Relevanz.

328 Die zu wählenden Antworten zu den Opportunitätskoten waren vorgegeben, der Katalog wurde aber während einer Pilotbefragung durch oft genannte weitere Empfindungen erweitert. 


\subsection{Bewertung der Schule}

Als letzter Punkt diese Abschnittes sollen einige Indikatoren für die Wertschätzung der Eltern für die Schule dargestellt werden. Sie halten die Basisausbildung für notwendig, da sich die meisten Eltern eine höhere Ausbildung ihrer Kinder wünschen. Und sie halten die Basisausbildung für sich genommen nicht für rentabel. Hier soll nun untersucht werden, was sie darüber hinaus von der Schule halten.

Zunächst wurde gefragt, wie sie den Lehrerberuf in der Gesellschaft einschätzen. Die Wertschätzung der Lehrer spiegelt die Wertschätzung der Ausbildungseinrichtung wider. In Peru stellt die geringe Akzeptanz der Lehrer durch die Eltern ein großes Problem dar, das sich negativ auf die Wahrnehmung der Schulen auswirkt.

Tabelle 12.4: Wertschätzung der Lehrer in der Gesellschaft

\begin{tabular}{l|c|rrrr}
\hline Glauben Sie, dass der Beruf des Lehrers & Stichpr. & \multicolumn{4}{|c}{ Bevölkerungsschicht } \\
in der Gesellschaft ... & gesamt & A & B & C & D \\
\hline ...sehr respektiert ist & $4 \%$ & $4 \%$ & $2 \%$ & $4 \%$ & $5 \%$ \\
... respektiert ist & $35 \%$ & $30 \%$ & $22 \%$ & $40 \%$ & $40 \%$ \\
... wenig respektiert ist & $52 \%$ & $52 \%$ & $63 \%$ & $46 \%$ & $50 \%$ \\
...überhaupt nicht respektiert ist & $9 \%$ & $13 \%$ & $12 \%$ & $10 \%$ & $6 \%$ \\
\hline
\end{tabular}

Eine deutliche Mehrheit in allen Schichten hält den Lehrer für wenig oder gar nicht respektiert in der Gesellschaft. In den unteren Schichten fällt das Urteil etwas positiver aus. Die Kinder haben also eine Berufsgruppe zum Vorbild, deren Anerkennung in der Gesellschaft relativ niedrig ausfällt.

Diese geringe Wertschätzung der Lehrer kann mit der relativ niedrigen Position der Einkommen der Lehrer im Vergleich mit den Einkommen in anderen Berufszweigen zusammenhängen. Die relative Position der Lehrer sank um 30\% zwischen 1986 und 1992. Sie verbesserte sich nach 1990 zwar wieder und überholte sogar die Einkommen des öffentlichen Sektors. Dennoch könnten die besseren Einkommenschancen im privaten Sektor zu einer Abwanderung der kompetenteren Lehrer in diesen Sektor geführt haben. Auch bieten die niedrigen Entlohnungsunterschiede in den verschiedenen Bildungsstufen keinen großen Anreiz für die Lehrer sich fortzubilden. Außerdem weisen nur $62 \%$ der peruanischen Lehrer einen pädagogischen Titel auf. Die restlichen 38\% unqualifizierten Lehrer nehmen einen noch niedrigeren Status ein als ihre pädagogisch ausgebildeten Kollegen. 329 
Als weiteren Hinweis auf die Wertschätzung der Ausbildung wurde gefragt, ob die Schule die Kinder verändert. Dazu wurden den Eltern zwei Thesen vorgegeben, denen sie zustimmen oder die sie ablehnen sollten. Die Tabelle fasst die Ergebnisse zusammen.

Tabelle 12.5: Auswirkungen der Ausbildung auf die Kinder

\begin{tabular}{|c|c|c|c|c|c|}
\hline \multirow[t]{2}{*}{ Kinder, die in die Schule gehen, werden... } & \multirow{2}{*}{$\begin{array}{l}\text { Stichpr. } \\
\text { gesamt }\end{array}$} & \multicolumn{4}{|c|}{ Bevölkerungsschicht } \\
\hline & & A & B & C & $D$ \\
\hline \multicolumn{6}{|l|}{...eingebildet und helfen zuhause nicht mehr } \\
\hline ich stimme zu & $22 \%$ & $13 \%$ & $18 \%$ & $23 \%$ & $26 \%$ \\
\hline ich stimme nicht zu & $75 \%$ & $85 \%$ & $79 \%$ & $76 \%$ & $69 \%$ \\
\hline \multicolumn{6}{|l|}{... aufsässig und verursachen zuhause Probleme } \\
\hline ich stimme zu & $18 \%$ & $9 \%$ & $11 \%$ & $22 \%$ & $21 \%$ \\
\hline ich stimme nicht zu & $80 \%$ & $91 \%$ & $86 \%$ & $76 \%$ & $77 \%$ \\
\hline
\end{tabular}

Obwohl die Thesen provokativ eine negative Verhaltensänderung der Kinder darstellen, beurteilten insgesamt rund 20\% diese Aussagen als richtig.

Als erste These wurde genannt, dass Kinder, die in die Schule gehen, eingebildet werden und nicht mehr bei der Hausarbeit mithelfen wollen. Dieser These stimmten zwar nur 13\% der Haushalte der obersten Schicht aber immerhin 26\% der Haushalte der untersten Schicht zu. Über ein Viertel der Haushalte der untersten Schicht erwarten also derartig negative Folgen eines Schulbesuchs ihrer Kinder.

Der These, dass die Kinder aufsässig werden und zuhause Probleme verursachen, stimmen 9\% der Haushalte der obersten Schicht und 21\% der untersten Schicht zu. In den unteren Schichten wird der Schule also größeres Potential zugetraut, die Kinder zur Auflehnung gegen ihre Eltern zu veranlassen.

Als nächstes wurde gefragt, welche Aufgaben - über die Wissensvermittlung hinaus - die Schule noch ausüben sollte. Die Eltern sollten angeben, für wie wichtig sie die folgenden Ziele halten. 330

330 Dabei sollten sie den einzelnen Zielen Bewertungen von „sehr wichtig“ bis „nicht wichtig“ zuordnen. Diese Bewertungen wurden in Rangordnungen umgewandelt. 
Die Tabelle zeigt die Rangordnung der Ziele für die einzelnen Schichten.

Tabelle 12.6: Aufgaben der Schule

\begin{tabular}{|c|c|c|c|c|c|}
\hline \multirow[t]{2}{*}{ Die Schule sollte... } & \multirow{2}{*}{$\begin{array}{l}\text { Stichpr. } \\
\text { gesamt }\end{array}$} & \multicolumn{4}{|c|}{ Bevölkerungsschicht } \\
\hline & & A & B & C & D \\
\hline ...den Wunsch nach weiterer Ausbildung steigern & 1 & 3 & 1 & 2 & 1 \\
\hline ...das Selbstbewusstsein stärken & 2 & 1 & 2 & 3 & 2 \\
\hline ...lehren selbständig zu denken & 3 & 2 & 4 & 1 & 4 \\
\hline ...auf die Arbeit vorbereiten & 4 & 4 & 3 & 4 & 4 \\
\hline ...lehren im Team zu arbeiten & 5 & 4 & 5 & 6 & 6 \\
\hline ...lehren zu gehorchen & 6 & 6 & 6 & 5 & 3 \\
\hline
\end{tabular}

Tatsächlich rangiert das Ziel, den Wunsch nach weiterer Ausbildung zu generieren, insgesamt an oberster Stelle. Nur in der obersten Schicht fällt diese Aufgabe der Schule auf den dritten Platz. Ein Grund dafür könnte sein, dass in dieser Schicht eine Fortführung der schulischen Laufbahn nach der Basisausbildung als so selbstverständlich angenommen wird, dass dieser Wunsch nicht erst in der Schule vermittelt werden muss. ${ }^{331}$ Die Schule als Vorbereitung auf die Arbeit und auf Teamarbeit wird erst an vierter und fünfter Stelle genannt. Auch hier zeigt sich die oben schon vermutete Rolle der Basisausbildung als Zugangsvoraussetzung für weiterführende Bildung. Ihre Relevanz als Vorbereitung auf den Arbeitsmarkt ist gering.

Auf den Plätzen zwei und drei stehen die Stärkung des Selbstbewusstseins und die Fähigkeit selbständig zu denken. Hier spiegelt sich der Wunsch der Eltern wider, dass die Schule eine große Rolle spielen soll bei der Formung der Persönlichkeiten der Kinder. An letzter Stelle liegt die Aufgabe der Schule, den Kindern beizubringen, dass sie zu gehorchen haben. Diese Bewertung und die hohe Bewertung für die Steigerung des Selbstbewusstseins zeigen, dass die Kinder zu mehr Eigeninitiative erzogen werden sollen.

In den beiden unteren Schichten wird dem Selbstbewusstsein etwas weniger und dem Gehorsam etwas mehr Bedeutung beigemessen. Diese Bewertung passt zu den negativen Erfahrungen, die einige Eltern aus diesen Schichten mit ihren Kindern gemacht haben, seit diese zur Schule gehen.

331 Vgl. dazu die Ausführungen zu dem möglichen Einfluss von Normen und Traditionen in Abschnitt 4.2. 


\subsection{Zusammenfassung der sonstigen Einstellungen}

Einige Fragen zu einer hypothetischen Beschäftigung der Kinder haben gezeigt, dass den Opportunitätskosten in Form von entgangenen Einkommen bei der Basisausbildung nur geringe Relevanz zukommt. Der Einfluss der Opportunitätskosten ist wie erwartet in den unteren sozioökonomischen Schichten größer als in den obern Schichten.

Die Beurteilung der Schule ist als ambivalent zu bezeichnen. Obwohl die meisten Eltern den Beruf des Lehrer als nicht besonders akzeptiert bezeichnen und einige auch mit negativen Verhaltensänderungen durch den Schulbesuch der Kinder rechnen, erwarten sie von der Schule, dass sie neben ihrer Funktion als Voraussetzung für höhere Bildung auch an der Persönlichkeitsentwicklung der Kinder mitwirkt. Dabei kommt es den Eltern weniger auf Gehorsam als auch die Formung eigenständiger und selbstbewusster Persönlichkeiten an.

Auch die Erkenntnisse der Rentabilitätsuntersuchung wurde durch direkte Befragung unterstützt. Die Eltern wünschen sich von der Basisschule vor allem, dass sie den Wunsch nach weiterer Bildung schürt. Das Ziel, die Kinder auf den Arbeitsmarkt vorzubereiten, rangiert erst auf dem vierten von sechs Plätzen. 



\section{Teil: Zusammenfassung und Schlussfolgerungen}

\section{Zusammenfassung und grafische Darstellung des privaten Investitionskalküls}

Der theoretische Teil dieser Arbeit (Teil I) hat gezeigt, dass die private Ertragsrate ein wichtiges Element in der individuellen Bildungsnachfrage darstellen kann. Auch wenn die Haushalte keine exakten Ertragsraten errechnen, wie dies die ökonomische Theorie unterstellt, kann davon ausgegangen werden, dass sie eine Idee des Kosten-Nutzen-Verhältnisses der Bildungsinvestition haben. Diese Relation wird ihre Bildungsentscheidung beeinflussen. Die bildungsökonomische Theorie geht davon aus, dass die Individuen anhand der unterschiedlichen Rentabilitäten verschiedener Bildungsstufen ihre Investitionsentscheidung anhand eines Optimierungskalküls treffen. Die grafische Darstellung dieses Optimierungskalküls wurde ebenfalls aufgezeigt. Dabei wird das Bildungsniveau gewählt, bei dem der Grenzertrag der Ausbildung dem Grenzertrag der Investitionskosten der Ausbildung bzw. dem Grenzertrag einer alternativen Investition entsprechen.

In der bildungsökonomischen Theorie sind es vor allem die Kosten und die Nutzenfaktoren, die den Wert der Ausbildung bestimmen. Sie bestimmen die Lage und den Verlauf der Ertragsratenkurve und damit das optimale Bildungsniveau. Allerdings zeigte die Auswertung zahlreicher empirischer Studien (Teil II), dass den direkten Kosten kein großer Einfluss auf die Bildungsentscheidung nachgewiesen werden konnte. Einige Indikatoren weisen aber darauf hin, dass die Opportunitätskosten des Schulbesuchs eine deutlichere Rolle spielen. Die Rolle der Nutzenerwartungen auf die Bildungsentscheidung wurde hingegen in der empirischen Literatur vernachlässigt. Für Entwicklungsländer finden sich kaum Untersuchungen, die die Einkommenserwartungen der Individuen behandeln. Dementsprechend gibt es auch keine Untersuchungen über deren Realitätsgehalt. Einige wenige Studien aus den USA zeigten allerdings, dass Studenten höherer Bildungsstufen eine durchaus realistische Einschätzung bezüglich ihrer zukünftigen Einkommen haben. Der Einfluss dieser Erwartungen auf die Bildungsnachfrage ist zwar anzunehmen, empirisch aber kaum belegt.

Dahingegen wurde in der empirischen Literatur ausführlich der Zusammenhang zwischen individuellen und familiären Charakteristika und der Bildungsnachfrage untersucht. Sie zeigten einen schwachen positiven Einfluss des Einkommensniveaus und einen deutlichen Einfluss des Bildungsniveaus der Eltern auf die Ausbildung der Kinder. Dahinter steckt die Vermutung, dass vor allem die 
Präferenzen und Einstellungen der Eltern die Bildungsnachfrage für ihre Kinder bestimmen. Hier bedarf es allerdings noch weiterer Forschung.

Diese Lücke konnte mit der Auswertung einer eigenen Haushaltsbefragung zur Erwartungen und Einstellungen der Eltern bezüglich der Ausbildung ihrer Kinder (Teil III) ein Stück weit geschlossen werden.

Zunächst konnte gezeigt werden, dass sich die Haushalte der verschiedenen sozioökonomischen Schichten gerade in den die Bildungsnachfrage beeinflussenden Charakteristika deutlich voneinander unterscheiden. Die Ausbildungssituation der Kinder bei der Basisausbildung unterscheidet sich vor allem durch die Art der besuchten Schule und durch ihre Leistungen. Die Bildungsteilnahme an der Basisausbildung ist hingegen über alle Schichten hinweg als umfassend zu betrachten.

Die Befragung zu den Ertragserwartungen lieferte vor allem zwei interessante Ergebnisse: Erstens konnte ermittelt werden, dass die Familien der Basisausbildung keinen ökonomischen Wert beimessen. Sie betrachten die Ausbildung eher als notwendige Voraussetzung für höhere Bildung. Diese Erkenntnis hat immense Auswirkungen auf die Wirksamkeit von Maßnahmen der Bildungspolitik. 332

Zweitens zeigt die Untersuchung, dass Bevölkerungsgruppen unterschiedlicher Schichten unterschiedliche Erwartungen bezüglich der zukünftigen Einkommen ihrer Kinder bilden. Aus diesen Erwartungen wird der Wert der Ausbildung bemessen und die Bildungsnachfrage bestimmt. Die unterschiedlichen Erwartungen an die Schule und deren Bewertung können einen Hinweis darauf geben, warum die Kinder ärmerer Haushalte schlechter in der Schule abschneiden als ihre Mitschüler aus reicheren Familien. ${ }^{333}$

Im folgenden Absatz werden die in der eigenen Untersuchung ermittelten Erkenntnisse bei der grafische Darstellung des privaten Investitionskalküls berücksichtigt. Anhand dieser Darstellung werden repräsentative Kurvenverläufe für arme und reiche Haushalte ermittelt und Schlussfolgerungen für ihre Bildungswünsche gezogen, die sich wiederum auf ihre Nachfrage nach Ausbildung auswirken können.

332 Vgl. dazu die Zusammenfassung der Gemeinsamkeiten aller Bevölkerungsschichten bezüglich der Rentabilitätserwartungen der Basisausbildung in Abschnitt6.3.4.1.3.

333 Wenn zum Beispiel die Schule von den Eltern als lästige Pflichtveranstaltung betrachtet wird, wird sich diese Einstellung auf die Motivation der Kinder auswirken. 
Die im dritten Teil gewonnenen Erkenntnisse sollen anhand der theoretischen Argumente des ersten Teils, insbesondere unter Verwendung der grafischen Darstellung, interpretiert werden. Dazu sollen die Ertragsraten der Basisausbildung untersucht werden. Die Rentabilitätserwartungen der Eltern zeigten, dass die Ertragsraten der Basisausbildung in fast allen Familien unter dem Vergleichszins lagen. Die der Primarausbildung lag deutlich darunter und die der Sekundarbildung lag (vor allem für die Haushalte der oberen Schicht) schon dichter daran.

Würde für beide Investitionen der gleiche Vergleichszins (also ein horizontaler Verlauf der Finanzierungskostenkurve) angenommen, müsste die Ertragsrate der Primarausbildung weiter unter dieser Kurve liegen als die der Sekundarausbildung. Damit ist von einem steigenden Verlauf der Ertragsratenkurve für die Basisausbildung auszugehen.

Diese Entwicklung wird sogar noch verstärkt, wenn die Annahme eines konstanten Zinses aufgehoben wird. Steigt die Finanzierungskostenkurve an, muss die Ertragsratenkurve stärker steigen, um für die Basisausbildung unterhalb der Finanzierungskurve zu liegen. In der grafischen Darstellung wird dieser Zusammenhang deutlich.

\section{Grafik 13.1: Kapitalkosten und Ertragsraten für die Basisausbildung}

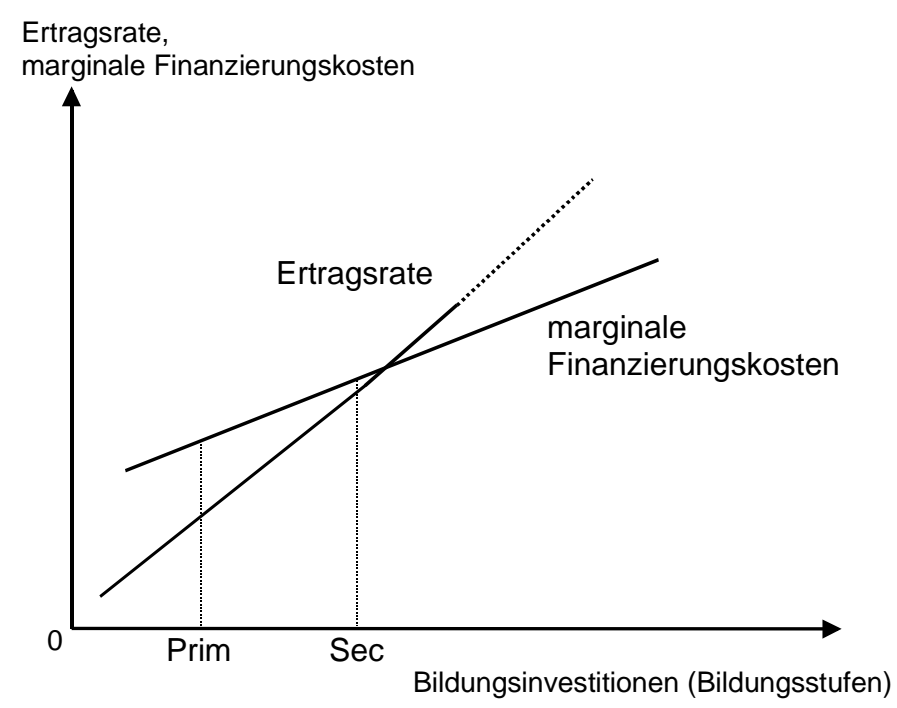

Bei höherem Kapitalbedarf muss mit steigenden Zinsen gerechnet werden. Der Sekundarbildung muss dann ein höherer Vergleichszins gegenübergestellt werden als der Primarbildung. Die von den Eltern erwarteten Ertragsraten liegen dann sogar noch deutlicher auseinander. Für beide Stufen der Basisausbildung liegt der Marktzins über der Ertragsrate. Wenn die Bildungsinvestition am Kapitalmarkt finanziert werden müsste, wäre der zu zahlende Zins höher als die Ertragsrate. Auch eine Anlage am Kapitalmarkt verspräche eine höhere Verzinsung. Die Individuen würden nicht in die Basisausbildung investieren. 
Wird nun angenommen, dass dieser für die Basisausbildung ermittelte Verlauf der Ertragsraten sich fortsetzt, dann würde die Ertragsratenfunktion die Zinsfunktion bei einem Bildungsniveau kurz über der Basisausbildung schneiden. Ab diesem Schnittpunkt würde mit jedem zusätzlichen Bildungsjahr die Investition rentabler. Ein eindeutiges optimales Bildungsniveau könnte nicht ermittelt werden. Mit jeder Ausweitung der Ausbildung um ein Jahr könnte ein höherer Zinsgewinn erreicht werden, da die Zinsdiskrepanz immer weiter zugunsten der Ausbildung steigt.

Bei einem derartigen Verlauf der Kurven müsste in der Realität zu beobachten sein, dass nahezu alle Individuen unendlich viel Bildung nachfragen. Dies ist aber vor allem in Entwicklungsländern nicht zu beobachten. Der Verlauf ist allerdings auch nicht als realistisch zu bezeichnen. Die folgenden Überlegungen werden zeigen, warum ab einem Niveau der höheren Bildung wieder mit sinkenden Ertragsraten zu rechnen ist.

Zunächst lassen die Untersuchungsergebnisse aus Teil III darauf schließen, dass die höhere Bildung tatsächlich rentabel wird, da sonst die große Nachfrage nach weiterführender Ausbildung nicht zu erklären wäre. Die Ertragsrate muss also tatsächlich den Finanzierungszins überschreiten. Ein deutlicher Hinweis auf diese Entwicklung ist der enorme Einkommensanstieg, der mit einer Ausbildung über die Basisausbildung hinaus erwartet wird.

Die Haushaltsbefragung hat außerdem gezeigt, dass die Basisausbildung in Lima nicht in Konkurrenz zu einer Beschäftigung am Arbeitsmarkt steht. Kaum ein Schüler hat die Schule verlassen, um einer bezahlten Tätigkeit nachzugehen. Auch geht nur ein sehr kleiner Teil (4\%) der Schüler einer Nebentätigkeit nach. Die Befragung der Eltern zu einer hypothetischen Beschäftigung der Kinder hat gezeigt, dass dieser Alternative im Basisschulalter keine Relevanz eingeräumt wird. Die Einkommenserwartungen zeigten, dass die Einkommen für Abbrecher der Basisausbildung so niedrig sind, dass sie nicht als Alternative zur Ausbildung betrachtet werden. Die Opportunitätskosten durch entgangene Einkommen werden also während der Basisausbildung als sehr niedrig angenommen oder gar nicht berücksichtigt, da die Alternative Schulabbruch oder die Ausübung einer Nebentätigkeit den erfolgreichen Abschluss der Basisausbildung und damit die Investition insgesamt gefährden könnte. Das Ziel der Investition ist offensichtlich die Erreichung hoher Einkommen durch die höhere Ausbildung.

Diese Zusammenhänge ändern sich grundlegend mit dem Abschluss eines höheren Bildungsniveaus. Die Daten zeigten, dass ab diesem Bildungsniveau mit hohen Einkommenssteigerungen gerechnet wird. Jetzt werden die möglichen Einkommen also so hoch bewertet, dass sich ein Verzicht auf Weiterbildung 
lohnen könnte. Die hohen Einkommenserwartungen ab einer bestimmten Stufe der höheren Ausbildung haben gleichzeitig hohe erwartete Opportunitätskosten durch entgangene Einnahmen zur Folge, wenn die Ausbildung über dieses Niveau fortgesetzt würde. Die Opportunitätskosten durch entgangene Einkommen stellen nun einen erheblichen Faktor in der Investitionsentscheidung dar, und führen dazu, dass die Ertragsraten ab einem bestimmten Niveau der höheren Bildung wieder sinken.

Die Argumentation folgt hier den Überlegungen zum Verlauf der Ertragsratenkurve in der bildungsökonomischen Theorie. ${ }^{334}$ Erstens wird mit jedem zusätzlichen Schuljahr ab diesem Bildungsniveau auf ein Jahr mit diesem hohen Verdienst verzichtet. Das heißt, die Opportunitätskosten durch entgangene Einkommen sind hoch. Außerdem verkürzt sich der Verdienstzeitraum um die Jahre der zusätzlichen Ausbildung, da das Lebensalter begrenzt ist. Auch diese Verkürzung fällt jetzt stärker ins Gewicht, weil die angenommenen Verdienste sehr hoch sind.

Damit lässt sich erklären, warum die Ertragsraten während der Baisausbildung ansteigen, und erst ab einem bestimmten Niveau der höheren Bildung wieder durch die Opportunitätskosten gedämpft werden. Außerdem ist natürlich nicht davon auszugehen, dass die erwarteten Einkommen mit jeder zusätzlichen Stufe der höheren Bildung im gleichen Maße gesteigert wird. Ein möglicher Verlauf der Ertragsratenfunktion wird in der folgenden Grafik dargestellt.

\section{Grafik 13.2: Verlaufsänderung der Ertragsraten für die höhere Bildung}

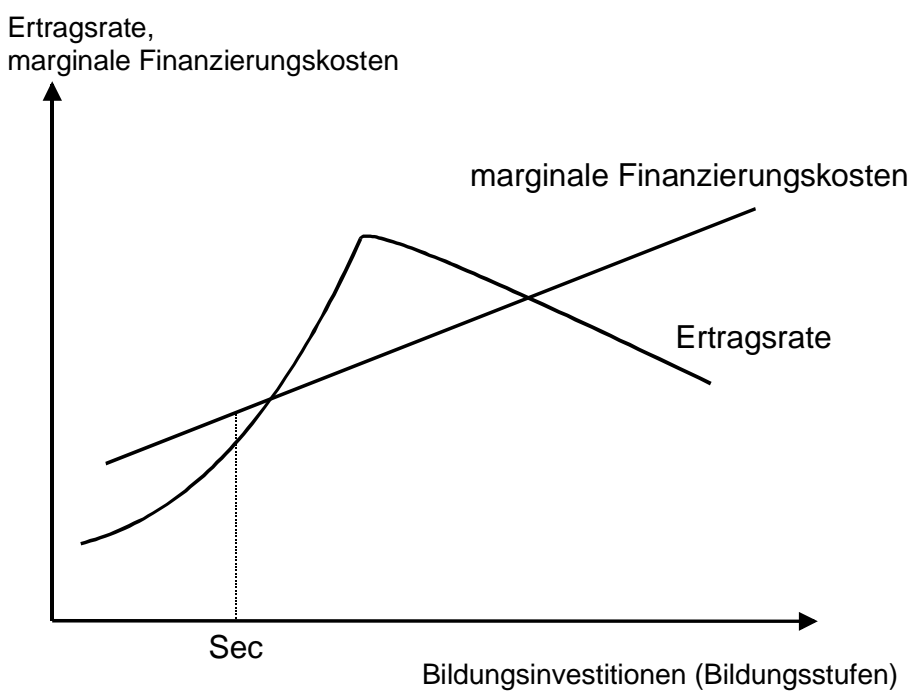

Aufgrund der Untersuchungen ist also zu vermuten, dass der Verlauf der Ertragsratenfunktion ab einem bestimmten Niveau der höheren Bildung genau den Annahmen der im dritten Kapitel dargestellten Theorie folgt. Nur die Integ-

334 Vgl. die Ausführungen zu den Gründen für den Verlauf der Kurven in Kapitel 3.1.1. 
ration der Basisausbildung in das Nutzenkalkül scheint anderen Gesetzen zu folgen.

Der Basisausbildung für sich genommen wird kein ökonomischen Wert zugewiesen. Für sie erfolgt separat kein Investitionskalkül. Sie wird vielmehr als Zugangsvoraussetzung für die weitere Bildung betrachtet und ist mit absoluten Verlusten verbunden. Das Kalkül beginnt erst bei der höheren Bildung, weil erst hier ein Ausscheiden aus dem Bildungssystem zu einer relevanten Alternative wird. ${ }^{335}$ Die erste Ertragsrate wird daher für den ersten möglichen Abschluss einer höheren Bildung berechnet. Sie enthält alle bis dahin angefallenen Kosten und Nutzen. So kann sichergestellt werden, dass die Kosten der Basisausbildung in das Kalkül eingehen und die Bildungsstufe gewählt wird, bei der das Gesamtkalkül optimal wird. Eine grafische Darstellung würde dann erst bei dem Bildungsniveau beginnen, bei dem ein Ausstieg aus der Ausbildung eine relevante Alternative zum weiteren Schulbesuch wäre, die Ertragsratenfunktion also wieder sinkt. Der Kurvenverlauf hat ab diesem Punkt wieder den aus der Bildungsökonomie bekannten Verlauf. Dieser Verlauf wird in der folgenden Grafik noch einmal dargestellt.

\section{Grafik 13.3: Kurvenverläufe für die höhere Bildung}

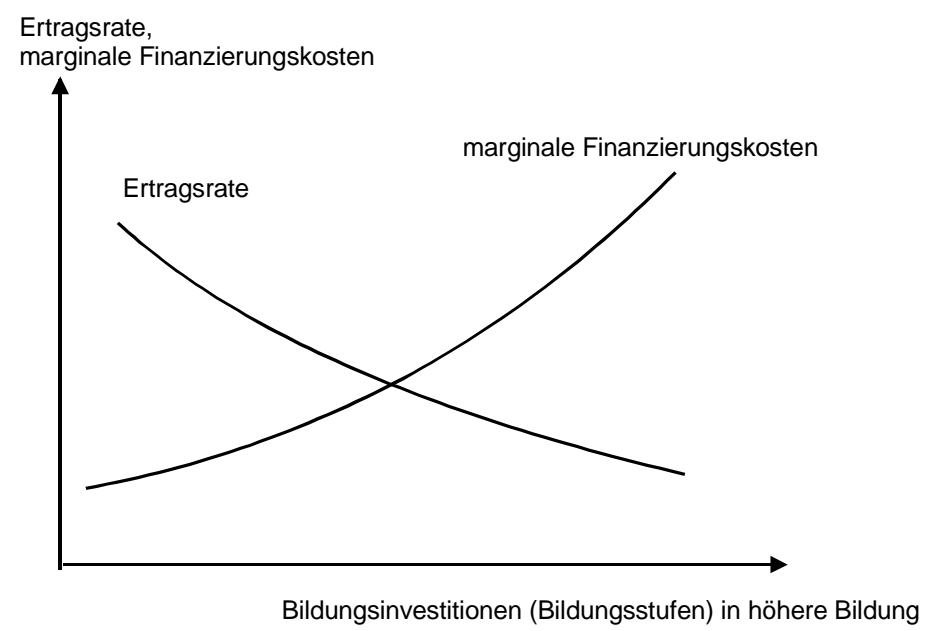

Für jeden möglichen Abschluss der höheren Bildung werden die Zinssätze verglichen und das Bildungsniveau gewählt, bei dem die Ertragsrate dem Finanzierungszins entspricht.

Mit diesen Erkenntnissen kann nun die Bildungsentscheidung der Haushalte aus verschiedenen sozioökonomischen Schichten untersucht werden. Dies erfolgt im nächsten Kapitel.

335 Die Interpretation erfolgt analog zu den Ertragsraten in der Bildungsliteratur. Auch hier werden keine Ertragsraten für einzelne Jahre ermittelt, da davon ausgegangen wird, dass die Entscheidungen immer für die nächste Stufe getroffen wird. Ein Ausstieg nach einzelnen Jahren vor Abschluss der Bildungsstufe wird als irrelevante Alternative betrachtet. 


\section{Auswirkungen von schichtenspezifischen Unterschieden in den Ertragserwartungen für höhere Bildung}

Anhand der oben dargestellten Grafiken soll nun untersucht werden, wie sich die Investitionskalküle von armen und reichen Haushalten bezüglich der höheren Bildung unterscheiden. Eine derartige Untersuchung ist daher sinnvoll, weil dadurch Unterschiede in der Bildungsnachfrage ab diesem Bildungsniveau erklärt werden können. Dazu werden nur zwei Schichten zur Analyse herangezogen, die vereinfachend als arm und reich klassifiziert werden sollen.

Hier sei nochmals darauf hingewiesen, dass die vorangegangenen Erkenntnisse darauf hinweisen, dass das Investitionskalkül erst bei der höheren Bildung beginnt. Ein auf dem Investitionskalkül basierender Unterschied in der Bildungsnachfrage der beiden Bevölkerungsgruppen kann daher erst für die höhere Bildung erfolgen. Die Ausführungen im dritten Teil haben außerdem gezeigt, dass die Teilnahme an der Basisausbildung über die Schichten hinweg nahezu umfassend ist. Auch der Wunsch nach höherer Bildung ist nahezu allen Familien gemein. Allerdings ist dieser Wunsch schon daher zu erklären, dass bei der Basisausbildung mit Verlusten gerechnet wird, die erst durch die höhere Bildung wieder ausgeglichen werden können.

Die Haushaltsbefragung zeigte, dass vor allem in den Erwartungen über die zukünftigen Einkommen deutliche Unterschiede zwischen den Schichten bestehen. Wird davon ausgegangen, dass sich diese Erwartungen in einem Investitionskalkül niederschlagen, kann mit diesen Informationen ein optimales Bildungsniveau für die beiden Schichten ermittelt werden. Allerdings wird die Analyse dadurch erschwert, dass in Lima ein Großteil der Kinder armer Familien öffentliche Schulen besuchen, während Kinder reicher Eltern in Privatschulen gehen. Die Aussagen zu den Einkommenserwartungen beziehen sich daher auf den Abschluss unterschiedlicher Schularten. Da diesen Schularten auch unterschiedliche Kosten zugrunde liegen, sind die Ergebnisse nicht ohne weiteres vergleichbar. Deshalb wird zunächst davon ausgegangen, dass alle Individuen das gleiche Schulsystem besuchen. Diese Annahme wird anschließend wieder aufgehoben. Dadurch kann gezeigt werden, dass die ermittelten Unterschiede in den optimalen Bildungsniveaus auch unter Berücksichtigung der unterschiedlichen Schularten weiterhin Bestand haben. 


\subsection{Unter der Annahme vergleichbarer Schulen}

Die Haushaltsbefragung zeigte, dass reiche Haushalte deutlich höhere Einkommenssteigerungen durch die Ausbildung ihrer Kinder erwarten als arme Haushalte. Allerdings zeigen die Auswertungen auch, dass die reichen Familien höhere Beträge in die Ausbildung ihrer Kinder investieren. Beide Gruppen gaben an, dass sie Verluste durch den Abschluss der Sekundarbildung erwarten. Das heißt, dass die Ertragsraten der Sekundarbildung unter dem Vergleichszins lagen. Die Ertragsrate der reichen Haushalte lag aber wesentlich dichter an diesem Zins als die der armen Haushalte, was inre Investition rentabler macht. Durch die wesentlich stärker steigenden erwarteten Einkommen nach der höhere Bildung bei dieser Gruppe, muss davon ausgegangen werden, dass der Schnittpunkt der beiden Funktionen bei den reichen eher erreicht ist und die Ertragsratenfunktion höher über die Zinsfunktion hinausschießt.

Eine eindeutige Aussage über die Lage der Ertragsraten der beiden Gruppen zueinander ist nicht so einfach möglich. Zwar muss angenommen werden, dass reiche Haushalte bessere Finanzierungssituationen aufweisen und damit bei jedem Investitionsbetrag niedrigere Zinsen zu zahlen haben als die armen Haushalte. ${ }^{336}$ Allerdings investieren sie auch mehr in jedes Bildungsniveau, was sie bei steigenden Zinssätzen höhere Zinsen zahlen lässt. Ob dieser „Zinseffekt" der höheren Investition die vorteilhafteren Finanzierungskonditionen nur schmälert oder sogar überkompensiert, kann nicht eindeutig geklärt werden. Damit ist nicht sicher, welche Einkommensgruppe bei gegebenem Bildungsniveau einen niedrigeren Zins aufweist. Damit kann auch keine Aussage über die relative Lage der Ertragsraten der beiden Gruppen zueinander gemacht werden.

Um diese Problem zu umgehen, wird zunächst davon ausgegangen, dass beide Einkommensgruppen ihre Kinder auf vergleichbare Schulen schicken und damit ungefähr die gleichen direkten Kosten zu tragen haben. Damit wird einer der beiden Zinseffekte ausgegrenzt.

336 Eine detaillierte Analyse der Finanzierungskostenkurve erfolgt für die beiden Bevölkerungsgruppen im nächsten Abschnitt. 


\subsubsection{Typische Finanzierungskostenkurven}

Zunächst soll eine detaillierte Beschreibung erfolgen, wie der Verlauf der Finanzierungskostenkurve zustande kommt und wodurch ihre Lage beeinflusst wird. Anschließend kann gezeigt werden, welche Verläufe für die beiden Bevölkerungsgruppen angenommen werden können und wie diese zueinander liegen.

Bei der Betrachtung der Finanzierungssituation der Haushalte, geht es um die Kosten der Bereitstellung der Mittel, die für die Ausbildung der Kinder aufgebracht werden müssen. Das Schulgeld selber spielt nur insofern eine Rolle, als dass es die Höhe der Investition festlegt. Unter der Annahme vergleichbarer Schulen wird zunächst angenommen, dass für ein bestimmtes Bildungsniveau jeder Haushalt das gleiche Schulgeld aufzubringen hat. Trotzdem kann es zu Kostenunterschieden kommen, wenn die Haushalte unterschiedlich hohe Zinsen für die Bereitstellung dieser Mittel zahlen müssen. Der Zusammenhang wird anhand der folgenden Grafik erklärt.

\section{Grafik 14.1: Individuelle Finanzierungskostenkurve}

marginale Finanzierungskosten

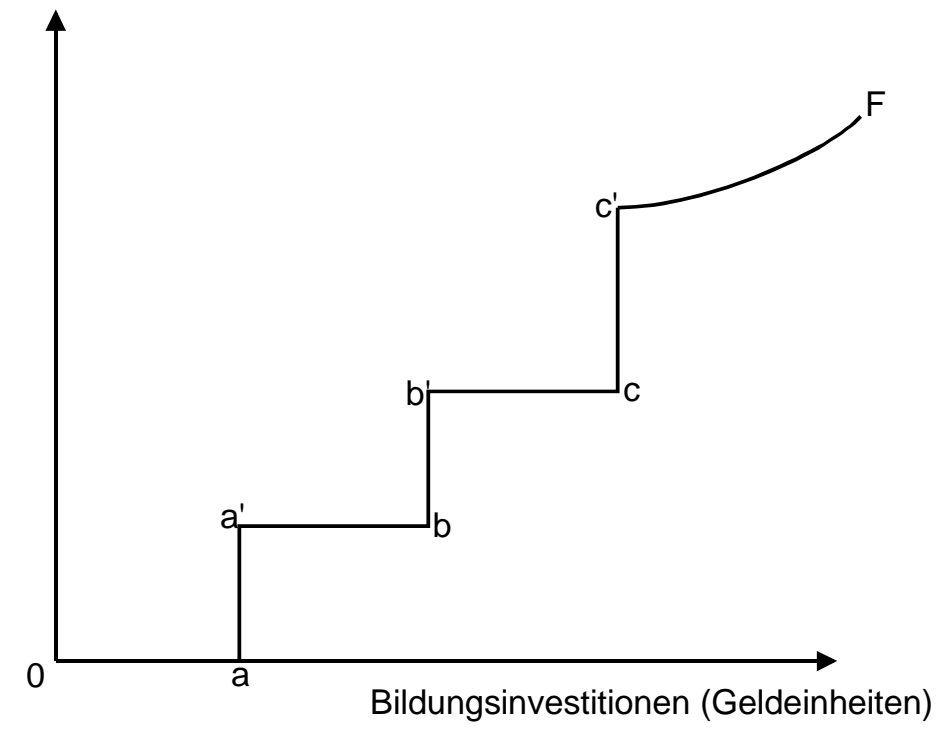

Quelle: Becker (1983), S. 98.

Entgegen der bisherigen Darstellungen ist hier an der Abszisse der investierte Geldbetrag in die Ausbildung abgetragen und nicht das Bildungsniveau. Dies macht solange keinen Unterschied in der Kurveninterpretation aus, wie jedem Bildungsniveau ein bestimmter Geldbetrag zugeordnet werden kann. Auf der Abzisse kann die Bildungsinvestition dann sowohl in Geldeinheiten als auch in Bildungsstufen abgetragen werden. Zur Vereinfachung der Zusammenhänge wird in den folgenden Ausführungen anhand der Geldeinheiten argumentiert. 
Die obige Finanzierungskurve drückt die marginalen Kosten der Finanzierung einer zusätzlichen Kapitaleinheit aus. Vereinfachend könnte man sagen, dass sie die Zinsrate angibt, die für eine zusätzliche Geldeinheit gezahlt werden muss. 337

Wie bereits in Kapitel 3.1 angeführt wurde, ist die Segmentierung des Kapitalmarktes für die Steigung der individuellen Finanzierungskostenkurve verantwortlich. Jeder Investor wird zuerst die günstigste Quelle zur Finanzierung seiner Ausbildung heranziehen. Erst wenn diese Quelle erschöpft ist, wird er sich der nächsten, teureren Quelle zuwenden. Dies soll beispielhaft für einen Haushalt anhand der obigen Grafik erläutert werden.

Die günstigsten Mittel zur Finanzierung der Ausbildung sind in der Regel Zuwendungen von Verwandten außerhalb des Hauhalts, Stiftungen oder der Regierung, die nur für die Ausbildung der Kinder bestimmt sind. Diese Mittel ziehen keine Kosten für den Haushalt nach sich, da sie entweder für die Ausbildung der Kinder verwendet werden können oder dem Haushalt gar nicht zur Verfügung stehen. Sie sind in der Grafik durch die Strecke Oa gekennzeichnet, weil sie keine Zinskosten verursachen und keiner alternativen Verwendung innerhalb des Haushalts entzogen wurden. ${ }^{338}$ Subventionierte Ausbildungskredite sind etwas teurer und sind in der Grafik durch die Strecke a'b gekennzeichnet. Dann werden freie Ressourcen der Haushalte selbst zur Finanzierung herangezogen ( $b$ 'c). Hierbei kann es sich ebenfalls um Geschenke von Verwandten oder Erbschaften handeln, die an keinen speziellen Zweck gebunden sind. Die Kosten dieser Mittel werden durch ihre Opportunitätskosten (also den entgangenen Nutzen einer alternativen Verwendung) bestimmt. Wegen der hohen Transaktionskosten am Kapitalmarkt sind diese oft günstiger als Kredite zu Marktkonditionen. Erst danach werden die teureren Kredite am Kapitalmarkt nachgefragt bzw. auf Konsum während der Investitionsphase verzichtet. Diese Mittel sind normalerweise zu höheren und schneller steigenden Kosten erhältlich. Sie werden in der Grafik durch den ansteigenden Ast c'S repräsentiert.

Um die Finanzierungssituationen von armen und reicheren Haushalten auch ohne den Einfluss von bildungspolitischen Maßnahmen zu vergleichen, sollen gezielte Stipendien und subventionierte Kredite vernachlässigt werden. Eine Bildungsfinanzierung zum „Nulltarif“ ist damit nur durch Zuwendungen von wohlhabender Verwandtschaft zu erreichen - wenn z.B. reiche Großeltern die Schulbildung der Enkel finanzieren, die Ressourcen sonst aber nicht dem Haushalt zur Verfügung stünden. Für arme Haushalte ist die Wahrscheinlichkeit

337 Vgl. Becker (1983), S. 102.

338 Vgl. Becker (1983), S. $102 f$. 
nicht sehr hoch, dass Teile der Verwandtschaft wohlhabend genug sind, um die Ausbildungskosten ihrer Kinder zu übernehmen. Oft wurde die Armut schon über mehrere Generationen weitergereicht (Teufelskreis der Armut). Außerdem hat die zunehmende Verstädterung in den meisten Entwicklungsländern zu eine Trennung von verwandtschaftlichen Beziehungen geführt. Zweckgebundene Rücklagen für Bildungsausgaben sind in armen Familien kaum zu erwarten. Die Finanzierungskurve für arme Haushalte muss daher in Richtung des Ursprungs verschoben werden, und weist daher schon bei sehr kleinen Beträgen positive Zinskosten auf bzw. beginnt sogar bei einem positiven Zinssatz an der Ordinate.

Subventionierte Kredite dürften bei der Finanzierung der Basisausbildung keine bedeutende Rolle spielen. Die nächst günstigeren Mittel müssten damit aus freien Ressourcen der Haushalte selber gezogen werden. Schon die Definition von Armut339 schließt aus, dass arme Haushalte in großen Mengen über freie Mittel verfügen, die ohne große Opportunitätskosten in die Ausbildung der Kinder gesteckt werden können. Auch Konsumverzicht ist nur unter hohen Opportunitätskosten möglich, da kaum überflüssiger Konsum abgebaut werden kann.

Arme Haushalte haben selten Zugang zu formellen Krediten, da sie keine banküblichen Sicherheiten anbieten können. ${ }^{340}$ Das zu erwerbende Humankapital wird nicht als Gegenwert akzeptiert, da es an die investierende Person gebunden und somit nicht veräußerbar ist. ${ }^{341}$ Ein Bildungskredit ist daher auf dem formellen Kapitalmarkt kaum zu bekommen. Auch der informelle Sektor bietet hier kaum Alternativen, da er keine langfristigen Kredite anbietet. ${ }^{342}$

Aus diesen Gründen muss in armen Familien während des Investitionszeitraumes oft auf Konsum verzichtet werden. Treten in dieser Zeit z.B. durch Krankheiten oder Einkommensschwankungen Bedarfsspitzen auf, müssen diese durch kleine, kurzfristige Kredite bei informellen Geldverleihern ausgeglichen werden. ${ }^{343}$ Die Zinsen im informellen Sektor können zwischen 100\% und über $2500 \%$ betragen. 344

Die Finanzierungskostenkurven zweier Haushalte können sich also sowohl in ihrer Lage als auch in ihrer Steigung unterscheiden. Die Kurven für arme Haushalte beginnen tendenziell bei einem höheren Zinssatz und steigen schneller als

339 Unabhängig von der Art des gewählten Armutsmaßes, sind freie Ressourcen nicht mit dem Begriff der Armut vereinbar.

340 Vgl. WEB 1989, S. 141.

341 Schon Becker weist 1975 auf diese Segmentierung am Kapitalmarkt für Bildungsinvestitionen hin.

342 Wenn überhaupt, werden langfristige informelle Kredite nur zum Bau von Wohnraum angeboten, da ihnen damit eine gewisse Sicherheit gegenübersteht. Vgl. WEB 1989, S. 141.

343 Vgl. WEB 1989, S. 138.

344 Vgl. dazu Hesselbarth/Sautter (1998), S. 356. 
die entsprechenden Kurven wohlhabenderer Haushalte. Während für den einzelnen Haushalt eine Stufenfunktion anzunehmen ist, kann für eine Bevölkerungsgruppe ein stetiger Verlauf der Kurve angenommen werden.

Die folgende Grafik zeigt zwei beispielhafte Verläufe für die beiden Einkommensgruppen.

\section{Grafik 14.2: Finanzierungskostenkurve für arme und reiche Haushalte}

marginale Finanzierungskosten

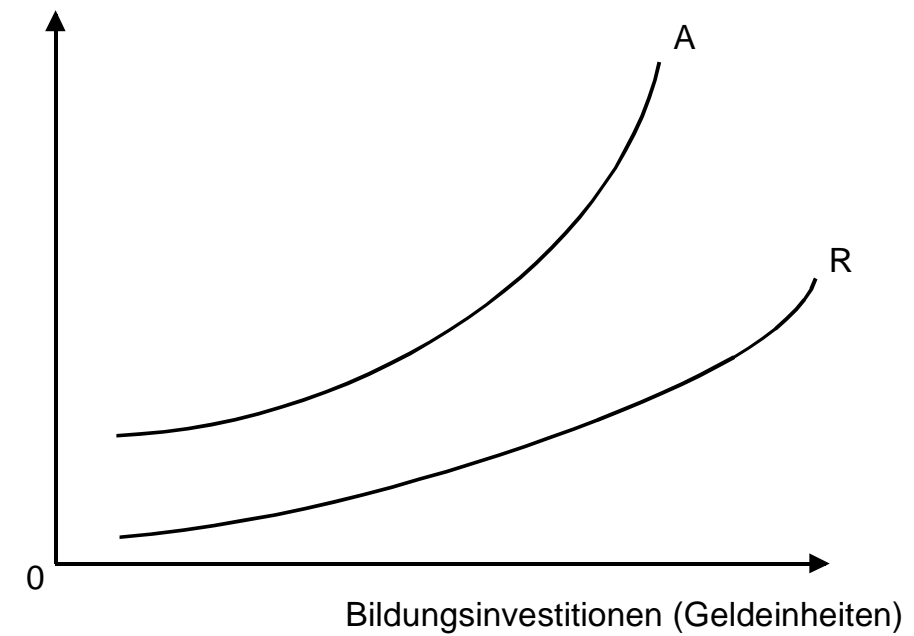

Arme Haushalte müssen also für jede Geldeinheit höhere Zinsen bezahlen als Haushalte aus reichen Familien. Wenn beide Bevölkerungsgruppen für die Ausbildung ihrer Kinder die gleichen Investitionsbeträge aufbringen müssen, heißt das gleichzeitig, dass arme Familien für jedes Bildungsniveau höheren Finanzierungskosten ausgesetzt sind.

Werden jetzt gleiche Ertragsratenfunktionen für beide Bevölkerungsgruppen angenommen, liegt der optimale Investitionspunkt der Armen bei einem niedrigeren Bildungsniveau als der der Reichen. Da aber nicht von identischen Ertragsratenfunktionen ausgegangen werden kann, werden im folgenden die Ertragsratenverläufe genauer untersucht.

\subsubsection{Typische Ertragsratenverläufe}

Die Auswertung der Haushaltsbefragung hat ergeben, dass die Familien der obersten Schicht größere Beträge in die jeweiligen Ausbildungsstufen investieren und auch höhere Einkommenserwartungen an die Ausbildung haben. Sowohl die Kosten als auch die Einkommenserwartungen für die eigenen Kinder der reicheren Haushalte beziehen sich auf die Ausbildung in privaten Institutionen, da fast alle Kinder dieser Schicht auf private Schulen geschickt wurden. Die Angaben der untersten Schicht beziehen sich vor allem auf öffentliche Schulen. 
Wird nun angenommen, dass beide Schichten ihre Kinder auf die gleichen Schulen schicken, fallen Unterschiede in den direkten Kosten der Ausbildung weg. Erstens werden alle Schulen die gleichen Gebühren erheben und die gleichen Ausgaben für Bücher, Uniformen und ähnliches fordern. Zweitens wäre es unsinnig, eine andere Schule zu wählen als die, die dem Wohnort am nächsten liegt, wenn alle Schulen von vergleichbarer Qualität sind. Ist das Angebot an Bildungseinrichtungen so umfassend wie in Lima, werden alle Haushalte in etwa den gleichen Weg zur nächsten Schule haben. Unterschiede in den Transportkosten sind daher ebenfalls nicht zu erwarten.

Bei gleichen Kostenbelastungen lassen sich allein aus den Unterschieden in den Einkommenserwartungen Unterschiede in den Ertragsratenfunktionen konstruieren. Ob die Haushalte tatsächlich unterschiedliche Erwartungen an die zukünftigen Einkommen haben, wenn sich diese auf nur ein Bildungssystem beziehen, kann aus der Haushaltsbefragung entnommen werden.

Neben den zukünftigen Einkommen der eigenen Kinder sollten die Eltern auch die Einkommen von Arbeitnehmern mit unterschiedlichen Bildungsabschlüssen am Arbeitsmarkt schätzen. Ein Vergleich der Daten ergab, dass die reicheren Haushalte das Einkommen der Kinder durchschnittlich etwas höher einschätzten als die Verdienstaussichten am Arbeitsmarkt allgemein. Die Haushalte der untersten Schicht erwarteten für ihre Kinder Einkommen, die leicht unter denen am Arbeitsmarkt allgemein genannt wurden.

Die Diskrepanz zwischen den allgemeinen Einkommen und denen für die eigenen Kinder deutet darauf hin, dass die Eltern der obersten Schicht sich der Vorteile, die sie ihren Kindern (z.B. durch die Privatschule) bieten können durchaus bewusst sind. Obwohl diese Einflüsse auch aus den allgemeinen Angaben für den Arbeitsmarkt nicht ganz auszuschließen sind, kann davon ausgegangen werden, dass es sich dabei um Erwartungen handelt, die sich auf einen Durchschnittsabsolventen des jeweiligen Bildungsniveaus beziehen. Einkommensunterschiede aufgrund von Unterschieden in den besuchten Bildungseinrichtungen sollten hier keine große Rolle spielen.

Während für Personen ohne Ausbildung einheitlich Einkommen um die 300 Soles angegeben wurden, schätzten die Haushalte der obersten Schicht die Einkommen für eine Person mit höherer Bildung auf ca. 3000 Soles im Monat, was einer Steigerungsrate von $870 \%$ entspricht. Die Haushalte der untersten Schicht erwarteten, dass ein Absolvent der höheren Bildung ein Einkommen von ca. 1000 Soles verdient, was einer Steigerung von 258\% entspricht. ${ }^{345}$ Die Differenzen sind also so groß, dass selbst bei einem Resteinfluss der Erfahrun-

345 Vgl. dazu die Ausführungen zu den Ertragserwartungen am Arbeitsmarkt im Kapitel 11.2. 
gen mit dem besuchten Schultyp noch davon ausgegangen werden kann, dass die Ertragserwartungen auch unabhängig vom besuchten Schultyp bei den reicheren Familien optimistischer ausfallen.

Werden diese Erkenntnisse in Ertragsraten für die höhere Bildung ausgedrückt, ergibt sich das folgende Bild.

\section{Grafik 14.3: Typische Ertragsratenkurven für arme und reiche Haushalte}

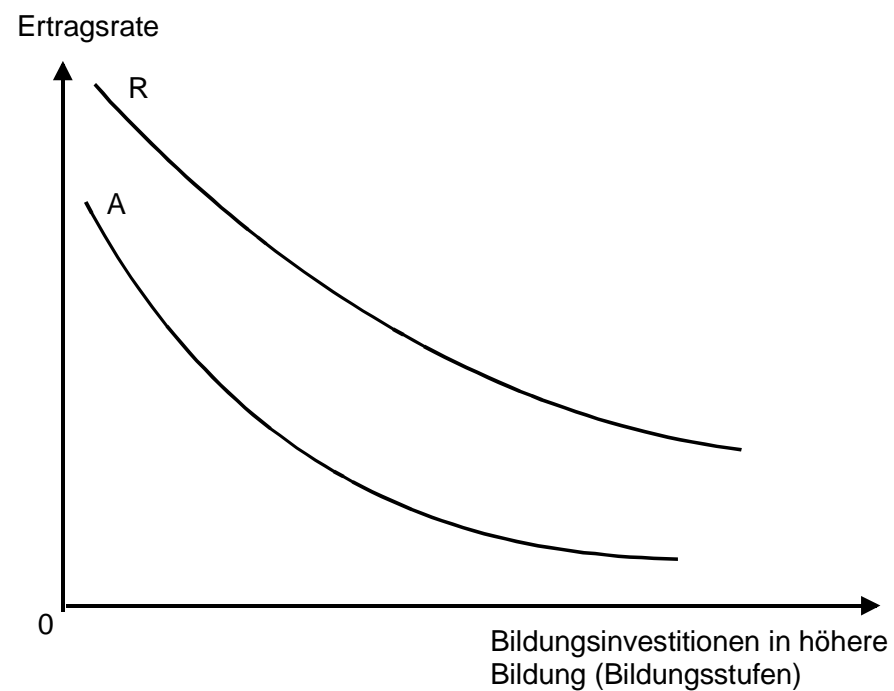

Bei gleichen direkten Kosten der Ausbildung erwarten arme Haushalte aufgrund ihrer niedrigeren Ertragserwartungen bei jedem Bildungsniveau niedrigere Ertragsraten als reiche Haushalte. Im folgenden Abschnitt werden nun die beiden Kurvenverläufe zusammengefügt.

\subsubsection{Optimale Bildungsniveaus für arme und reiche Haushalte}

Unter der Annahme vergleichbarer Schulen kann für arme und reiche Bevölkerungsgruppen je eine typische Ertragsraten- und Finanzierungskurve angenommen werden. Wie die obigen Ausführungen gezeigt haben, liegt die Finanzierungskostenkurve der armen Haushalte über der der reichen Haushalte. Umgekehrt verhält es sich mit den Ertragsratenfunktionen. Obwohl die Finanzierungskostenkurven im Zins-Geldeinheiten-Diagramm konstruiert wurden, können beide Kurven ohne weiteres in einem Diagramm zusammengefügt werden, da mit jedem Schuljahr für beide Einkommensgruppen die gleichen direkten Kosten entstehen. ${ }^{346}$ Im Vergleich zu den Armen weisen reiche Haushalte also bessere Finanzierungsbedingungen gepaart mit höheren Ertragsraten auf. Wie in Kapitel 3 gezeigt wurde, werden derartige Kurvenkombinationen als positiv korreliert bezeichnet. 
Die folgende Grafik zeigt, welche Konsequenzen dies für das optimale Bildungsniveau der beiden Schichten hat.

\section{Grafik 14.4: Optimale Bildungsniveaus für arme und reiche Haushalte}

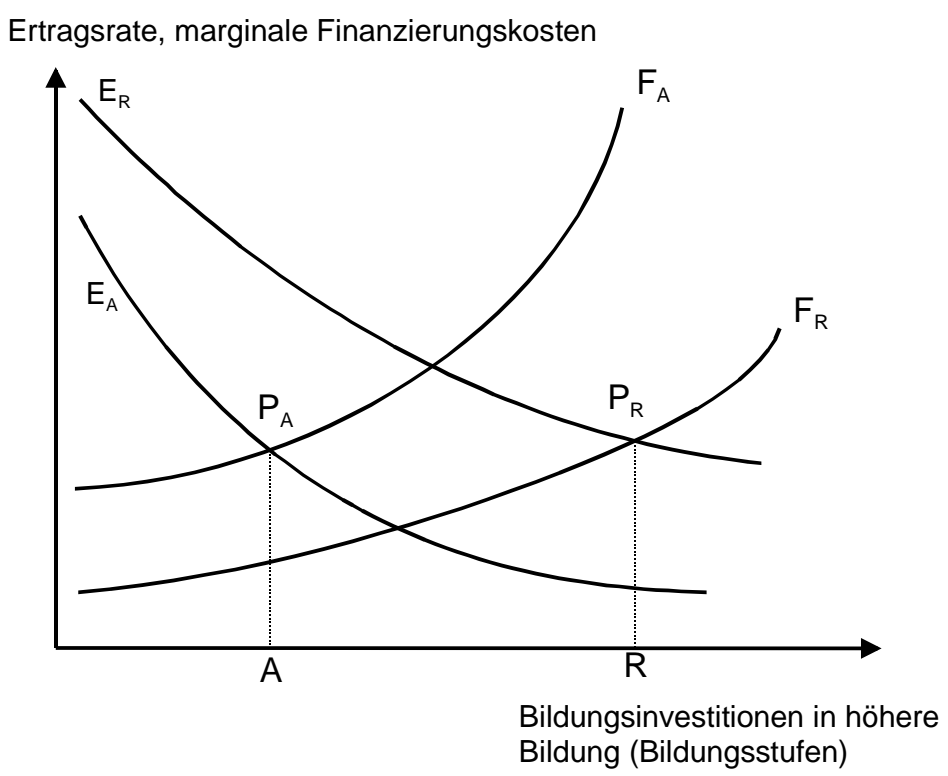

Die beiden Kurven der armen Haushalte treffen sich in Punkt $P_{A}$, was einem Bildungsniveau von $A$ entspricht. Der optimale Investitionspunkt für die reichen Haushalte liegt bei Punkt $R$. Die Grafik zeigt, dass unter den oben genannten Annahmen das durchschnittliche optimale Bildungsniveau von armen Haushalten unter dem reicherer Haushalte liegt. Wenn den Familien rationales Verhalten unterstellt wird, werden arme Familien ein niedrigeres Niveau der höheren Bildung für ihre Kinder nachfragen als wohlhabendere Familien.

Wie in Kapital 3 gezeigt wurde, führt eine positive Korrelation zwischen Ertragsraten- und Finanzierungsbedingungen zu variierenden Bildungsnachfragemengen bei relativ ausgeglichenen Ertragsraten zwischen den Bildungsniveaus. Unter Gültigkeit dieser Annahmen müssten daher unterschiedlich hohe Bildungsniveaus zwischen den Bevölkerungsgruppen beobachtet werden können. Hier sei noch einmal daran erinnert, dass es sich ausschließlich um Investitionsunterschiede im Bereich der höheren Bildung handelt. Die nahezu umfassende und damit ausgeglichene Beteiligung aller Schichten in der Basisausbildung widerspricht diesem Ergebnis also keineswegs.

Ob diese Aussagen Gültigkeit behalten, wenn berücksichtigt wird, dass die reichen Haushalte private Bildungseinrichtungen besuchen wird im folgenden untersucht. 


\subsection{Unter Einbeziehung von privaten Schulen}

Ausbildung wird allgemein als ein Weg zur Verbesserung des Lebensstandards anerkannt. So wirken sich die Faktoren, die die Bildungsentscheidung beeinflussen, auch auf die private und soziale Wohlfahrt eines Landes aus. In fast allen Entwicklungsländern war die Regierung lange Zeit der größte Anbieter von Schulbildung. Weil die Ausbildung sich positiv auf die soziale Wohlfahrt eines Landes auswirkt, wurde die Bereitstellung als typisches öffentliche Aufgabe betrachtet. Die Ausbildung wird mittlerweile aber auch von der Bevölkerung als Investition in die Zukunft betrachtet. Daher haben auch private Anbieter den Investitionswert von Ausbildungseinrichtungen erkannt, und Privatschulen eroberten den Ausbildungsmarkt in fast allen Entwicklungsländern. Die Kosten für den Besuch einer privaten Bildungseinrichtung sind allerdings hoch. Deshalb können sich nur relativ wohlhabende Familien eine private Ausbildung für ihre Kinder leisten. Wie die Haushaltsbefragung zeigte, schicken mittlerweile fast alle Familien der obersten Schicht ihre Kinder in Privatschulen. Der Anteil aus den Familien der untersten Schicht ist dagegen nur gering. ${ }^{347}$

Im folgenden wird davon ausgegangen, dass die reichen Haushalte die Wahl zwischen den beiden Schultypen haben, während die armen Familien ihre Kinder weiterhin auf öffentliche Schulen schicken.

Ausgehend von den obigen Kurvenverläufen bei gleichen Bildungseinrichtungen soll nur gezeigt werden, wie sich diese Kurven für die reichen Haushalte verschieben, wenn sie ihre Kinder auf private Schulen schicken.

\subsubsection{Auswirkungen auf die Ertragsratenkurven}

Wenn sich reiche Haushalte entscheiden, ihre Kinder auf Privatschulen zu schicken, obwohl die öffentlichen Schulen vom Staat zu sehr niedrigen Preisen angeboten werden, ist zu erwarten, dass sie dadurch so hohe zukünftige Einkommenssteigerungen erwarten, dass die Kostendifferenz zwischen den Schulformen mindestens kompensiert wird. Das heißt, dass sie von der privaten Ausbildung höhere Ertragsraten erwarten als sie auf öffentlichen Schulen erreichen könnten. Im Vergleich zu ihrer Ertragsratenfunktion in öffentlichen Bildungseinrichtungen liegt die Funktion der privaten Schulen in jedem Punkt darüber.

347 Vgl. dazu die Befragungsergebnisse zur Bildungssituation der Kinder in Kapitel 10.2. 
Die Grafik zeigt die entsprechenden Kurvenverläufe.

\section{Grafik 14.5: Ertragsraten bei alternativen Schultypen}

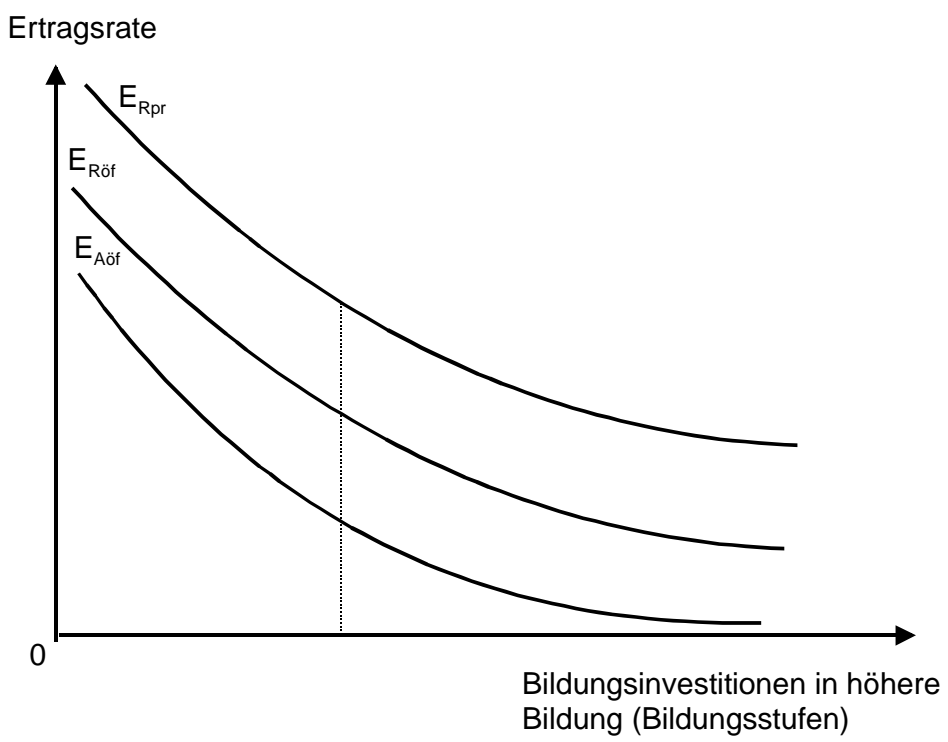

Bei jedem Bildungsniveau liegen die Ertragsraten der reichen Haushalte an privaten Schulen ( $\left.E_{R p r i}\right)$ über denen, die sie für die öffentlichen Schulen erwarten $\left(E_{\text {Röff }}\right)$. Da die Ertragserwartungen der armen Haushalte $\left(E_{\text {Aöff }}\right)$ noch unter denen der Reichen an öffentlichen Schulen liegen, wird der Unterschied in der Lage der Ertragsratenkurven durch den Besuch der Privatschulen noch vergrößert.

Wie weit die Funktion der Privatschulen die der öffentlichen Schulen übersteigt, kann nicht ausgesagt werden. Allerdings müssen die Ertragsraten höher sein, sonst würde sich die Investition in die private Bildung nicht lohnen.

Würde sich für die reichen Haushalte durch den Besuch der Privatschule die Kostensituation nicht ändern, könnten die oben ermittelten Finanzierungskostenkurven einfach in die Grafik integriert werden. Es würde sich zeigen, dass die optimalen Bildungsniveaus der armen und reichen Haushalte noch weiter auseinander liegen, wenn die reichen Haushalte private Schulen besuchen.

Allerdings ändern sich die Kostenbedingungen der reichen Haushalte durch den Besuch der teureren Privatschulen, daher ändern sich auch ihre Finanzierungskostenkurven. Wie sich die Finanzierungsbedingungen der reichen Haushalte genau durch den Besuch der Privatschulen ändern, zeigt der nächste Abschnitt. 


\subsubsection{Auswirkungen auf die Finanzierungskostenkurven}

Durch den Besuch einer Privatschule kommen auf die Familien deutlich höhere Kosten zu. Erstens sind die Immatrikulationsgebühren hoch. Zweitens sind die privaten Bildungseinrichtungen noch nicht so verbreitet, dass in jedem Wohngebiet eine private Schule erwartet werden kann. Die Transportkosten können daher ebenfalls als höher angenommen werden. In diesem Fall sind die investierten Beträge nicht eindeutig einem Bildungsniveau zuzuordnen

Wie aus den obigen Ausführungen hervorging, haben reiche Familien niedrigere Finanzierungskosten als arme Familien bei gleichen Geldbeträgen. Zur Vereinfachung sollen auch hier wieder die Kosten der Finanzierung einer zusätzlichen Kapitaleinheit als Zinsrate bezeichnet werden. Wenn reiche Eltern ihre Kinder an private Schulen schicken, müssen sie für jedes Bildungsniveau größere Summen investieren als in öffentlichen Schulen. Steigen die Finanzierungskosten mit jeder Kapitaleinheit, heißt dass, dass die reichen Familien bei jedem Bildungsniveau an Privatschulen höhere Zinsen zu zahlen haben als bei dem gleichen Bildungsniveau an öffentlichen Schulen. Ihre Finanzierungskostenkurve steigt damit an und nähert sich der für die armen Familien. Es ist sogar möglich, dass sie diese erreicht oder sogar übersteigt.

Mit der Integration der Kurve für die privaten Bildungseinrichtungen kann die Achsenbezeichnung der Abzisse nicht mehr einfach variiert werden, da sie sich bei gleichen Bildungsniveaus auf unterschiedliche Finanzierungskosten bezieht. Die Grafik zeigt, wie anhand eines Beispiels der Verlauf der neuen Kurve abgeleitet werden kann.

\section{Grafik 14.6: Finanzierungskosten bei alternativen Schultypen}

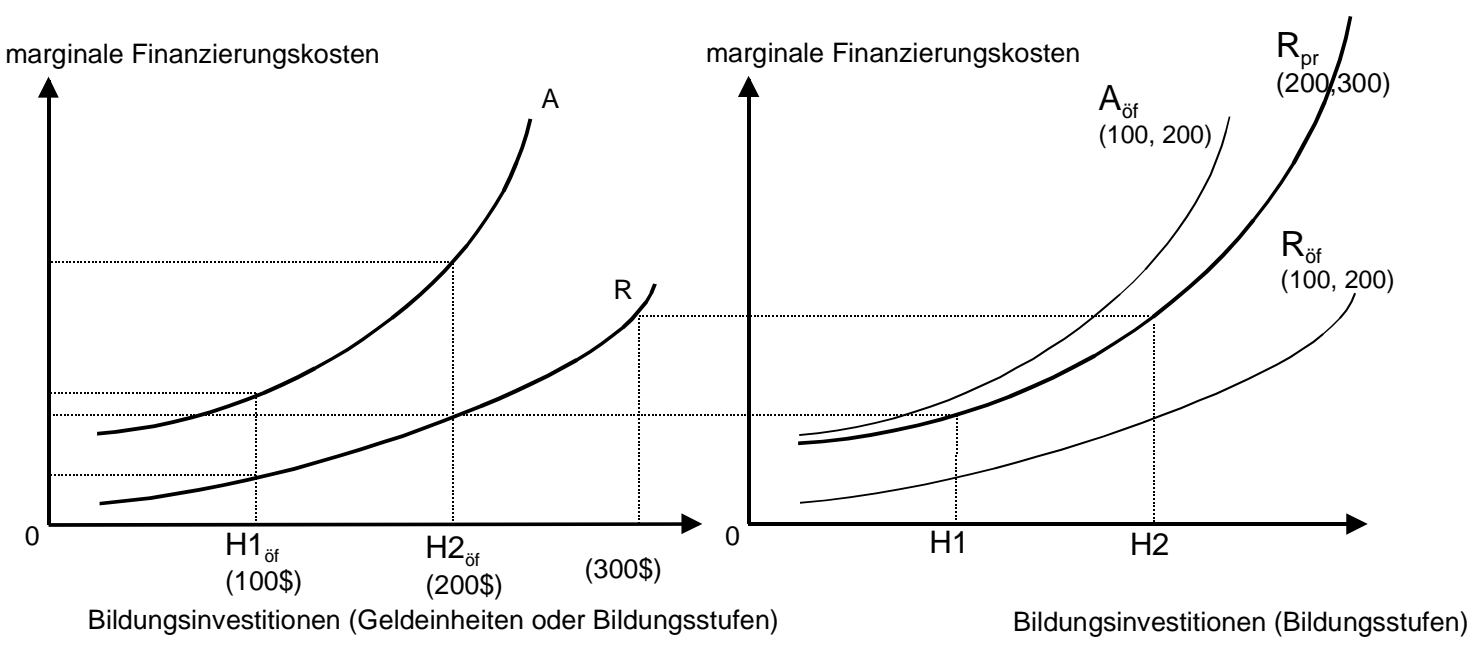

Wenn alle Kinder auf die gleichen Schulen gehen, müssen alle Familien die gleichen Kosten für ein bestimmtes Bildungsniveau aufbringen. Zu einem Bil- 
dungsniveau gehört dann ein eindeutiger Preis. An der Abzisse können die Bildungsinvestitionen sowohl in Bildungsstufen als auch in Geldeinheiten abgetragen werden. Die von oben bekannte Darstellung befindet sich auf der linken Seite der Grafik. Anhand der Finanzierungskostenkurven kann abgelesen werden, wie hoch die Finanzierungskosten für beide Hauhalte bei alternativen Investitionsbeträgen sind.

In der rechten Grafik kann nun eine beispielhafte Finanzierungskostenkurve der reichen Familien für die Privatschule konstruiert werden. Angenommen, in einer privaten Bildungseinrichtung kostet die Bildungsstufe $H 1200 \$$ und die Bildungsstufe $\mathrm{H} 2300$. Dann kann anhand der Information aus der linken Grafik ermittelt werden, wo die dazu gehörige neue Kurve liegen muss. Zum Vergleich sind die beiden Kurven für öffentliche Schulen dazukopiert. Da den Bildungsstufen nun kein eindeutiger Investitionsbetrag mehr zugeordnet werden kann, kann an der Abzisse nunmehr ausschließlich die Bildungsstufe abgetragen werden. Den Kurven liegen unterschiedlich hohe Investitionsbeträge zugrunde.

In diesem Beispiel liegt die Kurve für private Schulen der reichen Haushalte über ihrer Kurve für öffentliche Schulen aber immer noch unter der Kurve der armen Haushalte für öffentliche Schulen. Je nach Kostendifferenz zwischen öffentlichen und privaten Schulen kann die Kurve für Privatschulen aber auch stärker oder weniger stark steigen.

Wie sich diese Veränderungen der Kurven auf die optimalen Bildungsniveaus auswirken können, wird im folgenden Abschnitt behandelt.

\subsubsection{Auswirkungen auf die optimalen Bildungsniveaus}

Die obigen Ausführungen haben ergeben, dass die Ertragsratenfunktion der Reichen für private Schulen ihre Ertragsratenfunktion für öffentliche Schulen noch übersteigt und damit die Diskrepanz zu der Ertragsratenkurve der Armen noch verstärkt wird. Bei den Finanzierungskostenkurven ist eher eine Annäherung der Kurven der beiden Bevölkerungsgruppen zu erwarten, da der Zinsvorteil der reichen Haushalte durch die höheren Kosten der privaten Bildung reduziert wird. Da die genaue Lage der Finanzierungskostenkurve für die Privatschule zu der Finanzierungskostenkurve der Armen nicht eindeutig bestimmt werden konnte, sind in folgender Grafik einige mögliche Verläufe eingezeichnet. Die Kurven $F_{R 1}$ bis $F_{R 4}$ geben alternative Verläufe für die Finanzierungskostenkurve des reichen Haushalts für die Privatschule an. Je stärker seine Kapitalkosten mit jeder zusätzlichen Geldeinheit steigen, desto höher verläuft die Fi- 
nanzierungskostenkurve für die teurere Ausbildung. ${ }^{348}$ Hat der Haushalt eine niedrige und flache Kurve im Zins-Geldeinheiten-Diagramm vorzuweisen, könnte sich für ihn die Kurve $F_{R 1}$ für die private Ausbildung ergeben. Steigt der Finanzierungszins für den Haushalt allerdings sehr stark mit jeder zusätzlichen Geldeinheit an, könnte er sogar Finanzierungskosten für die private Ausbildung in der Form der Kurve $F_{R 4}$ aufweisen.

\section{Grafik 14.7: Mögliche Verläufe der Finanzierungskostenkurve}

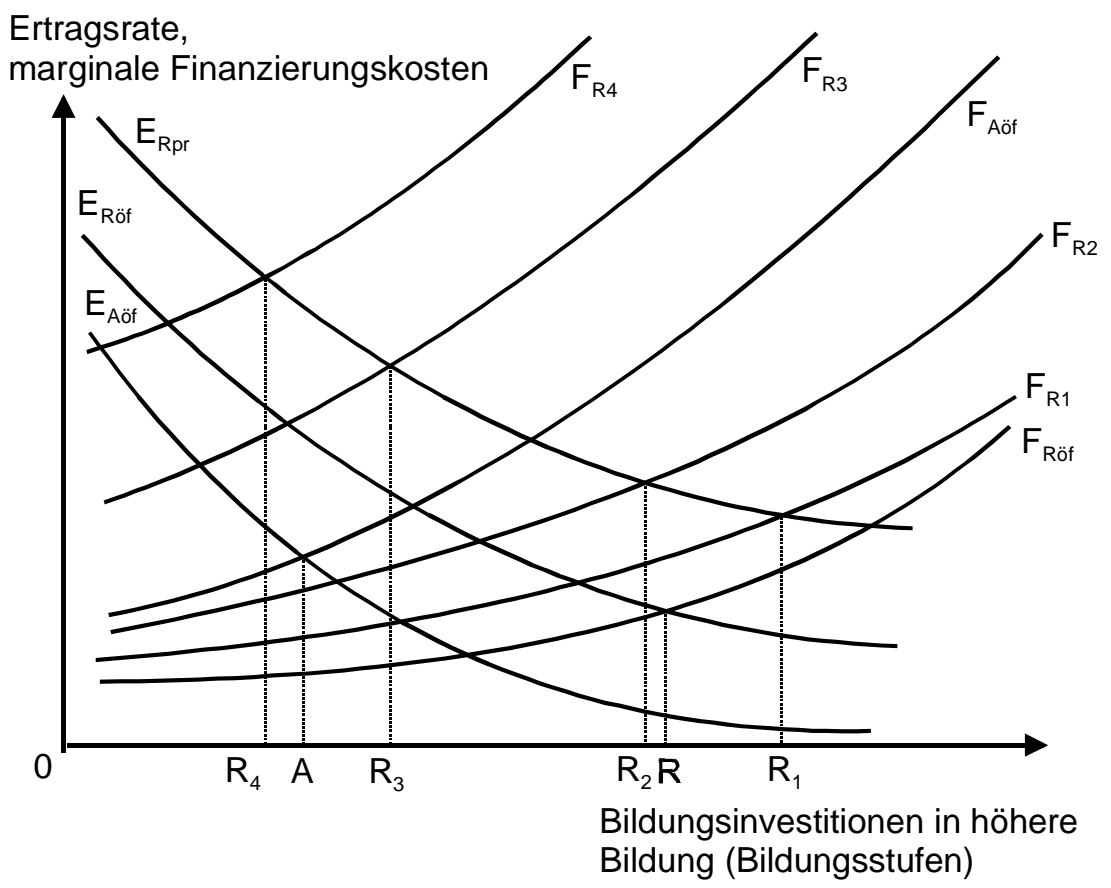

Wenn beide Bevölkerungsgruppen ihre Kinder auf öffentliche Schulen schicken, ergeben sich die optimalen Bildungsinvestitionen an den Punkten $A$ (arm) und $R$ (reich). Die Grafik zeigt nun, dass sich das Optimum für den reichen Haushalte noch weiter nach außen verschiebt, wenn die Ertragsraten durch den Besuch der privaten Schule stärker steigen als die Finanzierungskosten. Es ergibt sich ein optimales Bildungsniveau in Punkt $R_{1}$. Der Haushalt würde seine Bildungsnachfrage durch den Besuch einer privaten Schule sogar noch ausweiten und damit die Bildungsdiskrepanz zu den armen Haushalten vergrößern. Steigen allerdings die Finanzierungskosten stärker als die Ertragsraten durch den Besuch einer privaten Schule, verschiebt sich die Finanzierungskostenkurve stärker nach oben. Das optimale Bildungsniveau verschiebt sich nach links auf $R_{2}$, und der Haushalt wird auf einer privaten Schule weniger Bildung nachfragen als auf der öffentlichen. Die Diskrepanz in den Nachfragemengen zwischen Arm und reich würde damit geschmälert.

348 Vergleich hierzu noch mal die typischen Verläufe der Finanzierungskostenkurven für arme und reiche Haushalte in Grafik 13.2 und die Ableitung der Kurve aus dem Zins-Geldeinheiten-Diagramm in das Zins-Bildungsstufen-Diagramm in Grafik 14.6. 
In beiden Fällen liegt die Finanzierungskostenkurve des reichen Haushaltes aber unter der des armen Haushaltes. Auch beim Besuch unterschiedlicher Schultypen sind die Finanzierungs- und die Ertragsratenbedingungen also noch positiv korreliert. Das heißt, bessere Finanzierungsbedingungen treffen mit höheren Ertragsraten zusammen.

Steigen nun aber die Finanzierungskosten durch den Besuch der privaten Schule so stark an, dass die neue Kurve für den reichen Haushalt über der Kurve des armen Haushalts liegt, sind die Finanz- und Ertragsbedingungen negativ korreliert. Das heißt, der reiche Haushalt hat zwar eine höher verlaufende Ertragsratenfunktion aber dafür schlechtere Finanzbedingungen für ein gegebenes Bildungsniveau aufzuweisen als der arme Haushalt.

Bei der Finanzierungskostenkurve $F_{R 3}$ überwiegen allerdings die besseren Ertragsratenbedingungen des reichen Haushalts seine schlechteren Finanzierungsbedingungen. Sein Bildungsoptimum liegt zwar niedriger als beim Besuch einer öffentlichen Schule aber immer noch über dem des armen Haushalts. Erst wenn die hohen Zinsen durch den großen Investitionsaufwand die Vorteile der höheren Ertragsraten übersteigen, senkt sich das optimale Investitionsniveau des reichen Haushaltes unter das des armen Haushalts. Sein Bildungsoptimum liegt dann im Punkt $R_{4}$ und er wird sogar weniger Bildung nachfragen als der arme Haushalt an der öffentlichen Schule.

Theoretisch sind also alle angegebenen Konstellationen möglich. Allerdings wurde oben bereits ermittelt, dass die Zinsfunktionen der reichen Haushalte relativ niedrig und flach verlaufen. ${ }^{349}$ Ein derart extremer Verlauf der Finanzierungskostenkurve wie in $F_{R 4}$ ist daher nicht wahrscheinlich. Die Lage der Kurve wird allerdings auch durch die Kosten der privaten Ausbildung bestimmt. Selbst bei nur moderat ansteigenden Zinsen mit jeder zusätzlichen Geldeinheit, kann es zu weit nach oben verschobenen Finanzierungskostenkurven kommen, wenn die private Bildung zu Kosten führt, die weit genug über denen der öffentlichen Ausbildung liegen.

Allerdings ist die private Ausbildung eine freiwillige Alternative zu der öffentlichen Ausbildung. Es kann nicht davon ausgegangen werden, dass sich die Eltern für eine Bildungsalternative entscheiden, deren Kosten so hoch sind, dass sich das optimale Bildungsniveau für ihre Kinder derart nach unten verschiebt wie z.B. in Punkt $R_{4}$. Die Familien werden sich kaum entscheiden, eine private Hochschule zu besuchen, wenn das Kind diese wieder verlassen müsste, bevor es den auf einer öffentlichen Universität angestrebten Abschluss erreicht hätte.

349 Vgl. 14.1.1 zu den typischen Kurvenverläufen von armen und reichen Haushalten. 
Realistischerweise kann erwartet werden, dass auch beim Besuch verschiedener Bildungseinrichtungen das optimale Bildungsniveau und damit die Nachfrage armer Haushalte unter dem der reicheren Haushalte zurückbleibt.

Hier sei noch einmal darauf hingewiesen, dass sich die oben dargestellten Ergebnisse nur auf die Investitionsentscheidung bezüglich einer höheren Ausbildung beziehen. Die Basisausbildung unterliegt einem derartigen Kalkül nicht. Sie wird als notwendige Voraussetzung für weitere Bildung betrachtet. Die Beteiligung an dieser Ausbildungsstufe ist über alle Schichten hinweg als nahezu umfassend zu bezeichnen. Das Problem in diesem Bereich ist nicht die zu geringe Beteiligung armer Haushalte, sondern die schwachen Leistungen der Schüler und die hohen Wiederholungsraten. 


\subsection{Zusammenfassung der Ergebnisse und Schlussfolgerungen}

Die vorangehende Untersuchung bietet interessante Ergebnisse für die Bildungsnachfrage verschiedener Bevölkerungsgruppen in Lima.

Die Basisausbildung wird vor allem als notwendige Voraussetzung für weitere Bildung betrachtet. Sie hat für sich genommen keinen ökonomischen Wert, da sie zu Verlusten führt. Erst mit der Option, anschließend Zugang zu einer höhere Bildungseinrichtung zu bekommen, wird ihr überhaupt ein Wert zuerkannt. Der erste ökonomisch relevante Abschluss des Bildungssystems scheint ein Abschluss einer höheren Bildung zu sein. Das Optimierungskalkül zur Ermittlung der optimalen Ausbildungsmenge setzt daher erst bei der höheren Bildung an.

Die theoretische Darstellung der Bildungsnachfrage nach höherer Ausbildung zeigt, dass für reiche und arme Bevölkerungsgruppen unterschiedliche durchschnittliche Kurvenverläufe angenommen werden müssen. Bei dem Besuch vergleichbarer Bildungsinstitutionen lässt sich zeigen, dass die Finanzierungsund die Ertragsbedingungen positiv korreliert sind. Die optimalen Bildungsniveaus für reiche und arme Haushalte liegen weit auseinander. Wenn sich alle Bevölkerungsschichten nach einem ökonomischen Kalkül für ihre Bildungsinvestition entscheiden, werden reiche Haushalte deutlich mehr höhere Bildung nachfragen als arme Haushalte.

Wenn sich die Konditionen durch die Wahl extrem teurer privater Bildungseinrichtungen nicht komplett umkehren (was nicht zu erwarten ist), muss auch unter Einbeziehung der privaten Ausbildung der reicheren Haushalte damit gerechnet werden, dass rationales Handeln der Individuen zu Unterschieden in der Nachfrage nach höherer Bildung führt. Das heißt, dass in den Tendenz Kinder reicher Haushalte erstens qualitativ höherwertige Ausbildungseinrichtungen besuchen und zweitens höhere Bildungsabschlüsse erreichen. Der Bildungsrückstand armer Bevölkerungsgruppen wird dadurch perpetuiert.

Die Unterschiede basieren vor allem auf den Erwartungen über die zukünftigen Einkommen und der Finanzierungssituation der Haushalte. Während die Finanzierungssituation der armen Haushalte definitionsgemäß als schlechter angenommen wird, muss die Erwartungsbildung zwischen den verschiedenen Bevölkerungsgruppen noch ausgiebiger erforscht werden. Für Lima ergaben sich immense Unterschiede in den Erwartungen zwischen den Einkommensschichten.

Die individuellen Erwartungen (ob sie realistisch sind oder nicht) sind es, die die private Bildungsnachfrage beeinflussen. Daher sollte ihrer Kenntnissen in der 
Bildungspolitik hohe Bedeutung beigemessen werden. Nur wenn diese Mechanismen bekannt sind, können die richtigen Instrumente identifiziert werden, um die festgesteckten Ziele der Bildungspolitik zu erreichen.

Sollten die Einkommenserwartungen nicht der Realität am Arbeitsmarkt entsprechen, bieten sich der Bildungspolitik Ansatzpunkte, um über eine verbesserte Informationspolitik die Bildungsnachfrage zu beeinflussen.

Sollten sich die Erwartungen allerdings als realistisch herausstellen, sollten die Ziele in der Bildungspolitik u. U. angepasst werden.

Wie schon erwähnt, wird für die Basisausbildung kein ökonomischer Wert erwartet. Sie wird von den Haushalten nur wahrgenommen, weil sie die notwendige Voraussetzung für weitere Bildung oder weil die Teilnahme verpflichtend ist. Für alle Individuen, die wissen, dass sie ihren Bildungsweg nach Abschluss der Sekundaria nicht fortsetzen werden, ist die Basisausbildung eine lästige Pflicht. Diese Einstellung kann negative Auswirkungen auf die Leistungsmotivation haben. Dementsprechend ist das Problem bei der Basisausbildung nicht die mangelnde Teilnahme armer Bevölkerungsgruppen, sondern deren schlechte Leistungen und hohe Wiederholerraten. Von einer Ausweitung des Bildungsangebots sind daher keine Erfolge zu erwarten.

Wenn die negativen Erwartungen der Haushalte der Realität entsprechen, hat die Basisausbildung keinen privatwirtschaftlichen Wert. Ein Grund für die niedrigen Erträge der Basisausbildung kann die niedrige Qualität der Ausbildung sein. Die Diskrepanz zwischen eingesetztem Kapital und Bildungsoutput ist vor allem in Entwicklungsländern sehr groß. ${ }^{350}$ In Peru ist die schlechte Lehrerausbildung und die geringe Motivation der Lehrer durch das Entlohnungssystem ein wertvoller Ansatzpunkt für die Bildungspolitik. ${ }^{351}$

Kann der privatwirtschaftliche Wert der Basisausbildung nicht erhöht werden, kann die Schulpflicht zu hohen privaten Verlusten führen. Sind allerdings die sozialen Erträge der Basisausbildung so hoch, dass eine umfassende und erfolgreiche Beteiligung der Bevölkerung an dieser Bildungsstufe erwünscht ist, sollten zumindest die Haushalte subventioniert werden, denen der Zugang zu höherer Bildung verwehrt bleibt, oder ihnen sollte der Zugang zu höherer Bildung ermöglicht und kommuniziert werden.

Hier ist allerdings darauf hinzuweisen, dass die Öffnung der höheren Bildungseinrichtung für alle Bevölkerungsgruppen noch kein Garant für den Abbau sozialer Ungleichheiten ist. Für Industrieländer wurden dazu Studien mit interessan-

350 Zu Lateinamerika vgl. Schiefelbein (1992), S. 15-26 und Wolff/Schiefelbein/Valenzuela (1994), S. 102. 351 Vgl. Weltbank (2001), S. 47-55. 
ten Ergebnissen durchgeführt. ${ }^{352}$ Die Bildungsexpansion in der zweiten Hälfte des vorigen Jahrhunderts hat zwar zur Öffnung der Hochschule geführt, diese Verbreiterung der Basis hatte aber keinen Effekt auf die Besetzung von Führungspositionen in der deutschen Wirtschaft. ${ }^{353}$ MÜLLER-BENDICT stellte sogar fest, dass die soziale Herkunft gerade dann wieder an Bedeutung zunimmt, wenn ihr Einfluss bei der Bildung über das Erreichen hoher Chancengleichheit im Bildungssystem zurückgedrängt erscheint. ${ }^{354}$ HARTMANN weist in seiner Untersuchung nach, dass bei gleichem Bildungsabschluss (er untersuchte den Zusammenhang anhand der Promotion) die soziale Herkunft dafür entscheidend ist, wer eine Führungsposition bekommt. Seiner Meinung nach ist ab einer bestimmten Hierarchiestufe vor allem der Habitus entscheidend. 355

Anhand dieser Erkenntnisse ist durchaus zu vermuten, dass die Einkommenserwartungen der limenser Haushalte der Realität am Arbeitsmarkt entsprechen. Liegen die Einkommensunterschiede tatsächlich vor allem im Habitus und damit in der sozialen Herkunft begründet, kann eine Öffnung der höheren Bildung keinen großen Effekt auf die soziale Mobilität haben. Sollten die Einkommensunterschiede allerdings vor allem auf Produktivitätsunterschieden beruhen, weil die reicheren Haushalte deutlich bessere Ausbildungsqualitäten genießen, kann eine Erhöhung der Bildungsnachfrage der armen Haushalte erreicht werden, indem die Bildungsqualität in den öffentlichen Schule erhöht wird.

Für die Bildungspolitik ist die Kenntnis der hier behandelten Ertragserwartung also von großer Bedeutung. Zum einen haben sie direkten Einfluss auf die Wirkung von bestehenden bildungspolitischen Maßnahmen, zum anderen sollten sie Einfluss auf die Auswahl dieser Maßnahmen haben. Dabei ist die Kenntnis der Realitätsnähe der Erwartungen unabdingbar. Außerdem kann die Kenntnis der Zusammenhänge dazu beitragen, den möglichen Wirkungsspielraum von Bildungspolitik zu realisieren, und damit zur Formulierung realisierbarer Ziele beitragen.

\footnotetext{
352 Vgl. zum Beispiel Hartmann/Kopp (2001), Hartmann (2002), Müller-Benedict (1999).

353 Vgl. Hartmann/Kopp (2001), S. 436-466.

354 Müller-Bendict (1999), S.328.

355 Hartmann (2002).
} 



\section{Literaturverzeichnis}

Almeida, Heitor/Camargo, José M. (1994): Human Capital Investment and Poverty, Texto para Discussao No.319, Maio 1994.

Amemiya, Takeshi (1981): Qualitative Response Models: A Survey; in: Journal of Economic Literature, Vol. XIX, December 1981, S. 1483-1536.

Arnold, Lutz: Neue Wachstumstheorie: Ein Überblick, in: IFO Studien, Jhg. 41, Nr. 3, 1995, S. 409-445.

Baaden, Andreas (2002): Bildung für Alle bis 2015? Die UNESCO und der Aktionsplan von Dakar, in: DSE (Hrsg):E+Z - Entwicklung und Zusammenarbeit, Nr.8/9, September 2002, S. 246-248.

Backhaus, K./Erichson, B./Plinke, W./Schuchard-Ficher, Chr./Weiber, R.: Multivariate Analysemethoden, 4. Auflage, Berlin u.a. 1984.

Barham, Vicky/Boardway, Robin/Marchand, Maurice/Pestieau, Pierre (1995): Education and the Poverty Trap, in: European Economic Review 39, 1995, S. 1257-1275.

Becker, Gary S. (1975): Human Capital - A Theoretical and Empirical Analysis, with Special Reference to Education, Chicago and London 1975.

Becker, Gary S. (1976): The Economic Approach to Human Behavior, Chicago 1976.

Becker, Gary S. (1983): Human Capital - A Theoretical and Empirical Analysis, with Special Reference to Education, 2. ed., Midway reprint, Chicago and London 1983.

Becker, Gary S./Lewis, Gregg H. (1973): On the Interaction between the Quantity and Quality of Children, in: Becker, Gary S.: The Economic Approach to Human Behavior, Chicago 1976, S. 195-204.

Becker, Gary S./Tomes, Nigel (1976): Child Endowments and the Quantity and Quality of Children, in: Journal of Political Economy, Vol. 84, 1976, No. 4, Part 2, S. 143-162.

Bedi, Arjun S./Marshall, Jeffrey H. (1999): School Attendance and Student Achievement: Evidence from Rural Honduras, in: Economic Development and Cultural Change, Vol. 47, No. 3, April 1999, S. 657-682.

Ben-Porath, Yoram (1967): The Production of Human Capital and the Life Cycle of Earnings, in: Journal od Political Economy, Vol. 75, Part I, No. 4, August 1967, S. 352-365. 
Bennell, Paul (1996):Rates of Return to Education: Does the Conventional Pattern Prevali in Sub-Saharan Africa?; in: World Development, Vol. 24, No. 1, S. 183-199.

Betts, Julian R. (1996): What Do Stdents Know About Wages?, in: The Journal of Human Resources, Vol. 31, No. 1, Winter 1996, S. 27-56.

Biondi Shaw, Luis (1997): Economía de la Educación, Lima 1997.

Birdsall, Nancy (1985): Public Inputs and Child Schooling in Brazil, in Journal of Development Economics, 18 (1985), S. 67-86.

Björklund, Anders/Kjellström, Christian (1999): Estimating the Rate of Return to Investment in Education: How useful is the Standard Mincer Equation?, in: Kjellström: Essays in Investment in Human Capital, Swedish Institute for Social Research, No.36, Edsbruk 1999.

Blau, Francine D./Ferber, Marianne A. (1991): Career Plans and Expectations of Young Women and Men - The Earning Gap and Labour Force Participation, in: The Journal of Human Resources, Vol. 26, No. 4, 1991, S. 581607.

Blaug, Mark (1991): An Introduction to the Economics of Education, 1991.

Blaug, Mark (1992): The Economic Value of Education, Cambridge 1992.

Bowman, Mary J. (1962): Returns to Education, in: Economics of Education, International Social Science Journal (UNESCO), Vol. XIV, 1962, S. 647659.

Burney, Nadeem A./Irfan, Mohammad (1995): Determinants of Child School Enrolment: Evidence from LDCs using choice-theoretic approach, in: International Journal of Social Economics, Vol. 22, No. 1, 1995, S. 24-40.

Busse von Colbe/Laßmann(1990): Betriebswirtschaftstheorie 3 - Investitionstheorie, Berlin, Heidelberg 1990, S. 105-127.

Clotfelter, Charles T./Rothschild, Michael (Hrsg.)(1993): Studies of Supply and Demand in Higher Education, London, Chicago 1993.

Cuánto S.A. (1993): Cómo Estamos?, No. 3, Vol. 1, 1993.

Cuánto S.A. (1997): Perú an Números 1996, Lima 1997. 
Dabla-Norris, Era/Matovu, John M./Wade, Paul (2002): Dept Relief, Demand for Education, and Poverty, WIDER Discussion Paper No. 2002/52, Juni 2002.

Das, Marcel/van Soest, Arthur (2000): Expected versus Realized Income Changes: A Test of the Rational Expectations Hypothesis, Center for Economic Research, No. 2000-105, October 2000.

De Vreyer, Philippe (1993): Une Analyse Ėconométrique de la Demanda d‘ Èducation en Cote-d'Ivoire, in: Revue d'Èconomie du Développement, 3/1993, S. 49-79.

Demery, Lionel/Ferroni, Marco/Grootaert, Christiaan (1993): Understanding the Social Effects of Policy Reform, Washington D.C., 1993, Box 11.6.

Díaz Díaz, Hugo (1994): El Sistema Educativo en América Latina: El Caso del Perú, in: Contribuciones, No. 4, 1994, S. 41-53.

Dominitz, Jeff/Manski, Charles F. (1996): Eliciting Students Expectations of the Returns to Schooling, in: The Journal of Human Resources, Vol. 31, No. 1, Winter 1996, S. 1-26.

DSE (Hrsg.)(2002): E+Z - Entwicklung und Zusammenarbeit, 43. Jg., Nr. 8/9, August/September 2002, Frankfurt/M.

Elster, John (1979): Ulysses and the Sirens: Studies in Rationality and Irrationality, Cambridge u.a. 1979.

Foro Educativo (1997a): La Educación Secundaria de Adolescentes en el Perú - Realidad y Propuesta de Desarrollo Pedagógica, Lima 1997.

Foro Educativo (1997b): Bases para un Acuerdo Nacional por la Educación Peruana, Lima, Octubre 1997.

Foro Educativo (1997c): Desde los Ninos/as - Análisis de las Políticas Educativas 1995-1997, Lima 1997.

Gambetta, Diego (1987): Were They Pushed or Did They Jump?- Individual Decision mechanisms in education, Cambridge u.a. 1987.

Gertler, Paul/Glewwe, Paul (1992): The Willingness to Pay for Education for Doughters in Contrast to Sons: Evidence from Rural Peru, in: The World Bank Economic Rewiev, Vol. 6, No. 1, 1992, S. 171-181. 
Glewwe, Paul (1996): The relevance of standard estimates of rates of return to schooling for education policy: A critical assessment, in: Journal of Development Economics, Vol. 51, S. 267-290.

Glewwe, Paul (2002): Schools and Skills in Developing Countries: Education Policies and Socioeconomic Outcomes, in: Journal of Economic Literature, Vol. XL, June 2002, S. 436-482.

Glewwe, Paul/Grosh, Margaret/Jacoby, Hanan/Lockheed, Marlaine (1995): An Eclectic Approach to Estimating the Determinants of Achievement in Jamaican Primary Education, in: The World Bank Economic Review, Vol. 9, No. 2, 1995, S. 231-258.

González, Xulia/Miles, Daniel (2001): Wage Inequality in a Developing Country: Decrease in Minimum Wage or Increase in Education Returns, in: Empirical Economics, Vol. 26, S. 135-148, 2001.

Gottschalk, Ingrid (2001): Meritorische Güter und Konsumentensouveränität Aktualität einer konfliktreichen Beziehung, in: Jahrbuch für Wirtschaftswissenschaften, Bd. 52, Nr. 2, 2001, S.152-170.

Gould, Davis M./Ruffin, Roy J. (1995): Human Capital, Trade, and Economic Growth, in: Weltwirtschaftliches Archiv, Band 131, Heft 3, 1995, S. 425445.

Handa, Sudhanshu (1996): The Determinats of Teenage Schooling in Jamaica: Rich vs Poor, Females vs Males, in: The Journal of Development Studies, Vol. 32, No. 4, April 1996, S. 554-580.

Hanushek, Eric A. (1987): Educational Production Functions, in: Psacharopoulos, George: Economics of Education - Research and Studies, 1987, S. 33-41.

Hanushek, Eric A. (1993): Comment, in: Clotfelter, Charles T./Rothschild, Michael (Hrsg.): Studies of Supply and Demand in Higher Education, London, Chicago 1993, S. 57-60.

Hanushek, Eric A. (1995): Interpreting Recent Research on Schooling in Developing Countries, in: The World Bank Research Observer, Vol. 10, No. 2, August 1995, S. 227-246.

Harbison, Ralph W./Hanushek, Eric A. (1992): Educational Performance of the Poor: Lessonsfrom Rural Northeast Brazil, Oxford, New York 1992. 
Hartmann, Michael (2002): Der Mythos von den Leistungseliten - Spitzenkarrieren und soziale Herkunft in Wirtschaft, Politik, Justiz und Wissenschaft, Frankfurt/Main 2002.

Hartmann, Michael/Kopp, Johannes (2001): Elitenselektion durch Bildung oder durch Herkunft? Promotion, soziale Herkunft und der Zugang zu Führungspositionen in der deutschen Wirtschaft, in: Kölner Zeitschrift für Soziologie und Sozialpsychologie, Jg. 53, Heft 3, S. 436-466.

Haveman, Robert/Wolfe, Barbara (1995): The Determinants of Children's Attainments: A Review of Methods and Findings, in: Journal of Economic Literature, Vol. XXXIII, December 1995, S. 1829-1878.

Hesselbarth, Susanne/Sautter, Hermann (1998): Strategien zur intensiven Nutzung des Faktors Arbeit als Beitrag zur Armutsminderung, in: GTZ: Armutsorientierte Wirtschaftspolitik, Dokumentation der Fachtagung vom 10.-14.11. 1997, S.327-392, Eschborn 1998

Hicks, N.L. (1987): Education and Economic Growth, in: Psacharopoulos, George (Hrsg.): Economics of Education - Research and Studies, Oxford u.a. 1987, S. 101.107.

Hicks, David (1996): A Lesson for the Future - Young People's Hopes and Fears for Tomorrow, in: Futures, Vol. 28, No. 1, 1996, S. 1-13.

Hirshman, Albert O. (1970): Exit, Voice, and Loyalty: Responses to Decline in Firms, Organizations, and States, Cambridge u.a. 1970.

Hofmann, Michael/ Lock, Stefan (2002): Von Dakar bis PISA - Zukunftsinvestition in Bildung, in: DSE (Hrsg.):E+Z - Entwicklung und Zusammenarbeit, Nr.8/9, September 2002, S. 244-245

Hungerford, Thomas/Solon, Gary (1987): Sheepskin Effects in the Returns to Education, in: The Review of Economics and Statistics, Vol. 69, Issue 1, Feb. 1987, S. 175-177.

IDB (1998): Facing up to Inequality in Latin America, Economic and Social Progress in Latin America, 1998-1999 Report, Washington D.C. 1998.

Jacoby, Hanan G. (1994): Borrowing Constraints and Progress Through School: Evidence from Peru, in: Review of Economics and Statistics, Vol. 76 , No. 1,1994 , S. 151-160. 
Jamison, Dean T./Lockheed, Marlaine E. (1987): Partizipation in Schooling: Determinants and Learning Outcomes in Nepal, in: Economic Development and Cultural Change, Vol. 35, No. 2, January 1987, S. 279-306.

KAS-Kurzinfo, Peru, April 1997

Katona, George (1980): How Expectations are Really Formed, in: Challenge, Vol. 23, No. 5, 1980, S. 32-39.

Kendrick, John W. (Hrsg.)(1984): International Comparisons of Productivity and Causes of the Slowdown, Cambridge, Massachusetts 1984.

King, Timothy (1980): Education and Income, World Bank Staff Working Paper 402, Washington D.C., 1980.

Kjellström, Christian (1999): Essays in Investment in Human Capital, Swedish Institute for Social Research, No.36, Edsbruk 1999.

Knight, J.B./Sabot, R.H./Hovey, D.C. (1992): Is the Rate of Return on Primary Schooling Really 26 Per Cent?; in: Journal of African Economies, Vol. 1, No. 2, S. 192-205.

Kodde, David A./Ritzen, Josef M.M. (1985): The Demand for Education under Capital Market Imperfections, in: European Economic Review, 28, S. 347362, North Holland 1985.

Lavy, Victor (1996): School Supply Constraints and Children's Educational Outcomes in Rural Ghana, Journal of Development Economics, Vol. 51, 1996, S. 291-314.

Lazear, Edward (1980): Family Background and Optimal Schooling Decisions, in: Review of Economics and Statistics, Vol. LXII, Feb. 1980, No. 1, S. 4251.

Leibivich, José/ Magnac, Thierry (1996): Demanda de Educación en las Zonas Rurales Colombianas, in: Coyuntura Económica, Vol. XXVI, No. 2, Junio de 1996.

Levison, Deborah/ Moe, Karine S. (1998): Household Work as a Deterrent to Schooling: An Analysis of Adolescent Girls in Peru, in: The Journal of Developing Areas, 32, Spring 1998, S. 339-356.

Lillard, Lee A./King, Elizabeth M. (1984): Methods for Analyzing School Choice with Household Survey Data, A Rand Note, July 1984, N-1963-AID. 
Lipton, Michael (1985): Education and Farm Efficiency: Comment, in: Economic Development and Cultural Change, Vol. 34, No.1, 1985, S. 167-168.

Lockheed, Marlaine E./Jamison, Dean T./Lau, Laurence J. (1980): Farmer Education and Farm Efficiency: A Survey, in: King, Timothy (Hrsg.): Education and Income, World Bank Staff Working Paper 402, Washington D.C., 1980, S. $111-152$.

Lockhead, Marlaine/Verspoor, Adriaan M. (1991): Improving Primary Education in Developing Countries, Oxford u.a. 1991.

Maddala, G.S. (1992): Introduction to Econometrics, 2nd edition, 1992.

Manski, Charles F. (1993): Adolescent Econometricians: How Do Youth Infer the Returns to Schooling?, in: Clotfelter, Charles T./Rothschild, Michael (Hrsg.): Studies of Supply and Demand in Higher Education, London, Chicago 1993, S. 43-57.

Mauro, Paolo/Spilimbergo, Antonio (1999): How do the Skilled and the Unskilled Respond to External Shocks?, in: IMF Staff Papers, Vol. 46, No. 1, March 1999, S. 1-17.

McCallum, Bennett T. (1980): The Significance of Rational Expectation Theory, in: Challenge, Vol. 22, No. 6, 1980, S. 37-41.

McMahon, Walter W. (1984): Why Families Invest in Education, in: Sudman, Seymour/Spaeth, Mary A. (eds.): The Collection and Analysis of Economic and Consumer Behavior Data: In Memory of Robert Ferber, Illinois 1984.

McMahon, W.W. (1987a): Consumption and Other Benefits of Education, in: Psacharopoulos, G. (Hrsg.): Economics of Education - Research and Studies, Oxford u.a. 1987, S.129-133.

McMahon, W.W. (1987b): Externalities in Education, in: Psacharopoulos, G. (Hrsg.): Economics of Education - Research and Studies, Oxford u.a. 1987, S. $133-137$.

McMahon, W.W. (1987c): Expected Rates of Return to Education, in: Psacharopoulos, G. (Hrsg.): Economics of Education - Research and Studies, Oxford u.a., 1987, S.187-196. 
McMahon, Walter M.Magner, Alan P. (1981): Expected Returns to Investment in Higher Education, in: The Journal of Human Resources, Vol. 16, No. 2, 1981, S. $274-285$.

Merrett, Stephen (1966): The Rate of Return to Education: A Critique, in: Oxford Economic Papers, Vol. 18, 1966, S. 289-303.

Mincer, Jacob (1974): Schooling, Experience, and Earnings, New York, 1974.

Ministerio de Educación (1995): Censo Escolar 1993, Lima, Diciembre 1995.

Ministerio de Educación (1997): Nueva Estructura del Sistema Educativo Peruano, Fundamentos de la Propuesta, Lima 1997.

Moock, Peter E. (1985): Education and Farm Efficiency: Reply, in: Economic Development and Cultural Change, Vol. 34, No.1, 1985, S. 169-172.

Moock, Peter E. (1981): Education and Technical Efficiency in Small-Farm Production, in: Economic Development and Cultural Change, Vol. 29, No. 4, 1981, S. 723-739.

Moock, Peter/Musgrove, Philip/Stelcner, Morton (1990): Education and Earnings in Perus Informal Nonfarm Family Enterprises, LSMS Working Paper, No. 64, Washington, D.C. 1990.

Moonwon Kang, Johan (1993): Why the rate of return to Education is Apparently Higher in LDCs than in Developed Countries: A Note; in: Journal of economic Studies, Vol. 20, No. 6, S. 32-36.

Müller-Benedict, Volker (1999): Strukturelle Grenzen sozialer Mobilität, in: Kölner Zeitschrift für Soziologie und Sozialpsychologie, Jg. 51, Heft 2, S. 313-338.

Plante, Patricia R. (1987): The Art of Decision Making: Issues and Cases in Higher Education, S. IX, New York, London 1987.

Psacharopoulos, George (1973): Returns to Education - An International Comparison, Amsterdam u.a. 1973.

Psacharopoulos, George (1984): The Contribution of Education to Economic Growth, in: Kendrick, John W. (Hrsg.): International Comparisons of Productivity and Causes of the Slowdown, Cambridge, Massachusetts 1984, S. 335-355. 
Psacharopoulos, George (1985): Returns to Education: A Further International Update and Implications, in: Journal of Human Resources, Vol. 20, No. 4, S. 583-597.

Psacharopoulos, George (Hrsg.) (1987): Economics of Education - Research and Studies, Oxford u.a. 1987.

Psacharopoulos, George (1994): Returns to Investment in Education: A Global Update; in: World Development, Vol. 22, No. 9, S. 1325-1343.

Psacharopoulos, George (1996): Economics of Education: A Research Agenda; in: Economics of Education Review, Vol. 15, No. 4, S. 339-344.

Psacharopoulos, George/Arriagada, Ana-María (1989): The Determinants of Early Age Human Capital Formation: Evidence from Brazil, in: Economic Development and Cultural Change, 37(4): 683-708, 1989.

Psacharopoulos, George/Woodhall, Maureen (1985): Education for Development - An Analysis of Investment Choices, Oxford 1985.

Rosenzweig, Mark R. (1990): Population Growth and Human Capital Investments: Theory and Evidence, in: Journal of Political Economy, Vol. 98, Part 2, No. 5, October 1990, S. S38-S70.

Schiefelbein, Ernesto (1992): Redefining Basic Education for Latin America: Lessons to be Learned from the Colombian Escuela Nueva, Paris 1992.

Schultz, Theodore W. (1961): Investment in Human Capital; in: American Economic Review, Vol. 51, 1961, S. 1-17.

Schultz, Theodore (1963): The Economic Value of Education, 1963.

Siphambe, Happy Kufigwa (2000): Rates of Return to Education in Botswana; in: Economics of Education Review, Vol. 19, S. 291-300.

Skyt Nielsen, Helena (1998): Low Demand for Primary Education: Tradition or Economic Incentives?, Centre for Labour Market and Social Research, Working Paper 98-05, March 1998.

Stelcner, Morton/Arriagada, Ana-Maria/Moock, Peter (1987): Wage Determinants and School Attainment among Man in Peru, LSMS Working Paper, No. 38, Washington, D.C. 1987. 
Stieglitz, Joseph E. (1975): The Theorie of "Screening", Education, and the Distribution of Income, in: The American Economic Review, Vol. 65, No. 3, June 1975 , S. 283-300.

Sudman, Seymour/Spaeth, Mary A. (Hrsg.) (1984): The Collection and Analysis of Economic and Consumer Behavior Data: In Memory of Robert Ferber, Illinois 1984.

Taubman, Paul (1989): Role of Parental Income in Educational Attainment, in: American Economic Review, Papers and Proceedings, Vol. 79, No. 2, May 1989, S. 57-61.

Tilak, Jandhyala B.G. (1993): Education and its Relation to Economic Growth, Poverty, and Income Distribution, World Bank Discussion Paper, No.46, Washington D.C., 1993.

Timmermann, Vincenz (1994): Bildung und Ausbildung als Determinanten der wirtschaftlichen Entwicklung, Beiträge zu Wirtschaftsforschung des Sozialökonomischen Seminars der Universität Hamburg, Nr. 78, 1994.

Van der Meer, Peter/Wielers, Rudi (1996): Educational Credentials and Trust in the Labour Market, in: Kyklos, Vol. 49, 1996, No. 1, S. 29-46.

Weiler, William C. (1989): A Flexible Approach to Modelling Enrollment Choice Behavior, in: Economics of Education Review, Vol. 8, No. 3, 1989, S. 277283.

Weißhuhn, Gernot (1977): Sozioökonomische Analyse von Bildungs- und Ausbildungsaktivitäten, Volkswirtschaftliche Schriften, Heft 259, Berlin 1977.

Weiss, Andrew (1995): Human Capital vs. Signalling Explanation of Wages, in: Journal of Economic Perspectives, Vol. 9, No. 4, 1995, S. 133-154.

Weltbank (1989): Weltenwicklungsbericht 1989, Washington, D.C. 1989.

Weltbank (2001): Peruvian Education at a Crossroad - Challenges and Opportunities for the $21^{\text {st }}$ Century, A World Bank Country Study, Washington, D.C. 2001.

Whitefield, Keith/Wilson R.A. (1991): Staying on in Full-time Education: The Educational Participation Rate of 16-year-olds, in: Economica, Vol. 58, No. 231, 1991, S. 391-404. 
Wieczorek-Zeul, Heidemarie (2002): Mit politischem Nachdruck der G8 Grundschulbildung für alle Kinder bis 2015, in: DSE (Hrsg.):E +Z - Entwicklung und Zusammenarbeit, Nr.8/9, September 2002, S. 236-239.

Winkler D.R. (1987): Screening Models and Education, in: Psacharopoulos, G.: Economics of Education - Research and Studies, Oxford u. a. 1987, S. 287-291.

Wolff, Laurence/Schiefelbein, Ernesto/Valenzuela, Jorge (1994): Improving the Quality of Primary Education in Latin America and the Caribbean, World Bank Discussion Papers 257, Washington, D.C. 1994.

Woodhall, Maureen. (1987): Human Capital Concepts, in: Psacharopoulos, G.: Economics of Education - Research and Studies, Oxford u. a., S. 21-24.

Wright, Robert E. (1999): The Rate of Return to private Schooling, IZA Discussion Paper Nr. 92, December 1999.

Zucchini/Sattler (2000): Lineare Modelle Teil 1: Varianzanalyse, Vorlesungsbegleitendes Skript, Göttingen 2000. 



\section{Anhang}

\section{Schichtkriterien}

\section{Tabelle A 1: Dominierende Merkmale der Schichten}

\begin{tabular}{|c|c|c|c|c|c|}
\hline \multirow{2}{*}{\multicolumn{2}{|c|}{ Charakteristika }} & \multicolumn{4}{|c|}{ SOZIOÖKONOMISCHE SCHICHTEN } \\
\hline & & $\operatorname{Hoch}(\mathrm{A})$ & Mittel (B) & Niedrig (C) & Sehr niedrig (D) \\
\hline 1 & - Geburtsort des Haushaltsvorstands & Lima & Lima & Sierra/Lima & Sierra/Lima \\
\hline 2 & - Rasse des Haushaltsvorstands & Weiß & Weiß & Mestize & Mestize \\
\hline 3 & - Bildung des Haushaltsvorstands & höhere & höhere & höhere & Primarschule/ \\
\hline 4 & - Schule der Kinder & $\begin{array}{l}\text { universitäre } \\
\text { privat }\end{array}$ & $\begin{array}{l}\text { universitäre } \\
\text { privat }\end{array}$ & $\begin{array}{l}\text { nicht universitäre } \\
\text { staatlich }\end{array}$ & $\begin{array}{l}\text { Sekundarschule } \\
\text { staatlich }\end{array}$ \\
\hline 5 & - Beruf des Haushaltsvorstands & $\begin{array}{l}\text { Unternehmer/ } \\
\text { Geschäftsführer }\end{array}$ & $\begin{array}{l}\text { Freiberufler/ } \\
\text { Angestellte }\end{array}$ & $\begin{array}{l}\text { kleine Händler/ } \\
\text { Techniker }\end{array}$ & $\begin{array}{l}\text { Arbeiter/ambulante } \\
\text { Händler }\end{array}$ \\
\hline 6 & $\begin{array}{l}\text { - Durchschnittliches monatliches } \\
\text { Einkommen }\end{array}$ & $\$ 2.500$ & $\$ 1.300$ & $\$ 300$ & $\$-200$ \\
\hline 7 & - Anzahl Personen pro Schlafzimmer & 1 & 1,8 & 2,4 & 3,4 \\
\hline 8 & - Wohngebiet & exklusiv & mittel & einfach & marginal \\
\hline 10 & - Front der Wohnung & eingezäunt (mind. 14 Meter) & 10 Meter & 6 Meter & 4 Meter \\
\hline 11 & - Zustand der Wohnung & gut & gut bis mittel & mittel & schlecht \\
\hline
\end{tabular}

Cuánto S.A. 


\section{Ermittlung der geeigneten Stichprobe}

Tabelle A 2: Bevölkerungsverteilung: Lima Stadt

\begin{tabular}{|c|c|c|c|c|c|c|c|}
\hline \multirow{2}{*}{\multicolumn{2}{|c|}{ Distrikte }} & \multirow{2}{*}{$\begin{array}{l}\text { Armuts- } \\
\text { indize }\end{array}$} & \multirow{2}{*}{$\begin{array}{l}\text { gesamt } \\
(1.000)\end{array}$} & \multicolumn{4}{|c|}{ Schichten } \\
\hline & & & & A & B & C & $\mathrm{D}$ \\
\hline & & & & 289.92 & & & \\
\hline & TOTAL & & 6.656 & 0 & 1.084 .130 & 1.990 .530 & 3.290 .370 \\
\hline 1 & Puente Piedra & 18,81 & 112 & 0 & 0 & 22.400 & 89.600 \\
\hline 2 & Ventanilla & 18,52 & 111 & 0 & 0 & 41.070 & 69.930 \\
\hline 3 & Ate & 13,89 & 290 & 0 & 26.100 & 26.100 & 237.800 \\
\hline 4 & Carabayllo & 12,99 & 116 & 0 & 6.960 & 27.840 & 81.200 \\
\hline 5 & S.J. de Lurigancho & 12,88 & 635 & 0 & 0 & 120.650 & 514.350 \\
\hline 6 & Los Olivos & 11,87 & 248 & 0 & 34.720 & 76.880 & 136.400 \\
\hline 7 & Villa El Salvador & 11,69 & 277 & 0 & 0 & 0 & 277.000 \\
\hline 8 & El Augustino & 10,58 & 168 & 0 & 0 & 20.160 & 147.840 \\
\hline 9 & S.J. de Miraflores & 10,46 & 309 & 0 & 3.090 & 95.790 & 210.120 \\
\hline 10 & Chorrillos & 9,7 & 236 & 2.360 & 44.840 & 54.280 & 134.520 \\
\hline 11 & Villa M. d. Triunfo & 9,08 & 287 & 0 & 0 & 106.190 & 180.810 \\
\hline 12 & Santa Anita & 8,65 & 129 & 0 & 9.030 & 42.570 & 77.400 \\
\hline 13 & Comas & 7,78 & 440 & 0 & 4.400 & 132.000 & 303.600 \\
\hline 14 & Callao & 7,49 & 403 & 0 & 24.180 & 217.620 & 161.200 \\
\hline 15 & Independencia & 7,2 & 200 & 0 & 0 & 54.000 & 146.000 \\
\hline 16 & S. M. de Porres & 5,67 & 414 & 0 & 4.140 & 202.860 & 207.000 \\
\hline 17 & Rimac & 4,71 & 207 & 0 & 0 & 78.660 & 128.340 \\
\hline 18 & La Molina & 4,03 & 85 & 32.300 & 34.850 & 15.300 & 2.550 \\
\hline 19 & La Victoria & 3,97 & 247 & 0 & 74.100 & 111.150 & 61.750 \\
\hline 20 & Cercado & 3,84 & 371 & 0 & 118.720 & 185.500 & 66.780 \\
\hline 21 & C. de la Legua & 3,77 & 40 & 0 & 0 & 38.800 & 1.200 \\
\hline 22 & S. de Surco & 3,52 & 219 & 78.840 & 98.550 & 15.330 & 28.470 \\
\hline 23 & Surquillo & 3,47 & 96 & 0 & 52.800 & 38.400 & 4.800 \\
\hline 24 & Barranco & 3,05 & 44 & 1.320 & 14.080 & 23.760 & 4.840 \\
\hline 25 & San Miguel & 3 & 128 & 0 & 89.600 & 32.000 & 6.400 \\
\hline 26 & Brena & 2,75 & 98 & 0 & 7.840 & 88.200 & 1.960 \\
\hline 27 & San Luis & 2,7 & 53 & 0 & 27.030 & 20.140 & 5.830 \\
\hline 28 & La Perla & 2,39 & 64 & 0 & 40.320 & 23.680 & 0 \\
\hline 29 & Magdalena & 2,18 & 53 & 6.360 & 37.100 & 8.480 & 1.060 \\
\hline 30 & Lince & 1,98 & 69 & 4.830 & 36.570 & 27.600 & 0 \\
\hline 31 & Bellavista & 1,85 & 75 & 0 & 41.250 & 33.750 & 0 \\
\hline 32 & Pueblo Libre & 1,69 & 81 & 0 & 74.520 & 1.620 & 1.620 \\
\hline 33 & San Borja & 1,53 & 109 & 65.400 & 42.510 & 1.090 & 0 \\
\hline 34 & La Punta & 1,51 & 7 & 140 & 6.370 & 490 & 0 \\
\hline 35 & Miraflores & 1,39 & 95 & 34.200 & 56.050 & 4.750 & 0 \\
\hline 36 & Jesus María & 1,26 & 71 & 0 & 69.580 & 1.420 & 0 \\
\hline 37 & San Isidro & 1,22 & 69 & 64.170 & 4.830 & 0 & $v$ \\
\hline
\end{tabular}

Fuente: Perú en Números 1996, Cuánto S.A. 
Tabelle A 3: Bevölkerungsverteilung: Lima Stadt, \%

\begin{tabular}{|c|c|c|c|c|c|c|c|}
\hline & \multirow[t]{2}{*}{ Distrikte } & \multirow{2}{*}{$\begin{array}{l}\text { Armuts- } \\
\text { indize }\end{array}$} & \multirow{2}{*}{$\begin{array}{c}\text { gesamt } \\
(\%)\end{array}$} & \multicolumn{4}{|c|}{ Schichten } \\
\hline & & & & A & $\mathrm{B}$ & $\mathrm{C}$ & $\mathrm{D}$ \\
\hline & TOTAL & & 100,00 & 4,36 & 16,29 & 29,91 & 49,44 \\
\hline 1 & Puente Piedra & 18,81 & 1,68 & 0 & 0 & 20 & 80 \\
\hline 2 & Ventanilla & 18,52 & 1,67 & 0 & 0 & 37 & 63 \\
\hline 3 & Ate & 13,89 & 4,36 & 0 & 9 & 9 & 82 \\
\hline 4 & Carabayllo & 12,99 & 1,74 & 0 & 6 & 24 & 70 \\
\hline 5 & S.J. de Lurigancho & 12,88 & 9,54 & 0 & 0 & 19 & 81 \\
\hline 6 & Los Olivos & 11,87 & 3,73 & 0 & 14 & 31 & 55 \\
\hline 7 & Villa El Salvador & 11,69 & 4,16 & 0 & 0 & 0 & 100 \\
\hline 8 & El Augustino & 10,58 & 2,52 & 0 & 0 & 12 & 88 \\
\hline 9 & S.J. de Miraflores & 10,46 & 4,64 & 0 & 1 & 31 & 68 \\
\hline 10 & Chorrillos & 9,7 & 3,55 & 1 & 19 & 23 & 57 \\
\hline 11 & Villa M. del Triunfo & 9,08 & 4,31 & 0 & 0 & 37 & 63 \\
\hline 12 & Santa Anita & 8,65 & 1,94 & 0 & 7 & 33 & 60 \\
\hline 13 & Comas & 7,78 & 6,61 & 0 & 1 & 30 & 69 \\
\hline 14 & Callao & 7,49 & 6,05 & 0 & 6 & 54 & 40 \\
\hline 15 & Independencia & 7,2 & 3,00 & 0 & 0 & 27 & 73 \\
\hline 16 & S. M. de Porres & 5,67 & 6,22 & 0 & 1 & 49 & 50 \\
\hline 17 & Rimac & 4,71 & 3,11 & 0 & 0 & 38 & 62 \\
\hline 18 & La Molina & 4,03 & 1,28 & 38 & 41 & 18 & 3 \\
\hline 19 & La Victoria & 3,97 & 3,71 & 0 & 30 & 45 & 25 \\
\hline 20 & Cercado & 3,84 & 5,57 & 0 & 32 & 50 & 18 \\
\hline 21 & C. de la Legua & 3,77 & 0,60 & 0 & 0 & 97 & 3 \\
\hline 22 & Santiago de Surco & 3,52 & 3,29 & 36 & 45 & 7 & 13 \\
\hline 23 & Surquillo & 3,47 & 1,44 & 0 & 55 & 40 & 5 \\
\hline 24 & Barranco & 3,05 & 0,66 & 3 & 32 & 54 & 11 \\
\hline 25 & San Miguel & 3 & 1,92 & 0 & 70 & 25 & 5 \\
\hline 26 & Brena & 2,75 & 1,47 & 0 & 8 & 90 & 2 \\
\hline 27 & San Luis & 2,7 & 0,80 & 0 & 51 & 38 & 11 \\
\hline 28 & La Perla & 2,39 & 0,96 & 0 & 63 & 37 & 0 \\
\hline 29 & Magdalena & 2,18 & 0,80 & 12 & 70 & 16 & 2 \\
\hline 30 & Lince & 1,98 & 1,04 & 7 & 53 & 40 & 0 \\
\hline 31 & Bellavista & 1,85 & 1,13 & 0 & 55 & 45 & 0 \\
\hline 32 & Pueblo Libre & 1,69 & 1,22 & 0 & 92 & 2 & 2 \\
\hline 33 & San Borja & 1,53 & 1,64 & 60 & 39 & 1 & 0 \\
\hline 34 & La Punta & 1,51 & 0,11 & 2 & 91 & 7 & 0 \\
\hline 35 & Miraflores & 1,39 & 1,43 & 36 & 59 & 5 & 0 \\
\hline 36 & Jesus María & 1,26 & 1,07 & 0 & 98 & 2 & 0 \\
\hline 37 & San Isidro & 1,22 & 1,04 & 93 & 7 & 0 & 0 \\
\hline
\end{tabular}

Fuente: Perú en Números 1996, Cuánto S.A. 
Tabelle A 4: Ermittlung der Stichprobe I

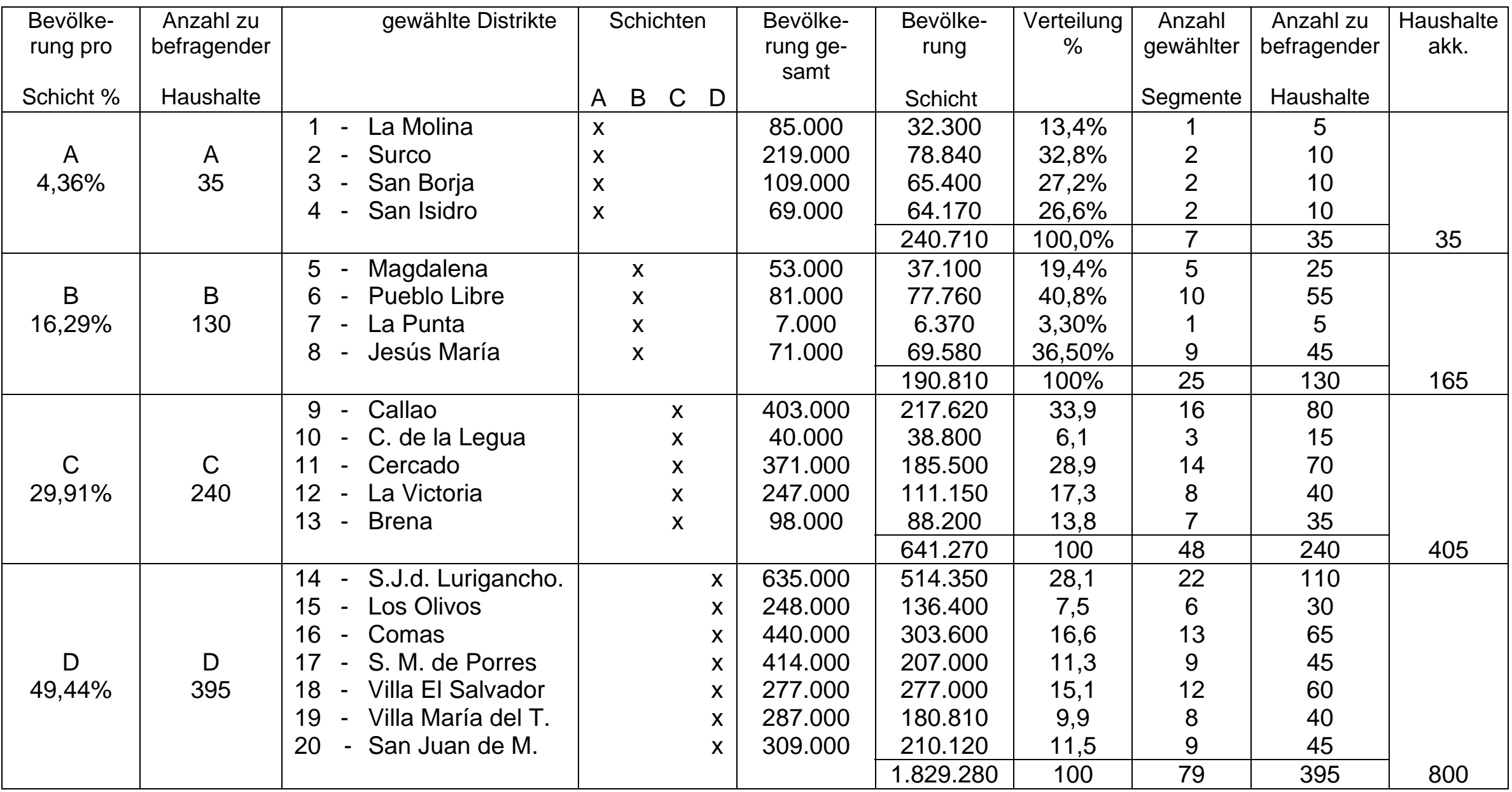


Tabelle A 5: Ermittlung der Stichprobe II

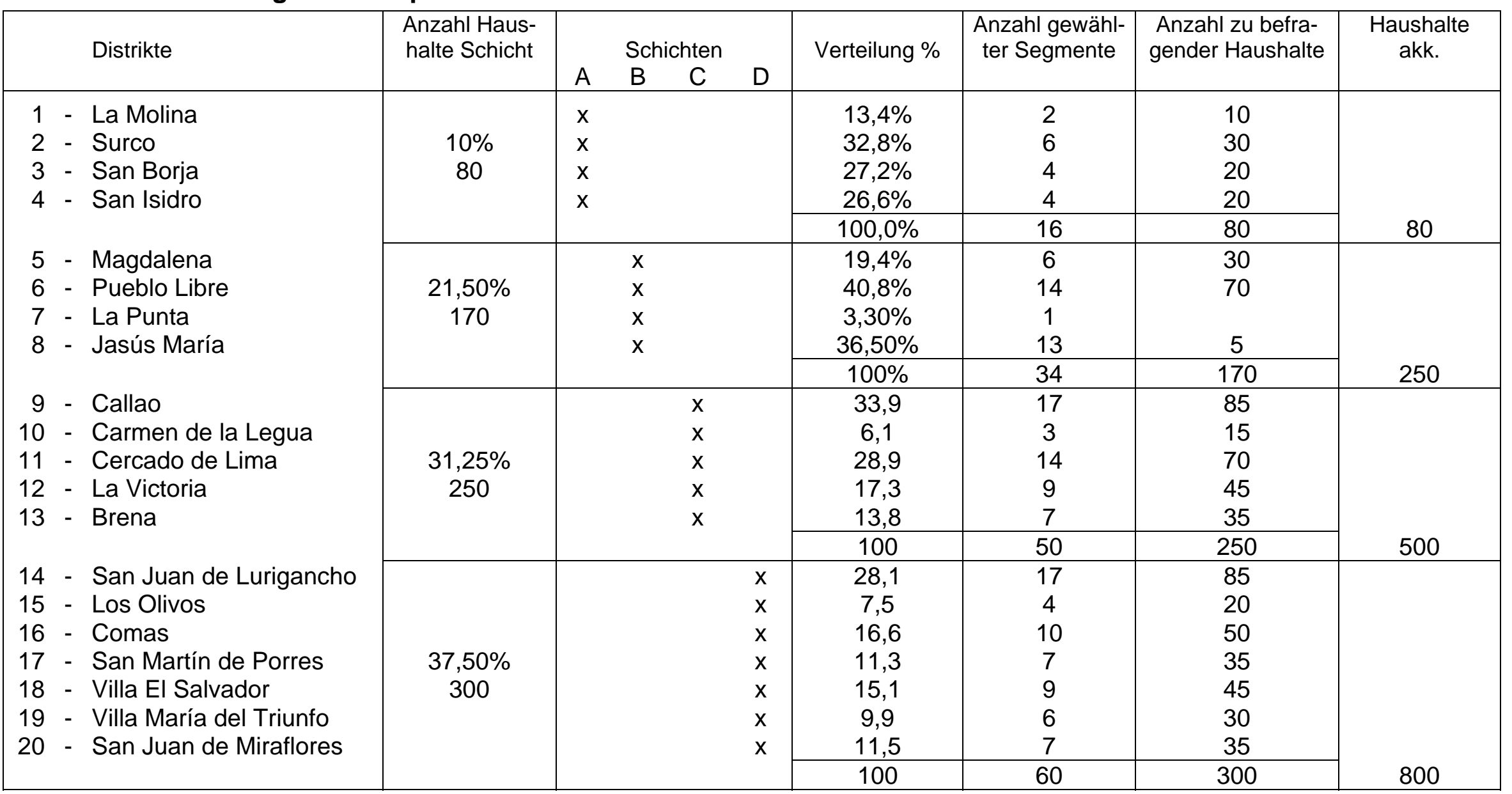




\section{Ermittlung der Expansionsfaktoren}

Tabelle A 6: Lima: Verteilung der Bevölkerung auf Haushalte, 1994

\begin{tabular}{lrrrr} 
& $\begin{array}{c}\text { Haushalte } \\
(1.000)\end{array}$ & $\begin{array}{c}\text { Haushalte } \\
(\%)\end{array}$ & $\begin{array}{c}\text { Bevölkerung } \\
(1.000)\end{array}$ & $\begin{array}{c}\text { Bevölkerung } \\
(\%)\end{array}$ \\
\hline TOTAL & $\mathbf{1 . 3 4 2 , 9 0}$ & $\mathbf{1 0 0 , 0 0 \%}$ & $\mathbf{6 . 4 5 5 , 8 0}$ & $\mathbf{1 0 0 , 0 0 \%}$ \\
& & & & \\
Hoch (A) & 56,4 & $4,20 \%$ & 242,8 & $3,76 \%$ \\
Mittel (B) & 259,2 & $19,30 \%$ & $1.122,2$ & $17,38 \%$ \\
Niedrig (C) & 491,5 & $36,60 \%$ & $2.362,5$ & $36,59 \%$ \\
Sehr niedrig (D) & 535,8 & $39,90 \%$ & $2.728,3$ & $42,26 \%$
\end{tabular}

Tabelle A 7: Lima: Haushalte nach Kindern im schulpflichtigen Alter, 1997

\begin{tabular}{lrrrrr} 
& gesamt & \multicolumn{5}{c}{ Schichten } \\
\cline { 3 - 6 } & & \multicolumn{1}{c}{$\mathrm{A}$} & $\mathrm{B}$ & $\mathrm{C}$ & $\mathrm{D}$ \\
\hline & & & & & \\
TOTAL & $\mathbf{1 . 4 6 3 . 0 2 7}$ & $\mathbf{6 1 . 4 4 7}$ & $\mathbf{2 8 2 . 3 6 4}$ & $\mathbf{5 3 5 . 4 6 8}$ & $\mathbf{5 8 3 . 7 4 8}$ \\
keins & 517.721 & 36.868 & 141.182 & 182.059 & 157.612 \\
& & & & & \\
SUB-TOTAL & $\mathbf{9 4 5 . 3 0 6}$ & $\mathbf{2 4 . 5 7 9}$ & $\mathbf{1 4 1 . 1 8 2}$ & $\mathbf{3 5 3 . 4 0 9}$ & $\mathbf{4 2 6 . 1 3 6}$ \\
eins & 353.526 & 14.133 & 64.944 & 128.512 & 145.937 \\
zwei & 333.526 & 9.217 & 50.826 & 139.222 & 134.262 \\
drei & 155.651 & 614 & 19.765 & 53.547 & 81.725 \\
vier & 76.297 & 0 & 2.824 & 26.773 & 46.700 \\
mind. fünf & 26.305 & 614 & 2.824 & 5.355 & 17.512 \\
& & & & &
\end{tabular}

Tabelle A 8: Lima: Expansionsfaktoren, 1997

Stichprobe Haushalte Expansionsfaktoren

\begin{tabular}{lrrr} 
Haushalte, gesamt & $\mathbf{8 0 1}$ & $\mathbf{1 . 4 6 3 . 0 2 7}$ & \\
Hoch (A) & 82 & 61.447 & 749,355 \\
Mittel (B) & 171 & 282.364 & $1.651,253$ \\
Niedrig (C) & 250 & 535.468 & $2.141,87$ \\
Sehr niedrig (D) & 298 & 583.748 & $1.958,89$ \\
\multicolumn{4}{c}{} \\
Haushalte mit Kindern im schulfähigen Alter \\
Hoch (A) \\
Mittel (B) & $\mathbf{8 0 1}$ & $\mathbf{9 4 5 . 3 0 5 , 6 8}$ & \\
Niedrig (C) & 82 & 24.579 & 299,742 \\
Sehr niedrig (D) & 171 & 141.182 & 825,626 \\
& 250 & 353.409 & $1.413,64$ \\
\end{tabular}

Cuánto, S.A. 


\section{Ergebnisse der Varianzanalyse}

Die Varianzanalyse ist ein Analyseverfahren zur Durchführung von Mittelwertvergleichen zwischen mehreren Gruppen. Die Grundidee besteht darin, die gesamte Varianz des zu erklärenden Merkmals (der abhängigen Variablen) aufzuteilen in die Varianz zwischen den Gruppen und die Varianz innerhalb der Gruppen. Sind die Unterschiede zwischen den Gruppen relativ groß bei gleichzeitig nicht allzu großer Varianz innerhalb der Gruppen, so kann man davon ausgehen, dass die Gruppenzugehörigkeit einen Einfluss auf die abhängige Variable hat. Formal wird dies geprüft über den F-Test.

$\mathrm{F}=$ Prüfgröße $=$ Varianz zwischen den Gruppen/Varianz innerhalb der Gruppen

$\mathrm{P}=$ Irrtumswahrscheinlichkeit= alpha

Tabelle A 9: Varianzanalyse, Ertragserwartungen allgemein

\begin{tabular}{|c|c|c|c|c|c|}
\hline $\begin{array}{l}\text { Messvariable: } \\
\text { gruppiert nach: }\end{array}$ & $\begin{array}{l}\text { Einkommenserwa } \\
\text { Schichten } \\
\text { Quadratsumme }\end{array}$ & $\begin{array}{l}\text { tung ohne Bildung } \\
\text { Freiheitsgrade }\end{array}$ & $\begin{array}{l}\text { allgemein } \\
\text { mittlere QS }\end{array}$ & $F$ & $\mathbf{P}$ \\
\hline \multirow{3}{*}{$\begin{array}{l}\text { Zwischen } \\
\text { Innerhalb } \\
\text { Gesamt }\end{array}$} & $279.550,28$ & 3 & $93.183,42$ & 3,64983 & 0,012383 \\
\hline & $20.348 .099,98$ & 797 & $25.530,86$ & & \\
\hline & $20.627 .650,26$ & 800 & $25.784,56$ & & \\
\hline $\begin{array}{l}\text { Messvariable: } \\
\text { gruppiert nach: }\end{array}$ & \multicolumn{5}{|c|}{$\begin{array}{l}\text { Einkommenserwartung mit Primaria, allgemein } \\
\text { Schichten }\end{array}$} \\
\hline \multirow{3}{*}{$\begin{array}{l}\text { Zwischen } \\
\text { Innerhalb } \\
\text { Gesamt }\end{array}$} & $759.051,78$ & 3 & $253.017,26$ & 5,11056 & 0,001657 \\
\hline & $39.458 .414,40$ & 797 & $49.508,67$ & & \\
\hline & $40.217 .466,18$ & 800 & $50.271,83$ & & \\
\hline \multirow[t]{2}{*}{$\begin{array}{l}\text { Messvariable: } \\
\text { gruppiert nach: }\end{array}$} & \multicolumn{5}{|c|}{$\begin{array}{l}\text { Einkommenserwartung mit Sekundaria, allgemein } \\
\text { Schichten }\end{array}$} \\
\hline & Quadratsumme & Freiheitsgrade & mittlere QS & $\mathbf{F}$ & $\mathbf{P}$ \\
\hline \multirow{3}{*}{$\begin{array}{l}\text { Zwischen } \\
\text { Innerhalb } \\
\text { Gesamt }\end{array}$} & $\begin{array}{r}5.739 .089,79 \\
\end{array}$ & 3 & $1.913 .029,93$ & 12,6475 & $4,4 \mathrm{E}-08$ \\
\hline & $120.552 .112,12$ & 797 & $151.257,35$ & & \\
\hline & $126.291 .201,91$ & 800 & $157.864,00$ & & \\
\hline \multirow[t]{2}{*}{$\begin{array}{l}\text { Messvariable: } \\
\text { gruppiert nach: }\end{array}$} & \multicolumn{5}{|c|}{$\begin{array}{l}\text { Einkommenserwartung mit höherer Bildung allgemein } \\
\text { Schichten }\end{array}$} \\
\hline & Quadratsumme & Freiheitsgrade & mittlere QS & $\mathbf{F}$ & $\mathbf{P}$ \\
\hline \multirow{3}{*}{$\begin{array}{l}\text { Zwischen } \\
\text { Innerhalb } \\
\text { Gesamt }\end{array}$} & $280.523 .752,27$ & 3 & $93.507 .917,42$ & 56,863 & $2,58 \mathrm{E}-33$ \\
\hline & \begin{tabular}{|l|}
$1.310 .619 .789,74$ \\
\end{tabular} & 797 & $1.644 .441,39$ & & \\
\hline & \begin{tabular}{|l|}
$1.591 .143 .541,01$ \\
\end{tabular} & 800 & $1.988 .929,42$ & & \\
\hline
\end{tabular}


Tabelle A 10: Varianzanalyse, Ertragserwartungen für die eigenen Kinder

\begin{tabular}{|c|c|c|c|c|c|}
\hline \multicolumn{6}{|c|}{ Ertragserwartungen für die Primaria } \\
\hline \multirow[t]{2}{*}{$\begin{array}{l}\text { Messvariable: } \\
\text { gruppiert nach: }\end{array}$} & \multicolumn{5}{|c|}{$\begin{array}{l}\text { Einkommenserwartung für die eigenen Kinder, Primaria } \\
\text { Schichten }\end{array}$} \\
\hline & Quadratsumme & Freiheitsgrade & mittlere QS & $\mathbf{F}$ & $\mathbf{P}$ \\
\hline \multirow{3}{*}{$\begin{array}{l}\text { Zwischen } \\
\text { Innerhalb } \\
\text { Gesamt }\end{array}$} & $1.223 .048,05$ & 3 & $407.682,68$ & 9,8266 & $2,28 \mathrm{E}-06$ \\
\hline & $33.065 .673,92$ & 797 & $41.487,67$ & & \\
\hline & $34.288 .721,97$ & 800 & $42.860,90$ & & \\
\hline \multirow[t]{2}{*}{$\begin{array}{l}\text { Messvariable: } \\
\text { gruppiert nach: }\end{array}$} & $\begin{array}{l}\text { Rentabilitätseinko } \\
\text { Schichten }\end{array}$ & רmen Primaria & & & \\
\hline & Quadratsumme & Freiheitsgrade & mittlere QS & $\mathbf{F}$ & $\mathbf{P}$ \\
\hline \multirow{3}{*}{$\begin{array}{l}\text { Zwischen } \\
\text { Innerhalb } \\
\text { Gesamt }\end{array}$} & $19.826 .258,63$ & 3 & $6.608 .752,88$ & 20,7017 & $6,36 \mathrm{E}-13$ \\
\hline & $254.432 .034,76$ & 797 & $319.237,18$ & & \\
\hline & $274.258 .293,40$ & 800 & $342.822,87$ & & \\
\hline \multicolumn{6}{|c|}{ Ertragserwartungen für die Sekundaria } \\
\hline \multirow[t]{2}{*}{$\begin{array}{l}\text { Messvariable: } \\
\text { gruppiert nach: }\end{array}$} & \multicolumn{5}{|c|}{$\begin{array}{l}\text { Einkommenserwartung für die eigenen Kinder, Sekundaria } \\
\text { Schichten }\end{array}$} \\
\hline & Quadratsumme & Freiheitsgrade & mittlere QS & $\mathbf{F}$ & $\mathbf{P}$ \\
\hline \multirow{3}{*}{$\begin{array}{l}\text { Zwischen } \\
\text { Innerhalb } \\
\text { Gesamt }\end{array}$} & $11.364 .192,53$ & 3 & $3.788 .064,18$ & 15,1357 & 1,38E-09 \\
\hline & $199.467 .559,72$ & 797 & $250.272,97$ & & \\
\hline & $210.831 .752,25$ & 800 & $263.539,69$ & & \\
\hline \multirow[t]{3}{*}{$\begin{array}{l}\text { Messvariable: } \\
\text { gruppiert nach: }\end{array}$} & \multicolumn{5}{|c|}{$\begin{array}{l}\text { Rentabilitätseinkommen Sekundaria } \\
\text { Schichten }\end{array}$} \\
\hline & Quadratsumme & Freiheitsgrade & mittlere QS & $\mathbf{F}$ & $\mathbf{P}$ \\
\hline & & & $14.142 .775,7$ & & \\
\hline \multirow{3}{*}{$\begin{array}{l}\text { Zwischen } \\
\text { Innerhalb } \\
\text { Gesamt }\end{array}$} & $42.428 .327,32$ & 3 & 7 & 27,8821 & $3,76 \mathrm{E}-17$ \\
\hline & $404.266 .934,78$ & 797 & $507.235,80$ & & \\
\hline & $446.695 .262,10$ & 800 & $558.369,08$ & & \\
\hline
\end{tabular}

